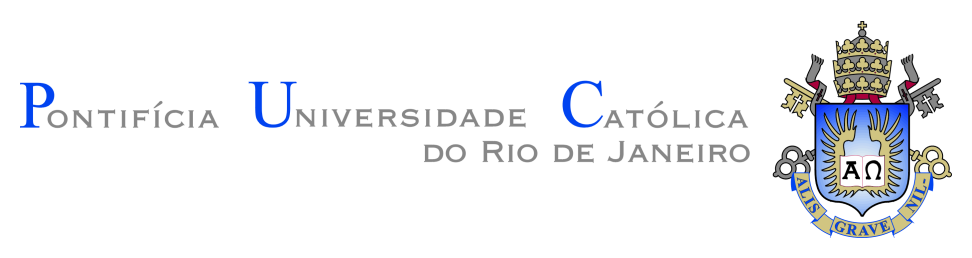

Gabrielle Saller Nornberg

\title{
Methods of the Regularity Theory in the Study of Partial Differential Equations with Natural Growth in the Gradient
}

Tese de Doutorado

Thesis presented to the Programa de Pós-graduação em Matemática of PUC-Rio in partial fulfillment of the requirements for the degree of Doutor em Matemática.

Advisor: Prof. Boyan Slavchev Sirakov 


\section{Pontifícia Universidade Catálica $_{\text {a }}$

\section{Gabrielle Saller Nornberg}

Methods of the Regularity Theory in the Study of Partial Differential Equations with Natural Growth in the Gradient

Thesis presented to the Programa de Pós-graduação em Matemática of PUC-Rio in partial fulfillment of the requirements for the degree of Doutor em Matemática. Approved by the undersigned Examination Committee.

Prof. Boyan Slavchev Sirakov

Advisor

Departamento de Matemática - PUC-Rio

Prof. Alexander Quaas Berger

Departamento de Matemática - Universidad Técnica Federico Santa María

Prof. Carlos Tomei

Departamento de Matemática - PUC-Rio

Prof. Diego Ribeiro Moreira

Departamento de Matemática - UFC

Prof. Ederson Moreira dos Santos Departamento de Matemática ICMC - USP

Prof. João Marcos Bezerra do Ó Departamento de Matemática - UFPB

Prof. Julio Daniel Rossi

Departamento de Matemática - Universidad de Buenos Aires

Prof. Liliane de Almeida Maia

Departamento de Matemática - UnB

Prof. Wladimir Augusto das Neves Instituto de Matemática - UFRJ

Prof. Márcio da Silveira Carvalho Vice Dean of Graduate Studies

Centro Técnico Científico - PUC-Rio

Rio de Janeiro, April the 26th, 2018 
All rights reserved.

\section{Gabrielle Saller Nornberg}

Bachelor's degree in Mathematics by Universidade Federal de Pelotas (UFPel) in 2012. Master degree in Mathematics by Instituto Nacional de Matemática Pura e Aplicada (IMPA) in 2014, with emphasis in Analysis. During the Ph.D. at PUC-Rio, also studied at Centre d'Analyse et de Mathématique Sociales (CAMS) of École des Hautes Études en Sciences Sociales (EHESS). Research interests include Regularity, Existence, Nonexistence and Multiplicity of Solutions of Partial Differential Equations.

Bibliographic data

Nornberg, Gabrielle Saller

Methods of the Regularity Theory in the Study of Partial Differential Equations with Natural Growth in the Gradient / Gabrielle Saller Nornberg; advisor: Boyan Slavchev Sirakov. - Rio de janeiro: PUC-Rio, Departamento de Matemática, 2018.

v., 136 f: il. color. ; $30 \mathrm{~cm}$

Tese (doutorado) - Pontifícia Universidade Católica do Rio de Janeiro, Departamento de Matemática.

Inclui bibliografia

1. Matemática - Teses. 2. Regularidade;. 3. Multiplicidade de soluções;. 4. Existência;. 5. Estimativas a priori;. 6. Viscosidade.. I. Sirakov, Boyan. II. Pontifícia Universidade Católica do Rio de Janeiro. Departamento de Matemática. III. Título. 
To my grandmother Ceni (in memoriam), with love. 


\section{Acknowledgments}

Thank you God for being my pillar of strength and for putting in my life such extraordinary people. First and foremost, Prof. Boyan Sirakov, great mathematician who, with his kindness, brings out the best in people; I am honored to learn from him everyday and I deeply admire him. Moreover, nothing would be possible if it were not for the guidance of Prof. Andrei and Lioudmila Bourchtein, eternal advisors, and of Prof. Emanuel Carneiro; I will always be indebted to them.

Several professors who encouraged me and gave me the support I needed to move on include Giovanni Nunes, Roberto Imbuzeiro and Carlos Tomei, representing UFPel, Impa and PUC-Rio in my academic journey. My sincere gratitude to the members of the jury for the attention and for the valuable discussions in the last days of the doctorate.

I warmly thank the pleasant environment of PUC-Rio's Department of Mathematics. The caring Mr. Orlando, Creuza, Kátia, Carlos and all team of professors. The fruitful discussions with my colleagues Dania, Tiago, Marcelo, Renan and the friendly atmosphere of our 850 office that also entails Cléa, Makson, Tamires, Letícia, Francisco, Fabrício, Renata, Allyson; André, David, Lilian, Viviana, etc. Some old friends as Jennifer, Adriana, Mateus, Diogo, Gabriel, Conrado, Caio, Alan, etc. since the time I was taking my masters.

Thanks to the friends I have made in Paris: Thomas, François, Beniada, Romain, Noemi, Fairouz, Charles, Samuel, Andrea and Alessandro; specially to the professors Henri Berestycki, Luca Rossi and Louis Jeanjean for receiving me so kindly in their institutes. Notable acknowledgement to Prof. Angela, muse of inspiration, and to the friends of the french literature class.

I am strongly grateful for the support given by my family; the incentive of my parents and, above all, my mother's perseverance to overcome the difficulties. I also thank the cousins Aline, Fernando, Diani, Tardelli and their children, our "carioca" family, as well as Heliete, Vera, Luiz, Paulo, Lorena, Henrique, Cintia, Tom, etc. for their friendship.

I cannot imagine myself in Rio all these years without my brother backing me up; Vagner Nornberg, whom I respect and I am proud of.

Mainly, I am thankful to my beloved husband, Felipe Germani, who gives me unconditional support in my decisions and is always by my side.

Last I acknowledge the financial support provided by PUC-Rio, Capes-Proex and Capes-Pdse grant 88881.134627/2016-01.

I wish one day my master colleagues and I can mitigate Leandro's loss, one of the greatest talents that our country has ever seen, a great person too. I hope that our theses can honor his memory. 


\section{Abstract}

Nornberg, Gabrielle Saller; Sirakov, Boyan (Advisor). Methods of the Regularity Theory in the Study of Partial Differential Equations with Natural Growth in the Gradient. Rio de Janeiro, 2018. 136p. Tese de doutorado - Departamento de Matemática, Pontifícia Universidade Católica do Rio de Janeiro.

In this Ph.D. thesis we study a class of uniformly elliptic partial differential equations of second order in fully nonlinear nondivergence form with superlinear growth in the gradient and measurable coefficients. For equations with quadratic growth, we prove that multiplicity of solutions occurs when the operator is not coercive. We investigate the qualitative behavior of the continuums of solutions obtained for a parameterized family of problems. For this, we extend the Caffarelli-Święch-Winter $C^{1, \alpha}$ regularity estimates to equations with at most quadratic gradient growth, showing that the solutions are continuously differentiable up to the boundary. Furthermore, we show a priori estimates in the uniform norm using purely nonlinear techniques in the nondivergence form, such as Harnack type inequalities and a Vázquez's strong maximum principle for equations of our type.

\section{Keywords}

Regularity; Multiplicity of solutions; Existence; A priori estimates; Viscosity. 


\section{Resumo}

Nornberg, Gabrielle Saller; Sirakov, Boyan. Métodos da Teoria de Regularidade no Estudo de Equações Diferenciais Parciais com Crescimento Natural no Gradiente. Rio de Janeiro, 2018. 136p. Tese de Doutorado - Departamento de Matemática, Pontifícia Universidade Católica do Rio de Janeiro.

Nesta tese de Doutorado estudamos uma classe de equações diferenciais parciais de segunda ordem, uniformemente elípticas, completamente não-lineares na forma não-divergência, com crescimento superlinear no gradiente e coeficientes mensuráveis. Para equações com crescimento quadrático, provamos que ocorre multiplicidade de soluções quando o operador não é coercivo e investigamos o comportamento qualitativo dos contínuos de soluções obtidos para uma família parametrizada de problemas. Para isso, estendemos a regularidade e as estimativas $C^{1, \alpha}$ de Caffarelli-Święch-Winter para equações com crescimento, no máximo quadrático, no gradiente, mostrando que as soluções são continuamente diferenciáveis até o bordo. Além disso, mostramos estimativas a priori na norma uniforme via técnicas puramente não-lineares na forma não-divergência, entre elas desigualdades do tipo Harnack e o princípio do máximo forte de Vázquez para equações de nosso tipo.

\section{Palavras-chave}

Regularidade; Multiplicidade de soluções; Existência; Estimativas a priori; Viscosidade. 


\section{Table of contents}

$\begin{array}{lll}1 & \text { Introduction } & 10\end{array}$

2 Preliminaries $\quad 15$

3 Hölder Regularity $\quad 31$

3.1 Main Regularity Results $\quad 32$

3.2 Proof of theorem 3.1. 36

3.2.1 Local Regularity 36

3.2.2 Boundary Regularity 47

3.2.3 The Global Blend $\quad 55$

$\begin{array}{lll}3.3 W^{2, p} \text { Results } & 60\end{array}$

4 The Eigenvalue Problem $\quad 63$

4.1 The Krein-Rutman Theorem $\quad 74$

5 A Priori Bounds and Multiplicity Results $\quad 76$

5.1 Main Results $\quad 77$

5.2 A Priori Bounds $\quad 82$

5.2.1 Proof of Lemma 5.8 90

6 The Degree Theory Approach $\quad 93$

6.1 Existence results through fixed point theorems 93

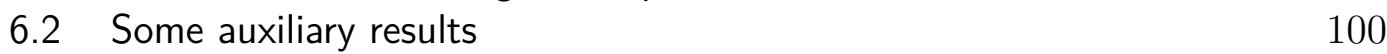

6.3 Proof of Theorem 5.3 105

6.4 Proof of Theorem 5.4 111

6.5 Proof of Theorem 5.5 114

$\begin{array}{ll}\text { Bibliography } & 119\end{array}$

$\begin{array}{ll}\text { A Notations } & 128\end{array}$

B Quadratic ABP 129

$\begin{array}{ll}\text { C A property of functions } & 131\end{array}$

D The Local Maximum Principle for $C$-viscosity solutions 133 


\section{List of figures}

Figure 3.1 Illustration of the variable change, from $B_{2 \sigma}^{+}(z)$ to $B_{2}^{\nu}(0) . \quad 51$

Figure 5.1 Illustration of theorem 5.4.

Figure 5.2 Illustration of theorem 5.5. 


\section{1 \\ Introduction}

This thesis is devoted to the study of regularity, existence and multiplicity of viscosity solutions for a class of fully nonlinear uniformly elliptic equations with quadratic growth in the gradient.

The notion of viscosity solution began to be developed within the differential equations universe in the 1980s, with the works of Crandall and Lions [1], [2]. Since then it has been an enormous influence on the study of fully nonlinear elliptic and parabolic PDEs. This is so because its definition weaker, where only continuity of solutions is required - is purely based upon the maximum principle, which is advantageous when we have an equation in nondivergence form. The first notion of viscosity solution, namely $C$-viscosity [3], had to be improved to cover the case when the coefficients or/and the right hand side of the equation are no longer continuous but merely measurable, which lead to the introduction of the $L^{p}$-viscosity notion [4]. In general, without the continuity hypothesis on the nonlinearity, the uniqueness is lost even for the standard Dirichlet problem for linear equations with bounded coefficients [5]. Therefore, we can expect multiplicity of solutions.

On the other hand, the study of quasilinear elliptic equations with quadratic dependence in the gradient also had its beginning in the ' $80 \mathrm{~s}$, essentially with the works of Boccardo, Murat and Puel [6], [7] and became a relevant research topic which still develops. This type of nonlinearity often appears in risk-sensitive stochastic and large deviation problems, hence their practical importance. Moreover, the set of equations under the form $L u=g(x, u, D u)$, where $L$ is a second order general operator and $g$ has quadratic growth in the gradient, is invariant with respect to smooth changes of the function $u$ and variable $x$, what makes this class also theoretically important. This fact was first observed by Kazdan and Kramer in [8] and it is responsible for this class to be usually referred as having natural growth in the gradient. 
To acquaint ourselves with the notation, we consider the following model equation which features a second order uniformly elliptic operator with quadratic growth in the gradient

$$
L u+c(x) u+\langle M(x) D u, D u\rangle=h(x)
$$

in a bounded $C^{1,1}$ domain $\Omega$ in $\mathbb{R}^{n}$ with a Dirichlet boundary condition $u=0$ on $\partial \Omega$. Here, $c, h \in L^{p}(\Omega), p>n \geq 1, c \not \equiv 0$ and the matrix $M$ satisfies the nondegeneracy condition $0<\mu_{1} I \leq M \leq \mu_{2} I$; with solutions of this Dirichlet problem understood as being continuous up to the boundary, so bounded. In [6], [7] the authors carried out a rather complete study of solvability of strictly coercive equations in divergence form, that is, when $L$ is the divergence of an expression of $x, u$, and $D u$. Strictly coercive for (ME) means that $c(x) \leq c_{0}<0$, and then uniqueness of solutions is to be expected, see [9], [10]. For weakly coercive equations (when $c \leq 0$ ), existence and uniqueness can be proved only under a smallness assumption on $c$ and $M$, as was first observed by Ferone and Murat [11]. All these pioneering works use the weak integral formulation of PDEs in divergence form.

In [12] Sirakov showed that the same type of existence and uniqueness results extends to general fully nonlinear coercive (i.e. proper) equations in nondivergence form, with superlinear growth in the gradient and unbounded coefficients, by using techniques based on the maximum principle. Moreover, $C^{\alpha}$ regularity results were developed there for fully nonlinear uniformly elliptic equations with at most quadratic growth in the gradient, in the most general setting of measurable coefficients for $L^{p}$-viscosity solutions.

In that paper it was also observed, for the first time and with a simple example with the Laplacian (specifically for $L=\Delta, c=1, M=I, h=0$ ), that the solution set may be very different in the nonproper case $c>0$, and in particular more than one solution may appear. This example leveraged the study of such equations over a new research strand, whose main objective is the qualitative analysis of the set of solutions. Subsequent results were established in [13], [14], [15], [16], [17], [18], still using energy methods.

This Ph.D. thesis can be seen as a natural continuation of the regularity, existence and multiplicity results initiated in [12]. In particular, our work attempts to contribute to the theory of nondivergence fully nonlinear equations with superlinear gradient growth and unbounded coefficients, in the setting of $L^{p}$-viscosity solutions. This thesis covers and develops the results of the following research articles 
[19] Nornberg, G.; Sirakov, B. A priori bounds and multiplicity for fully nonlinear equations with quadratic growth in the gradient. arXiv:1802.01661;

[20] Nornberg, G. S. $C^{1, \alpha}$ regularity for fully nonlinear elliptic equations with superlinear growth in the gradient. arXiv:1802.01643.

We stress that nondivergence (fully nonlinear) equations with natural growth are particularly relevant for applications, since problems with such dependence in the gradient are abundant in control and game theory [21], and more recently in mean-field problems [22], where Hamilton-Jacobi-Bellman and Isaacs operators appear as infinitesimal generators of the underlying stochastic processes.

The greatest challenge in these studies is to obtain regularity and a priori estimates for the solutions. We establish complete results of this nature for fully nonlinear uniformly elliptic equations in nondivergence form. In chapter 3 we compile the general $C^{1, \alpha}$ regularity and estimates for fully nonlinear equations with superlinear growth in the gradient and unbounded coefficients.

Our results are valid when $L$ in (ME) is a general Hamilton-JacobiBellman operator. However, to accommodate the reader and to elucidate the main ideas, let us give the statement of some of our results for $L^{p}$-viscosity solutions of $(\mathrm{ME})$, in the very particular case when $L$ is a second order uniformly elliptic linear operator in nondivergence form; namely

$$
L u=\operatorname{tr}\left(A(x) D^{2} u\right)+b(x) \cdot D u
$$

where $A$ is a matrix function which is continuous up to the boundary and whose eigenvalues belong to some finite and closed interval in $(0,+\infty)$ and $b$ is some bounded vector function. Consider $\Omega, c, h$ and $M$ under the assumptions above (actually, for the regularity results, $M$ can be any bounded matrix).

Theorem 1.1 ( $C^{1, \alpha}$ Regularity) Let $u$ be an $L^{p}$-viscosity solution of (ME), with $u \in C(\bar{\Omega})$ and $\|u\|_{L^{\infty}(\Omega)}+\|h\|_{L^{p}(\Omega)} \leq C_{0}$. Then there exists $\alpha \in(0,1)$ such that $u \in C^{1, \alpha}(\bar{\Omega})$ and satisfies the estimate

$$
\|u\|_{C^{1, \alpha}(\bar{\Omega})} \leq C\left\{\|u\|_{L^{\infty}(\Omega)}+\|h\|_{L^{p}(\Omega)}\right\}
$$

where $C$ and $\alpha$ depend only on the $L^{p}$-norm of the coefficients of the equation, on the ellipticity constants of $L$ and on $C_{0}$.

As an application, $W^{2, p}$ regularity results and a generalized Nagumo's lemma are established at the end of chapter 3. In particular, for the linear operator $L$, this regularity implies that (bounded) solutions of (ME) are strong, 
in the sense that they belong to the Sobolev space $W^{2, p}(\Omega)$ and satisfy the equation at almost every point.

In chapter 4 we present miscellaneous results on first eigenvalues of nonlinear operators, in the sense of weighted eigenvalues with unbounded coefficients - they play an important role in the proof of the multiplicity and nonexistence results.

We consider the following parameterized version of (ME)

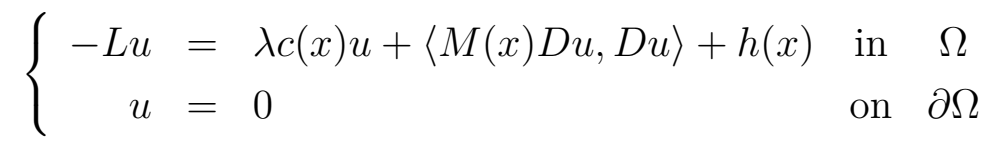

indexed by $\lambda \in \mathbb{R}$, with coefficients $c, h \in L^{\infty}(\Omega)$ and $c \supsetneqq 0$. For a priori estimates and multiplicity results related to $\left(P_{\lambda}\right)$, we also need to assume that the problem $\left(P_{0}\right)$ has a solution $u_{0}$.

In section 5.2 we prove that solutions of $\left(P_{\lambda}\right)$ are bounded in the uniform norm by a new method, as suggested in [12]. Upper bounds are based on some standard estimates from regularity theory, such as half-Harnack inequalities, and their recent boundary extensions in [23]. On the other hand, lower bounds are shown to be equivalent, somewhat surprisingly, to a Vázquez type strong maximum principle for our equations, which we also establish. Our methods are (necessarily) very different from those in the preceding works, since for us, even in the linear scenario, no integral formulation of the equation is available.

Theorem 1.2 (A priori uniform bounds) Let $\Lambda_{1}, \Lambda_{2}$ with $0<\Lambda_{1}<\Lambda_{2}$. Then every $L^{p}$-viscosity solution $u$ of $\left(P_{\lambda}\right)$ satisfies

$$
\left\|u^{-}\right\|_{L^{\infty}(\Omega)} \leq C, \text { for all } \lambda \in\left[0, \Lambda_{2}\right], \quad\left\|u^{+}\right\|_{L^{\infty}(\Omega)} \leq C, \text { for all } \lambda \in\left[\Lambda_{1}, \Lambda_{2}\right] \text {, }
$$

where $C$ depends on $n, p, \mu_{1}, \mu_{2}, \Omega, \Lambda_{1}, \Lambda_{2},\|b\|_{L^{\infty}(\Omega)},\|c\|_{L^{\infty}(\Omega)},\|h\|_{L^{\infty}(\Omega)}$, $\left\|u_{0}\right\|_{L^{\infty}(\Omega)}$, the ellipticity constants of $L$, the $C^{1,1}$ character of the boundary, and the set where $c>0$.

As a consequence, we obtain the following multiplicity result.

Theorem 1.3 (Multiplicity of solutions) For $\lambda \leq 0$, the problem $\left(P_{\lambda}\right)$ has a unique $L^{p}$-viscosity solution $u_{\lambda}$. Moreover, the set

$$
\Sigma=\left\{(\lambda, u) \in \mathbb{R} \times C^{1}(\bar{\Omega}) ;(\lambda, u) \text { solves }\left(P_{\lambda}\right)\right\}
$$

possesses an unbounded component $\mathcal{C}^{+} \subset[0,+\infty] \times C^{1}(\bar{\Omega})$ such that $\mathcal{C}^{+} \cap(\{0\} \times$ $\left.C^{1}(\bar{\Omega})\right)=\left\{u_{0}\right\}$ and satisfies 
(i) either it bifurcates from infinity to the right of the axis $\lambda=0$ with the corresponding solutions having a positive part blowing up to infinity in $C(\bar{\Omega})$ as $\lambda \rightarrow 0^{+}$;

(ii) or its projection on the $\lambda$ axis is $[0,+\infty)$.

Moreover, there exists $\bar{\lambda} \in(0,+\infty]$ such that, for every $\lambda \in(0, \bar{\lambda})$, the problem $\left(P_{\lambda}\right)$ has at least two $L^{p}$-viscosity solutions, $u_{\lambda, 1}$ and $u_{\lambda, 2}$, satisfying

$$
u_{\lambda, 1} \underset{\lambda \rightarrow 0^{+}}{\longrightarrow} u_{0} \text { in } C^{1}(\bar{\Omega}), \quad \max _{\bar{\Omega}} u_{\lambda, 2} \underset{\lambda \rightarrow 0^{+}}{\longrightarrow}+\infty
$$

and, if $\bar{\lambda}<+\infty$, the problem $\left(P_{\bar{\lambda}}\right)$ has a unique $L^{p}$-viscosity solution.

See section 5.1 for the main a priori and multiplicity theorems in the general form; section 5.2 and chapter 6 are devoted to their proofs. In the latter we construct and study an auxiliary fixed point problem in order to obtain the existence and nonexistence results via degree theory.

At the beginning of each chapter, a historical overview and references concerning the problem is provided, as well as the hypotheses and the precise statements. 


\section{2}

\section{Preliminaries}

In this chapter we recall some notation, definitions and known results (at least for specialists) that will be used throughout the thesis.

Let $\Omega$ be a bounded domain in $\mathbb{R}^{n}$ and $F(x, r, p, X): \Omega \times \mathbb{R} \times \mathbb{R}^{n} \times \mathbb{S}^{n} \rightarrow \mathbb{R}$ a measurable function. We consider the general structure condition

$$
\begin{array}{r}
\mathcal{M}_{\lambda, \Lambda}^{-}(X-Y)-b(x)|p-q|-\mu|p-q|(|p|+|q|)-d(x) \omega(|r-s|) \\
\leq F(x, r, p, X)-F(x, s, q, Y) \quad(S C)^{\mu} \\
\leq \mathcal{M}_{\lambda, \Lambda}^{+}(X-Y)+b(x)|p-q|+\mu|p-q|(|p|+|q|)+d(x) \omega(|r-s|)
\end{array}
$$

for a.e. $x \in \Omega$, where the operator $F$ satisfies $F(\cdot, 0,0,0) \equiv 0$ with positive ellipticity coefficients $\lambda \leq \Lambda, \mu$ is a nonnegative constant, $b \in L^{q}(\Omega), d \in L^{p}(\Omega)$, $b, d \geq 0$, for exponents $p$ and $q$ satisfying

$$
q \geq p \geq n, \quad q>n
$$

and $\omega$ is a modulus of continuity. As in [24], [25], hypothesis (2.1) is equivalent to the following two cases: (i) $p=q>n$ or (ii) $q>p=n$, that we are going to consider along the text. We use the same notation on exponents and on vectors in $(S C)^{\mu}$, as usual in the theory.

Notice that the assumption over the highest order term $X$, for $p=q$ and $r=s$, implies that $F$ is a uniformly elliptic operator; $F$ is uniformly continuous in $(r, p, X)$. In $(S C)^{\mu}$,

$$
\mathcal{M}_{\lambda, \Lambda}^{+}(X):=\sup _{\lambda I \leq A \leq \Lambda I} \operatorname{tr}(A X) \text { and } \mathcal{M}_{\lambda, \Lambda}^{-}(X):=\inf _{\lambda I \leq A \leq \Lambda I} \operatorname{tr}(A X)
$$

are the Pucci's extremal operators, which satisfy the following properties.

Lemma 2.1 (Lemma 2.1 in [12]) Let $X, Y \in \mathbb{S}^{n}$. Then,

(i) $\mathcal{M}_{\lambda, \Lambda}^{-}(X)=-\mathcal{M}_{\lambda, \Lambda}^{+}(-X)$;

(ii) $\mathcal{M}_{\lambda, \Lambda}^{-}(X)=\lambda \sum_{\left\{\nu_{i}>0\right\}} \nu_{i}+\Lambda \sum_{\left\{\nu_{i}<0\right\}} \nu_{i}, \mathcal{M}_{\lambda, \Lambda}^{+}(X)=\Lambda \sum_{\left\{\nu_{i}>0\right\}} \nu_{i}+$ $\lambda \sum_{\left\{\nu_{i}<0\right\}} \nu_{i}$, where $\left\{\nu_{i}\right\}_{1 \leq i \leq n}=\operatorname{spec}(X)$;

(iii) $\mathcal{M}_{\lambda, \Lambda}^{-}(X)+\mathcal{M}_{\lambda, \Lambda}^{-}(Y) \leq \mathcal{M}_{\lambda, \Lambda}^{-}(X+Y) \leq \mathcal{M}_{\lambda, \Lambda}^{-}(X)+\mathcal{M}_{\lambda, \Lambda}^{+}(X)$; 
(iv) $\mathcal{M}_{\lambda, \Lambda}^{-}(X)+\mathcal{M}_{\lambda, \Lambda}^{+}(Y) \leq \mathcal{M}_{\lambda, \Lambda}^{+}(X+Y) \leq \mathcal{M}_{\lambda, \Lambda}^{+}(X)+\mathcal{M}_{\lambda, \Lambda}^{+}(X)$;

(v) $\mathcal{M}_{\lambda a, \Lambda A}^{-}(X) \leq \mathcal{M}_{\lambda, \Lambda}^{-}(\phi X) \leq \mathcal{M}_{\lambda A, \Lambda a}^{-}(X)$, for $\phi(x) \in C(\bar{\Omega})$ such that $0<a \leq \phi(x) \leq A$.

For a proof see [26, p. 15], [27, p. 4] and lemma 4.2 in [28].

By modulus we mean a function $\omega:[0,+\infty] \rightarrow[0,+\infty]$ continuous at 0 with $\omega(0)=0$. We may consider $\omega$ increasing and continuous, up to replacing it by a larger function. We can also suppose $\omega$ subadditive, from where it holds the property $\omega(k) \leq(k+1) \omega(1)$ for all $k \geq 0$. More strongly, we will say that $\omega$ is a Lipschitz modulus if $\omega(k) \leq k \omega(1)$, for all $k \geq 0$.

In some cases, we are going to use the following linear structure condition

$$
\begin{array}{r}
\mathcal{M}_{\lambda, \Lambda}^{-}(X-Y)-b(x)|p-q|-d(x) \omega\left((r-s)^{+}\right) \\
\leq F(x, r, p, X)-F(x, s, q, Y) \\
\leq \mathcal{M}_{\lambda, \Lambda}^{+}(X-Y)+b(x)|p-q|+d(x) \omega\left((s-r)^{+}\right) \text {a.e. } x \in \Omega
\end{array}
$$

with $F(\cdot, 0,0,0) \equiv 0$, where $0<\lambda \leq \Lambda, b \in L^{p}(\Omega), d \in L^{p}(\Omega), b, d \geq 0$, for $p, q$ satisfying (2.1) and $\omega$ a modulus. Here, differently from $(S C)^{\mu}$, the condition over the zero order term in (SC) means that $F$ is proper (or coercive), i.e. decreasing in $r$.

Remark 2.2 Notice that equation (ME), with $L=F$ satisfying (SC), gives an example of an operator satisfying $(S C)^{\mu}$, for $\mu=\|M\|_{L^{\infty}(\Omega)}$. Indeed,

$$
\begin{aligned}
\langle M(x) p, p\rangle & -\langle M(x) q, q\rangle=\langle M(x) p, p\rangle+\langle M(x) p, q\rangle-\langle M(x) p, q\rangle-\langle M(x) q, q\rangle \\
& =\langle M(x) p, p-q\rangle+\langle M(x)(p-q), q\rangle \leq \mu|p-q|(|p|+|q|) .
\end{aligned}
$$

Here, $\|M(x)\|_{L^{\infty}(\Omega)}=\sup _{x \in \Omega}\|M(x)\|_{\mathbb{M}_{n \times n}(\mathbb{R})}$, for the matrix norm ${ }^{1}$

$$
\|M(x)\|_{\mathbb{M}_{n \times n}(\mathbb{R})}=\sup _{y \in \mathbb{R}^{n},|y|=1}|M(x) \cdot y|, \quad \text { for each } x \in \Omega .
$$

Next we recall the definition of $L^{p}$-viscosity solution. Here, solutions are real functions $u: \Omega \rightarrow \mathbb{R}$.

Definition 2.3 Let $f \in L_{l o c}^{p}(\Omega)$. We say that $u \in C(\Omega)$ is an $L^{p}$-viscosity subsolution (respectively, supersolution) of $F=f$ in $\Omega$ if for every $\phi \in W_{\mathrm{loc}}^{2, p}(\Omega)$

${ }^{1}$ Observe that both $\langle p, q\rangle$ and $p \cdot q$ denote the inner product of $p, q \in \mathbb{R}^{n}$. 
and $x_{0} \in \Omega$ such that $u-\phi$ has a local maximum (minimum) at $x_{0}$, one has

$$
\begin{gathered}
\text { ess } \varlimsup_{x \rightarrow x_{0}}\left\{F\left(x, u(x), D \phi(x), D^{2} \phi(x)\right)-f(x)\right\} \geq 0 \\
\left(\text { ess } \varliminf_{x \rightarrow x_{0}}\left\{F\left(x, u(x), D \phi(x), D^{2} \phi(x)\right)-f(x)\right\} \leq 0\right)
\end{gathered}
$$

in the sense that for every $\varepsilon, r>0$ there is a set $A \subset B_{r}\left(x_{0}\right)$ of positive measure such that $F\left(x, u(x), D \phi(x), D^{2} \phi(x)\right)-f(x) \geq-\varepsilon\left(F\left(x, u(x), D \phi(x), D^{2} \phi(x)\right)-\right.$ $f(x) \leq \varepsilon)$ for all $x \in A$.

In other words, whenever $\phi \in W_{\text {loc }}^{2, p}(\Omega), \varepsilon>0$ and $\mathcal{O} \subset \Omega$ open are such that $F\left(x, u(x), D \phi(x), D^{2} \phi(x)\right)-f(x) \leq-\varepsilon(\geq \varepsilon)$ for a.e. $x \in \mathcal{O}$, then $u-\phi$ cannot have a local maximum (minimum) in $\mathcal{O}$.

We can think about $L^{p}$-viscosity solutions for any $p>\frac{n}{2}$, since this restriction makes all test functions $\phi \in W_{\text {loc }}^{2, p}(\Omega)$ continuous [29, p. 284] and having a second order Taylor expansion [4]. We are going to deal mostly with the case $p>n$. In particular, for $\Omega$ bounded with $\partial \Omega \in C^{1,1}$, this implies that the continuous injection $W^{2, p}(\Omega) \subset C^{1}(\bar{\Omega})$ is compact, for all $n \geq 1$ [30, p. $213,285]$.

If $F$ and $f$ are continuous in $x$, we can use the more usual notion of $C$-viscosity sub and supersolutions. Precisely, we have the following definition.

Definition 2.4 Let $F(x, r, p, X): \Omega \times \mathbb{R} \times \mathbb{R}^{n} \times \mathbb{S}^{n} \rightarrow \mathbb{R}$ continuous and $f \in C(\Omega)$. We say that $u \in C(\Omega)$ is a $C$-viscosity subsolution (supersolution) of $F=f$ in $\Omega$ if for every $x_{0} \in \Omega$ and $\phi \in C^{2}\left(B_{r}\left(x_{0}\right)\right), r>0$, such that $u-\phi$ has a local maximum (minimum) at $x_{0}$, we have $F\left(x_{0}, u\left(x_{0}\right), D \phi\left(x_{0}\right), D^{2} \phi\left(x_{0}\right)\right) \geq$ $f\left(x_{0}\right)\left(F\left(x_{0}, u\left(x_{0}\right), D \phi\left(x_{0}\right), D^{2} \phi\left(x_{0}\right)\right) \leq f\left(x_{0}\right)\right)$.

Employing Taylor expansions of second order, we can replace the set of "test functions" in $C^{2}$ by polynomials of degree two [26, p. 13], or by an intrinsic approach on semi-jets [3, p. 9, 10]. We also refer to [31], [32], [33] for more properties and results concerning $C$-viscosity solutions.

Definitions 2.3 and 2.4 are equivalent when $F$ satisfies (SC) with $b \in$ $L^{\infty}(\Omega)$ and $p \geq n$, by theorem 2.1(ii) in [34] (which in turn goes back to proposition 2.9 in [4]) - we will be using them interchangeably in this case.

In both $L^{p}$ or $C$ cases, we only need to define a subsolution as an upper semicontinuous function, and a supersolution being lower semicontinuous, which is sufficient to ensure the attainment of a maximum or minimum, respectively, over compact sets. Moreover, if the function $u$ is not semicontinuous, we say that $u$ is a viscosity subsolution (supersolution) provided $u^{*}\left(u_{*}\right)$ is. Here, $u^{*}$ and $u_{*}$ are the upper and lower semicontinuous 
envelopes of $u$ respectively, namely $u^{*}(x):=\varlimsup_{y \rightarrow x} u(y), u_{*}(x):=\varliminf_{y \rightarrow x} u(y)$, see [3, p. 22], [33, p. 40]. Note that $u^{*}$ and $u_{*}$ are always semicontinuous functions with $u^{*} \in U S C(\Omega)$ and $u_{*} \in L S C(\Omega)$. Nevertheless, sub and supersolutions will be assumed continuous along the text, unless specifically noted otherwise.

A strong sub or subsolution belongs to $W_{\text {loc }}^{2, p}(\Omega)$ and satisfies the inequality at almost every point. These notions are related, up to quadratic growth, as shows the next proposition.

Proposition 2.5 Assume $F$ satisfies $(S C)^{\mu}, p, q$ as in $(2.1)$, and $f \in L^{p}(\Omega)$. Then, $u \in W_{\text {loc }}^{2, p}(\Omega)$ is a strong subsolution (supersolution) of $F=f$ in $\Omega$ if and only if it is an $L^{p}$-viscosity sub(super) solution of this equation.

See theorem 3.1 and proposition 9.1 in [25] for a proof, even for more general conditions on $\mu$ and the exponents $p, q$. Is is also well known that the pointwise maximum of subsolutions, or supremum over any set (for instance, if this supremum is locally bounded), is still a subsolution; see theorem 4.2 in [33] for $C$-viscosity solutions, and also proposition 2 in [35] for a version for $L^{p}$-viscosity solutions related to quadratic growth and bounded coefficients.

When we refer to solutions of the Dirichlet problem in $C^{1,1}$ domains, we will assume that strong solutions belong to $W^{2, p}(\Omega)$ - despite some cases in which we prefer to keep on the "local" sense in order to preserve the maximal generality. Remember that a solution is always both a sub and supersolution of the equation.

Denote $\mathcal{L}^{ \pm}[u]:=\mathcal{M}_{\lambda, \Lambda}^{ \pm}\left(D^{2} u\right) \pm b(x)|D u|$, where $b \in L_{+}^{q}(\Omega), q>n$. We make the convention that $b \in L_{+}^{p}(\Omega)$ when $p>n$ and no other information on $b$ or $q$ is provided.

Next we recall Alexandrov-Bakelman-Pucci type results with unbounded ingredients and quadratic growth, which will be referred simply by ABP.

Proposition 2.6 Let $\Omega$ bounded, $\mu \geq 0, b \in L_{+}^{q}(\Omega)$ and $f \in L^{p}(\Omega)$, for $p, q$ as in (2.1). Then there exist $\delta=\delta\left(n, p, \lambda, \Lambda\right.$, $\left.\operatorname{diam}(\Omega),\|b\|_{L^{q}(\Omega)}\right)>0$ such that

$$
\mu\left\|f^{-}\right\|_{L^{p}(\Omega)}(\operatorname{diam}(\Omega))^{\frac{n}{p}} \leq \delta \quad\left(\mu\left\|f^{+}\right\|_{L^{p}(\Omega)}(\operatorname{diam}(\Omega))^{\frac{n}{p}} \leq \delta\right)
$$

implies that every $u \in C(\bar{\Omega})$ which is an $L^{p}$-viscosity sub(super) solution of

$$
\mathcal{L}^{+}[u]+\mu|D u|^{2} \geq f(x) \text { in } \Omega^{+} \quad\left(\mathcal{L}^{-}[u]-\mu|D u|^{2} \leq f(x) \text { in } \Omega^{-}\right)
$$


where $\Omega^{ \pm}=\Omega \cap\{ \pm u>0\}$, satisfies the estimate

$$
\max _{\bar{\Omega}} u \leq \max _{\partial \Omega} u+C_{A}\left\|f^{-}\right\|_{L^{p}(\Omega)} \quad\left(\min _{\bar{\Omega}} u \geq \min _{\partial \Omega} u-C_{A}\left\|f^{+}\right\|_{L^{p}(\Omega)}\right),
$$

for a constant $C_{A}$ depending on $n, p, \lambda, \Lambda,\|b\|_{L^{q}(\Omega)}$, diam $(\Omega)$, which remains bounded if these quantities are bounded. Moreover, if $\mu=0$, the norm of $f$ on the right hand side can be taken in the $L^{n}$ space instead of $L^{p}$.

As a matter of fact, $\mathrm{ABP}$ is valid under more general conditions, even for unbounded $\mu$. We refer to proposition 2.8 in [36] for a complete proof in the case $\mu=0$. See theorem 2.6 and lemma 9.3 in [25], and theorem 3.4 in [37], for a precise dependence on constants (see also [24] and [36]). For a simplified proof for $\mu>0$ and $p>n$ (which is the only superlinear case that we need along the text), see appendix B.

A consequence of $\mathrm{ABP}$ in its quadratic form is the comparison principle for equations in the form (ME) (with $L=F$ there), concerning $L^{p}$-viscosity solutions and coercive operators. We make the convention, mainly when dealing with multiplicity results, that $\alpha$ and $\beta$ will always denote a pair of sub and supersolutions, in a sense to be specified.

Lemma 2.7 (Comparison Principle) Assume F satisfies (SC), $f \in L^{p}(\Omega)$ and $M \in L^{\infty}(\Omega)$ in the bounded domain $\Omega$. Suppose that $u$ is an $L^{p}$-viscosity supersolution of

$$
\left\{\begin{aligned}
-F[u] & =\langle M(x) D u, D u\rangle+f(x) & & \text { in } \Omega \\
u & =0 & & \text { on } \partial \Omega
\end{aligned}\right.
$$

Then, for any $\alpha \in C(\bar{\Omega}) \cap W_{\text {loc }}^{2, p}(\Omega)$ strong subsolution of (2.2), we have

$$
\alpha \leq u \text { in } \Omega
$$

Analogously, if $u$ is an $L^{p}$-viscosity subsolution of $(2.2)$, for any $\beta \in$ $C(\bar{\Omega}) \cap W_{\text {loc }}^{2, p}(\Omega)$ strong supersolution of $(2.2)$, we have $u \leq \beta$ in $\Omega$.

Proof. Set $v:=u-\alpha$ in $\Omega$. By contradiction, assume $\min _{\bar{\Omega}} v=v\left(x_{0}\right)<0$. As $v \geq 0$ on $\partial \Omega$, thus $x_{0} \in \Omega$. Set $\widetilde{\Omega}:=\{v<0$ in $\Omega\}$, which is an open nonempty set since $x_{0} \in \widetilde{\Omega}$. Let $\varphi \in W_{\text {loc }}^{2, p}(\widetilde{\Omega})$ and $\tilde{x} \in \widetilde{\Omega}$ be such that $v-\varphi$ has a minimum at $\tilde{x}$. But then $u-(\alpha+\varphi)$ has a minimum at $\tilde{x}$, and by $\alpha+\varphi \in W_{\text {loc }}^{2, p}(\widetilde{\Omega})$ together with the definition of $u$ being an $L^{p}$-viscosity supersolution, we know that for every $\varepsilon>0$, there exists $r>0$ such that, for a.e. $x \in B_{r}(\tilde{x}) \cap \widetilde{\Omega}$,

$$
-F\left(x, u, D \alpha+D \varphi, D^{2} \alpha+D^{2} \varphi\right)-\langle M(x) D(\alpha+\varphi), D(\alpha+\varphi)\rangle-f(x) \geq-\varepsilon
$$


and $-F\left(x, \alpha, D \alpha, D^{2} \alpha\right)-\langle M(x) D \alpha, D \alpha\rangle-f(x) \leq 0$ from the definition of $\alpha$ as strong subsolution. By (SC),

$$
\begin{aligned}
\varepsilon \geq & F\left(x, u, D \alpha+D \varphi, D^{2} \alpha+D^{2} \varphi\right)-F\left(x, \alpha, D \alpha, D^{2} \alpha\right) \\
& +\langle M(x) D(\alpha+\varphi), D(\alpha+\varphi)\rangle-\langle M(x) D \alpha, D \alpha\rangle \\
\geq & \mathcal{M}^{-}\left(D^{2} \varphi\right)-b(x)|D \varphi|-\mu(|D \alpha+D \varphi|+|D \alpha|)|D \varphi|-d(x) \omega\left((u-\alpha)^{+}\right) \\
\geq & \mathcal{M}^{-}\left(D^{2} \varphi\right)-b(x)|D \varphi|-\mu|D \varphi|^{2}-2 \mu|D \alpha||D \varphi| .
\end{aligned}
$$

Then, for $\widetilde{b}=b+2 \mu|D \alpha| \in L_{+}^{p}(\Omega), v$ is an $L^{p}$-viscosity supersolution of

$$
\left\{\begin{aligned}
\mathcal{M}^{-}\left(D^{2} v\right)-\widetilde{b}(x)|D v|-\mu|D v|^{2} & \leq 0 \text { in } \widetilde{\Omega} \\
v & \geq 0 \text { on } \partial \widetilde{\Omega} \subset \partial \Omega \cup\{v=0\} .
\end{aligned}\right.
$$

Thus, ABP with $f=0$ gives us $v \geq 0$ in $\widetilde{\Omega}$, contradicting the definition of $\widetilde{\Omega}$.

Remark 2.8 The same result holds if $\alpha=\max _{1 \leq i \leq m} \alpha_{i}$ and $\beta=\min _{1 \leq j \leq l} \beta_{j}$, where $\alpha_{i}$ and $\beta_{j}$ are continuous strong sub and supersolutions of (2.2) respectively. Indeed, in the proof above, we only need to note that $\min _{\bar{\Omega}}(u-\alpha)$ $=\left(u-\alpha_{i}\right)\left(x_{0}\right)$ for some $i \in\{1, \ldots, m\}$, and consider $v:=u-\alpha_{i}$.

For equations with linear growth in the gradient, it is also a consequence of $\mathrm{ABP}$ that the notions of $L^{p}$ and $L^{n}$ viscosity solutions coincide for $p \geq n$. This is the content of the next proposition, which is a version of theorem 2.1(iii) in [34] for unbounded coefficients.

Proposition 2.9 Suppose that $F$ satisfies (SC) for $b, d \in L^{q}(\Omega), q>n$, and let $f \in L_{\text {loc }}^{q}(\Omega)$. If $q \geq p \geq n$, then $u$ is an $L^{p}$-viscosity solution of $F \geq f$ in $\Omega$ if and only if $u$ is an $L^{q}$-viscosity solution of $F \geq f$ in $\Omega$.

For this, we need the following lemma, which is a version of proposition 1 in [35] for unbounded coefficients. The proof relies on an argument due to Prof. Boyan Sirakov.

Lemma 2.10 If F satisfies (SC) and (2.1), the maximum (minimum) in the definition of $L^{p}$-viscosity subsolution (supersolution) can be replaced by a strict maximum (minimum).

Proof. Suppose, in order to obtain a contradiction, that there exists $\varepsilon, r>0$ and $\phi \in W_{\text {loc }}^{2, p}(\Omega)$ such that $u-\phi$ attains a local maximum at $x_{0}$ but

$$
F\left(x, u(x), D \phi(x), D^{2} \phi(x)\right) \leq f(x)-\varepsilon \quad \text { a.e. in } B_{r}\left(x_{0}\right) .
$$


For simplicity of notation, suppose $x_{0}=0$ and $u-\phi \leq(u-\phi)(0)$ in $B_{2 r}(0)$.

Set $\phi_{\delta}:=\phi+\delta|x|^{4}+\psi_{\delta}$, where $\psi_{\delta}$ is the strong solution of

$$
\left\{\begin{aligned}
\mathcal{L}^{+}\left[\psi_{\delta}\right] & =-4 \delta b(x)|x|^{3}+\varepsilon / 8 & & \text { in } B_{r} \\
\psi_{\delta} & =0 & & \text { on } \partial B_{r}
\end{aligned}\right.
$$

given, for instance, by proposition 2.4 in [24]. Also, consider small values of $\delta \in(0,1)$ such that $4 \delta r^{2}(2+n \Lambda) \leq \varepsilon / 8$.

Claim 2.11 $u-\phi_{\delta}$ attains a strict local maximum in $B_{r}$ for small $\delta>0$.

Proof of Claim 2.11. Since $\mathcal{L}^{+}\left[\psi_{\delta} / \delta\right]=-4 b(x)|x|^{3}+\varepsilon /(4 \delta)=: g(x)$ a.e. in $B_{r}$, by ABP and Chebyshev's inequality $|\{|h|>t\}| \leq t^{-q}\|h\|_{L^{q}}^{q}$ for $h \in L^{q}(\Omega)$, it follows that

$$
\begin{aligned}
\frac{\psi_{\delta}}{\delta} & \leq C_{A}\left\|g^{-}\right\|_{L^{n}\left(B_{r}\right)} \leq C_{A}\left|B_{r} \cap\{g<0\}\right|^{\frac{1}{n}-\frac{1}{q}}\left\|g^{-}\right\|_{L^{q}\left(B_{r}\right)} \\
& \leq C_{A}\left|B_{r} \cap\left\{4 b(x)|x|^{3}>\varepsilon /(8 \delta)\right\}\right|^{\frac{1}{n}-\frac{1}{q}}\left\|g^{-}\right\|_{L^{q}\left(B_{r}\right)} \\
& \leq C(\delta / \varepsilon)^{\frac{q}{n}-1}\|b\|_{L^{q}(\Omega)}^{\frac{q}{n}-1}\left(\varepsilon+\|b\|_{L^{q}(\Omega)}\right) \underset{\delta \rightarrow 0^{+}}{\longrightarrow} 0
\end{aligned}
$$

for all $x \in B_{r}$, since $q>n$. In particular, $\psi_{\delta}(0)<\delta r^{4}$ for small $\delta>0$, so we have on $\partial B_{r}$ that $u-\phi_{\delta}=u-\phi-\delta r^{4}<(u-\phi)(0)-\psi_{\delta}(0)=\left(u-\phi_{\delta}\right)(0)$. Thus, $u-\phi_{\delta}$ must attain a strict local maximum at some point in $B_{r}$, say in the ball $B_{s}\left(x_{0}\right) \subset B_{r}(0)$, and the claim is proved.

Therefore, by applying the hypothesis over $\phi_{\delta}$,

$$
F\left(x, u(x), D \phi_{\delta}(x), D^{2} \phi_{\delta}(x)\right) \geq f(x)-\varepsilon / 2 \text { a.e. } x \in B_{s}\left(x_{0}\right) \text {. }
$$

So, using the latter and (2.3), we obtain

$$
\begin{aligned}
\varepsilon / 2 & \leq F\left(x, u(x), D \phi_{\delta}(x), D^{2} \phi_{\delta}(x)\right)-F\left(x, u(x), D \phi(x), D^{2} \phi(x)\right) \\
& \leq \mathcal{L}^{+}\left[\delta|x|^{4}+\psi_{\delta}\right] \leq 4 \delta|x|^{2}(2+n \Lambda)+4 \delta b(x)|x|^{3}+\mathcal{L}^{+}\left[\psi_{\delta}\right] \leq \varepsilon / 4
\end{aligned}
$$

a.e. $x \in B_{s}\left(x_{0}\right)$, by (2.4) and the choice of $\delta$, which yields a contradiction.

Proof of Proposition 2.9. It is obvious by definition 2.3 that $L^{p}$-viscosity solutions are $L^{q}$-viscosity, since $f \in L_{\text {loc }}^{q}(\Omega)$. So, in order to obtain a contradiction, suppose that $u$ is an $L^{q}$-viscosity but not $L^{p}$-viscosity subsolution of $F \geq f$ in $\Omega$. Then, there exists $\varphi \in W_{\text {loc }}^{2, p}(\Omega)$ and $B_{2 r}\left(x_{0}\right) \subset \Omega$ such that $u-\varphi$ has a local maximum at $x_{0}$ in $B_{r}\left(x_{0}\right)$ and

$$
F\left(x, u, D \varphi, D^{2} \varphi\right) \leq f(x)-\varepsilon \quad \text { a.e. in } B_{r}\left(x_{0}\right)
$$


We may assume that there exists $\delta>0$ such that $(u-\varphi)\left(x_{0}\right)=0$ and $u-\varphi \leq-\delta$ on $\partial B_{r}\left(x_{0}\right)$ by lemma 2.10 .

Let $\varphi_{k} \in C^{2}\left(B_{2 r}\left(x_{0}\right)\right)$ with $\varphi_{k} \rightarrow \varphi$ in $W_{\text {loc }}^{2, p} \subset W_{\text {loc }}^{2, n}$. Hence, $w_{k}:=u-\varphi_{k}$ is an $L^{q}$-viscosity subsolution of

$$
\mathcal{L}^{+}\left[w_{k}\right] \geq F\left(x, u, D u, D^{2} u\right)-F\left(x, u, D \varphi_{k}, D^{2} \varphi_{k}\right) \geq-g_{k}^{-}(x)
$$

in $B_{r}\left(x_{0}\right)$, for $g_{k}(x):=f(x)-F\left(x, u, D \varphi_{k}, D^{2} \varphi_{k}\right) \in L^{q}\left(B_{r}\right)$. Since by (2.5) we have $g_{k}^{-}(x)=-g_{k}(x) \leq F\left(x, u, D \varphi_{k}, D^{2} \varphi_{k}\right)-F\left(x, u, D \varphi, D^{2} \varphi\right) \leq \mathcal{L}^{+}\left[\varphi_{k}-\varphi\right]$ a.e. in $\left\{g_{k} \leq 0\right\} \cap B_{r}\left(x_{0}\right)$, then $\left\|g_{k}^{-}\right\|_{L^{n}\left(B_{r}\left(x_{0}\right)\right)}$ is bounded by

$$
(\lambda+\Lambda)\left\|D^{2}\left(\varphi_{k}-\varphi\right)\right\|_{L^{n}\left(B_{r}\left(x_{0}\right)\right)}+\|b\|_{L^{n}\left(B_{r}\left(x_{0}\right)\right)}\left\|D\left(\varphi_{k}-\varphi\right)\right\|_{L^{\infty}\left(B_{r}\left(x_{0}\right)\right)}
$$

which converges to zero when $k \rightarrow+\infty$, by the Sobolev continuous inclusion $W^{1, q} \subset L^{\infty}$ for $q>n$. Now it is just a question of applying ABP to obtain that $w_{k} \leq \sup _{\partial B_{r}\left(x_{0}\right)} w_{k}+\left\|g_{k}^{-}\right\|_{L^{n}\left(B_{r}\left(x_{0}\right)\right)}$ in $B_{r}\left(x_{0}\right)$ for all $k \in \mathbb{N}$. Thus, the uniform convergence of $\varphi_{k}$ to $\varphi$ in this last inequality is responsible for producing a contradiction with the definition of $\delta$.

The next proposition follows from theorem 4 in [12] in the case $p=n$. We refer to proposition 9.4 in [25] for a more general version.

Proposition 2.12 (Stability) Let $F, F_{k}$ operators satisfying $(S C)^{\mu}$ with $p, q$ as in (2.1), and $f, f_{k} \in L^{p}(\Omega)$. Let $u_{k} \in C(\Omega)$ be an $L^{p}$-viscosity subsolution (supersolution) of

$$
F_{k}\left(x, u_{k}, D u_{k}, D^{2} u_{k}\right) \geq f_{k}(x) \quad \text { in } \quad \Omega \quad(\leq) \quad \text { for all } k \in \mathbb{N} \text {. }
$$

Suppose $u_{k} \rightarrow u$ in $L_{\mathrm{loc}}^{\infty}(\Omega)$ as $k \rightarrow \infty$ and for each ball $B \subset \subset \Omega$ and $\varphi \in W^{2, p}(B)$, setting

$$
g_{k}(x):=F_{k}\left(x, u_{k}, D \varphi, D^{2} \varphi\right)-f_{k}(x) ; \quad g(x):=F\left(x, u, D \varphi, D^{2} \varphi\right)-f(x),
$$

we have $\left\|\left(g_{k}-g\right)^{+}\right\|_{L^{p}(B)}\left(\left\|\left(g_{k}-g\right)^{-}\right\|_{L^{p}(B)}\right) \rightarrow 0$ as $k \rightarrow \infty$. Then $u$ is an $L^{p}$-viscosity subsolution (supersolution) of $F\left(x, u, D u, D^{2} u\right) \geq f(x)(\leq)$ in $\Omega$.

If $F$ and $f$ are continuous in $x$, then it is enough that the above holds for every $\varphi \in C^{2}(B)$, in which case $u$ is a $C$-viscosity subsolution (supersolution) of $F=f$ in $\Omega$.

Remark 2.13 Proposition 2.12 is valid if we have $f_{k} \in L^{p}\left(\Omega_{k}\right), u_{k} \in C\left(\Omega_{k}\right)$, for an increasing sequence of domains $\Omega_{k} \subset \Omega_{k+1}$ such that $\Omega:=\bigcup_{k \in \mathbb{N}} \Omega_{k}$, see proposition 1.5 in [38]. 
The following proposition follows from the $C^{\beta}$ superlinear regularity established in [12]. We give the statement in terms of more general exponents $p, q$ as in (2.1), indicating the changes from the proof of theorem 2 there.

Proposition 2.14 ( $C^{\beta}$ Regularity) Let $\Omega \subset \mathbb{R}^{n}$ be a bounded domain. Assume $F$ satisfies $(S C)^{\mu}$ for $N=0, q=0, s=0$ and $b \in L_{+}^{q}(\Omega)$, for $p, q$ satisfying (2.1). Let $u \in C(\Omega)$ be an $L^{p}$-viscosity solution of (3.2) with $f \in L^{p}(\Omega)$. Then there exists $\beta \in(0,1)$ depending on $n, p, \lambda, \Lambda$ and $\|b\|_{L^{q}(\Omega)}$ such that $u \in C_{l o c}^{\beta}(\Omega)$ and for any subdomain $\Omega^{\prime} \subset \subset \Omega$ we have

$$
\|u\|_{C^{\beta}\left(\Omega^{\prime}\right)} \leq K_{1}\left\{\|u\|_{L^{\infty}(\Omega)}+\|f\|_{L^{p}(\Omega)}+\|d\|_{L^{p}(\Omega)} \omega\left(\|u\|_{L^{\infty}(\Omega)}\right)\right\}
$$

where $K_{1}$ depends only on $n, p, \lambda, \Lambda, \mu,\|b\|_{L^{q}(\Omega)},\|u\|_{L^{\infty}\left(\Omega^{\prime}\right)}$, $\operatorname{dist}\left(\Omega^{\prime}, \partial \Omega\right)$.

If, in addition, $u \in C(\bar{\Omega}) \cap C^{\tau}(\partial \Omega)$ and $\Omega$ satisfies a uniform exterior cone condition with size $L$, then there exists $\beta_{0}=\beta_{0}\left(n, p, \lambda, \Lambda, L,\|b\|_{L^{q}(\Omega)}\right) \in(0,1)$ and $\beta=\min \left(\beta_{0}, \frac{\tau}{2}\right)$ such that

$$
\|u\|_{C^{\beta}(\bar{\Omega})} \leq K_{1}\left\{\|u\|_{L^{\infty}(\Omega)}+\|f\|_{L^{p}(\Omega)}+\|u\|_{C^{\tau}(\partial \Omega)}+\|d\|_{L^{p}(\Omega)} \omega\left(\|u\|_{L^{\infty}(\Omega)}\right)\right\}
$$

where $K_{1}$ depends on $n, p, \lambda, \Lambda, \mu, L,\|b\|_{L^{q}(\Omega)}, \omega(1)\|d\|_{L^{p}(\Omega)}, \operatorname{diam}(\Omega),\|u\|_{L^{\infty}(\Omega)}$. In both cases, $K_{1}$ remains bounded if these quantities are bounded.

The same result holds if, instead of a solution of (3.2), $u$ is only an $L^{p}$-viscosity solution of the inequalities $\mathcal{L}^{-}[u]-\mu|D u|^{2} \leq g(x)$ and $\mathcal{L}^{+}[u]+$ $\mu|D u|^{2} \geq-g(x)$ in $\Omega$.

If $\mu=0$, then $K_{1}$ does not depend on a bound from above on $\|u\|_{L^{\infty}(\Omega)}$.

Proof. To obtain the statement in terms of $p$, it is only a question of reading $L^{n}$-viscosity sense in [12] as $L^{p}$-viscosity, changing $b \in L^{p}, d, f \in L^{n}$ there by $b \in L^{q}, d, f \in L^{p}$. The corresponding growth lemmas and exponents concerning $\rho$ must be replaced by $\rho^{1-\frac{n}{p}}$, which appear by using proposition 2.6 (for $\mu=0$ ) instead of theorem 3 there.

The zero order term is handled as part of the right hand side, since the whole proof is valid if we only have $u$ as an $L^{p}$-viscosity solution of inequalities $\mathcal{L}^{+}[u] \geq-g(x)$ and $\mathcal{L}^{-}[u] \leq g(x)$ in the case $\mu=0$ (see the final remark in the end of the proof of theorem 2 in [12]).

Next we recall two boundary versions of the quantitative strong maximum principle and the weak Harnack inequality, which follow by theorems 1.1 and 1.2 in [23] respectively.

We denote with $B_{r}^{+}=B_{r} \cap\left\{x_{n}>0\right\}$ a half ball with a flat portion of the boundary included in $\left\{x_{n}=0\right\}$, for $r>0$. Further, $\mathbb{T}_{r}:=B_{r} \cap\left\{x_{n}=0\right\}$. 
Theorem 2.15 (BQSMP) Let $d \in L^{p}\left(B_{2}^{+}\right), f \in L^{q}\left(B_{2}^{+}\right), p, q$ as in (2.1). Assume that $u$ is an $L^{p}$-viscosity supersolution of $\mathcal{L}^{-}[u]-d u \leq f, u \geq 0$ in $B_{2}^{+}$. Then there exist constants $\varepsilon, c, C>0$ depending on $n, \lambda, \Lambda, p, q,\|b\|_{L^{q}\left(B_{2}^{+}\right)}$ and $\|d\|_{L^{p}\left(B_{2}^{+}\right)}$such that

$$
\inf _{B_{1}^{+}} \frac{u}{x_{n}} \geq c\left(\int_{B_{3 / 2}^{+}}\left(f^{-}\right)^{\varepsilon}\right)^{1 / \varepsilon}-C\left\|f^{+}\right\|_{L^{q}\left(B_{2}^{+}\right)} .
$$

Theorem 2.16 (BWHI) Let $d \in L^{p}\left(B_{2}^{+}\right), f \in L^{q}\left(B_{2}^{+}\right), p, q$ as in (2.1). Assume that $u$ is an $L^{p}$-viscosity supersolution of $\mathcal{L}^{-}[u]-d u \leq f, u \geq 0$ in $B_{2}^{+}$. Then there exist constants $\varepsilon, c, C>0$ depending on $n, \lambda, \Lambda, p, q,\|b\|_{L^{q}\left(B_{2}^{+}\right)}$ and $\|d\|_{L^{p}\left(B_{2}^{+}\right)}$such that

$$
\inf _{B_{1}^{+}} \frac{u}{x_{n}} \geq c\left(\int_{B_{3 / 2}^{+}}\left(\frac{u}{x_{n}}\right)^{\varepsilon}\right)^{1 / \varepsilon}-C\left\|f^{+}\right\|_{L^{q}\left(B_{2}^{+}\right)} .
$$

In particular, theorem 2.16 implies the strong maximum principle when $f=0$, i.e. for an $L^{p}$-viscosity solution $u$ of $\mathcal{L}^{-}[u]-d u \leq 0, u \geq 0$ in $\Omega$, we have either $u \equiv 0$ in $\Omega$ or $u>0$ in $\Omega$ and if $u\left(x_{0}\right)=0$ at $x_{0} \in \partial \Omega$, then $\partial_{\nu} u\left(x_{0}\right)>0$. Here, $\partial_{\nu}$ is the derivative in the direction of the interior unit normal. We will refer to these consequences simply by SMP and Hopf, as below.

Proposition 2.17 (SMP) Let $\Omega$ be a $C^{1,1}$ domain and $u$ an $L^{p}$-viscosity solution of $\mathcal{L}^{-}[u]-d u \leq 0, u \geq 0$ in $\Omega$, where $d \in L^{p}(\Omega)$ and $p, q$ are as in (2.1). Then either $u>0$ in $\Omega$ or $u \equiv 0$ in $\Omega$.

Proposition 2.18 (Hopf) Let $\Omega$ be a $C^{1,1}$ domain and $u$ an $L^{p}$-viscosity solution of $\mathcal{L}^{-}[u]-d u \leq 0, u>0$ in $\Omega$, where $d \in L^{p}(\Omega)$ and $p, q$ are as in (2.1). If $u\left(x_{0}\right)=0$ for some $x_{0} \in \partial \Omega$, then $\partial_{\nu} u\left(x_{0}\right)>0$.

Notice that the results above generalize SMP and Hopf for $C$-viscosity solutions from [39]. In [23], theorems 2.15 and 2.16 (and consequently propositions 2.17 and 2.18) are proved for $d \equiv 0$, but exactly the same proofs there work for any $d \geq 0$. Moreover, since the function $u$ has a sign, they are also valid for nonproper operators, by splitting the positive and negative parts of $d$ and using $d^{-} u \geq 0$. We also refer to [40] for another proof of Hopf maximum principle.

The Local Maximum Principle (LMP) is well known in the literature, see for example [26, p. 36], [41, p. 244], [33, p. 85] and [42]. Its boundary version (BLMP) is given in theorem 1.3 in [23], without zero order term. 
Theorem 2.1 (BLMP) Assume that $u$ is a locally bounded $L^{p}$-viscosity subsolution of

$$
\left\{\begin{aligned}
\mathcal{L}^{+}[u]+\nu(x) u & \geq-f(x) & \text { in } B_{2}^{+} \\
u & \leq 0 & \text { on } \mathbb{T}_{2}
\end{aligned}\right.
$$

with $f \in L^{p}\left(B_{2}^{+}\right), b \in L_{+}^{q}\left(B_{2}^{+}\right), \nu \in L^{p_{1}}\left(B_{2}^{+}\right) \cap L^{p}\left(B_{2}^{+}\right)$, for some $p_{1}>n$ and $p, q$ as in (2.1). Then, for each $r>0$,

$$
\sup _{B_{1}^{+}} u^{+} \leq C\left(\left(\int_{B_{3 / 2}^{+}}\left(u^{+}\right)^{r}\right)^{1 / r}+\left\|f^{+}\right\|_{L^{p}\left(B_{2}^{+}\right)}\right)
$$

where $C$ depends only on $n, p, p_{1}, \lambda, \Lambda, r,\|b\|_{L^{q}\left(B_{2}^{+}\right)}$and $\|\nu\|_{L^{p_{1}\left(B_{2}^{+}\right)}}$.

Proof. As the proof of theorem 1.3 in [23], $v=u^{+}$can be extended as 0 in $B_{2} \backslash B_{2}^{+}$, since $v=0$ on $\mathbb{T}_{2}$ and satisfies (2.6) in $B_{2}$, with $f$ extended by 0 , which remains in $L^{p}\left(B_{2}\right)$. Then we apply theorem 2.2 below to obtain the boundary result.

Theorem 2.2 (LMP) Let $u$ be a locally bounded $L^{p}$-viscosity subsolution of $\mathcal{L}^{+}[u]+\nu(x) u \geq-f(x)$ in $B_{3}$, with $f \in L^{p}\left(B_{3}\right), \nu \in L^{p_{1}}\left(B_{3}\right) \cap L^{p}\left(B_{3}\right)$, for $p_{1}>n$ and $p, q$ as in (2.1). Then, for each $r>0$,

$$
\sup _{B_{1}} u^{+} \leq C\left(\left(\int_{B_{2}}\left(u^{+}\right)^{r}\right)^{1 / r}+\left\|f^{+}\right\|_{L^{p}\left(B_{3}\right)}\right)
$$

where $C$ depends only on $n, p, p_{1}, \lambda, \Lambda, r,\|b\|_{L^{q}\left(B_{3}\right)}$ and $\|\nu\|_{L^{p_{1}\left(B_{3}\right)}}$.

Remark 2.19 Of course the same conclusion holds if we have a domain $\Omega$ and $\Omega^{\prime} \subset \subset \Omega^{\prime \prime} \subset \subset \Omega$ by a local covering argument. The same for any radii $\rho_{1}<\rho_{2}<\rho_{3}$ by rescaling.

The only difference between theorem 2.2 and LMP given in theorem 3.1 (a) in [42] comes from the need to put the zero order term on the right hand side. For the sake of completeness, details are provided in the sequel.

Proof. Observe that $u$ is an $L^{n}$-viscosity subsolution of the initial equation by proposition 2.9, as well as $v:=u^{+}=\max (u, 0)$ of

$$
\mathcal{L}^{+}[v]+\nu(x) v \geq-f^{+}(x) \text { in } B_{3} .
$$


and so $\mathcal{L}^{+}[v] \geq-g(x)$, where $g:=f^{+}+\nu^{+} v \in L_{+}^{p}\left(B_{2}\right)$. Applying theorem 3.1(a) of [42] in the ball $B_{2}$, we obtain in particular that

$$
\sup _{B_{1}} v \leq C\left(\left(\int_{B_{3 / 2}} v^{s}\right)^{1 / s}+\|g\|_{L^{n}\left(B_{2}\right)}\right)
$$

for $s>n$ defined by the relation $\frac{1}{n}=\frac{1}{s}+\frac{1}{p_{1}}$. Then, Holder's inequality yields

$$
\left\|\nu^{+} v\right\|_{L^{n}\left(B_{2}\right)} \leq\left\|\nu^{+}\right\|_{L^{p_{1}\left(B_{2}\right)}}\|v\|_{L^{s}\left(B_{2}\right)}
$$

and for a constant depending also on $\left\|\nu^{+}\right\|_{L^{p_{1}\left(B_{2}\right)}}$, it follows that

$$
\sup _{B_{1}} v \leq C\left\{\|v\|_{L^{s}\left(B_{2}\right)}+\left\|f^{+}\right\|_{L^{n}\left(B_{2}\right)}\right\}
$$

Now we perform a Moser type argument, as in [32, p. 75], to extend the validity of (2.7) for all $s>0$. More precisely, we start rescaling (2.7). For $R \leq 2$, we define $w(x):=v(R x / 2)$ for $x \in B_{2}$ and apply the result just proved for $w$ to obtain, in terms of $v$,

$$
\sup _{B_{R / 2}} v \leq C\left\{R^{-\frac{n}{s}}\|v\|_{L^{s}\left(B_{R}\right)}+R\left\|f^{+}\right\|_{L^{n}\left(B_{R}\right)}\right\}
$$

Next, using (2.8) in $B=B_{(1-\theta) R}(y)$, for $R \in(0,2]$ and $\theta \in(0,1)$, we have

$$
\sup _{B_{(1-\theta) R / 2}(y)} v \leq C\left\{((1-\theta) R)^{-\frac{n}{s}}\|v\|_{L^{s}(B)}+(1-\theta) R\left\|f^{+}\right\|_{L^{n}(B)}\right\} .
$$

In particular, (2.9) for all $y \in B_{\theta R}$ yields, for $R \in(0,2]$ and $\theta \in(0,1)$,

$$
\|v\|_{L^{\infty}\left(B_{\theta R}\right)} \leq C\left\{((1-\theta) R)^{-\frac{n}{s}}\|v\|_{L^{s}\left(B_{R}\right)}+\left\|f^{+}\right\|_{L^{n}\left(B_{R}\right)}\right\}
$$

Let $r \in(0, s)$ (notice that the validity of BLMP to $r \geq s$ is ensured by (2.7) and Holder's inequality), then,

$$
\left(\int v^{s}\right)^{1 / s}=\left(\int v^{s-r} v^{r}\right)^{1 / s} \leq\|v\|_{\infty}^{1-\frac{r}{s}}\left(\int v^{r}\right)^{1 / s} .
$$

By Young's inequality with $\widetilde{p}=1 /(1-r / s)$ and $\widetilde{q}=s / r$,

$$
\begin{aligned}
\frac{C}{((1-\theta) R)^{n / s}}\|v\|_{L^{s}\left(B_{R}\right)} & \leq\|v\|_{L^{\infty}\left(B_{R}\right)}^{1-\frac{r}{s}}\left(\frac{C}{((1-\theta) R)^{n}} \int_{B_{R}} v^{r}\right)^{1 / s} \\
& \leq \frac{1}{2}\|v\|_{L^{\infty}\left(B_{R}\right)}+\left(\frac{C}{((1-\theta) R)^{n}} \int_{B_{R}} v^{r}\right)^{1 / r}
\end{aligned}
$$


Thus, applying this estimate in (2.10),

$$
\|v\|_{L^{\infty}\left(B_{\theta R}\right)} \leq \frac{1}{2}\|v\|_{L^{\infty}\left(B_{R}\right)}+\frac{C}{((1-\theta) R)^{n / r}}\left(\int_{B_{R}} v^{r}\right)^{1 / r}+C\left\|f^{+}\right\|_{L^{n}\left(B_{R}\right)} .
$$

Next, by defining $\psi(t):=\|v\|_{L^{\infty}\left(B_{t}\right)}, t \in(0,2]$, we have

$$
\psi(t) \leq \frac{1}{2} \psi(R)+\frac{C}{(R-t)^{n / r}}\left(\int_{B_{R}} v^{r}\right)^{1 / r}+C\left\|f^{+}\right\|_{L^{n}\left(B_{1}\right)} .
$$

Now, lemma 4.3 in [32] implies that $\psi(t) \leq \frac{C}{(R-t)^{n / r}}\left(\int_{B_{R}} v^{r}\right)^{1 / r}+C\left\|f^{+}\right\|_{L^{n}\left(B_{1}\right)}$. Taking $R \rightarrow 2^{-}$we obtain, for instance for $\theta=1 / 2$,

$$
\|v\|_{L^{\infty}\left(B_{1}\right)} \leq C\left(\left(\int_{B_{2}} v^{r}\right)^{1 / r}+\left\|f^{+}\right\|_{L^{n}\left(B_{2}\right)}\right) .
$$

We provide another proof of theorem 2.2 for $C$-viscosity solutions in appendix D, which is constructive and follows the classical proof in [41].

The following result, which follows from lemma 2.3 in [12], is a useful tool to deal with quadratic dependence in the gradient.

Lemma 2.20 (Exponential change) Let $p \geq n$ and $u \in W_{\mathrm{loc}}^{2, p}(\Omega)$. For $m>0$ set

$$
v=\frac{e^{m u}-1}{m}, \quad w=\frac{1-e^{-m u}}{m} .
$$

Then, a.e. in $\Omega$ we have $D v=(1+m v) D u, D w=(1-m w) D u$ and

$$
\begin{aligned}
& \mathcal{M}^{ \pm}\left(D^{2} u\right)+m \lambda|D u|^{2} \leq \frac{\mathcal{M}^{ \pm}\left(D^{2} v\right)}{1+m v} \leq \mathcal{M}^{ \pm}\left(D^{2} u\right)+m \Lambda|D u|^{2} \\
& \mathcal{M}^{ \pm}\left(D^{2} u\right)-m \Lambda|D u|^{2} \leq \frac{\mathcal{M}^{ \pm}\left(D^{2} w\right)}{1-m w} \leq \mathcal{M}^{ \pm}\left(D^{2} u\right)-m \lambda|D u|^{2}
\end{aligned}
$$

and clearly $\{u=0\}=\{v=0\}=\{w=0\}$ and $\{u>0\}=\{v>0\}=\{w>0\}$.

Moreover, the same inequalities hold in the $L^{p}$-viscosity sense if $u$ is merely continuous; for example, if $u \in C(\Omega)$ is an $L^{p}$-viscosity solution of

$$
\mathcal{M}^{+}\left(D^{2} u\right)+b(x)|D u|+\mu|D u|^{2}+c(x) u \geq f(x) \text { in } \Omega
$$

where $b \in L_{+}^{q}(\Omega), c, f \in L^{p}(\Omega)$, for $p, q$ as in $(2.1)$, then $v=\frac{1}{m}\left(e^{m u}-1\right)$, for $m=\frac{\mu}{\lambda}$, is an $L^{p}$-viscosity solution of

$$
\mathcal{M}^{+}\left(D^{2} v\right)+b(x)|D v|+\frac{c(x)}{m} \ln (1+m v)(1+m v)-m f(x) v \geq f(x) \text { in } \Omega
$$


and analogously for the other inequalities.

The proof follows the original idea of [12] for viscosity solutions, with the slight improvements that can be found in theorem 6.9 in [33]. See also the proof of lemma 1.6 in [43].

Proof. Inequalities (2.11) and (2.12) follow by a simple computation, by using $\operatorname{spec}(\xi \otimes \zeta)=\{0, \ldots, 0, \xi \cdot \zeta\}$, where $\xi \otimes \zeta \in \mathbb{M}_{n \times n}(\mathbb{R}),(\xi \otimes \zeta)_{i j}:=\xi_{i} \zeta_{j}$ for all ${ }^{2}$ $\xi, \zeta \in \mathbb{R}^{n}$. Suppose, then, that $u$ is an $L^{p}$-viscosity solution of (2.13).

Let $\psi \in W_{\text {loc }}^{2, p}(\Omega)$ such that $v-\psi$ attains a local maximum at $x_{0}$, namely $v \leq \psi$ and $v\left(x_{0}\right)=\psi\left(x_{0}\right)$. Let $\varepsilon>0$, take some $\Omega^{\prime}$ with $x_{0} \in \Omega^{\prime} \subset \subset \Omega$ and set $a=\|u\|_{L^{\infty}\left(\Omega^{\prime}\right)}$.

Define $\varphi=\frac{1}{m}\{\ln (1+m \psi)\} \in W_{\text {loc }}^{2, p}(\Omega)$, i.e. $\psi=\frac{1}{m}\left\{e^{m \varphi}-1\right\}$. Then, since $u-\varphi$ has a maximum at $x_{0}$, by using the definition of $u$ being an $L^{p}$-viscosity subsolution of (2.13) and also (2.11) for the pair $\varphi, \psi$, we get

$$
\frac{\mathcal{M}^{+}\left(D^{2} \psi\right)}{1+m \psi} \geq \mathcal{M}^{+}\left(D^{2} \varphi\right)+\mu|D u|^{2} \geq f(x)-b(x)|D \varphi|-c(x) u-\tilde{\varepsilon} \quad \text { a.e. in } \mathcal{O}
$$

where $\tilde{\varepsilon}=\frac{\varepsilon}{e^{a m}+1}$ and $\mathcal{O} \subset \Omega^{\prime}$ is some subset with positive measure.

Let $\delta \in(0,1)$. Notice that, since $(\psi-u)\left(x_{0}\right)=0$ and $\varphi \in W^{2, p}(\mathcal{O}) \subset$ $C(\overline{\mathcal{O}})$ for $p \geq n>n / 2$, there exists $\mathcal{O}_{\delta} \subset \mathcal{O}$ such that $v \leq \psi \leq v+\frac{\delta}{m}$ in $\mathcal{O}_{\delta}$. Thus $1+m \psi \leq 1+m v+\delta=e^{m u}+\delta$ and

$$
\begin{aligned}
& \mathcal{M}^{+}\left(D^{2} \psi\right)+b(x)|D \psi| \geq f^{+}(x) e^{m u}-f^{-}(x)\left(e^{m u}+\delta\right) \\
& \quad+\left(c^{+} u^{-}+c^{-} u^{+}\right) e^{m u}-\left(c^{+} u^{+}+c^{-} u^{-}\right)\left(e^{m u}+\delta\right)-\varepsilon \quad \text { a.e. in } \mathcal{O}_{\delta}
\end{aligned}
$$

i.e. we have shown that $v$ is an $L^{p}$-viscosity subsolution of

$$
\mathcal{M}^{+}\left(D^{2} v\right)+b(x)|D \psi| \geq f(x) e^{m u}-c(x) u e^{m u}-\left(f^{-}+c^{+} u^{+}+c^{-} u^{-}\right) \delta \quad \text { in } \Omega
$$

for any $\delta \in(0,1)$. The desired conclusion follows by letting $\delta \rightarrow 0$, since $\left\|f_{\delta}\right\|_{L^{p}(B)} \rightarrow 0$ for any $B \subset \subset \Omega$, where $f_{\delta}:=\left(f^{-}+c^{+} u^{+}+c^{-} u^{-}\right) \delta$, by proposition 2.12 . The proof of the remaining inequalities in the $L^{p}$-viscosity sense are similar.

A direct consequence of lemma 2.20 is the following extension of proposition 3.5 in [4]. Recall we say that $u$ is twice super(sub)differentiable

${ }^{2}$ Indeed, observe that if $A:=\xi \otimes \zeta=\xi \zeta^{T}$ and $\mathbb{R}^{n}$ is the direct sum of $V$ and $W$, for $V=\operatorname{span} \zeta, W=\operatorname{span}\left\{w_{i}\right\}_{i=1}^{n-1}$, then $A \xi=\xi\left(\zeta^{T} \xi\right)=(\xi \cdot \zeta) \xi$ and $A w_{i}=0$. Thus, $\xi \cdot \zeta$ is an eigenvalue with eigenvector $\xi$, while 0 is an eigenvalue with multiplicity $n-1$. 
at $x \in \Omega$ if there exists $(p, X) \in \mathbb{R}^{n} \times \mathbb{S}^{n}$ such that

$$
u(y) \leq(\geq) u(x)+p \cdot(y-x)+\frac{1}{2}\langle X(y-x), y-x\rangle+o\left(|y-x|^{2}\right) \text { as } y \rightarrow x .
$$

Corollary 2.21 Let $f \in L_{\text {loc }}^{p}(\Omega)$ for $p \geq n, \mu \geq 0, b \in L_{+}^{\infty}(\Omega)$ and $u$ a locally bounded $L^{p}$-viscosity solution of

$$
\mathcal{L}^{+}[u]+\mu|D u|^{2} \geq f(x) \quad \text { in } \Omega \quad\left(\mathcal{L}^{-}[u]-\mu|D u|^{2} \leq f(x) \quad \text { in } \Omega\right)
$$

Then $u$ is twice superdifferentiable (subdifferentiable) a.e. in $\Omega$.

Proof. Set $v=\frac{1}{m}\left(e^{m u}-1\right)$ for $m=\frac{\mu}{\lambda}$. Say $b(x) \leq \gamma$. Hence, by lemma 2.20 and $u \in L_{\mathrm{loc}}^{\infty}(\Omega), v$ is an $L^{p}$-viscosity solution of

$$
\mathcal{M}^{+}\left(D^{2} v\right)+\gamma|D v| \geq f(x)(1+m v) \in L_{\mathrm{loc}}^{p}(\Omega) .
$$

By proposition 3.5 in [4], $v$ is twice superdifferentiable a.e. in $\Omega$, and so is $u$.

It follows from the argument in [4], that locally bounded $L^{p}$-viscosity solutions of $F=f$, with $F$ satisfying the structure condition $(S C)^{\mu}$ for bounded coefficients, are twice differentiable a.e. and satisfy the equation at almost all points. Moreover, corollary 2.21 is true for $p>n-\varepsilon_{0}>n / 2$ as in [4], since lemma 2.20 also holds in this case - as well as most of the results stated here thanks to GMP; but not necessary for our purposes in this work.

We finish the chapter recalling some results about pure second order operators $F\left(D^{2} u\right)$, that is, uniformly elliptic operators $F$ depending only on $X$ (so Lipschitz continuous in $X$ ) and satisfying $F(0)=0$. These operators will play the role of $F(0,0,0, X)$ in the approximation lemmas. The next proposition is corollary 5.7 in [26], which deals with $C^{1, \bar{\alpha}}$ interior regularity.

Proposition 2.22 Let $u$ be a $C$-viscosity solution of $F\left(D^{2} u\right)=0$ in $B_{1}$. Then $u \in C^{1, \bar{\alpha}}\left(\bar{B}_{1 / 2}\right)$ for some universal $\bar{\alpha} \in(0,1)$ and there exists a constant $K_{2}$, depending on $n, \lambda$ and $\Lambda$, such that

$$
\|u\|_{C^{1, \bar{\alpha}}\left(\bar{B}_{1 / 2}\right)} \leq K_{2}\|u\|_{L^{\infty}\left(B_{1}\right)}
$$

We also need the following solvability result about the Dirichlet problem. 
Proposition 2.23 Let $\Omega$ bounded with a uniform exterior cone condition, $\psi \in C(\partial \Omega)$. Then there exists a unique $C$-viscosity solution $u \in C(\bar{\Omega})$ of

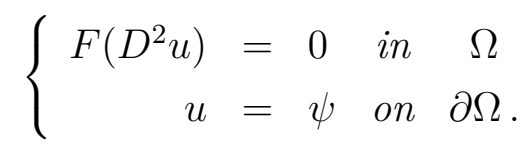

Proof. Uniqueness is corollary 5.4 in [26]. Let us recall how to obtain existence via Perron's Method, as in proposition II.1 in [44] (see also [45]). Indeed, comparison principle holds for $F\left(D^{2} u\right)$ by theorem 5.3 and corollary 3.7 in [26]. Further, we obtain a pair of strong sub and supersolutions $\underline{u}, \bar{u} \in$ $W_{\text {loc }}^{2, p}(\Omega) \cap C(\bar{\Omega})$ of Pucci's equations $\mathcal{M}^{+}\left(D^{2} \bar{u}\right) \leq 0 \leq \mathcal{M}^{-}\left(D^{2} \underline{u}\right)$ in $\Omega$ with $\underline{u}=\bar{u}=\psi$ on $\partial \Omega$ by lemma 3.1 of [4]. They are $L^{p}$-viscosity sub and supersolutions of $F\left(D^{2} u\right)=0$, from where we obtain the pair of $C$-viscosity sub and supersolutions desired.

We use the following notation from [38] and [46]. For $r, \nu>0$,

$$
B_{r}^{\nu}\left(x_{0}\right):=B_{r}\left(x_{0}\right) \cap\left\{x_{n}>-\nu\right\}, \quad \mathbb{T}_{r}^{\nu}\left(x_{0}\right):=B_{r}\left(x_{0}\right) \cap\left\{x_{n}=-\nu\right\} .
$$

Proposition 2.24 Let $u \in C\left(\overline{B_{1}^{\nu}}\right)$ be a C-viscosity solution of

$$
\left\{\begin{array}{rlll}
F\left(D^{2} u\right) & =0 & \text { in } & B_{1}^{\nu} \\
u & = & \text { on } & \mathbb{T}_{1}^{\nu}
\end{array}\right.
$$

such that $\psi \in C\left(\partial B_{1}^{\nu}\right) \cap C^{1, \tau}\left(\mathbb{T}_{1}^{\nu}\right)$ for some $\tau>0$. Then $u \in C^{1, \bar{\alpha}}\left(\overline{B_{1 / 2}^{\nu}}\right)$, where $\bar{\alpha}=\min \left(\tau, \alpha_{0}\right)$ for a universal $\alpha_{0}$. Moreover, for a constant $K_{3}$, depending only on $n, \lambda, \Lambda$ and $\tau$, we have

$$
\|u\|_{C^{1, \bar{\alpha}}\left(\overline{B_{1 / 2}^{\nu}}\right)} \leq K_{3}\left\{\|u\|_{L^{\infty}\left(B_{1}^{\nu}\right)}+\|\psi\|_{C^{1, \tau}\left(\mathbb{T}_{1}^{\nu}\right)}\right\}
$$

For a proof see proposition 2.2 in [46]; see also remark 3.3 in [38]. 


\section{3}

\section{Hölder Regularity}

The seminal work of Caffarelli [47] in 1989 brought an innovative approach of looking at Schauder type results via iterations from the differential quotients that are perturbations of solutions of the respective autonomous equations. The techniques in [47], which contains in particular $C^{1, \alpha}$ estimates for $L^{p}$-viscosity solutions of uniformly elliptic equations $F\left(x, D^{2} u\right)=f(x)$, allowed Święch [48] to extend them to more general operators $F\left(x, u, D u, D^{2} u\right)$ and later Winter [38] to boundary and global bounds. However, everything that is available in the literature, to our knowledge, for $L^{p}$-viscosity solutions in the fully nonlinear framework, concerns only structures with either linear gradient growth or bounded coefficients, except for some particular cases of extremal equations with small coefficients, see [24]. It is our goal here to obtain $C^{1, \alpha}$ regularity and estimates for general fully nonlinear uniformly elliptic equations, with at most quadratic growth in the gradient and unbounded coefficients.

We note that Trudinger, independently from [47], in [49] proved $C^{1, \alpha}$ regularity in a less general scenario than Święch and Winter, under a continuity hypothesis for $F$, dealing with $C$-viscosity solutions and approximations under supconvolutions. In that paper, it was stated that a priori estimates for solutions in $C^{1, \alpha}$ of superlinear equations could be derived from the arguments in [49] and [50]. However, the question of regularity is more complicated (for a discussion on differences between a priori bounds and regularity results we refer to [51]).

We also quote some other papers on $C^{1, \alpha}$ regularity, the classical works [52], [53], [54] for linear equations; [46] for Neumann boundary conditions; [55] for asymptotically convex operators; [56] (local) and [57] (global) for degenerate elliptic operators; [58] and [59] for parabolic equations possibly with VMO coefficients. Furthermore, Wang [43] has made an important contribution to $C^{1, \alpha}$ regularity for the parabolic equation $u_{t}+F\left(x, D^{2} u\right)=g(t, x, D u)$, where $|g(t, x, p)| \leq A|p|^{2}+g(t, x)$, for bounded coefficients, see lemma 1.6 in [43] (which uses theorem 4.19 in [60]). Sharp regularity results for general parabolic equations with linear gradient growth can be found in [61], and very complete $C^{1, \alpha}$ estimates on the boundary for solutions in the so called $S^{*}$-class for equations with linear gradient growth and unbounded coefficients in [62]. 
It is also essential to mention an important series of papers due to Koike and Święch [25], [36], [24], [63], in which they proved ABP and weak Harnack inequalities for $L^{p}$-viscosity solutions of equations with superlinear growth in the gradient, together with several theorems about existence, uniqueness and $W^{2, p}$ estimates for solutions of extremal equations involving Pucci's operators with unbounded coefficients, see in particular theorem 3.1 in [24]. Many of our arguments depend on the machinery in these works.

\section{1}

\section{Main Regularity Results}

In this section we present the hypotheses and statements of our results.

First, in order to measure the oscillation of $F$ in the $x$ entry, we define $\beta\left(x, x_{0}\right)=\beta_{F}\left(x, x_{0}\right)$, as in [47], [38],

$$
\beta\left(x, x_{0}\right):=\sup _{X \in \mathbb{S}^{n} \backslash\{0\}} \frac{\left|F(x, 0,0, X)-F\left(x_{0}, 0,0, X\right)\right|}{\|X\|} \text { a.e. } x, x_{0} .
$$

Notice that $\beta$ is a bounded function by $(S C)^{\mu}$ and lemma 2.1(ii). Next consider the usual hypothesis, as in [47], [38]: given $\theta>0$, there exists $r_{0}=r_{0}(\theta)>0$ such that

$$
\left(\frac{1}{r^{n}} \int_{B_{r}\left(x_{0}\right) \cap \Omega} \beta\left(x, x_{0}\right)^{p} \mathrm{~d} x\right)^{\frac{1}{p}} \leq \theta, \text { for all } r \leq r_{0} \text { a.e. } x_{0} .
$$

Notice that $\left(H_{\theta}\right)$ is satisfied for Pucci's extremal operators. Indeed, if for instance $F(x, r, p, X)=\mathcal{M}^{+}(X)+b(x)|p|+d(x) \omega\left(r^{-}\right)$, then $\beta_{F}\left(x, x_{0}\right) \equiv 0$. In particular this holds if $F$ is the linear operator $\operatorname{tr}(A(x) X)+b(x) \cdot p+d(x) r$, with $A$ a continuous matrix up to the boundary, as in chapter 1 .

The following is our main regularity result. To simplify its statement, here we assume that $\omega$ is a Lipschitz modulus, i.e. $\omega(r) \leq \omega(1) r$, for all $r \geq 0$.

Theorem 3.1 ( $C^{1, \alpha}$ Regularity Estimates) Let $\Omega \subset \mathbb{R}^{n}$ be a bounded domain. Assume $F$ satisfies $(S C)^{\mu}, f \in L^{p}(\Omega)$, where $p>n$. Let $u$ be an $L^{p}$-viscosity solution of

$$
F\left(x, u, D u, D^{2} u\right)=f(x) \quad \text { in } \Omega
$$

with $\|u\|_{L^{\infty}(\Omega)}+\|f\|_{L^{p}(\Omega)} \leq C_{0}$. Then, there exists $\alpha \in(0,1)$ and $\theta=\theta(\alpha)$, depending on $n, p, \lambda, \Lambda,\|b\|_{L^{p}(\Omega)}$, such that if $\left(H_{\theta}\right)$ holds for all $r \leq$ $\min \left\{r_{0}, \operatorname{dist}\left(x_{0}, \partial \Omega\right)\right\}$, for some $r_{0}>0$ and for all $x_{0} \in \Omega$, this implies that 
$u \in C_{\mathrm{loc}}^{1, \alpha}(\Omega)$ and for any subdomain $\Omega^{\prime} \subset \subset \Omega$,

$$
\|u\|_{C^{1, \alpha}\left(\overline{\Omega^{\prime}}\right)} \leq C\left\{\|u\|_{L^{\infty}(\Omega)}+\|f\|_{L^{p}(\Omega)}\right\}
$$

where $C$ depends only on $r_{0}, n, p, \lambda, \Lambda, \alpha, \mu,\|b\|_{L^{p}(\Omega)}, \omega(1)\|d\|_{L^{p}(\Omega)}, \operatorname{diam}(\Omega)$, $\operatorname{dist}\left(\Omega^{\prime}, \partial \Omega\right)$ and on the bound $C_{0}$.

If, in addition, $\partial \Omega \in C^{1,1}$ and $u \in C(\bar{\Omega}) \cap C^{1, \tau}(\partial \Omega)$ is such that and $\|u\|_{L^{\infty}(\Omega)}+\|f\|_{L^{p}(\Omega)}+\|u\|_{C^{1, \tau}(\partial \Omega)} \leq C_{1}$, then there exists $\alpha \in(0, \tau)$ and $\theta=\theta(\alpha)$, depending on $n, p, \lambda, \Lambda,\|b\|_{L^{p}(\Omega)}$, so that if $\left(H_{\theta}\right)$ holds for some $r_{0}>0$ and for all $x_{0} \in \bar{\Omega}$, this implies that $u \in C^{1, \alpha}(\bar{\Omega})$ and satisfies the estimate

$$
\|u\|_{C^{1, \alpha}(\bar{\Omega})} \leq C\left\{\|u\|_{L^{\infty}(\Omega)}+\|f\|_{L^{p}(\Omega)}+\|u\|_{C^{1, \tau}(\partial \Omega)}\right\}
$$

where $C$ depends on $r_{0}, n, p, \lambda, \Lambda, \alpha, \mu,\|b\|_{L^{p}(\Omega)}, \omega(1)\|d\|_{L^{p}(\Omega)}, \operatorname{diam}(\Omega), C_{1}$ and on the $C^{1,1}$ character of the boundary.

Moreover, if $\mu=0$, then the constant $C$ does not depend on $C_{0}, C_{1}$.

We also consider a slightly different (smaller) version of $\beta$, as in [48] and chapter 8 in $[26] ; \bar{\beta}\left(x, x_{0}\right)=\bar{\beta}_{F}\left(x, x_{0}\right)$ defined as

$$
\bar{\beta}\left(x, x_{0}\right):=\sup _{X \in \mathbb{S}^{n}} \frac{\left|F(x, 0,0, X)-F\left(x_{0}, 0,0, X\right)\right|}{\|X\|+1} \text { for all } x, x_{0} .
$$

Consider the hypothesis $(\bar{H})_{\theta}$, which is $\left(H_{\theta}\right)$ with $\beta$ replaced by $\bar{\beta}$. This hypothesis is trivially satisfied if $F(x, 0,0, X)$ is uniformly continuous in $x$, in the sense that there exists some modulus of continuity $\bar{\omega}$ such that

$$
\left|F(x, 0,0, X)-F\left(x_{0}, 0,0, X\right)\right| \leq \bar{\omega}\left(\left|x-x_{0}\right|\right)(\|X\|+1),
$$

for all $x, x_{0} \in \bar{\Omega}$ and $X \in \mathbb{S}^{n}$ (for instance, see [64]). See also remark 7.1 in [58] for a discussion over this hypothesis.

Remark 3.2 If $\mu=0$ we can replace the smallness condition $\left(H_{\theta}\right)$ by $(\bar{H})_{\theta}$ in the statement of Theorem 3.1, by adding 1 on the right hand side of (3.3) and (3.4), see remark 3.10 for details. For instance, in the global case,

$$
\|u\|_{C^{1, \alpha}(\bar{\Omega})} \leq C\left\{\|u\|_{L^{\infty}(\Omega)}+\|f\|_{L^{p}(\Omega)}+\|u\|_{C^{1, \tau}(\partial \Omega)}+1\right\}
$$

Remark 3.3 If $\omega$ is an arbitrary modulus, we still have regularity and estimates, with the same dependence on constants as before, by adding 1 on the right hand side of (3.3) and (3.4), as in (3.6). In this case, we can also obtain Theorem 3.1 in terms of $(\bar{H})_{\theta}$, see remark 3.11 . 
Of course, explicit zero order terms that only depend on $u$ and $x$, can always be handled as being part of the right hand side $f(x)$.

The proof of Theorem 3.1, given in section 3.2, is based on Caffarelli's iteration method. Compared to [48], [38], we use a simplified rescaling of variable which allows us to carry out the proof, without needing to use a twice differentiability property of viscosity solutions (whose validity is unknown for unbounded coefficients). We also use ideas of Wang to deal with superlinear terms.

The first application of the $C^{1, \alpha}$ theory is $W^{2, p}$ regularity for solutions of fully nonlinear equations with superlinear growth in the gradient, which are convex or concave in the variable $X$. This extends the results in [38] to superlinear growth in the gradient in the case $p>n$.

Theorem 3.4 ( $W^{2, p}$ Regularity) Let $\Omega \subset \mathbb{R}^{n}$ be a bounded domain and $u \in C(\Omega)$ an $L^{p}$-viscosity solution of

$$
F\left(x, u, D u, D^{2} u\right)+g(x, D u)=f(x) \text { in } \Omega
$$

where $f \in L^{p}(\Omega), p>n, g$ is a measurable function such that $g(x, 0)=0$ and $|g(x, p)-g(x, q)| \leq \gamma|p-q|+\mu|p-q|(|p|+|q|), F$ is convex or concave in $X$ satisfying $(S C)^{0}$, for $b, d \in L_{+}^{\infty}(\Omega)$ and $\omega$ a Lipschitz modulus. Also, suppose $\|u\|_{L^{\infty}(\Omega)}+\|f\|_{L^{p}(\Omega)} \leq C_{0}$. Then, there exists $\theta=\theta\left(n, p, \lambda, \Lambda,\|b\|_{L^{p}(\Omega)}\right)$ such that, if $\left(H_{\theta}\right)$ holds for all $r \leq \min \left\{r_{0}\right.$, $\left.\operatorname{dist}\left(x_{0}, \partial \Omega\right)\right\}$, for some $r_{0}>0$ and for all $x_{0} \in \Omega$, this implies that $u \in W_{\mathrm{loc}}^{2, p}(\Omega)$ and for every $\Omega^{\prime} \subset \subset \Omega$,

$$
\|u\|_{W^{2, p}\left(\Omega^{\prime}\right)} \leq C\left\{\|u\|_{L^{\infty}(\Omega)}+\|f\|_{L^{p}(\Omega)}\right\}
$$

where $C$ depends on $r_{0}, n, p, \lambda, \Lambda, \mu,\|b\|_{L^{p}(\Omega)}, \omega(1)\|d\|_{L^{p}(\Omega)}, \operatorname{dist}\left(\Omega^{\prime}, \partial \Omega\right), \operatorname{diam}(\Omega)$ and on the bound $C_{0}$.

If, moreover, $\partial \Omega \in C^{1,1}, u \in C(\bar{\Omega})$ and $u=\psi$ on $\partial \Omega$ for some $\psi \in W^{2, p}(\Omega)$ with $\|u\|_{L^{\infty}(\Omega)}+\|f\|_{L^{p}(\Omega)}+\|\psi\|_{W^{2, p}(\Omega)} \leq C_{1}$ then, there exists $\theta=\theta\left(n, p, \lambda, \Lambda,\|b\|_{L^{p}(\Omega)}\right)$ such that, if $\left(H_{\theta}\right)$ holds for some $r_{0}>0$ and for all $x_{0} \in \bar{\Omega}$, this implies that $u \in W^{2, p}(\Omega)$ and satisfies the estimate

$$
\|u\|_{W^{2, p}(\Omega)} \leq C\left\{\|u\|_{L^{\infty}(\Omega)}+\|f\|_{L^{p}(\Omega)}+\|\psi\|_{W^{2, p}(\Omega)}\right\}
$$

where $C$ depends on $r_{0}, n, p, \lambda, \Lambda, \mu,\|b\|_{L^{p}(\Omega)}, \omega(1)\|d\|_{L^{p}(\Omega)}, \operatorname{diam}(\Omega), C_{1}$ and on the $C^{1,1}$ character of the boundary.

Furthermore, if $\mu=0$, the constant $C$ does not depend on $C_{0}, C_{1}$. 
From the regularity and estimates related to $\mu=0$, we can give an alternative proof of proposition 2.4 in [24], about existence and uniqueness for the Pucci's extremal operators with unbounded coefficients in the case $p>n$.

Proposition 3.5 (Solvability of the Dirichlet problem) Let $\Omega \subset \mathbb{R}^{n}$ be a bounded $C^{1,1}$ domain. Let $b, d \in L_{+}^{p}(\Omega), p>n$ and $\omega$ a Lipschitz modulus. Let $f \in L^{p}(\Omega)$ and $\psi \in W^{2, p}(\Omega)$. Then, there exists $u_{ \pm} \in C(\bar{\Omega})$ which are the unique $L^{p}$-viscosity solutions of the problems

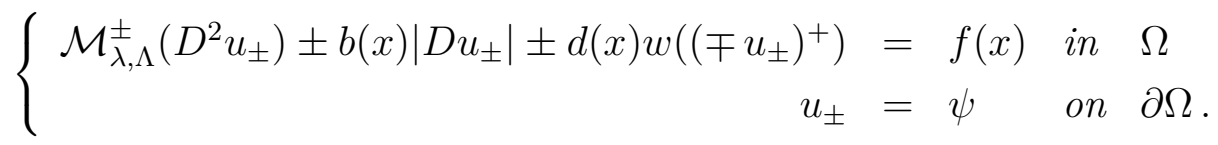

Moreover, $u_{ \pm} \in W^{2, p}(\Omega)$ and satisfies the estimate

$$
\left\|u_{ \pm}\right\|_{W^{2, p}(\Omega)} \leq C\left\{\left\|u_{ \pm}\right\|_{L^{\infty}(\Omega)}+\|f\|_{L^{p}(\Omega)}+\|\psi\|_{W^{2, p}(\Omega)}\right\}
$$

where $C$ depends only on $n, p, \lambda, \Lambda,\|b\|_{L^{p}(\Omega)}, \omega(1)\|d\|_{L^{p}(\Omega)}$, $\operatorname{diam}(\Omega)$ and on the $C^{1,1}$ character of the boundary.

On the other hand, as far as a priori bounds are concerned, we obtain the following version of Nagumo's lemma (for instance, a version of lemma 5.10 in $[65])$.

Lemma 3.6 (Generalized Nagumo's lemma) Let $\Omega \subset \mathbb{R}^{n}$ be a bounded $C^{1,1}$ domain. Let $F$ be a convex or concave operator in the $X$ entry, satisfying $(S C)^{\mu}$, with $b, d \in L_{+}^{p}(\Omega)$ for $p>n$ and $\omega$ an arbitrary modulus. Suppose that there exists $\theta>0$ such that $\left(H_{\theta}\right)$ holds for some $r_{0}>0$ and for all $x_{0} \in \bar{\Omega}$. Let $f \in L^{p}(\Omega), \psi \in W^{2, p}(\Omega)$ and let $u \in W^{2, p}(\Omega)$ be a strong solution of

$$
\left\{\begin{array}{rlrl}
F\left(x, u, D u, D^{2} u\right) & =f(x) \text { in } \Omega \\
u & =\psi & \text { on } & \partial \Omega
\end{array}\right.
$$

such that $\|u\|_{L^{\infty}(\Omega)}+\|f\|_{L^{p}(\Omega)}+\|\psi\|_{W^{2, p}(\Omega)} \leq C_{1}$. Then we have

$$
\|u\|_{W^{2, p}(\Omega)} \leq C\left\{\|u\|_{L^{\infty}(\Omega)}+\|f\|_{L^{p}(\Omega)}+\|\psi\|_{W^{2, p}(\Omega)}+\|d\|_{L^{p}(\Omega)} \omega\left(\|u\|_{L^{\infty}(\Omega)}\right)\right\}
$$

where $C$ depends on $r_{0}, n, p, \lambda, \Lambda, \mu,\|b\|_{L^{p}(\Omega)}$, $\operatorname{diam}(\Omega), C_{1}$ and on the $C^{1,1}$ character of the boundary. The local case is analogous.

Moreover, if $\mu=0$, the final constant $C$ does not depend on $C_{1}$.

Remark 3.7 Analogously to remark 3.2, we can replace $\left(H_{\theta}\right)$ by $(\bar{H})_{\theta}$ in Theorem 3.4 and Lemma 3.6 above, by adding 1 on the right hand side of the estimates, similar to (3.6). 


\section{2}

\section{Proof of theorem 3.1.}

In this section we give a detailed proof of theorem 3.1, splitting it into local, boundary and global parts, developed in subsections 3.2.1, 3.2.2 and 3.2 .3 , respectively.

\subsection{1}

\section{Local Regularity}

Fix a domain $\Omega^{\prime} \subset \subset \Omega$. Consider $K_{1}$ and $\beta$ the pair given by the $C^{\beta}$ local superlinear estimate (proposition 2.14) for $\Omega^{\prime}$, related to the initial $n, p, \lambda, \Lambda, \mu,\|b\|_{L^{p}(\Omega)}, \operatorname{dist}\left(\Omega^{\prime}, \partial \Omega\right)$ and $C_{0}$ such that

$$
\|u\|_{C^{\beta}\left(\Omega^{\prime}\right)} \leq K_{1}\left\{\|u\|_{L^{\infty}(\Omega)}+\|f\|_{L^{p}(\Omega)}+\|d\|_{L^{p}(\Omega)} \omega\left(\|u\|_{L^{\infty}(\Omega)}\right)\right\} .
$$

Also, let $K_{2}$ (which we can suppose greater than 1 ) and $\bar{\alpha}$ be the constants of $C^{1, \bar{\alpha}}$ local estimate (proposition 2.22) associated to $n, \lambda, \Lambda$ in the ball $B_{1}(0)$.

By taking $K_{1}$ larger and $\beta$ smaller, we can suppose $K_{1} \geq \widetilde{K}_{1}$ and $\beta \leq \widetilde{\beta}$, where $\widetilde{K}_{1}, \widetilde{\beta}$ is the pair of $C^{\beta}$ local estimate in the ball $B_{1}$ (or $B_{1 / 2}$ ), with respect to an equation with given constants $n, p, \lambda, \Lambda$ and bounds for the coefficients $\mu \leq 1,\|b\|_{L^{p}\left(B_{2}\right)} \leq 1+2 K_{2}\left|B_{1}\right|^{1 / p}$ and $\omega(1)\|d\|_{L^{p}\left(B_{2}\right)} \leq 1$, for all solutions in the ball $B_{2}$ with $\|u\|_{L^{\infty}\left(B_{2}\right)} \leq 1$ (or for all solutions in the ball $B_{1}$ with bounds on the coefficients in $B_{1}$ ).

The first step is to approximate our equation with one which already has the corresponding regularity and estimates that we are interested in.

Lemma 3.8 Assume $F$ satisfies $(S C)^{\mu}$ in $B_{1}, f \in L^{p}\left(B_{1}\right)$, where $p>n$. Let $\psi \in C^{\tau}\left(\partial B_{1}\right)$ with $\|\psi\|_{C^{\tau}\left(\partial B_{1}\right)} \leq K_{0}$. Then, for all $\varepsilon>0$, there exists $\delta \in(0,1)$, $\delta=\delta\left(\varepsilon, n, p, \lambda, \Lambda, \tau, K_{0}\right)$, such that

$$
\left\|\bar{\beta}_{F}(\cdot, 0)\right\|_{L^{p}\left(B_{1}\right)} \leq \delta,\|f\|_{L^{p}\left(B_{1}\right)} \leq \delta, \mu \leq \delta,\|b\|_{L^{p}\left(B_{1}\right)} \leq \delta, \omega(1)\|d\|_{L^{p}\left(B_{1}\right)} \leq \delta
$$

implies that any two $L^{p}$-viscosity solutions $v$ and $h$ of

$$
\left\{\begin{aligned}
F\left(x, v, D v, D^{2} v\right) & =f(x) & \text { in } & B_{1} \\
v & =\psi & \text { on } & \partial B_{1}
\end{aligned}\right.
$$

and

$$
\left\{\begin{aligned}
F\left(0,0,0, D^{2} h\right) & =0 \text { in } B_{1} \\
h & =\psi \text { on } \partial B_{1}
\end{aligned}\right.
$$

respectively, satisfy $\|v-h\|_{L^{\infty}\left(B_{1}\right)} \leq \varepsilon$. 
Proof. We are going to prove that for all $\varepsilon>0$, there exists a $\delta \in(0,1)$ satisfying the above, with $\delta \leq 2^{-\frac{n}{2 p}} \widetilde{\delta}^{1 / 2}$, where $\widetilde{\delta}$ is the constant from proposition 2.6. Assume the conclusion is not satisfied, then there exists some $\varepsilon_{0}>0$ and a sequence of operators $F_{k}$ satisfying $(S C)^{\mu_{k}}$ for $b_{k}, d_{k} \in L_{+}^{p}\left(B_{1}\right)$, $\mu_{k} \geq 0, \omega_{k}$ modulus, $f_{k} \in L^{p}\left(B_{1}\right)$ and $\delta_{k} \in(0,1)$ such that $\delta_{k} \leq 2^{-\frac{n}{2 p}} \widetilde{\delta}_{k}^{1 / 2}$ for all $k \in \mathbb{N}$, where $\widetilde{\delta}_{k}$ is the number from ABP related to $b_{k}$, in addition to

$$
\left\|\bar{\beta}_{F_{k}}(\cdot, 0)\right\|_{L^{p}\left(B_{1}\right)},\left\|f_{k}\right\|_{L^{p}\left(B_{1}\right)}, \mu_{k},\left\|b_{k}\right\|_{L^{p}\left(B_{1}\right)}, \omega_{k}(1)\left\|d_{k}\right\|_{L^{p}\left(B_{1}\right)} \leq \delta_{k} \underset{k \rightarrow \infty}{\longrightarrow} 0
$$

with $v_{k}, h_{k} \in C\left(\bar{B}_{1}\right) L^{p}$-viscosity solutions of

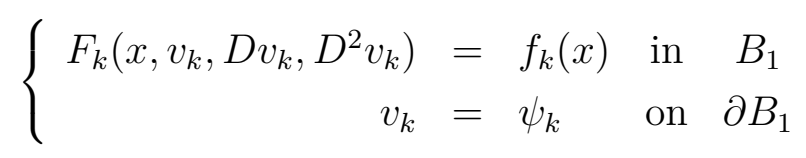

and

$$
\left\{\begin{aligned}
F_{k}\left(0,0,0, D^{2} h_{k}\right) & =0 \quad \text { in } \quad B_{1} \\
h_{k} & =\psi_{k} \text { on } \partial B_{1}
\end{aligned}\right.
$$

where $\left\|\psi_{k}\right\|_{C^{\tau}\left(\partial B_{1}\right)} \leq K_{0}$, but $\left\|v_{k}-h_{k}\right\|_{L^{\infty}\left(B_{1}\right)}>\varepsilon_{0}$. We first claim that

$$
\left\|v_{k}\right\|_{L^{\infty}\left(B_{1}\right)},\left\|h_{k}\right\|_{L^{\infty}\left(B_{1}\right)} \leq C_{0}
$$

for large $k$, where $C_{0}=C_{0}\left(n, p, \lambda, \Lambda, K_{0}\right)$. Indeed, in the first place, since we have $\mathcal{M}^{-}\left(D^{2} h_{k}\right) \leq 0 \leq \mathcal{M}^{+}\left(D^{2} h_{k}\right)$ in the viscosity sense, we obtain directly that $\left\|h_{k}\right\|_{L^{\infty}\left(B_{1}\right)} \leq\left\|\psi_{k}\right\|_{L^{\infty}\left(\partial B_{1}\right)} \leq K_{0}$. For $v_{k}$, we initially observe that

$$
2^{\frac{n}{p}} \mu_{k} \delta_{k} \leq 2^{\frac{n}{p}} \delta_{k}^{2} \leq \widetilde{\delta}_{k}, \quad \text { for all } k \in \mathbb{N}
$$

Further, $v_{k}$ is an $L^{p}$-viscosity solution of

$$
\mathcal{L}_{k}^{+}\left[v_{k}\right]+\mu_{k}\left|D v_{k}\right|^{2}+d_{k}(x) \omega_{k}\left(\left|v_{k}\right|\right) \geq f_{k}(x) \geq \mathcal{L}_{k}^{-}\left[v_{k}\right]-\mu_{k}\left|D v_{k}\right|^{2}-d_{k}(x) \omega_{k}\left(\left|v_{k}\right|\right)
$$

Then, applying ABP in its quadratic form in $B_{1}$, we obtain, as in [38], that

$$
\left\|v_{k}\right\|_{L^{\infty}\left(B_{1}\right)} \leq\left\|v_{k}\right\|_{L^{\infty}\left(\partial B_{1}\right)}+C_{A}^{k}\left\{\left\|f_{k}\right\|_{L^{p}\left(B_{1}\right)}+\left\|d_{k}\right\|_{L^{p}} \omega_{k}(1)\left(\left\|v_{k}\right\|_{L^{\infty}\left(B_{1}\right)}+1\right)\right\}
$$

Since $\left\|b_{k}\right\|_{L^{n}\left(B_{1}\right)} \leq\left|B_{1}\right|^{\frac{p-n}{n p}}$ for large $k$, then the constant in $\mathrm{ABP}$ is uniformly bounded, say $C_{A}^{k} \leq C_{A}$. Using also that $\left\|f_{k}\right\|_{L^{p}\left(B_{1}\right)} \leq 1$ and $C_{A} \omega_{k}(1)\left\|d_{k}\right\|_{L^{p}(\Omega)} \leq 1 / 2$ for large $k$, we obtain that $\left\|v_{k}\right\|_{L^{\infty}\left(B_{1}\right)} \leq C_{0}$, with $C_{0}=C_{0}\left(n, p, \lambda, \Lambda, K_{0}\right)$, proving the claim (3.8).

Then, by the $C^{\beta}$ global estimate (proposition 2.14), there exists some 
$\beta \in(0,1)$ such that

$$
\left\|v_{k}\right\|_{C^{\beta}\left(\bar{B}_{1}\right)},\left\|h_{k}\right\|_{C^{\beta}\left(\bar{B}_{1}\right)} \leq C
$$

where $\beta=\min \left(\beta_{0}, \frac{\tau}{2}\right)$ for some $\beta_{0}=\beta_{0}(n, p, \lambda, \Lambda), C=C\left(n, p, \lambda, \Lambda, C_{0}\right)$. Here, $\beta$ and $C$ do not depend on $k$, since $\mu_{k},\left\|b_{k}\right\|_{L^{p}\left(B_{1}\right)}, \omega_{k}(1)\left\|d_{k}\right\|_{L^{p}\left(B_{1}\right)},\left\|f_{k}\right\|_{L^{p}\left(B_{1}\right)} \leq$ 1 for all $k \in \mathbb{N}$. Then, by the compact inclusion $C^{\beta}\left(\bar{B}_{1}\right) \subset C\left(\bar{B}_{1}\right)$ we have, up to subsequences, that

$$
v_{k} \longrightarrow v_{\infty}, \quad h_{k} \longrightarrow h_{\infty} \quad \text { in } C\left(\bar{B}_{1}\right) \quad \text { as } k \rightarrow \infty
$$

for some $v_{\infty}, h_{\infty} \in C\left(\bar{B}_{1}\right)$ with $v_{\infty}=h_{\infty}=\psi_{\infty}$ on $\partial B_{1}$. Moreover, by Arzelà-Ascoli theorem, a subsequence of $F_{k}(0,0,0, X)$ converges uniformly on compact sets of $\mathbb{S}^{n}$ to some uniformly elliptic operator $F_{\infty}(X)$, since $\mathcal{M}_{\lambda, \Lambda}^{-}(X-Y) \leq 0=F_{k}(0,0,0, X)-F_{k}(0,0,0, Y) \leq \mathcal{M}_{\lambda, \Lambda}^{+}(X-Y)$.

We claim that both $v_{\infty}$ and $h_{\infty}$ are viscosity solutions of

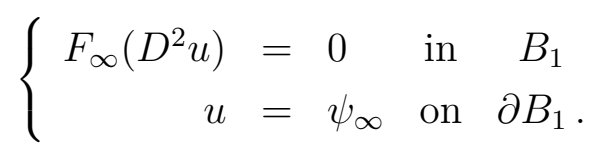

This implies that they are equal, by proposition 2.23 , which contradicts $\left\|v_{\infty}-h_{\infty}\right\|_{L^{\infty}\left(B_{1}\right)} \geq \varepsilon_{0}$.

The claim for $h_{\infty}$ follows by passing to uniform limits in the equation satisfied by $h_{k}$. On the other hand, for $v_{\infty}$ we apply stability (proposition 2.12) by noticing that, for $\varphi \in C^{2}\left(B_{1}\right)$,

$$
\begin{aligned}
F_{k}\left(x, v_{k}, D \varphi, D^{2} \varphi\right)- & f_{k}(x)-F_{\infty}\left(D^{2} \varphi\right) \\
=\{ & \left.F_{k}\left(x, v_{k}, D \varphi, D^{2} \varphi\right)-F_{k}\left(x, 0,0, D^{2} \varphi\right)\right\} \\
& \quad+\left\{F_{k}\left(x, 0,0, D^{2} \varphi\right)-F_{k}\left(0,0,0, D^{2} \varphi\right)\right\} \\
& +\left\{F_{k}\left(0,0,0, D^{2} \varphi\right)-F_{\infty}\left(D^{2} \varphi\right)\right\}-f_{k}(x)
\end{aligned}
$$

and that each one of the addends in braces tends to zero in $L^{p}\left(B_{1}\right)$ as $k \rightarrow \infty$. Indeed, the first one in modulus is less or equal than $\mu_{k}|D \varphi(x)|^{2}+$ $b_{k}(x)|D \varphi(x)|+\omega_{k}\left(\left\|v_{k}\right\|_{L^{\infty}\left(B_{1}\right)}\right) d_{k}(x)$, so its $L^{p}$-norm is bounded by

$$
\mu_{k}\|D \varphi\|_{L^{\infty}\left(B_{1}\right)}^{2}+\left\|b_{k}\right\|_{L^{p}\left(B_{1}\right)}\|D \varphi\|_{L^{\infty}\left(B_{1}\right)}+\left(C_{0}+1\right) \omega_{k}(1)\left\|d_{k}\right\|_{L^{p}\left(B_{1}\right)} ;
$$

while the $L^{p}$-norm of the second and third are bounded by

$$
\left\|\bar{\beta}_{F_{k}}(\cdot, 0)\right\|_{L^{p}\left(B_{1}\right)}\left(\left\|D^{2} \varphi\right\|_{L^{\infty}\left(B_{1}\right)}+1\right)
$$


and

$$
\left|B_{1}\right|^{1 / p}\left\|F_{k}\left(0,0,0, D^{2} \varphi\right)-F_{\infty}\left(D^{2} \varphi\right)\right\|_{L^{\infty}\left(B_{1}\right)}
$$

respectively, what concludes the proof.

Proof of Local Regularity Estimates in the set $\Omega^{\prime}$. The main difference from the case $\mu=0$, in the present proof, consists of defining a slightly different scaling on the function, which allows us to have $\mu$ small in order to obtain the conditions of the approximation lemma 3.8. For this, we will bring forward an argument due to Wang [43], that uses the $C^{\beta}$ regularity of $u$.

Set $W:=\|u\|_{L^{\infty}(\Omega)}+\|f\|_{L^{p}(\Omega)}+\|d\|_{L^{p}(\Omega)} \omega\left(\|u\|_{L^{\infty}(\Omega)}\right)$, which is less or equal than $W_{0}$, a constant that depends on $C_{0}$ and $\omega(1)\|d\|_{L^{p}(\Omega)}$.

For ease of notation, assume $0 \in \Omega^{\prime}$ and set $s_{0}:=\min \left(r_{0}, \operatorname{dist}\left(0, \partial \Omega^{\prime}\right)\right)$. Recall that this $r_{0}=r_{0}(\theta)$ is such $\left(H_{\theta}\right)$ holds for all $r \leq \min \left\{r_{0}, \operatorname{dist}\left(x_{0}, \partial \Omega\right)\right\}$, for all $x_{0} \in \Omega$. We will see, in the sequel, how the choice of $\theta$ is done.

We start assigning some constants. Fix an $\alpha \in(0, \bar{\alpha})$ with $\alpha \leq \min (\beta, 1-$ $n / p)$. Then, choose $\gamma=\gamma\left(\alpha, \bar{\alpha}, K_{2}\right) \in\left(0, \frac{1}{4}\right]$ such that

$$
2^{2+\bar{\alpha}} K_{2} \gamma^{\bar{\alpha}} \leq \gamma^{\alpha}
$$

and define

$$
\varepsilon=\varepsilon(\gamma):=K_{2}(2 \gamma)^{1+\bar{\alpha}}
$$

This $\varepsilon$ provides a $\delta=\delta(\varepsilon) \in(0,1)$, the constant of the approximation lemma 3.8 that, up to diminishing, can be supposed to satisfy

$$
\left(5+2 K_{2}\right) \delta \leq \gamma^{\alpha}
$$

Now let $\sigma=\sigma\left(s_{0}, n, p, \alpha, \bar{\alpha}, \beta, \delta, \mu,\|b\|_{L^{p}(\Omega)}, \omega(1)\|d\|_{L^{p}(\Omega)}, K_{1}, K_{2}, C_{0}\right) \leq \frac{s_{0}}{2}$ such that

$$
\sigma^{\min \left(1-\frac{n}{p}, \beta\right)} m \leq \delta\left\{32 K^{2}\left(K_{2}+K+1\right)\left|B_{1}\right|^{1 / p}\right\}^{-1}
$$

where $m:=\max \left\{1,\|b\|_{L^{p}(\Omega)}, \omega(1)\|d\|_{L^{p}(\Omega)}, \mu\left(1+2^{\beta} K_{1}\right) W_{0}\right\}$. Consider the constant $K\left(\gamma, \alpha, K_{2}\right)$ defined as $K=K_{2} \gamma^{-\alpha}\left(1-\gamma^{\alpha}\right)^{-1}+K_{2} \gamma^{-1-\alpha}\left(1-\gamma^{1+\alpha}\right)^{-1}$ which is greater than $K_{2} \geq 1$. In particular, $\bar{B}_{2 \sigma}(0) \subset \Omega^{\prime}$ and we can define

$$
N=N_{\sigma}(0):=\sigma W+\sup _{x \in B_{2}}|u(\sigma x)-u(0)| .
$$


By construction and $C^{\beta}$ local quadratic estimate, $N$ is uniformly bounded by

$$
\sigma W \leq N \leq\left(\sigma+2^{\beta} K_{1} \sigma^{\beta}\right) W \leq\left(1+2^{\beta} K_{1}\right) W_{0} \sigma^{\beta}
$$

Claim $3.9 \widetilde{u}(x):=\frac{1}{N}\{u(\sigma x)-u(0)\}$ is an $L^{p}$-viscosity solution of $\widetilde{F}[\widetilde{u}]=$ $\tilde{f}(x)$ in $B_{2}$, where

$$
\widetilde{F}(x, r, p, X):=\frac{\sigma^{2}}{N} F\left(\sigma x, N r+u(0), \frac{N}{\sigma} p, \frac{N}{\sigma^{2}} X\right)-\frac{\sigma^{2}}{N} F(\sigma x, u(0), 0,0)
$$

and $\tilde{f}:=\widetilde{f}_{1}+\widetilde{f}_{2}$ for

$$
\tilde{f}_{1}(x):=\sigma^{2} f(\sigma x) / N, \quad \widetilde{f}_{2}(x):=-\sigma^{2} F(\sigma x, u(0), 0,0) / N,
$$

with $\widetilde{F}$ satisfying $(\widetilde{S C})^{\tilde{\mu}}$ for $\widetilde{b}(x):=\sigma b(\sigma x), \widetilde{\mu}:=N \mu, \widetilde{d}(x):=\sigma^{2} d(\sigma x)$ and $\widetilde{\omega}(r):=\omega(N r) / N$.

Proof of Claim 3.9. Let $\varepsilon>0$ and $\widetilde{\varphi} \in W_{\text {loc }}^{2, p}\left(B_{2}\right)$ such that $\widetilde{u}-\widetilde{\varphi}$ has a minimum (maximum) at $x_{0} \in B_{2}$. Define $\varphi(x):=N \widetilde{\varphi}(x / \sigma)+u(0)$ in $B_{2 \sigma}(0)$ and notice that $u-\varphi$ has a minimum (maximum) at $\sigma x_{0} \in B_{2 \sigma}$. Since $u$ is an $L^{p}$-viscosity solution on $B_{2 \sigma}$, for this $\varepsilon>0$ there exists $r>0$ such that

$$
F\left(\sigma x, u(\sigma x), D \varphi(\sigma x), D^{2} \varphi(\sigma x)\right) \leq(\geq) f(\sigma x)+(-) N \varepsilon / \sigma^{2} \quad \text { a.e. in } B_{r}\left(x_{0}\right),
$$

which is equivalent to

$$
\frac{\sigma^{2}}{N} F\left(\sigma x, N \widetilde{u}(x)+u(0), \frac{N}{\sigma} D \widetilde{\varphi}(x), \frac{N}{\sigma^{2}} D^{2} \widetilde{\varphi}(x)\right) \leq(\geq) \frac{\sigma^{2}}{N} f(\sigma x)+(-) \varepsilon
$$

a.e. in $B_{r}\left(x_{0}\right)$. Adding $-\sigma^{2} F(\sigma x, u(0), 0,0) / N$ in both sides, we have

$$
\widetilde{F}\left(x, \widetilde{u}(x), D \widetilde{\varphi}(x), D^{2} \widetilde{\varphi}(x)\right) \leq(\geq) \widetilde{f}(x)+(-) \varepsilon \quad \text { a.e. in } B_{r}\left(x_{0}\right)
$$

Further, $\widetilde{F}(x, 0,0,0)=0$ a.e. $x \in B_{2}$ and for all $r \in \mathbb{R}, p \in \mathbb{R}^{n}, X \in \mathbb{S}^{n}$,

$$
\begin{aligned}
\widetilde{F} & (x, r, p, X)-\widetilde{F}(x, s, q, Y) \\
= & \frac{\sigma^{2}}{N}\left\{F\left(\sigma x, N r+u(0), \frac{N}{\sigma} p, \frac{N}{\sigma^{2}} X\right)-F\left(\sigma x, N s+u(0), \frac{N}{\sigma} q, \frac{N}{\sigma^{2}} Y\right)\right\} \\
\leq & \mathcal{M}_{\lambda, \Lambda}^{+}(X-Y)+\sigma b(\sigma x)|p-q|+N \mu|p-q|(|p|+|q|) \\
\quad & \quad+\sigma^{2} d(\sigma x) \omega(N|r-s|) / N \\
= & \mathcal{M}_{\lambda, \Lambda}^{+}(X-Y)+\widetilde{b}(x)|p-q|+\widetilde{\mu}|p-q|(|p|+|q|)+\widetilde{d}(x) \widetilde{\omega}(|r-s|) .
\end{aligned}
$$

The estimate from below in $(\widetilde{S C})^{\tilde{\mu}}$ is analogous. 
Notice that, with this definition and the choice of $\sigma$ in (3.12), we have

- $\|\widetilde{u}\|_{L^{\infty}\left(B_{2}\right)} \leq 1$ since $N \geq \sup _{B_{2}}|u(\sigma x)-u(0)|$;

- $\left\|\tilde{f}_{1}\right\|_{L^{p}\left(B_{2}\right)}=\frac{\sigma^{2-\frac{n}{p}}}{N}\|f\|_{L^{p}\left(B_{2 \sigma}\right)} \leq \sigma^{1-\frac{n}{p}} \frac{\|f\|_{L^{p}(\Omega)}}{W} \leq \frac{\delta}{16}$

- $\left\|\tilde{f}_{2}\right\|_{L^{p}\left(B_{2}\right)} \leq \frac{\sigma^{2-\frac{n}{p}}}{N} \omega(|u(0)|)\|d\|_{L^{p}\left(B_{2 \sigma}\right)} \leq \sigma^{1-\frac{n}{p}} \frac{\omega\left(\|u\|_{\infty}\right)\|d\|_{L^{p}(\Omega)}}{W} \leq \frac{\delta}{16}$; thus $\|\widetilde{f}\|_{L^{p}\left(B_{2}\right)} \leq \frac{\delta}{8}$

- $\widetilde{\mu}=N \mu \leq\left(1+2^{\beta} K_{1}\right) W_{0} \mu \sigma^{\beta} \leq \frac{\delta}{8 K^{2}\left|B_{1}\right|^{1 / p}}$

- $\|\widetilde{b}\|_{L^{p}\left(B_{2}\right)}=\sigma^{1-\frac{n}{p}}\|b\|_{L^{p}\left(B_{2 \sigma}\right)} \leq \frac{\delta}{16 K}$

- $\widetilde{\omega}(1)\|\widetilde{d}\|_{L^{p}\left(B_{2}\right)}=\sigma^{2-\frac{n}{p} \frac{\omega(N)}{N}}\|d\|_{L^{p}\left(B_{2 \sigma}\right)} \leq \sigma^{2-\frac{n}{p}} \omega(1)\|d\|_{L^{p}(\Omega)} \leq \frac{\delta}{32\left(K_{2}+K+1\right)}$ from the hypothesis $\omega(r) \leq \omega(1) r$ for all $r \geq 0$;

- $\left\|\bar{\beta}_{\widetilde{F}}(\cdot, 0)\right\|_{L^{p}\left(B_{1}\right)} \leq \delta / 4$, by choosing $\theta=\delta / 8$. Indeed,

$$
\begin{aligned}
\bar{\beta}_{\widetilde{F}}(x, & \left.x_{0}\right) \leq \frac{\sigma^{2}}{N} \sup _{X \in \mathbb{S}^{n}} \frac{\left|F\left(\sigma x, u(0), 0, \frac{N}{\sigma^{2}} X\right)-F\left(\sigma x, 0,0, \frac{N}{\sigma^{2}} X\right)\right|}{\|X\|+1} \\
& +\sup _{X \in \mathbb{S}^{n}} \frac{\left|F\left(\sigma x, 0,0, \frac{N}{\sigma^{2}} X\right)-F\left(\sigma x_{0}, 0,0, \frac{N}{\sigma^{2}} X\right)\right|}{\frac{N}{\sigma^{2}}(\|X\|+1)} \\
& +\frac{\sigma^{2}}{N} \sup _{X \in \mathbb{S}^{n}} \frac{\left|F\left(\sigma x_{0}, 0,0, \frac{N}{\sigma^{2}} X\right)-F\left(\sigma x_{0}, u(0), 0, \frac{N}{\sigma^{2}} X\right)\right|}{\|X\|+1} \\
& +\frac{\sigma^{2}}{N} \sup _{X \in \mathbb{S}^{n}} \frac{|F(\sigma x, u(0), 0,0)|+\left|F\left(\sigma x_{0}, u(0), 0,0\right)\right|}{\|X\|+1} \\
\leq & \frac{2 \sigma^{2}}{N}\left\{d(\sigma x)+d\left(\sigma x_{0}\right)\right\} \omega(|u(0)|) \sup _{X \in \mathbb{S}^{n}}(\|X\|+1)^{-1}+\beta_{F}\left(\sigma x, \sigma x_{0}\right)
\end{aligned}
$$

and therefore,

$$
\begin{aligned}
\left\|\bar{\beta}_{\widetilde{F}}(\cdot, 0)\right\|_{L^{p}\left(B_{1}\right)} & \leq 4 \sigma^{1-\frac{n}{p}} \frac{\omega\left(\|u\|_{L^{\infty}(\Omega)}\right)\|d\|_{L^{p}(\Omega)}}{W}+\left(\frac{1}{\sigma^{n}} \int_{B_{\sigma}(0)} \beta_{F}(y, 0)^{p} \mathrm{~d} y\right)^{\frac{1}{p}} \\
& \leq \delta / 8+\theta=\delta / 4 .
\end{aligned}
$$

Notice that the only place we had to use the dependence on the bound $C_{0}$ is to measure the smallness of $\mu$. Thus, if $\mu=0$, the final constant does not depend on $W_{0}$, neither on $C_{0}$.

Remark 3.10 Still for $\mu=0$, if we split our analysis in two cases (as usual for linear growth in the gradient, see for instance [27]), then we can obtain the conditions in terms of $\left(\bar{H}_{\theta}\right)$. Indeed, in this case, we consider $N:=W$. If $N \geq 1$, then $\widetilde{u}$ is as in claim 3.9. In this case, using $N / \sigma^{2} \geq 1$ we can replace $\beta$ by $\bar{\beta}$ in (3.14); for the estimate in $\widetilde{\omega}(1)\|\widetilde{d}\|_{L^{p}\left(B_{2}\right)}$ we only need $\omega(r) \leq \omega(1) r$ for $r \geq 1$. On the other hand, if $N \leq 1$, we just define $\widetilde{u}=u(\sigma x)$ and use that each of the addends in $W$ is less or equal than 1 and also 


$$
\widetilde{\omega}(1)\|\widetilde{d}\|_{L^{p}\left(B_{2}\right)}=\sigma^{2-\frac{n}{p}} \omega(N)\|d\|_{L^{p}\left(B_{2 \sigma}\right)} \leq \sigma^{2-\frac{n}{p}} \omega(1)\|d\|_{L^{p}(\Omega)} .
$$

Notice that, in this case, the final estimate we obtain for our original function $u$ is that $\|u\|_{C^{1, \alpha}(\Omega)} \leq C \leq C(W+1)$, instead of $\|u\|_{C^{1, \alpha}(\Omega)} \leq C W$.

We refer to remarks 6.4 and 6.5 in [58] concerning $\beta_{F}$ and $\bar{\beta}_{F}$; also theorem 7.3 there for an improvement of this estimate in the parabolic case in which $\|u\|_{C^{1, \alpha}(\Omega)}$ goes to zero when $W$ does.

In particular $\widetilde{F}, \widetilde{u}, \widetilde{\mu}, \widetilde{b}, \widetilde{d}, \widetilde{\omega}$ satisfy the hypotheses of lemma 3.8. Thus, if we show that $\|\widetilde{u}\|_{C^{1, \alpha}\left(\bar{B}_{1}\right)} \leq C$, we will obtain that

$$
\|u(\sigma x)-u(0)\|_{C^{1, \alpha}\left(\bar{B}_{1}\right)} \leq C N \leq\left(1+2^{\beta} K_{1}\right) C W
$$

by (3.13), then $\|u\|_{C^{1, \alpha}\left(\bar{B}_{\sigma}\right)} \leq C\left\{\|u\|_{L^{\infty}(\Omega)}+\|f\|_{L^{p}(\Omega)}\right\}$, where the constant depends on $\sigma$; the local estimate following by a covering argument.

Remark 3.11 In the case we have an arbitrary modulus of continuity, we define $N=\sigma \max \{W, 1\}+\sup _{x \in B_{2}}|u(\sigma x)-u(0)|$, which by construction and $C^{\beta}$ local superlinear estimate,

$$
\sigma \leq N \leq\left(\sigma+2^{\beta} K_{1} \sigma^{\beta}\right) \max \{W, 1\} \leq\left(1+2^{\beta} K_{1}\right) W_{0} \sigma^{\beta} \leq 1
$$

Then we have $\widetilde{\omega}(1)\|\widetilde{d}\|_{L^{p}\left(B_{2}\right)}=\frac{\sigma^{2-\frac{n}{p}}}{N} \omega(N)\|d\|_{L^{p}\left(B_{2 \sigma}\right)} \leq \sigma^{1-\frac{n}{p}} \omega(1)\|d\|_{L^{p}(\Omega)}$.

Moreover, we can consider the smallness assumption in terms of $(\bar{H})_{\theta}$, with $\bar{\beta}$ instead of $\beta$ in (3.14). In fact, in this case we use $N / \sigma^{2} \geq 1$. In the end, we obtain that the original function $u$ is such that $\|u\|_{C^{1, \alpha}(\bar{\Omega})}$ is bounded by $C \max \{W, 1\} \leq C(W+1)$, in place of $C W$.

With these rescalings in mind, we write $F, u, M, \mu, b, d, \omega$ instead of $\widetilde{F}, \widetilde{u}, \widetilde{\mu}, \widetilde{b}, \widetilde{d}, \widetilde{\omega}$, in order to ease of notation. Now we can proceed with Caffarelli's iterations as in [47], [26], [48], which consists of finding a sequence of linear functions $l_{k}(x):=a_{k}+b_{k} \cdot x$ such that

$$
\begin{aligned}
& (i)_{k} \quad\left\|u-l_{k}\right\|_{L^{\infty}\left(B_{r_{k}}\right)} \leq r_{k}^{1+\alpha} \\
& (i i)_{k}\left|a_{k}-a_{k-1}\right| \leq K_{2} r_{k-1}^{1+\alpha}, \quad\left|b_{k}-b_{k-1}\right| \leq K_{2} r_{k-1}^{\alpha} \\
& (i i i)_{k}\left|\left(u-l_{k}\right)\left(r_{k} x\right)-\left(u-l_{k}\right)\left(r_{k} y\right)\right| \leq\left(1+3 K_{1}\right) r_{k}^{1+\alpha}|x-y|^{\beta} \text { for } x, y \in B_{1},
\end{aligned}
$$

where $r_{k}=\gamma^{k}$ for some $\gamma \in(0,1)$, for all $k \geq 0$, with the convention $l_{-1} \equiv 0$.

Observe that this proves the result. Indeed, $b_{k}=b_{0}+\left(b_{1}-b_{0}\right)+\ldots+$ $\left(b_{k}-b_{k-1}\right)$ converges to some $b$, since $\sum_{k=0}^{\infty}\left|b_{k}-b_{k-1}\right| \leq K_{2} \sum_{k=0}^{\infty}\left(\gamma^{\alpha}\right)^{k-1}<\infty$; also $\left|b_{k}-b\right| \leq \sum_{l=k}^{\infty}\left|b_{l+1}-b_{l}\right| \leq K_{2} \sum_{l=k}^{\infty} \gamma^{\alpha l}=K_{2} \frac{\gamma^{\alpha k}}{1-\gamma^{\alpha}}$. Similarly, $\left|a_{k}-a\right| \leq$ $K_{2} \frac{\gamma^{k(1+\alpha)}}{1-\gamma^{1+\alpha}}$ and $a_{k}$ converges to some $a$. 
Next, for each $x \in B_{1}$, there exists $k \geq 0$ such that $r_{k+1}<|x| \leq r_{k}$. Then, $\left|u(x)-a_{k}-b_{k} \cdot x\right|=\left|u(x)-l_{k}(x)\right| \leq r_{k}^{1+\alpha}$, since $x \in \bar{B}_{r_{k}}$, thus

$$
\begin{aligned}
|u(x)-a-b \cdot x| & \leq\left|u(x)-a_{k}-b_{k} \cdot x\right|+\left|a_{k}-a\right|+\left|b_{k}-b\right||x| \\
& \leq r_{k}^{1+\alpha}+K_{2} \frac{r_{k}^{1+\alpha}}{1-\gamma^{1+\alpha}}+K_{2} \frac{r_{k}^{\alpha}}{1-\gamma^{\alpha}} r_{k} \\
& =\left\{1+\frac{K_{2}}{1-\gamma^{1+\alpha}}+\frac{K_{2}}{1-\gamma^{\alpha}}\right\} \frac{1}{\gamma^{1+\alpha}} r_{k+1}^{1+\alpha} \leq C_{\gamma}|x|^{1+\alpha}
\end{aligned}
$$

By definition of a differentiable function, $a=u(0), b=D u(0)$ and we will have obtained $|u(x)-u(0)-D u(0) \cdot x| \leq C|x|^{1+\alpha}$ and $|D u(0)| \leq C$.

Notice that there was nothing special in doing the initial argument around 0 , which we had supposed in the beginning of the proof, belonging to $\Omega^{\prime}$. Actually, by replacing it by any $x_{0} \in \Omega$ and setting the corresponding $s_{0}=\min \left\{r_{0}, \operatorname{dist}\left(x_{0}, \partial \Omega^{\prime}\right)\right\}$, we define $N=N_{\sigma}\left(x_{0}\right)$ by changing 0 by $x_{0}$ in there. Then, our initial function $u$ is differentiable at $x_{0}$ with

$$
\left|u(x)-u\left(x_{0}\right)-D u\left(x_{0}\right) \cdot\left(x-x_{0}\right)\right| \leq C W\left|x-x_{0}\right|^{1+\alpha}, \quad\left|D u\left(x_{0}\right)\right| \leq C W
$$

which implies, by appendix $\mathrm{C}$, that $D u \in C^{\alpha}\left(\bar{B}_{\sigma}\right)$ and $\|u\|_{C^{1, \alpha}\left(\bar{B}_{\sigma}\right)} \leq C W$. Thus, for the complete local estimate, we just take finitely many such points in order to cover $\Omega^{\prime}$.

We stress that $(i)_{k}$ and $(i i)_{k}$ are completely enough to imply the result, as above, while $(i i i)_{k}$ is an auxiliary tool to get them. So, let us prove $(i)_{k}-(i i i)_{k}$ by induction on $k$.

For $k=0$ we set $a_{0}=b_{0}=0$. Recall that $\beta$ and $K_{1}$ are the constants from the $C^{\beta}$ superlinear local estimate in $B_{1}$ such that $\|u\|_{C^{\beta}\left(\bar{B}_{1}\right)} \leq \widetilde{K}_{1}(1+\delta+1) \leq$ $3 K_{1}$, which implies $(i i i)_{0}$. Obviously $(i)_{0}$ and $(i i)_{0}$ are satisfied too.

Notice that $\left|b_{k}\right| \leq \sum_{l=0}^{k}\left|b_{l}-b_{l-1}\right| \leq \frac{K_{2}}{\gamma^{\alpha}} \sum_{k=0}^{\infty} \gamma^{\alpha k}=\frac{K_{2}}{\gamma^{\alpha}\left(1-\gamma^{\alpha}\right)} \leq K$ and also, for all $x \in B_{1},\left|l_{k}(x)\right| \leq\left|a_{k}\right|+\left|b_{k}\right||x| \leq \frac{K_{2}}{\gamma^{(1+\alpha)}} \sum_{k=0}^{\infty} \gamma^{(1+\alpha) k}+\frac{K_{2}}{\gamma^{\alpha}} \sum_{k=0}^{\infty} \gamma^{\alpha k}=K$.

As the induction step, we suppose the items $(i)_{k}-(i i i)_{k}$ valid in order to construct $a_{k+1}$ and $b_{k+1}$ for which $(i)_{k+1}-(i i i)_{k+1}$ hold. Define

$$
v(x)=v_{k}(x):=\frac{\left(u-l_{k}\right)\left(r_{k} x\right)}{r_{k}^{1+\alpha}}=\frac{u\left(r_{k} x\right)-a_{k}-b_{k} \cdot x r_{k}}{r_{k}^{1+\alpha}}, \text { for all } x \in B_{2} \text {. }
$$

Note that $(i)_{k}$ says precisely that $|v(x)| \leq 1$ for all $x \in B_{1}$. In addition, from 
this and $(i i i)_{k}$ we get

$$
\|v\|_{C^{\beta}\left(\bar{B}_{1}\right)}=\|v\|_{L^{\infty}\left(B_{1}\right)}+\sup _{\substack{x, y \in B_{1} \\ x \neq y}} \frac{|v(x)-v(y)|}{|x-y|^{\beta}} \leq 2+3 K_{1}=: K_{0} .
$$

Claim 3.12 $v$ is an $L^{p}$-viscosity solution of $F_{k}[v]=f_{k}(x)$ in $B_{2}$, for $f_{k}:=$ $f_{k}^{1}+f_{k}^{2}$ with $f_{k}^{1}(x):=r_{k}^{1-\alpha} f\left(r_{k} x\right) ; f_{k}^{2}(x):=-r_{k}^{1-\alpha} F\left(r_{k} x, l_{k}\left(r_{k} x\right), b_{k}, 0\right)$ and $F_{k}$ satisfying $(S C)_{F_{k}}^{\mu_{F_{k}}}$, where $F_{k}(x, s, p, X)$ is defined as

$$
r_{k}^{1-\alpha} F\left(r_{k} x, r_{k}^{1+\alpha} s+l_{k}\left(r_{k} x\right), r_{k}^{\alpha} p+b_{k}, r_{k}^{\alpha-1} X\right)-r_{k}^{1-\alpha} F\left(r_{k} x, l_{k}\left(r_{k} x\right), b_{k}, 0\right),
$$

and the coefficients as $b_{F_{k}}(x):=r_{k} b\left(r_{k} x\right)+2 r_{k} \mu K, \mu_{F_{k}}:=r_{k}^{1+\alpha} \mu, d_{F_{k}}(x):=$ $r_{k}^{2} d\left(r_{k} x\right)$ and $\omega_{F_{k}}(s):=r_{k}^{-1-\alpha} \omega\left(r_{k}^{1+\alpha} s\right)$.

Proof of Claim 3.12. Let $\varepsilon>0$ and $\psi \in W_{\text {loc }}^{2, p}\left(B_{2}\right)$ such that $v-\psi$ has a minimum (maximum) at $x_{0}$. Define $\varphi(x):=r_{k}^{1+\alpha} \psi\left(x / r_{k}\right)+l_{k}(x)$ for all $x \in B_{2 r_{k}}$; then $u-\psi$ has a minimum (maximum) at $r_{k} x_{0}$. Since $u$ is an $L^{p}$-viscosity solution in $B_{2 r_{k}}(0)$, there exists $r \in(0,2)$ such that

$$
F\left(r_{k} x, u\left(r_{k} x\right), D \varphi\left(r_{k} x\right), D^{2} \varphi\left(r_{k} x\right)\right) \leq(\geq) f\left(r_{k} x\right)+(-) r_{k}^{\alpha-1} \varepsilon \quad \text { a.e. in } B_{r}\left(x_{0}\right) .
$$

Using $D \psi(x)=r_{k}^{-\alpha}\left\{D \varphi\left(r_{k} x\right)-b_{k}\right\}$ and $D^{2} \psi(x)=r_{k}^{1-\alpha} D^{2} \varphi\left(r_{k} x\right)$ a.e., we get

$$
\begin{gathered}
r_{k}^{1-\alpha} F\left(r_{k} x, r_{k}^{1+\alpha} v(x)+l_{k}\left(r_{k} x\right), r_{k}^{\alpha} D \psi(x)+b_{k}, r_{k}^{\alpha-1} D^{2} \psi(x)\right) \\
\leq(\geq) r_{k}^{1-\alpha} f\left(r_{k} x\right)+(-) \varepsilon
\end{gathered}
$$

a.e. in $B_{r}\left(x_{0}\right)$. Adding $-r_{k}^{1-\alpha} F\left(r_{k} x, l_{k}\left(r_{k} x\right), b_{k}, 0\right)$ in both sides we obtain

$$
F_{k}\left(x, v(x), D \psi, D^{2} \psi\right) \leq(\geq) f_{k}(x)+(-) \varepsilon \quad \text { a.e. in } B_{r}\left(x_{0}\right)
$$

Moreover, $F_{k}$ satisfies $(S C)_{k}^{\mu_{F_{k}}}$, since $F_{k}(x, 0,0,0)=0$ a.e. $x \in B_{2}$ and

$$
\begin{aligned}
& F_{k}(x, r, p, X)-F_{k}(x, s, q, Y)=r_{k}^{1-\alpha}\left\{F\left(r_{k} x, r_{k}^{1+\alpha} r+l_{k}\left(r_{k} x\right), r_{k}^{\alpha} p+b_{k}, r_{k}^{\alpha-1} X\right)\right. \\
& \left.\quad-F\left(r_{k} x, r_{k}^{1+\alpha} s+l_{k}\left(r_{k} x\right), r_{k}^{\alpha} q+b_{k}, r_{k}^{\alpha-1} Y\right)\right\} \\
& \leq \mathcal{M}_{\lambda, \Lambda}^{+}(X-Y)+r_{k} b\left(r_{k} x\right)|p-q| \\
& \quad+r_{k} \mu|p-q|\left\{r_{k}^{\alpha}(|p|+|q|)+b_{k}\right\}+r_{k}^{1-\alpha} d\left(r_{k} x\right) \omega\left(r_{k}^{1+\alpha}|r-s|\right) \\
& =\mathcal{M}_{\lambda, \Lambda}^{+}(X-Y)+b_{F_{k}}(x)|p-q|+\mu_{F_{k}}|p-q|(|p|+|q|)+d_{F_{k}}(x) \omega_{F_{k}}(|r-s|)
\end{aligned}
$$

and the left hand side is completely analogous. 
Note that $F_{k}, v, \mu_{F_{k}}, b_{F_{k}}, d_{F_{k}}, \omega_{F_{k}}$ also satisfy the hypotheses of lemma 3.8,

$$
\begin{aligned}
& \left\|b_{F_{k}}\right\|_{L^{p}\left(B_{1}\right)} \leq r_{k}^{1-\frac{n}{p}}\|b\|_{L^{p}\left(B_{r_{k}}\right)}+2 \mu K\left|B_{1}\right|^{1 / p} \leq \delta \\
& \left\|f_{k}^{1}\right\|_{L^{p}\left(B_{1}\right)} \leq r_{k}^{1-\frac{n}{p}-\alpha}\|f\|_{L^{p}\left(B_{r_{k}}\right)} \leq \frac{\delta}{2} \\
& \left\|f_{k}^{2}\right\|_{L^{p}\left(B_{1}\right)} \leq r_{k}^{1-\frac{n}{p}-\alpha}\left\{\|b\|_{L^{p}\left(B_{r_{k}}\right)}\left|b_{k}\right|+(K+1) \omega(1)\|d\|_{L^{p}\left(B_{r_{k}}\right)}\right\} \\
& \quad+r_{k}^{1-\alpha} \mu\left|b_{k}\right|^{2}\left|B_{1}\right|^{\frac{1}{p}} \leq \frac{\delta}{2} \\
& \quad \\
& \omega_{F_{k}}(1)\left\|d_{F_{k}}\right\|_{L^{p}\left(B_{1}\right)}=r_{k}^{1-\frac{n}{p}-\alpha} \omega\left(r_{k}^{1+\alpha}\right)\|d\|_{L^{p}\left(B_{r_{k}}\right)} \leq r_{k}^{1-\frac{n}{p}-\alpha} \omega(1)\|d\|_{L^{p}\left(B_{1}\right)} \leq \delta
\end{aligned}
$$

and

$$
\begin{aligned}
\bar{\beta}_{F_{k}}\left(x, x_{0}\right) \leq & r_{k}^{1-\alpha} \sup _{X \in \mathbb{S}^{n}} \frac{\left|F\left(r_{k} x, l_{k}\left(r_{k} x\right), b_{k}, r_{k}^{\alpha-1} X\right)-F\left(r_{k} x, 0,0, r_{k}^{\alpha-1} X\right)\right|}{\|X\|+1} \\
& +\sup _{X \in \mathbb{S}^{n}} \frac{\left|F\left(r_{k} x, 0,0, r_{k}^{\alpha-1} X\right)-F\left(r_{k} x_{0}, 0,0, r_{k}^{\alpha-1} X\right)\right|}{r_{k}^{\alpha-1}(\|X\|+1)} \\
& +r_{k}^{1-\alpha} \sup _{X \in \mathbb{S}^{n}} \frac{\left|F\left(r_{k} x_{0}, 0,0, r_{k}^{\alpha-1} X\right)-F\left(r_{k} x_{0}, l_{k}\left(r_{k} x_{0}\right), b_{k}, r_{k}^{\alpha-1} X\right)\right|}{\|X\|+1} \\
& +r_{k}^{1-\alpha} \sup _{X \in \mathbb{S}^{n}} \frac{\left|F\left(r_{k} x, l_{k}\left(r_{k} x\right), b_{k}, 0\right)\right|+\left|F\left(r_{k} x_{0}, l_{k}\left(r_{k} x_{0}\right), b_{k}, 0\right)\right|}{\|X\|+1} \\
\leq & 2 r_{k}^{1-\alpha}\left\{\left(d\left(r_{k} x\right)+d\left(r_{k} x_{0}\right)\right) \omega\left(\left\|l_{k}\left(r_{k} x\right)\right\|_{L^{\infty}(\Omega)}\right)+\left(b\left(r_{k} x\right)+b\left(r_{k} x_{0}\right)\right)\right. \\
& \left.\left|b_{k}\right|+\mu\left|b_{k}\right|^{2}\right\} \sup _{X \in \mathbb{S}^{n}}(\|X\|+1)^{-1}+\bar{\beta}_{F}\left(r_{k} x, r_{k} x_{0}\right)
\end{aligned}
$$

since $r_{k}^{\alpha-1} \geq 1$, then

$$
\begin{aligned}
\left\|\bar{\beta}_{F_{k}}(\cdot, 0)\right\|_{L^{p}\left(B_{1}\right)} & \leq 4 r_{k}^{1-\frac{n}{p}-\alpha}(K+1) \omega(1)\|d\|_{L^{p}\left(B_{r_{k}}\right)}+4 K r_{k}^{1-\frac{n}{p}-\alpha}\|b\|_{L^{p}\left(B_{r_{k}}\right)} \\
& +2 \mu K^{2}\left|B_{1}\right|^{1 / p}+\left\|\bar{\beta}_{F}(\cdot, 0)\right\|_{L^{p}\left(B_{r_{k}}\right)} \leq \delta
\end{aligned}
$$

Let $h=h_{k} \in C\left(\bar{B}_{1}\right)$ be the $C$-viscosity solution of

$$
\left\{\begin{aligned}
F_{k}\left(0,0,0, D^{2} h\right) & =0 \text { in } B_{1} \\
h & =v \text { on } \partial B_{1} .
\end{aligned}\right.
$$

By ABP we have $\|h\|_{L^{\infty}\left(B_{1}\right)} \leq\|h\|_{L^{\infty}\left(\partial B_{1}\right)} \leq 1$ and by the $C^{1, \bar{\alpha}}$ local estimate (proposition 2.22), $\|h\|_{C^{1, \bar{\alpha}}\left(\bar{B}_{1 / 2}\right)} \leq K_{2}\|h\|_{L^{\infty}\left(B_{1}\right)} \leq K_{2}$. Hence, by lemma 3.8 applied to $F_{k}, v, \mu_{F_{k}}, b_{F_{k}}, d_{F_{k}}, \omega_{F_{k}}, \psi:=\left.v\right|_{\partial B_{1}}, \tau:=\beta, K_{0}$ and $h$ we obtain, for $\varepsilon$ given in (3.10), that $\|v-h\|_{L^{\infty}\left(B_{1}\right)} \leq \varepsilon$.

Define $\bar{l}(x)=\bar{l}_{k}(x):=h(0)+D h(0) \cdot x$ in $B_{1}$, then,

$$
\|v-\bar{l}\|_{L^{\infty}\left(B_{2 \gamma}\right)} \leq \gamma^{1+\alpha} .
$$


In fact, by the choice of $\gamma \leq \frac{1}{4}$ in (3.9), we have for all $x \in B_{2 \gamma}(0)$ that

$$
\begin{aligned}
|v(x)-\bar{l}(x)| & \leq|v(x)-h(x)|+|h(x)-h(0)-D h(0) \cdot x| \\
& \leq K_{2}(2 \gamma)^{1+\bar{\alpha}}+K_{2}|x|^{1+\bar{\alpha}} \leq 2 K_{2}(2 \gamma)^{1+\bar{\alpha}} \leq \gamma^{1+\alpha} .
\end{aligned}
$$

However, inequality (3.15) and the definition of $v$ imply

$\left|u\left(r_{k} x\right)-l_{k}\left(r_{k} x\right)-r_{k}^{1+\alpha} h(0)-r_{k}^{1+\alpha} D h(0) \cdot x\right| \leq r_{k}^{1+\alpha} \gamma^{1+\alpha}=r_{k+1}^{1+\alpha}$ for $x \in B_{2 \gamma}$,

which is equivalent to

$$
\left|u(y)-l_{k+1}(y)\right| \leq r_{k}^{1+\alpha} \gamma^{1+\alpha}=r_{k+1}^{1+\alpha} \text { for all } y=r_{k} x \in B_{2 \gamma r_{k}}=B_{2 r_{k+1}}
$$

where $l_{k+1}(y):=l_{k}(y)+r_{k}^{1+\alpha} h(0)+r_{k}^{\alpha} D h(0) \cdot y$. Then, we define

$$
a_{k+1}:=a_{k}+h(0) r_{k}^{1+\alpha}, \quad b_{k+1}:=b_{k}+D h(0) r_{k}^{\alpha}
$$

obtaining $(i)_{k+1}$. Further, $\left|a_{k+1}-a_{k}\right| \leq K_{2} r_{k}^{1+\alpha},\left|b_{k+1}-b_{k}\right| \leq K_{2} r_{k}^{\alpha}$, which is $(i i)_{k+1}$. To finish we note that, in order to prove $(i i i)_{k+1}$, it is enough to show

$$
\|v-\bar{l}\|_{C^{\beta}\left(\bar{B}_{\gamma}\right)} \leq\left(1+2 K_{1}\right) \gamma^{1+\alpha-\beta} .
$$

Indeed, if $x, y \in B_{1}$ and (3.16) is true, then

$$
\begin{aligned}
& |(v-\bar{l})(\gamma x)-(v-\bar{l})(\gamma y)| \leq\left(1+2 K_{1}\right) \gamma^{1+\alpha-\beta}|\gamma x-\gamma y|^{\beta} \\
& \Leftrightarrow\left|\left(u-l_{k}\right)\left(\gamma r_{k} x\right)-\left(u-l_{k}\right)\left(\gamma r_{k} y\right)-r_{k}^{\alpha} \operatorname{Dh}(0) \cdot(x-y) \gamma r_{k}\right| \\
& \quad \leq\left(1+2 K_{1}\right) \gamma^{1+\alpha} r_{k}^{1+\alpha}|x-y|^{\beta} \\
& \Leftrightarrow\left|\left(u-l_{k+1}\right)\left(r_{k+1} x\right)-\left(u-l_{k+1}\right)\left(r_{k+1} y\right)\right| \leq\left(1+2 K_{1}\right) r_{k+1}^{1+\alpha}|x-y|^{\beta} .
\end{aligned}
$$

Now, we obtain (3.16) applying the local quadratic $C^{\beta}$ estimate (proposition 2.14) to the function $w:=v-\bar{l}$, which is an $L^{p}$-viscosity solution in $B_{2}$ of the inequalities

$$
\mathcal{L}_{k}^{-}[w]-\mu_{F_{k}}|D w|^{2} \leq g_{k}(x), \quad \mathcal{L}_{k}^{+}[w]+\mu_{F_{k}}|D w|^{2} \geq-g_{k}(x)
$$

where $g_{k}:=g_{k}^{1}+g_{k}^{2}$, for $g_{k}^{1}(x):=\left|f_{k}(x)-F_{k}(x, \bar{l}(x), D h(0), 0)\right|$ and $g_{k}^{2}(x):=$ $d_{F_{k}}(x) \omega_{F_{k}}(|w|)$, with $\mathcal{L}_{k}^{ \pm}[u]:=\mathcal{M}_{\lambda, \Lambda}^{ \pm}\left(D^{2} u\right) \pm\left(b_{F_{k}}+2 K_{2} \mu_{F_{k}}\right)|D u|$. Surely, this finishes the proof of (3.16), since

$$
\left|g_{k}^{1}(x)\right| \leq\left|f_{k}(x)\right|+b_{F_{k}}(x)|D h(0)|+\omega_{F_{k}}(|\bar{l}(x)|) d_{F_{k}}(x)+\mu_{F_{k}}|D h(0)|^{2}
$$


then using that $|\bar{l}(x)| \leq|h(0)|+|D h(0)||x| \leq\|h\|_{C^{1, \bar{\alpha}}\left(\bar{B}_{1 / 2}\right)} \leq K_{2}$ for all $x \in B_{1}$, we have

$$
\begin{aligned}
& \left\|g_{k}\right\|_{L^{p}\left(B_{1}\right)} \leq\left\|f_{k}\right\|_{L^{p}\left(B_{1}\right)}+\left\|b_{F_{k}}\right\|_{L^{p}\left(B_{1}\right)} K_{2}+\left(K_{2}+1\right) \omega_{F_{k}}(1)\left\|d_{F_{k}}\right\|_{L^{p}\left(B_{1}\right)} \\
& \quad+\mu K_{2}^{2}\left|B_{1}\right|^{\frac{1}{p}}+\left(1+\|w\|_{L^{\infty}\left(B_{1}\right)}\right) \omega_{F_{k}}(1)\left\|d_{F_{k}}\right\|_{L^{p}\left(B_{1}\right)} \leq\left(5+2 K_{2}\right) \delta \leq \gamma^{\alpha}
\end{aligned}
$$

from the definition of $\delta$ in (3.11). Thus, using the estimate above and (3.15) in the $C^{\beta}$ local estimate, properly scaled to the ball of radius $\gamma$, we obtain in particular that

$$
\begin{aligned}
{[w]_{\beta, \overline{B_{\gamma}}} } & \leq \gamma^{-\beta} \widetilde{K}_{1}\left\{\|w\|_{L^{\infty}\left(B_{2 \gamma}\right)}+\gamma^{2-\frac{n}{p}}\left\|g_{k}\right\|_{L^{p}\left(B_{2 \gamma}\right)}\right\} \\
& \leq \gamma^{-\beta} K_{1}\left\{\gamma^{1+\alpha}+\gamma^{2-\frac{n}{p}} \gamma^{\alpha}\right\} \leq 2 K_{1} \gamma^{1+\alpha-\beta}
\end{aligned}
$$

and so

$$
\begin{aligned}
\|w\|_{C^{\beta}\left(\overline{B_{\gamma}}\right)} & =\|w\|_{L^{\infty}\left(B_{\gamma}\right)}+[w]_{\beta, \overline{B_{\gamma}}} \\
& \leq \gamma^{1+\alpha}+2 K_{1} \gamma^{1+\alpha-\beta} \leq\left(1+2 K_{1}\right) \gamma^{1+\alpha-\beta}
\end{aligned}
$$

which is (3.16), as desired.

Remark 3.13 By the proof above we see that, under $\mu,\|b\|_{L^{p}(\Omega)}, \omega(1)\|d\|_{L^{p}(\Omega)}$ $\leq C_{1}$, both $\sigma$ and the final constant $C$ depend on $n, p, \lambda, \Lambda, \alpha, \beta, K_{1}, K_{2}, C_{0}$ and $C_{1}$. This is very useful in applications, when we have, for example, a sequence of solutions $u_{k}$ with their respective coefficients uniformly bounded; with $\left\|u_{k}\right\|_{L^{\infty}}$ and the $L^{p}$ norm of the right hand side a priori bounded. Then we can uniformly bound the $C^{1, \alpha}$ norm of $u_{k}$.

\subsection{2}

\section{Boundary Regularity}

Since our equation is invariant under diffeomorphisms and $\partial \Omega \in C^{1,1}$, we only need to prove regularity and estimates for some half ball, say $B_{1}^{+}(0)$. Precisely, we need to prove the following theorem.

Theorem 3.14 Suppose $F$ satisfies $(S C)^{\mu}, f \in L^{p}\left(B_{1}^{+}\right)$, for $p>n$ and $\psi \in C^{1, \tau}(\mathbb{T})$. Let $u \in C(\bar{\Omega})$ be an $L^{p}$-viscosity solution of

$$
\left\{\begin{array}{rlrl}
F\left(x, u, D u, D^{2} u\right) & =f(x) \text { in } B_{1}^{+} \\
u & =\psi & \text { on } & \mathbb{T}_{1}
\end{array}\right.
$$

with $\|u\|_{L^{\infty}\left(B_{1}^{+}\right)}+\|f\|_{L^{p}\left(B_{1}^{+}\right)}+\|\psi\|_{C^{1, \tau}\left(\mathbb{T}_{1}\right)} \leq C_{0}$. Let $\alpha \in(0, \bar{\alpha})$ such that $\alpha \leq \min (\beta, 1-n / p, \tau, \bar{\alpha}(1-\tau))$. Then, there exists $\theta=\theta\left(n, p, \lambda, \Lambda, \alpha, \bar{\alpha}, K_{3}\right)$ 
such that if $\left(H_{\theta}\right)$ holds for some $r_{0}>0$ and for all $x_{0} \in \bar{B}_{1}^{+}$, this implies that $u \in C^{1, \alpha}\left(\overline{B_{1 / 2}^{+}}\right)$and

$$
\|u\|_{C^{1, \alpha}\left(\overline{B_{1 / 2}^{+}}\right)} \leq C\left\{\|u\|_{L^{\infty}\left(B_{1}^{+}\right)}+\|f\|_{L^{p}\left(B_{1}^{+}\right)}+\|\psi\|_{C^{1, \tau}\left(\mathbb{T}_{1}\right)}\right\}
$$

where $C$ depends on $r_{0}, n, p, \lambda, \Lambda, \alpha, \bar{\alpha}, \beta, \mu,\|b\|_{L^{p}\left(B_{1}^{+}\right)}, \omega(1)\|d\|_{L^{p}\left(B_{1}^{+}\right)}, K_{1}, K_{3}$ and the bound $C_{0}$.

Here, $K_{1}, \beta$ is the pair of $C^{\beta}$ global superlinear estimate (proposition 2.14) in $B_{1}^{+}$, related to the initial $n, p, \lambda, \Lambda, \mu,\|b\|_{L^{p}(\Omega)}, \tau$ and $C_{1}$, such that

$$
\|u\|_{C^{\beta}\left(\bar{B}_{1}^{+}\right)} \leq K_{1}\left\{\|u\|_{L^{\infty}\left(B_{1}^{+}\right)}+\|f\|_{L^{p}\left(B_{1}^{+}\right)}+\|\psi\|_{C^{\tau}\left(\mathbb{T}_{1}\right)}+\|d\|_{L^{p}\left(B_{1}^{+}\right)} \omega\left(\|u\|_{L^{\infty}\left(B_{1}^{+}\right)}\right)\right\} .
$$

As in [38], we start proving a boundary version of the approximation lemma in $B_{1}^{\nu}$. For this set, let $K_{3} \geq 1$ and $\bar{\alpha}$ be the pair of $C^{1, \bar{\alpha}}$ boundary estimate (proposition 2.24) associated to $n, \lambda, \Lambda$ and $\tau$, independently of $\nu>0$.

We can suppose that $K_{1} \geq \widetilde{K}_{1}$ and $\beta \leq \widetilde{\beta}$, where $\widetilde{K}_{1}, \widetilde{\beta}$ is the pair of $C^{\beta}$ global estimate for the set $B_{1}^{\nu}$ (or $B_{1 / 2}^{\nu}$ ), independently of $\nu>0$, with respect to an equation with given constants $n, p, \lambda, \Lambda$ and bounds for the coefficients $\mu \leq 1,\|b\|_{L^{p}\left(B_{2}^{\nu}\right)} \leq 1+2 K_{3}\left(3+2 C_{n}\right)\left|B_{1}\right|^{1 / p}$ (for a constant $C_{n}$, depending only on $n$, from lemma 6.35 of [41] for $\epsilon=1 / 2$, that will appear in the sequel) and $\omega(1)\|d\|_{L^{p}\left(B_{2}^{\nu}\right)} \leq 1$, for any solution in $B_{2}^{\nu}$ satisfying $\|u\|_{L^{\infty}\left(B_{2}^{\nu}\right)} \leq 1$ and $\|\psi\|_{C^{1, \tau}\left(\mathbb{T}_{2}^{\nu}\right)} \leq 2$ (or for any solution in $B_{1}^{\nu}$ with coefficients in $B_{1}^{\nu}$ ).

Lemma 3.15 Assume $F$ satisfies $(S C)^{\mu}$ in $B_{1}^{\nu}$ for some $\nu \in[0,1]$ and $f \in L^{p}\left(B_{1}^{\nu}\right)$, where $p>n$. Let $\psi \in C^{\tau}\left(\partial B_{1}^{\nu}\right)$ with $\|\psi\|_{C^{\tau}\left(\partial B_{1}^{\nu}\right)} \leq K_{0}$. Then, for all $\varepsilon>0$, there exists $\delta \in(0,1), \delta=\delta\left(\varepsilon, n, p, \lambda, \Lambda, \tau, K_{0}\right)$, such that if

$$
\left\|\bar{\beta}_{F}(\cdot, 0)\right\|_{L^{p}\left(B_{1}^{\nu}\right)} \leq \delta,\|f\|_{L^{p}\left(B_{1}^{\nu}\right)} \leq \delta, \mu \leq \delta,\|b\|_{L^{p}\left(B_{1}^{\nu}\right)} \leq \delta, \omega(1)\|d\|_{L^{p}\left(B_{1}^{\nu}\right)} \leq \delta
$$

then any two $L^{p}$-viscosity solutions $v$ and $h$ of

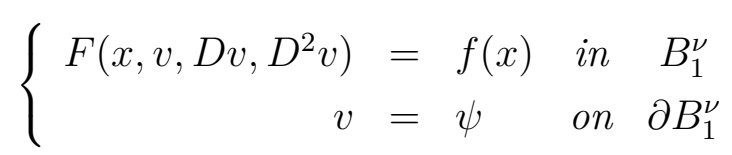

and

$$
\left\{\begin{aligned}
F\left(0,0,0, D^{2} h\right) & =0 \text { in } B_{1}^{\nu} \\
h & =\psi \text { on } \partial B_{1}^{\nu}
\end{aligned}\right.
$$

respectively, satisfy $\|v-h\|_{L^{\infty}\left(B_{1}^{\nu}\right)} \leq \varepsilon$. 
Proof. For $\varepsilon>0$, we will prove the existence of $\delta \in(0,1)$ as above with $\delta \leq 2^{-\frac{n}{2 p}} \widetilde{\delta}^{1 / 2}$, where $\widetilde{\delta}$ is the constant from proposition 2.6. Suppose the contrary, then there exist $\varepsilon_{0}>0$ and sequences $\nu_{k} \in[0,1], F_{k}$ satisfying $(S C)^{\mu_{k}}$ for $b_{k}, d_{k} \in L_{+}^{p}\left(B_{1}^{\nu_{k}}\right), \mu_{k} \geq 0, \omega_{k}$ modulus, $\delta_{k} \in(0,1)$ with $\delta_{k} \leq 2^{-\frac{n}{2 p}} \widetilde{\delta}_{k}^{1 / 2}$ and $f_{k} \in L^{p}\left(B_{1}^{\nu_{k}}\right)$ such that

$$
\left\|\bar{\beta}_{F_{k}}(\cdot, 0)\right\|_{L^{p}\left(B_{1}^{\nu_{k}}\right)},\left\|f_{k}\right\|_{L^{p}\left(B_{1}^{\nu_{k}}\right)}, \mu_{k},\left\|b_{k}\right\|_{L^{p}\left(B_{1}^{\nu_{k}}\right)}, \omega_{k}(1)\left\|d_{k}\right\|_{L^{p}\left(B_{1}^{\nu_{k}}\right)} \leq \delta_{k} \underset{k \rightarrow \infty}{\longrightarrow} 0
$$

with $v_{k}, h_{k} \in C\left(\overline{B_{1}^{\nu k}}\right) L^{p}$-viscosity solutions of

$$
\left\{\begin{aligned}
F_{k}\left(x, v_{k}, D v_{k}, D^{2} v_{k}\right) & =f_{k}(x) & \text { in } B_{1}^{\nu_{k}} \\
v_{k} & =\psi_{k} & \text { on } \partial B_{1}^{\nu_{k}}
\end{aligned}\right.
$$

and

$$
\left\{\begin{aligned}
F_{k}\left(0,0,0, D^{2} h_{k}\right) & =0 \quad \text { in } \quad B_{1}^{\nu_{k}} \\
h_{k} & =\psi_{k} \text { on } \partial B_{1}^{\nu_{k}}
\end{aligned}\right.
$$

where $\left\|\psi_{k}\right\|_{C^{\tau}\left(\partial B_{1}^{\nu_{k}}\right)} \leq K_{0}$ but $\left\|v_{k}-h_{k}\right\|_{L^{\infty}\left(B_{1}^{\left.\nu_{k}\right)}\right.}>\varepsilon_{0}$.

Analogously to the proof of lemma 3.8, ABP implies that $\left\|v_{k}\right\|_{L^{\infty}\left(B_{1}^{\nu_{k}}\right)},\left\|h_{k}\right\|_{L^{\infty}\left(B_{1}^{\nu_{k}}\right)} \leq C_{0}$ for large $k$, where $C_{0}$ is a constant that depends only on $n, p, \lambda, \Lambda$ and $K_{0}$.

Notice that $B_{1}^{\nu_{k}}$ has the exterior cone property, then by the $C^{\beta}$ global quadratic estimate (proposition 2.14) we obtain $\beta \in(0,1)$ such that

$$
\left\|v_{k}\right\|_{C^{\beta}\left(\overline{B_{1}^{\nu k}}\right)},\left\|h_{k}\right\|_{C^{\beta}\left(\overline{B_{1}^{\nu k}}\right)} \leq C, \quad \text { for all } k \in \mathbb{N}
$$

where $\beta=\min \left(\beta_{0}, \tau / 2\right)$ for some $\beta_{0}=\beta_{0}(n, p, \lambda, \Lambda)$ and $C$ depending on $n, p, \lambda, \Lambda$ and $C_{0}$. Observe that $\beta$ and $C$ do not depend on $k$, since $\mu_{k}$, $\left\|b_{k}\right\|_{L^{p}\left(B_{1}^{\nu_{k}}\right)}, \omega_{k}(1)\left\|d_{k}\right\|_{L^{p}\left(B_{1}^{\nu_{k}}\right)},\left\|f_{k}\right\|_{L^{p}\left(B_{1}^{\nu_{k}}\right)} \leq 1$ and $\operatorname{diam}\left(B_{1}^{\nu_{k}}\right) \leq 2$, for all $k \in \mathbb{N}$. Here we have different domains, what prevents us from directly using the compact inclusion $C^{\beta}$ into the set of continuous functions, in order to produce convergent subsequences. But this is just a technicality, as in [38], by taking a subsequence of $\nu_{k}$ that converges to some $\nu_{\infty} \in[0,1]$, which we can suppose monotonous. Hence we consider two cases: $B_{1}^{\nu_{\infty}} \subset B_{1}^{\nu_{k}} \subset B_{1}^{\nu_{k+1}} \subset \ldots$ or $\ldots \subset B_{1}^{\nu_{k+1}} \subset B_{1}^{\nu_{k}} \subset B_{1}^{\nu_{\infty}}$, for all $k \in \mathbb{N}$. In the first one, we use the compact inclusion on $\overline{B_{1}^{\nu \infty}}$. In the second, we make a trivial extension of our functions to the larger domain $\overline{B_{1}^{\nu \infty}}$, i.e. by defining $\psi_{k}$ in $\widetilde{B}_{k}=B_{1} \cap\left\{-\nu_{\infty} \leq x_{n} \leq-\nu_{k}\right\}$ in such a way that $\left\|\psi_{k}\right\|_{C^{\tau}\left(\widetilde{B}_{k}\right)} \leq C_{0}$, from where we can suppose that (3.18) holds on $\overline{B_{1}^{\nu \infty}}$ for the extended $v_{k}$ and $h_{k}$. In both cases, we obtain convergent subsequences $v_{k} \longrightarrow v_{\infty}, h_{k} \longrightarrow h_{\infty}$ in $C\left(\overline{B_{1}^{\nu \infty}}\right)$ as $k \rightarrow \infty$, for some continuous 
functions $v_{\infty}, h_{\infty}$ in $\overline{B_{1}^{\nu_{\infty}}}$, with $v_{\infty}=h_{\infty}=\psi_{\infty}$ on $\partial B_{1}^{\nu_{\infty}}$.

Finally, we claim that $v_{\infty}$ and $h_{\infty}$ are viscosity solutions of

$$
\left\{\begin{array}{rllc}
F_{\infty}\left(D^{2} u\right) & =0 & \text { in } & B_{1}^{\nu_{\infty}} \\
u & =\psi_{\infty} & \text { on } & \partial B_{1}^{\nu_{\infty}}
\end{array},\right.
$$

therefore equal by proposition 2.23 , which contradicts $\left\|v_{\infty}-h_{\infty}\right\|_{L^{\infty}\left(B_{1}\right)} \geq \varepsilon_{0}$.

For $h_{\infty}$, it follows by passing to uniform limits in the inequalities satisfied by $h_{k}$. For $v_{\infty}$, we apply proposition 2.12 together with observation 2.13 , since for each $\varphi \in C^{2}(B)$, where $B \subset B_{1}^{\nu \infty}$, we have that $F_{k}\left(x, v_{k}, D \varphi, D^{2} \varphi\right)-$ $f_{k}(x)-F_{\infty}\left(D^{2} \varphi\right) \rightarrow 0$ as $k \rightarrow \infty$ in $L^{p}(B)$, analogously to the end of the proof of lemma 3.8 .

Proof of theorem 3.14. We proceed as in the local case, introducing the corresponding changes that come from the boundary context. Our approach is similar to [38]. Now we set $W:=\|u\|_{L^{\infty}\left(B_{1}^{+}\right)}+\|f\|_{L^{p}\left(B_{1}^{+}\right)}+\|\psi\|_{C^{1, \tau}\left(\mathbb{T}_{1}\right)}+$ $\|d\|_{L^{p}\left(B_{1}^{+}\right)} \omega\left(\|u\|_{L^{\infty}\left(B_{1}^{+}\right)}\right) \leq W_{0}$ and $s_{0}:=\min \left(r_{0}, \frac{1}{2}\right)$.

Fix $\alpha \in(0, \bar{\alpha})$ with $\alpha \leq \min \left(\beta, 1-\frac{n}{p}, \tau, \bar{\alpha}(1-\tau)\right)$ and choose $\gamma=$ $\gamma\left(n, \alpha, \bar{\alpha}, K_{3}\right) \in\left(0, \frac{1}{4}\right]$ such that $2^{2+\bar{\alpha}} K_{4} \gamma^{\bar{\alpha}} \leq \gamma^{\alpha}$, where $K_{4}=K_{4}\left(K_{3}, n\right) \geq 1$ will be specified later. Thus, define $\varepsilon=\varepsilon(\gamma)$ by $K_{4}(2 \gamma)^{1+\bar{\alpha}}$. This $\varepsilon$ provides a $\delta=\delta(\varepsilon) \in(0,1)$, the constant of the approximation lemma 3.15 which, up to diminishing, can be supposed to satisfy $\left(5+2 K_{4}\right) \delta \leq \gamma^{\alpha}$. Next we chose $\sigma=\sigma\left(s_{0}, n, p, \alpha, \bar{\alpha}, \beta, \delta, \mu,\|b\|_{L^{p}\left(B_{1}^{+}\right)}, \omega(1)\|d\|_{L^{p}\left(B_{1}^{+}\right)}, K_{1}, K_{3}, C_{0}\right) \leq \frac{s_{0}}{2}$ such that

$$
\sigma^{\min \left(1-\frac{n}{p}, \beta\right)} m \leq \delta\left\{32 K^{2}\left(K_{4}+K+1\right)\left|B_{1}\right|^{1 / p}\right\}^{-1}
$$

where $m:=\max \left\{1,\|b\|_{L^{p}\left(B_{1}^{+}\right)}, \omega(1)\|d\|_{L^{p}\left(B_{1}^{+}\right)}, \mu\left(1+2^{\beta} K_{1}\right) W_{0}\right\}$ and $K:=$ $K_{4} \gamma^{-\alpha}\left(1-\gamma^{\alpha}\right)^{-1}+K_{4} \gamma^{-1-\alpha}\left(1-\gamma^{1+\alpha}\right)^{-1} \geq K_{4} \geq 1$.

Fix $z=\left(z^{\prime}, z_{n}\right) \in B_{1 / 2}^{+}(0)$. We split our analysis in two cases, depending on the distance of the point $z$ to the bottom boundary:

$$
\text { 1) } z_{n}<\frac{\sigma}{2} \Leftrightarrow \nu<\frac{1}{2} \text { and 2) } z_{n} \geq \frac{\sigma}{2} \Leftrightarrow \nu \geq \frac{1}{2} \text {, for } \nu:=\frac{z_{n}}{\sigma} \text {. }
$$

Suppose the first one. In this case we proceed as in [38] by translating the problem to the set $B_{2}^{\nu}$, in order to use the approximation lemma in its boundary version 3.15. Notice that, for $B_{2 \sigma}^{+}(z)=B_{2 \sigma}(z) \cap\left\{x_{n}>0\right\}$, which is a subset of $B_{1}^{+}(0)$, and for $B_{2}^{\nu}(0)=B_{2}(0) \cap\left\{x_{n}>-\nu\right\}$, we have

$$
x \in B_{2}^{\nu}(0) \Leftrightarrow \sigma x+z \in B_{2 \sigma}^{+}(z) \subset B_{1}^{+}(0) .
$$



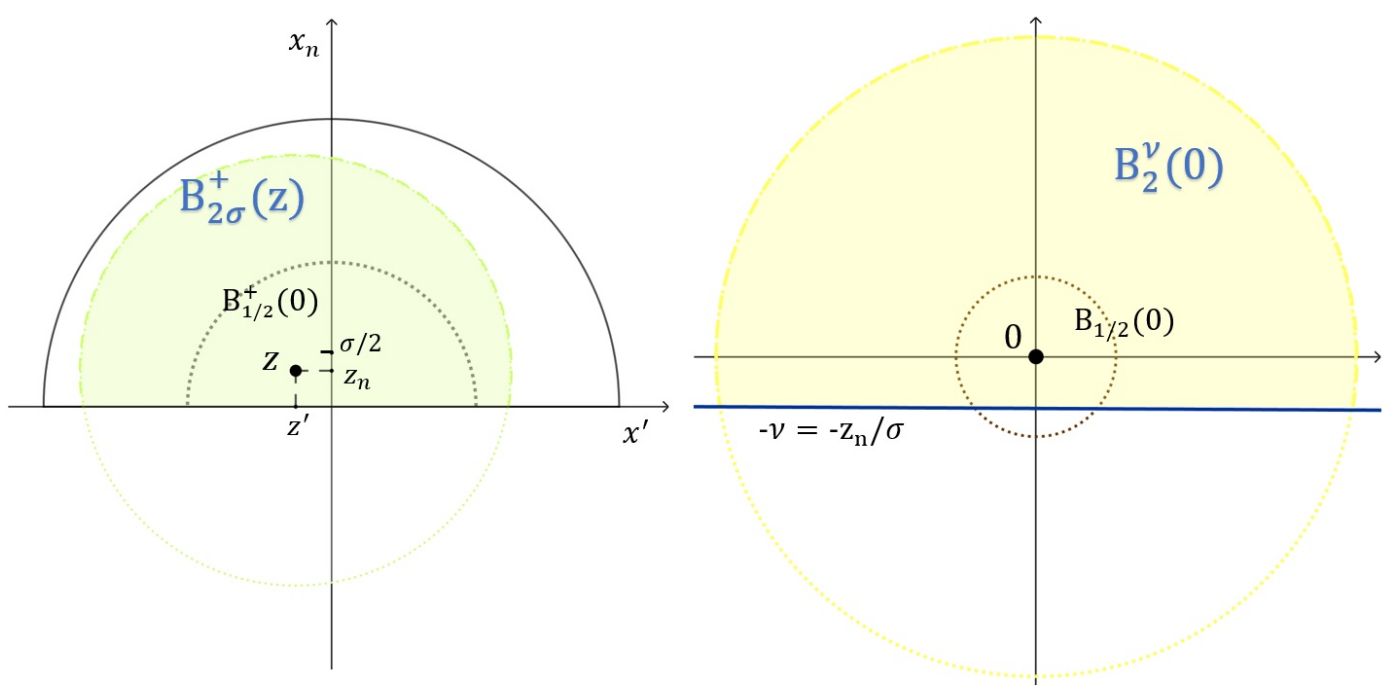

Figure 3.1: Illustration of the variable change, from $B_{2 \sigma}^{+}(z)$ to $B_{2}^{\nu}(0)$.

Then we define $N=N_{\sigma}(z):=\sigma W+\sup _{x \in B_{2}^{\nu}(0)}|u(\sigma x+z)-u(z)|$. The $C^{\beta}$ estimate, this time the global one, restricted to the set $B_{2 \sigma}^{+}(z)$, yields

$$
\sigma W \leq N \leq\left(\sigma+2^{\beta} K_{1} \sigma^{\beta}\right) W \leq\left(1+2^{\beta} K_{1}\right) \sigma^{\beta} W_{0}
$$

Next we set $\widetilde{u}(x):=\frac{1}{N}\{u(\sigma x+z)-u(z)\}$. As in claim 3.9, $\widetilde{u}$ is an $L^{p}$-viscosity solution of

$$
\left\{\begin{array}{rlll}
\widetilde{F}\left(x, \widetilde{u}, D \widetilde{u}, D^{2} \widetilde{u}\right) & =\widetilde{f}(x) & \text { in } B_{2}^{\nu} \\
\widetilde{u} & =\widetilde{\psi} & \text { on } \quad \mathbb{T}_{2}^{\nu}
\end{array}\right.
$$

for

$\widetilde{F}(x, r, p, X):=\frac{\sigma^{2}}{N} F\left(\sigma x+z, N r+u(z), \frac{N}{\sigma} p, \frac{N}{\sigma^{2}} X\right)-\frac{\sigma^{2}}{N} F(\sigma x+z, u(z), 0,0)$, $\widetilde{\psi}(x):=\frac{1}{N}\{\psi(\sigma x+z)-u(z)\}$ and $\widetilde{f}:=\widetilde{f}_{1}+\widetilde{f}_{2}$ where

$$
\tilde{f}_{1}(x):=\sigma^{2} f(\sigma x+z) / N ; \quad \tilde{f}_{2}(x):=-\sigma^{2} F(\sigma x+z, u(z), 0,0) / N
$$

$\widetilde{F}$ satisfying $(\widetilde{S C})^{\tilde{\mu}}$ for $\widetilde{b}(x)=\sigma b(\sigma x+z), \widetilde{\mu}=N \mu, \widetilde{d}(x)=\sigma^{2} d(\sigma x+z)$ and $\widetilde{\omega}(r)=\omega(N r) / N$.

With this definition and the choice of $\sigma$ in (3.12), we get $\|\widetilde{u}\|_{L^{\infty}\left(B_{2}^{\nu}\right)} \leq 1$, $\|\widetilde{f}\|_{L^{p}\left(B_{2}^{\nu}\right)} \leq \frac{\delta}{8}, \widetilde{\mu} \leq \frac{\delta}{8 K^{2}\left|B_{1}\right|^{1 / p}},\|\widetilde{b}\|_{L^{p}\left(B_{2}^{\nu}\right)} \leq \frac{\delta}{16 K}, \widetilde{\omega}(1)\|\widetilde{d}\|_{L^{p}\left(B_{2}^{\nu}\right)} \leq \frac{\delta}{32\left(K_{4}+K+1\right)}$ and $\left\|\bar{\beta}_{\widetilde{F}}(0, \cdot)\right\|_{L^{p}\left(B_{1}^{\nu}\right)} \leq \delta / 4$ by choosing $\theta=\delta / 8$, as in the local case. 
Furthermore, we have $\|\widetilde{\psi}\|_{L^{\infty}\left(\mathbb{T}_{2}^{\nu}\right)} \leq\|\widetilde{u}\|_{L^{\infty}\left(B_{2}^{\nu}\right)} \leq 1$ and then

$$
\begin{aligned}
\|D \widetilde{\psi}\|_{C^{\tau}\left(\mathbb{T}_{2}^{\nu}\right)} & \leq \frac{\sigma}{N}\|D \psi\|_{L^{\infty}\left(B_{2 \sigma}(z) \cap \mathbb{T}\right)}+\frac{\sigma}{N} \sup _{x \neq y \in \mathbb{T}_{2}^{\nu}} \frac{|D \psi(\sigma x+z)-D \psi(\sigma y+z)|}{|\sigma x-\sigma y|^{\tau}} \sigma^{\tau} \\
& \leq \frac{\|\psi\|_{C^{1, \tau}(\mathbb{T})}}{W} \leq 1
\end{aligned}
$$

since $N \geq \sigma W$. Therefore, we obtain

$$
\|\widetilde{\psi}\|_{C^{1, \tau}\left(\mathbb{T}_{2}^{\nu}\right)}=\|\widetilde{\psi}\|_{L^{\infty}\left(\mathbb{T}_{2}^{\nu}\right)}+\|D \widetilde{\psi}(x)\|_{C^{\tau}\left(\mathbb{T}_{2}^{\nu}\right)} \leq 2
$$

We can suppose, up to this rescaling, that $F, u, \mu, b, d, \omega$ satisfy the former hypotheses related to $\widetilde{F}, \widetilde{u}, \widetilde{\mu}, \widetilde{b}, \widetilde{d}, \widetilde{\omega}$. Thus, we move to the construction of $l_{k}(x):=a_{k}+b_{k} \cdot x$ such that

$$
\begin{aligned}
& (i)_{k} \quad\left\|u-l_{k}\right\|_{L^{\infty}\left(B_{r_{k}}^{\nu}\right)} \leq r_{k}^{1+\alpha} \\
& (i i)_{k}\left|a_{k}-a_{k-1}\right| \leq K_{4} r_{k-1}^{1+\alpha}, \quad\left|b_{k}-b_{k-1}\right| \leq K_{4} r_{k-1}^{\alpha} \\
& (i i i)_{k}\left|\left(u-l_{k}\right)\left(r_{k} x\right)-\left(u-l_{k}\right)\left(r_{k} y\right)\right| \leq C_{1,4} r_{k}^{1+\alpha}|x-y|^{\beta} \quad \text { for all } x, y \in B_{1}^{\nu_{k}}
\end{aligned}
$$
where $C_{1,4}=C_{1,4}\left(K_{1}, K_{4}\right)$ and $\nu_{k}:=\frac{\nu}{r_{k}}, r_{k}=\gamma^{k}$ for some $\gamma \in(0,1)$, for all $k \geq 0$; again $l_{-1} \equiv 0$.

We emphasize that these iterations will prove that the function $u$ (which plays the role of $\widetilde{u}$ ) is differentiable at 0 and provide

$$
|u(x)-u(0)-D u(0) \cdot x| \leq C|x|^{1+\alpha}, \quad|D u(0)| \leq C
$$

for every $x \in B_{1}^{\nu}$. In terms of our original function defined on $B_{1}^{+}$, it means that $u$ will be differentiable at $z$, for all $z$ with $z_{n}<\frac{\sigma}{2}$. On the other hand, the second case $z_{n} \geq \frac{\sigma}{2}$ is covered by the local part, section 3.2.1, since in this situation we are far away from the bottom boundary. Consequently, boundary superlinear regularity and estimates on $B_{1}^{+}$will follow by a covering argument.

For the proof of $(i)_{k}-(i i i)_{k}$, we use induction on $k$. For $k=0$ we set $a_{0}=b_{0}=0$. Recall that $\beta$ and $K_{1}$ are the constants from $C^{\beta}$ quadratic global estimate in the set $B_{1}^{\nu}$, then we have $\|u\|_{C^{\beta}\left(\overline{B_{1}^{\nu}}\right)} \leq \widetilde{K}_{1}(1+\delta+2+1) \leq 5 K_{1}$ and so $(i i i)_{0}$ for $\nu_{0}=\nu,(i)_{0}$ and $(i i)_{0}$ are valid.

Analogously to the the local case, we have $\left|b_{k}\right|,\left\|l_{k}\right\|_{L^{\infty}\left(B_{r_{k}}^{\nu}\right)} \leq K$. For the induction's step we suppose $(i)_{k}-(i i i)_{k}$ and construct $a_{k+1}, b_{k+1}$ such that $(i)_{k+1}-(i i i)_{k+1}$ are valid. Define

$$
v(x)=v_{k}(x):=\frac{\left(u-l_{k}\right)\left(r_{k} x\right)}{r_{k}^{1+\alpha}}=\frac{u\left(r_{k} x\right)-a_{k}-b_{k} \cdot x r_{k}}{r_{k}^{1+\alpha}}, \text { for all } x \in B_{2}^{\nu_{k}} .
$$


Since $r_{k} x \in B_{r_{k}}^{\nu} \Leftrightarrow x \in B_{1}^{\nu_{k}},(i)_{k}$ says that $|v| \leq 1$ in $B_{1}^{\nu_{k}}$. From this and $(i i i)_{k}$,

$$
\|v\|_{C^{\beta}\left(\overline{\left.B_{1}^{\nu}\right)}\right.}=\|v\|_{L^{\infty}\left(B_{1}^{\nu_{k}}\right)}+\sup _{\substack{x, y \in B_{1}^{\nu_{k}} \\ x \neq y}} \frac{|v(x)-v(y)|}{|x-y|^{\beta}} \leq 1+C_{1,4}=: K_{0} .
$$

Notice that, as in claim $3.12, v$ is an $L^{p}$-viscosity solution of

$$
\left\{\begin{aligned}
F_{k}\left(x, v, D v, D^{2} v\right) & =f_{k}(x) \text { in } B_{2}^{\nu_{k}} \\
v & =\psi_{k} \quad \text { on } \mathbb{T}_{2}^{\nu_{k}}
\end{aligned}\right.
$$

for $f_{k}:=f_{k}^{1}+f_{k}^{2} ; f_{k}^{1}(x):=r_{k}^{1-\alpha} f\left(r_{k} x\right), f_{k}^{2}(x):=-r_{k}^{1-\alpha} F\left(r_{k} x, l_{k}\left(r_{k} x\right), b_{k}, 0\right)$ and $F_{k}(x, s, p, X)$, defined as

$$
r_{k}^{1-\alpha} F\left(r_{k} x, r_{k}^{1+\alpha} s+l_{k}\left(r_{k} x\right), r_{k}^{\alpha} p+b_{k}, r_{k}^{\alpha-1} X\right)-r_{k}^{1-\alpha} F\left(r_{k} x, l_{k}\left(r_{k} x\right), b_{k}, 0\right),
$$

satisfying $(S C)_{F_{k}}^{\mu_{F_{k}}}$ with coefficients $b_{F_{k}}(x)=r_{k} b\left(r_{k} x\right)+2 r_{k} \mu K, \mu_{F_{k}}=r_{k}^{1+\alpha} \mu$, $d_{F_{k}}(x)=r_{k}^{2} d\left(r_{k} x\right)$ and $\omega_{F_{k}}(s)=r_{k}^{-1-\alpha} \omega\left(r_{k}^{1+\alpha} s\right)$.

These $F_{k}, v, \mu_{F_{k}}, b_{F_{k}}, d_{F_{k}}, \omega_{F_{k}}$ still satisfy the hypotheses of lemma 3.15, since $\left\|b_{F_{k}}\right\|_{L^{p}\left(B_{1}^{\nu_{k}}\right)} \leq \delta, \omega_{F_{k}}(1)\left\|d_{F_{k}}\right\|_{L^{p}\left(B_{1}^{\nu k}\right)} \leq \delta,\left\|f_{k}\right\|_{L^{p}\left(B_{1}^{\nu}\right)} \leq \delta$ and $\left\|\bar{\beta}_{F_{k}}(\cdot, 0)\right\|_{L^{p}\left(B_{1}^{\nu}\right)} \leq \delta$, see section 3.2.1.

Let $h=h_{k} \in C\left(\overline{B_{1}^{\nu_{k}}}\right)$ be the $C$-viscosity solution of

$$
\left\{\begin{array}{rlll}
F_{k}\left(0,0,0, D^{2} h\right) & =0 & & \text { in } \quad B_{1}^{\nu_{k}} \\
h & =v & \text { on } \partial B_{1}^{\nu_{k}}
\end{array}\right.
$$

given by proposition 2.23, since $B_{1}^{\nu_{k}}$ has the uniform exterior cone condition. From ABP we get $\|h\|_{L^{\infty}\left(B_{1}^{\nu_{k}}\right)} \leq\|h\|_{L^{\infty}\left(\partial B_{1}^{\nu_{k}}\right)} \leq 1$. Further, $h=v=\psi_{k} \in$ $C^{1, \tau}\left(B_{1} \cap\left\{x_{n}=-\nu_{k}\right\}\right)$ and we can find a uniform bound for the $C^{1, \tau}$ norm of $\psi_{k}$. Indeed, $\left\|\psi_{k}\right\|_{L^{\infty}\left(\mathbb{T}_{1}^{\nu_{k}}\right)} \leq\|v\|_{L^{\infty}\left(\overline{B_{1}^{\nu / k}}\right)} \leq 1$ and

$$
\left[D \psi_{k}\right]_{\tau, \mathbb{T}_{1}^{\nu_{k}}}=\sup _{\substack{x, y \in \mathbb{T}_{1}^{\nu_{k}} \\ x \neq y}} \frac{\left|D \psi_{k}(x)-D \psi_{k}(y)\right|}{|x-y|^{\tau}}=\sup _{\substack{\tilde{x}, \tilde{y} \in \mathbb{T}_{k}^{\nu} \\ \tilde{x}=r_{k} x, \tilde{y}=r_{k} y}} \frac{|D \psi(\tilde{x})-D \psi(\tilde{y})|}{|\tilde{x}-\tilde{y}|^{\tau}} r_{k}^{\tau-\alpha} \leq 1
$$

since $\|D \psi\|_{C^{\tau}\left(\mathbb{T}_{1}^{\nu}\right)} \leq 1$ and $\alpha \leq \tau$. Moreover, using the global Holder interpolation in smooth domains, lemma 6.35 of [41], for $\epsilon=\frac{1}{2}$, there exists a constant $C_{n}$, which does not depend ${ }^{1}$ on $k$, such that

$$
\left\|\psi_{k}\right\|_{C^{1}\left(\mathbb{T}_{1}^{\nu k}\right)} \leq C_{n}\left\|\psi_{k}\right\|_{C\left(\mathbb{T}_{1}^{\nu k}\right)}+\frac{1}{2}\left\|\psi_{k}\right\|_{C^{1, \tau}\left(\mathbb{T}_{1}^{\nu k}\right)}
$$

${ }^{1}$ The proof of lemma 6.35 in [41] is based on an interpolation inequality (6.89) for adimensional Holder norms (that does not depend on the domain); followed by a partition of unity that straightens the boundary (not necessary in our case $\mathbb{T}_{1}^{\nu_{k}} \subset \mathbb{R}^{n-1}$ ). 
hence

$$
\left\|\psi_{k}\right\|_{C^{1, \tau}\left(\mathbb{T}_{1}^{\nu_{k}}\right)}=\left\|\psi_{k}\right\|_{C^{1}\left(\mathbb{T}_{1}^{\nu_{k}}\right)}+\left[D \psi_{k}\right]_{\tau, \mathbb{T}_{1}^{\nu_{k}}} \leq C_{n}+\frac{1}{2}\left\|\psi_{k}\right\|_{C^{1, \tau}\left(\mathbb{T}_{1}^{\nu_{k}}\right)}+1
$$

i.e. $\left\|\psi_{k}\right\|_{C^{1, \tau}\left(\mathbb{T}_{1}^{\nu}\right)} \leq 2\left(C_{n}+1\right)$. Thus, the $C^{1, \bar{\alpha}}$ global estimate (proposition 2.24 ) yields

$$
\|h\|_{C^{1, \bar{\alpha}}\left(\overline{\left.B_{1 / 2}^{\nu}\right)}\right.} \leq K_{3}\left\{\|h\|_{L^{\infty}\left(B_{1}^{\nu_{k}}\right)}+\left\|\psi_{k}\right\|_{C^{1, \tau}\left(\mathbb{T}_{1}^{\nu_{k}}\right)}\right\} \leq K_{3}\left(3+2 C_{n}\right)=: K_{4} .
$$

Now, the approximation boundary lemma 3.8 applied to $F_{k}, v, h, \nu_{k}, \mu_{F_{k}}, b_{F_{k}}$, $d_{F_{k}}, \omega_{F_{k}}, \psi_{k}, \beta, K_{0}$ gives us that $\|v-h\|_{L^{\infty}\left(B_{1}^{\nu_{k}}\right)} \leq \varepsilon$.

Therefore, defining $\bar{l}(x)=\bar{l}_{k}(x):=h(0)+D h(0) \cdot x$ in $B_{1}^{\nu_{k}}$, it follows that

$$
\|v-\bar{l}\|_{L^{\infty}\left(B_{2 \gamma}^{\nu_{k}}\right)} \leq \gamma^{1+\alpha}
$$

In fact, by the choice of $\gamma$ we have, for all $x \in B_{2 \gamma}^{\nu_{k}}(0)$,

$$
|v(x)-\bar{l}(x)| \leq|v(x)-h(x)|+|h(x)-h(0)-D h(0) \cdot x| \leq 2 K_{4}(2 \gamma)^{1+\bar{\alpha}} \leq \gamma^{1+\alpha}
$$

Next, (3.20) and the definition of $v$ imply

$$
\left|u\left(r_{k} x\right)-l_{k}\left(r_{k} x\right)-r_{k}^{1+\alpha} h(0)-r_{k}^{1+\alpha} D h(0) \cdot x\right| \leq r_{k}^{1+\alpha} \gamma^{1+\alpha}=r_{k+1}^{1+\alpha} \text { for } x \in B_{2 \gamma}^{\nu_{k}}
$$

which is equivalent to

$$
\left|u(y)-l_{k+1}(y)\right| \leq r_{k}^{1+\alpha} \gamma^{1+\alpha}=r_{k+1}^{1+\alpha} \quad \text { for all } y=r_{k} x \in B_{2 \gamma r_{k}}^{\nu}=B_{2 r_{k+1}}^{\nu}
$$

where $l_{k+1}(y):=l_{k}(y)+r_{k}^{1+\alpha} h(0)+r_{k}^{\alpha} D h(0) \cdot y$. Then, we define $a_{k+1}:=a_{k}+$ $h(0) r_{k}^{1+\alpha}, b_{k+1}:=b_{k}+D h(0) r_{k}^{\alpha}$, obtaining $(i)_{k+1}$. Also, $\left|a_{k+1}-a_{k}\right| \leq K_{4} r_{k}^{1+\alpha}$, $\left|b_{k+1}-b_{k}\right| \leq K_{4} r_{k}^{\alpha}$, which is $(i i)_{k+1}$. As in the local case, to finish the proof of $(\text { iii })_{k+1}$, it is enough to show that

$$
\|v-\bar{l}\|_{C^{\beta}\left(\overline{B_{\gamma}^{\nu}}\right)} \leq C_{1,4} \gamma^{1+\alpha-\beta} .
$$

Let us see that this is obtained by applying the global superlinear $C^{\beta}$ estimate (proposition 2.14) to the function $w:=v-\bar{l}$.

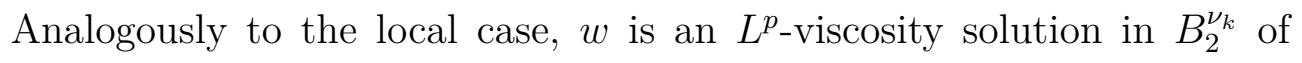
(3.17) (see notations and coefficients there), in addition to $w=\psi_{k}-\bar{l}$ on $\mathbb{T}_{2}^{\nu_{k}}$. The definition of $\delta$ gives us $\left\|g_{k}\right\|_{L^{p}\left(B_{1}^{\nu_{k}}\right)} \leq\left(5+2 K_{4}\right) \delta \leq \gamma^{\alpha}$. Further, using that $\psi_{k}=h$ on $\mathbb{T}_{2 \gamma}^{\nu_{k}}$, we obtain $\left\|\psi_{k}-\bar{l}\right\|_{L^{\infty}\left(\mathbb{T}_{2 \gamma}^{\nu_{k}}\right)} \leq \gamma^{1+\alpha}$.

Now, since $\psi_{k}-\bar{l} \in C^{1}\left(\mathbb{T}_{2 \gamma}^{\nu_{k}}\right)$, it is a Lipschitz function with constant less 
or equal than $\left\|D \psi_{k}-D \bar{l}\right\|_{C\left(\mathbb{T}_{2 \gamma}^{\nu k}\right)} \leq 2\left(C_{n}+1\right)+K_{4} \leq 2 K_{4}$ and thus

$$
\begin{aligned}
\mid\left(\psi_{k}-\bar{l}\right)(x) & -\left(\psi_{k}-\bar{l}\right)(y) \mid \\
& =\left|\left(\psi_{k}-\bar{l}\right)(x)-\left(\psi_{k}-\bar{l}\right)(y)\right|^{\tau}\left|\left(\psi_{k}-\bar{l}\right)(x)-\left(\psi_{k}-\bar{l}\right)(y)\right|^{1-\tau} \\
& \leq\left(2 K_{4}\right)^{\tau}\left(2 K_{4}\right)^{1-\tau}|x-y|^{\tau} \gamma^{(1+\bar{\alpha})(1-\tau)} \\
& =2 K_{4}|x-y|^{\tau} \gamma^{1-\tau+\bar{\alpha}(1-\tau)}
\end{aligned}
$$

Then, the choice of $\alpha$ implies that $\left[\psi_{k}-\bar{l}\right]_{\tau, \mathbb{T}_{2 \gamma}^{\nu k}} \leq 4 K_{4} \gamma^{1-\tau+\alpha}$. Hence, from this, (3.20) and $C^{\beta}$ global estimate, properly scaled for the radius $\gamma$, we obtain

$$
\begin{aligned}
{[w]_{\beta, \overline{B_{\gamma}^{\nu}}} \leq } & \gamma^{-\beta} \widetilde{K}_{1}\left\{\|w\|_{L^{\infty}\left(B_{2 \gamma}^{\nu_{k}}\right)}+\gamma^{2-\frac{n}{p}}\left\|g_{k}\right\|_{L^{p}\left(B_{2 \gamma}^{\nu_{k}}\right)}\right. \\
& \left.+\left\|\psi_{k}-\bar{l}\right\|_{L^{\infty}\left(\mathbb{T}_{2 \gamma}^{\nu_{k}}\right)}+\gamma^{\tau}\left[\psi_{k}-\bar{l}\right]_{\tau, \mathbb{T}_{2 \gamma}^{\nu_{k}}}\right\} \\
\leq & \gamma^{-\beta} K_{1}\left\{2 \gamma^{1+\alpha}+\gamma^{2-\frac{n}{p}} \gamma^{\alpha}+4 K_{4} \gamma^{1+\alpha}\right\} \leq K_{1}\left(3+4 K_{4}\right) \gamma^{1+\alpha-\beta}
\end{aligned}
$$

and finally, for $C_{1,4}:=1+\left(3+4 K_{4}\right) K_{1}=C_{1,4}\left(K_{1}, K_{4}\right)$, we conclude

$$
\begin{aligned}
\|w\|_{C^{\beta}\left(\overline{B_{\gamma}^{\nu}}\right)} & =\|w\|_{L^{\infty}\left(B_{\gamma}^{\nu_{k}}\right)}+[w]_{\beta, \overline{B_{\gamma}^{\nu}}} \\
& \leq \gamma^{1+\alpha}+\left(3+4 K_{4}\right) K_{1} \gamma^{1+\alpha-\beta} \leq C_{1,4} \gamma^{1+\alpha-\beta} .
\end{aligned}
$$

\subsection{3}

\section{The Global Blend}

Our goal in this section is to prove the global statement of theorem 3.1, by combining the local regularity estimates derived in section 3.2.1 with theorem 3.14 in section 3.2.2. Even though the argument is easily assembled from the previous constructions, we compile the details for completeness since, on the one hand, some technical difficulties arise due to the presence of quadratic growth in the gradient; on the other hand, it permits to clarify some points in the linear growth context in [38].

Let us deal with the model equation (ME) for $L=F$. More precisely, assume that $u \in C(\bar{\Omega})$ is an $L^{p}$-viscosity solution of

$$
\left\{\begin{aligned}
F\left(x, u, D u, D^{2} u\right)+c(x) u+\langle M(x) D u, D u\rangle & =f(x) & \text { in } \Omega \\
u & =\psi & \text { on } \partial \Omega
\end{aligned}\right.
$$

for $\psi \in C^{1, \tau}(\partial \Omega)$ and $F$ satisfying (SC). Meanwhile we quickly discuss the changes when we have an arbitrary superlinear growth (since the latter is easier to handle when we are interested in diffeomorphic invariance property). 
From section 3.2.1 we obtain local regularity estimates for any $\Omega^{\prime} \subset \subset \Omega$, with a fixed distance from the boundary. So, as in [38], we only need to make a careful analysis on the neighborhoods of boundary points. In fact, in order to use theorem 3.14, near a boundary point we will make a diffeomorphic change of independent variable, which takes a neighborhood of $\partial \Omega$ into $B_{1}^{+}$. This change only depends on the coefficients of the equation and on the $C^{1,1}$ character of the boundary.

Let $x_{0} \in \partial \Omega$. Since $\partial \Omega \in C^{1,1}$, there exists an open set $\mathcal{U}_{x_{0}}$ containing $x_{0}$ and a $C^{1,1}$ diffeomorphism

$$
\begin{aligned}
\Phi: \quad \mathcal{U}_{x_{0}} \subset \mathbb{R}^{n} \longrightarrow B_{1}(0) \subset \mathbb{R}^{n} \\
x=\left(x_{1}, \ldots, x_{n}\right) \longmapsto\left(\Phi_{1}(x), \ldots, \Phi_{n}(x)\right)
\end{aligned}
$$

such that $\Phi\left(x_{0}\right)=0$ and $\Phi\left(\mathcal{U}_{x_{0}} \cap \Omega\right)=B_{1}^{+}, \Phi\left(\overline{\mathcal{U}}_{x_{0}} \cap \partial \Omega\right)=\mathbb{T}_{1}=B_{1} \cap\left\{x_{n}=0\right\}$.

Note that $u \in C(\bar{\Omega})$ is, in particular, an $L^{p}$-viscosity solution of the first equation in (3.21) in the smaller domain $\mathcal{U}_{x_{0}} \cap \Omega$, with $u=\psi$ on $\partial \Omega \cap \overline{\mathcal{U}}_{x_{0}}$. Also, the function $\widetilde{u}:=u \circ \Phi^{-1}$ is continuous in $\overline{B_{1}^{+}}$.

We claim that $\widetilde{u}$ is an $L^{p}$-viscosity solution of

$$
\left\{\begin{array}{rll}
\widetilde{F}\left(x, \widetilde{u}, D \widetilde{u}, D^{2} \widetilde{u}\right)+\widetilde{c}(x) \widetilde{u}+\langle\widetilde{M}(x) D \widetilde{u}, D \widetilde{u}\rangle & =\widetilde{f}(x) & \text { in } B_{1}^{+} \\
\widetilde{u} & =\widetilde{\psi} & \text { on } \quad \mathbb{T}_{1}
\end{array}\right.
$$

where $\widetilde{c}:=c \circ \Phi^{-1}, \widetilde{f}:=f \circ \Phi^{-1}, \widetilde{M}:=\left(D \Phi^{T} \circ \Phi^{-1}\right) M \circ \Phi^{-1}\left(D \Phi \circ \Phi^{-1}\right)$, $\widetilde{\psi}:=\psi \circ \Phi^{-1}$ and $\widetilde{F}(x, r, p, X)$, defined as

$$
\left.F\left(\Phi^{-1}(x), r,\left(D \Phi \circ \Phi^{-1}\right) p,\left(D \Phi^{T} \circ \Phi^{-1}\right) X\left(D \Phi \circ \Phi^{-1}\right)+\left(\left(\partial_{i j} \Phi \circ \Phi^{-1}\right) p\right)_{i, j}\right)\right),
$$

satisfies $(\widetilde{S C})$, with ellipticity coefficients $\tilde{\lambda}=\lambda c_{\Phi}^{1}, \tilde{\Lambda}=\Lambda c_{\Phi}^{2}$, for some positive constants $c_{\Phi}^{1}, c_{\Phi}^{2}, \widetilde{b}:=\left\|D \Phi \circ \Phi^{-1}\right\|_{\infty} b \circ \Phi^{-1}+n^{2} \Lambda \max _{1 \leq i, j \leq n}\left|\partial_{i j} \Phi \circ \Phi^{-1}\right|$, $\widetilde{d}:=d \circ \Phi^{-1}$ and $\widetilde{\omega}:=\omega$. Moreover,

$$
\beta_{\widetilde{F}}\left(x, x_{0}\right) \leq C_{\Phi}^{1} \beta_{F}\left(\Phi^{-1}(x), \Phi^{-1}\left(x_{0}\right)\right)+C_{\Phi}^{2}\left|x-x_{0}\right|
$$

and

$$
\bar{\beta}_{\widetilde{F}}\left(x, x_{0}\right) \leq C_{\Phi}^{3} \bar{\beta}_{F}\left(\Phi^{-1}(x), \Phi^{-1}\left(x_{0}\right)\right)+C_{\Phi}^{2}\left|x-x_{0}\right|,
$$

for some positive constants $C_{\Phi}^{1}, C_{\Phi}^{2}$ and $C_{\Phi}^{3}$. Furthermore, $\widetilde{F}$ is convex or concave provided $F$ is (notice that this last statement is important to deal with the $W^{2, p}$ results).

Let us prove the claim. Of course $\widetilde{c}, \tilde{d} \tilde{f} \in L^{p}\left(B_{1}^{+}\right), \widetilde{\psi} \in C^{1, \tau}(\mathbb{T})$ and 
$\widetilde{u}=\widetilde{\psi}$ on $\mathbb{T}$. Observe that $\|M\|_{L^{\infty}\left(B_{1}^{+}\right)} \leq C(\Phi)\|M\|_{L^{\infty}(\Omega)}$.

Let $\widetilde{\varphi} \in W_{\text {loc }}^{2, p}\left(B_{1}^{+}\right)$a test function and set $\varphi:=\widetilde{\varphi} \circ \Phi \in W_{\text {loc }}^{2, p}\left(\mathcal{U}_{x_{0}} \cap \Omega\right)$. Since $\Phi \in C^{1,1}$, it follows that $D \Phi$ is Lipschitz and so differentiable a.e., then the second order partial derivatives of $\Phi$ exist at almost every point. We know (for instance, theorem 4 in section 5.8 of [29]) that $D \Phi \in W^{1, \infty}(\Omega)$, so $\Phi \in W^{2, q}(\Omega)$ for all $q \leq+\infty$. Thus $\widetilde{b} \in L^{p}(\Omega)$ and we can calculate

$$
\begin{aligned}
\partial_{i} \varphi(x) & =\sum_{k=1}^{n} \partial_{k} \widetilde{\varphi}(\Phi(x)) \frac{\partial \Phi_{k}}{\partial x_{i}}(x) \\
\partial_{i j} \varphi(x) & =\sum_{k=1}^{n} \sum_{l=1}^{n} \partial_{k l} \widetilde{\varphi}(\Phi(x)) \frac{\partial \Phi_{l}}{\partial x_{j}} \frac{\partial \Phi_{k}}{\partial x_{i}}+\sum_{k=1}^{n} \partial_{k} \widetilde{\varphi}(\Phi(x)) \frac{\partial^{2} \Phi_{k}}{\partial x_{i} x_{j}}
\end{aligned}
$$

and then

$$
\begin{aligned}
D \varphi & =D \Phi(D \widetilde{\varphi} \circ \Phi) \in \mathbb{M}_{n \times 1}(\mathbb{R}) \\
D^{2} \varphi & =D \Phi^{T}\left(D^{2} \widetilde{\varphi} \circ \Phi\right) D \Phi+\left(\partial_{i j} \Phi(D \widetilde{\varphi} \circ \Phi)\right)_{1 \leq i, j \leq n} \in \mathbb{M}_{n \times n}(\mathbb{R})
\end{aligned}
$$

under the convention that $\partial_{i j} \Phi$ is the line matrix in $\mathbb{M}_{1 \times n}(\mathbb{R})$ given by the second order derivatives in direction $i, j$ of the coordinate functions $\Phi_{k}$, i.e. $\partial_{i j} \Phi=\left(\frac{\partial^{2} \Phi_{1}}{\partial x_{i} x_{j}} \cdots \frac{\partial^{2} \Phi_{n}}{\partial x_{i} x_{j}}\right)$. In order to give meaning to the quadratic form $\langle M(x) p, p\rangle$, for a matrix $M(x) \in \mathbb{M}_{n \times n}(\mathbb{R})$, we make the convention that vector derivatives of functions from $\mathbb{R}^{n}$ into $\mathbb{R}$ are column matrices in $\mathbb{M}_{n \times 1}(\mathbb{R})$.

Observe that the definition on the coefficients of the equation satisfied by $\widetilde{u}$ in (3.22) is the only one that makes sense when we evaluate our original equation (3.21) with $u$, on the derivatives of the test function $\varphi$, and apply $\Phi^{-1}$ to obtain the desired equation in $B_{1}^{+}$, i.e.

$$
\begin{gathered}
F\left(x, u, D \varphi, D^{2} \varphi\right) \circ \Phi^{-1}=F\left(\Phi^{-1}(x), u \circ \Phi^{-1},\left(D \Phi \circ \Phi^{-1}\right) D \widetilde{\varphi},\right. \\
\left.\left(D \Phi^{T} \circ \Phi^{-1}\right) D^{2} \widetilde{\varphi}\left(D \Phi \circ \Phi^{-1}\right)+\left(\left(\partial_{i j} \Phi \circ \Phi^{-1}\right) D \widetilde{\varphi}\right)_{i, j}\right) \\
=\widetilde{F}\left(x, \widetilde{u}, D \widetilde{\varphi}, D^{2} \widetilde{\varphi}\right)
\end{gathered}
$$

and

$$
\begin{aligned}
\langle M(x) D \varphi, D \varphi\rangle \circ \Phi^{-1} & =\left\langle\left(M \circ \Phi^{-1}\right)(x)\left(D \Phi \circ \Phi^{-1}\right) D \widetilde{\varphi},\left(D \Phi \circ \Phi^{-1}\right) D \widetilde{\varphi}\right\rangle \\
& =\left(\left(D \Phi \circ \Phi^{-1}\right) D \widetilde{\varphi}\right)^{T}\left(M \circ \Phi^{-1}\right)(x)\left(\left(D \Phi \circ \Phi^{-1}\right) D \widetilde{\varphi}\right) \\
& =(D \widetilde{\varphi})^{T}\left(\left(D \Phi \circ \Phi^{-1}\right)^{T}\left(M \circ \Phi^{-1}\right)(x)\left(D \Phi \circ \Phi^{-1}\right)\right)(D \widetilde{\varphi}) \\
& =\langle\widetilde{M}(x) D \widetilde{\varphi}, D \widetilde{\varphi}\rangle .
\end{aligned}
$$


Notice that

$$
\begin{aligned}
& \sup _{\lambda I \leq A=\left(a_{i j}\right)_{i j} \leq \Lambda I} \operatorname{tr}\left(A\left(\partial_{i j} \Phi \circ \Phi^{-1}\right)(p-q)\right) \\
& =\sup _{\lambda I \leq A \leq \Lambda I} \sum_{i, j=1}^{n}\left|a_{i j}\left(\partial_{i j} \Phi \circ \Phi^{-1}\right)(p-q)\right| \leq n^{2} \Lambda \max _{1 \leq i, j \leq n}\left|\partial_{i j} \Phi \circ \Phi^{-1}\right||p-q|
\end{aligned}
$$

and

$$
\begin{aligned}
& \sup _{\lambda I \leq A \leq \Lambda I} \operatorname{tr}\left(A\left(D \Phi^{T} \circ \Phi^{-1}\right) X\left(D \Phi \circ \Phi^{-1}\right)\right) \\
& \quad=\sup _{\lambda I \leq A \leq \Lambda I} \operatorname{tr}\left(\left(D \Phi \circ \Phi^{-1}\right) A\left(D \Phi^{T} \circ \Phi^{-1}\right) X\right)=\sup _{\tilde{\lambda} I \leq \tilde{A} \leq \tilde{\Lambda} I} \operatorname{tr}(\tilde{A} X),
\end{aligned}
$$

since $\langle\tilde{A} \xi, \xi\rangle=\xi^{T}\left(D \Phi \circ \Phi^{-1}\right) A\left(D \Phi^{T} \circ \Phi^{-1}\right) \xi=\left(D \Phi^{T} \circ \Phi^{-1} \xi\right)^{T} A\left(D \Phi^{T} \circ \Phi^{-1} \xi\right) \in$ $[\lambda, \Lambda]|\eta|^{2} \subset[\tilde{\lambda}, \tilde{\Lambda}]|\xi|^{2}$, for $\eta=D \Phi^{T} \circ \Phi^{-1} \xi \Leftrightarrow \xi=D \Phi \circ \Phi^{-1} \eta$ which is such that $\left\|D \Phi \circ \Phi^{-1}\right\|_{\infty}^{-1}|\xi| \leq|\eta| \leq\left\|D \Phi^{T} \circ \Phi^{-1}\right\|_{\infty}|\xi|$. Then, define $c_{\Phi}^{1}$ and $c_{\Phi}^{2}$, respectively, as the minimum and maximum of the numbers $\left\|D \Phi \circ \Phi^{-1}\right\|_{\infty}^{-2}$ and $\left\|D \Phi^{T} \circ \Phi^{-1}\right\|_{\infty}^{2}$, both being positive since the determinant of the Jacobian matrix of $\Phi$ is equal to 1 .

Therefore, $\widetilde{F}$ satisfies $(\widetilde{S C})$, since

$$
\begin{aligned}
& \widetilde{F}(x, r, p, X)-\widetilde{F}(x, s, q, Y) \\
& \left.=F\left(\Phi^{-1}(x), r,\left(D \Phi \circ \Phi^{-1}\right) p,\left(D \Phi^{T} \circ \Phi^{-1}\right) X\left(D \Phi \circ \Phi^{-1}\right)+\left(\left(\partial_{i j} \Phi \circ \Phi^{-1}\right) p\right)_{i, j}\right)\right) \\
& \left.-F\left(\Phi^{-1}(x), s,\left(D \Phi \circ \Phi^{-1}\right) q,\left(D \Phi^{T} \circ \Phi^{-1}\right) Y\left(D \Phi \circ \Phi^{-1}\right)+\left(\left(\partial_{i j} \Phi \circ \Phi^{-1}\right) q\right)_{i, j}\right)\right) \\
& \left.\leq \mathcal{M}_{\lambda, \Lambda}^{+}\left(\left(D \Phi^{T} \circ \Phi^{-1}\right)(X-Y)\left(D \Phi \circ \Phi^{-1}\right)+\left(\left(\partial_{i j} \Phi \circ \Phi^{-1}\right)(p-q)\right)_{i, j}\right)\right) \\
& +b\left(\Phi^{-1}(x)\right)\left|\left(D \Phi \circ \Phi^{-1}\right)(p-q)\right|+d\left(\Phi^{-1}(x)\right) \omega\left((s-r)^{+}\right) \\
& \left.\leq \mathcal{M}_{\lambda c_{\Phi}^{1}, \Lambda c_{\Phi}^{2}}^{+}(X-Y)+\mathcal{M}_{\lambda, \Lambda}^{+}\left(\left(\partial_{i j} \Phi \circ \Phi^{-1}\right)(p-q)\right)_{i, j}\right) \\
& +\left\|D \Phi \circ \Phi^{-1}\right\|_{\infty} b\left(\Phi^{-1}(x)\right)|p-q|+d\left(\Phi^{-1}(x)\right) \omega\left((s-r)^{+}\right) \\
& \leq \mathcal{M}_{\tilde{\lambda}, \tilde{\Lambda}}^{+}(X-Y)+\widetilde{b}(x)|p-q|+\widetilde{d}(x) \widetilde{\omega}\left((s-r)^{+}\right)
\end{aligned}
$$

Of course the bounds on the left hand side of $(\widetilde{S C})$ are completely analogous. On the other hand, if we compute $F$ with $(S C)^{\mu}$, we directly obtain $\widetilde{F}$ satisfying $(S C)^{\tilde{\mu}}$ for $\tilde{\mu}:=\left\|D \Phi \circ \Phi^{-1}\right\|_{\infty}^{2} \mu$.

Now we prove that the oscillations of $\beta$ and $\bar{\beta}$ remain controlled after the change of variable. In this case we need extra care to deal with $\Phi$ at different points $x$ and $x_{0}$. This was first observed by Prof. Diego Moreira, who suggested the following argument. 
Set $Q_{x}:=D \Phi\left(\Phi^{-1}(x)\right)$, then $\widetilde{F}(x, 0,0, X)=F\left(\Phi^{-1}(x), 0,0, Q_{x}^{T} X Q_{x}\right)$, so

$$
\begin{aligned}
\mid \widetilde{F}(x, 0,0, X) & -\widetilde{F}\left(x_{0}, 0,0, X\right) \mid \\
& =\left|F\left(\Phi^{-1}(x), 0,0, Q_{x}^{T} X Q_{x}\right)-F\left(\Phi^{-1}\left(x_{0}\right), 0,0, Q_{x_{0}}^{T} X Q_{x_{0}}\right)\right| \\
& \leq\left|F\left(\Phi^{-1}(x), 0,0, Q_{x}^{T} X Q_{x}\right)-F\left(\Phi^{-1}(x), 0,0, Q_{x_{0}}^{T} X Q_{x_{0}}\right)\right| \\
& +\left|F\left(\Phi^{-1}(x), 0,0, Q_{x_{0}}^{T} X Q_{x_{0}}\right)-F\left(\Phi^{-1}\left(x_{0}\right), 0,0, Q_{x_{0}}^{T} X Q_{x_{0}}\right)\right|
\end{aligned}
$$

The second addend above is bounded by

$$
\beta_{F}\left(\Phi^{-1}(x), \Phi^{-1}\left(x_{0}\right)\right)\left\|Q_{x_{0}}^{T} X Q_{x_{0}}\right\| \leq C_{\Phi}^{1} \beta_{F}\left(\Phi^{-1}(x), \Phi^{-1}\left(x_{0}\right)\right)\|X\|,
$$

where $C_{\Phi}^{1}:=\left\|D \Phi \circ \Phi^{-1}\right\|_{\infty}\left\|D \Phi^{T} \circ \Phi^{-1}\right\|_{\infty}$. For the first one, notice that $\left|F(x, 0,0, X)-F\left(x, 0,0, X_{0}\right)\right| \leq \max \left\{\mathcal{M}^{+}\left(X-X_{0}\right), \mathcal{M}^{+}\left(X_{0}-X\right)\right\}$, and

$$
\begin{aligned}
\mathcal{M}^{+}\left(Q_{x}^{T} X Q_{x}-Q_{x_{0}}^{T} X Q_{x_{0}}\right) & \leq \mathcal{M}^{+}\left(\left(Q_{x}^{T}-Q_{x_{0}}^{T}\right) X Q_{x}\right)+\mathcal{M}^{+}\left(Q_{x_{0}}^{T} X\left(Q_{x}-Q_{x_{0}}\right)\right) \\
& \leq C_{\Phi}^{2}\left|x-x_{0}\right|\|X\|,
\end{aligned}
$$

where $C_{\Phi}^{2}:=n \Lambda \max \left\{\left\|D \Phi \circ \Phi^{-1}\right\|_{\infty},\left\|D \Phi^{T} \circ \Phi^{-1}\right\|_{\infty}\right\}\left\{\operatorname{Lip}\left(D \Phi \circ \Phi^{-1}\right)+\right.$ $\left.\operatorname{Lip}\left(D \Phi^{T} \circ \Phi^{-1}\right)\right\}$, hence (3.23) is proved. On the other hand, using that (3.26) is less or equal than $C_{\Phi}^{2}\left|x-x_{0}\right|(\|X\|+1)$, and replacing (3.25) by

$$
\bar{\beta}_{F}\left(\Phi^{-1}(x), \Phi^{-1}\left(x_{0}\right)\right)\left(\left\|Q_{x_{0}}^{T} X Q_{x_{0}}\right\|+1\right) \leq C_{\Phi}^{3} \bar{\beta}_{F}\left(\Phi^{-1}(x), \Phi^{-1}\left(x_{0}\right)\right)(\|X\|+1),
$$

where $C_{\Phi}^{3}:=\max \left\{C_{\Phi}^{1}, 1\right\}$, we obtain $(3.24)$.

Finally, consider $\widetilde{F}_{x, r, p}=F_{\Phi^{-1}(x), r,\left(D \Phi \circ \Phi^{-1}\right) p}$ for fixed $(x, r, p)$. Then

$$
\begin{aligned}
& \widetilde{F}(t X+(1-t) Y) \\
& =F\left(t\left(D \Phi^{T} \circ \Phi^{-1}\right) X\left(D \Phi \circ \Phi^{-1}\right)+(1-t)\left(D \Phi^{T} \circ \Phi^{-1}\right) Y\left(D \Phi \circ \Phi^{-1}\right)\right) \\
& \lesseqgtr t F\left(\left(D \Phi^{T} \circ \Phi^{-1}\right) X\left(D \Phi \circ \Phi^{-1}\right)\right)+(1-t) F\left(\left(D \Phi^{T} \circ \Phi^{-1}\right) Y\left(D \Phi \circ \Phi^{-1}\right)\right) \\
& =t \widetilde{F}(X)+(1-t) \widetilde{F}(Y)
\end{aligned}
$$

for all $X, Y \in \mathbb{S}^{n}$ and $t \in[0,1]$, provided $F$ is convex or concave in the $X$ entry, and the claim is proved.

To finish we cover the boundary with a finitely many neighborhoods of this type to obtain regularity and an estimate near the boundary. In turn, the complete proof of regularity and estimates in the global case is derived by a covering argument over the domain $\Omega$, using local and boundary results. 


\section{3}

\section{$W^{2, p}$ Results}

In this section we prove the $W^{2, p}$ type results stated in section 3.1.

Proof of Theorem 3.4. We prove only the global case, since in the local one we just ignore the term with $\psi$, by considering it equal to zero in what follows. Notice that $\psi \in W^{2, p}(\Omega) \subset C^{1, \tau}(\bar{\Omega})$ for some $\tau \in(0,1)$ with continuous inclusion, then $\|u\|_{L^{\infty}(\Omega)}+\|f\|_{L^{p}(\Omega)}+\|\psi\|_{C^{1, \tau}(\partial \Omega)} \leq C_{2}$.

Thus, by $C^{1, \alpha}$ regularity theorem, we have that $\bar{f}(x):=f(x)-g(x, D u) \in$ $L^{p}(\Omega)$ and also

$$
\|u\|_{C^{1, \alpha}(\bar{\Omega})} \leq C_{3}\left\{\|u\|_{L^{\infty}(\Omega)}+\|\bar{f}\|_{L^{p}(\Omega)}+\|\psi\|_{C^{1, \tau}(\partial \Omega)}\right\}
$$

Claim $3.16 u$ is an $L^{p}$-viscosity solution of $F\left(x, u, D u, D^{2} u\right)=\bar{f}(x)$ in $\Omega$.

Proof of Claim 3.16. Let us prove the subsolution case; for the supersolution it is analogous. Assuming the contrary, there exists some $\phi \in W_{\text {loc }}^{2, p}(\Omega)$, $x_{0} \in \Omega$ and $\varepsilon>0$ such that $u-\phi$ has a local maximum at $x_{0}$ and $F\left(x, u, D \phi, D^{2} \phi\right)-\bar{f}(x) \leq-\varepsilon$ a.e. in $B_{r}\left(x_{0}\right)$.

In turn, by the definition of $u$ being an $L^{p}$-viscosity subsolution of (3.7),

$$
F\left(x, u, D \phi, D^{2} \phi\right)+g(x, D \phi) \geq f(x)-\varepsilon / 2 \text { a.e. in } B_{r}\left(x_{0}\right)
$$

up to diminishing $r>0$. By subtracting the last two inequalities, we obtain

$$
-\{\gamma+\mu(|D u|+|D \phi|)\}|D u-D \phi| \leq g(x, D u)-g(x, D \varphi) \leq-\varepsilon / 2
$$

a.e. in $B_{r}\left(x_{0}\right)$. Since $u-\phi \in C^{1}\left(B_{r}\left(x_{0}\right)\right)$ has a local maximum at $x_{0}$, we have $D(u-\phi)\left(x_{0}\right)=0$ and, moreover, $|D(u-\phi)(x)|<\varepsilon\left\{\gamma+\mu\left(\|D u\|_{L^{\infty}\left(B_{r}\left(x_{0}\right)\right)}+\right.\right.$ $\left.\left.\|D \phi\|_{L^{\infty}\left(B_{r}\left(x_{0}\right)\right.}\right)+1\right\}^{-1} / 4$ for all $x \in B_{r}\left(x_{0}\right)$, possibly for a smaller $r$, which contradicts (3.27).

Thus by Winter's result, theorem 4.3 in [38] (or Swięch [48] in the local case), we have that $u \in W^{2, p}(\Omega)$ (respectively $u \in W_{\text {loc }}^{2, p}(\Omega)$ ) and

$$
\begin{aligned}
\|u\|_{W^{2, p}(\Omega)} \leq & C\left\{\|u\|_{L^{\infty}(\Omega)}+\|\bar{f}\|_{L^{p}(\Omega)}+\|\psi\|_{W^{2, p}(\Omega)}\right\} \\
\leq & C\left\{\|u\|_{L^{\infty}(\Omega)}+\|f\|_{L^{p}(\Omega)}+\mu\|u\|_{C^{1}(\bar{\Omega})}^{2}+\gamma\|u\|_{C^{1}(\bar{\Omega})}+\|\psi\|_{W^{2, p}(\Omega)}\right\} \\
\leq & C\left\{\|u\|_{L^{\infty}(\Omega)}+\|f\|_{L^{p}(\Omega)}+\|\psi\|_{W^{2, p}(\Omega)}\right. \\
& \left.\quad+\left(\mu C_{2}+\gamma\right) C_{3}\left\{\|u\|_{L^{\infty}(\Omega)}+\|f\|_{L^{p}(\Omega)}+\|\psi\|_{C^{1, \tau}(\partial \Omega)}\right\}\right\}
\end{aligned}
$$

which implies the estimate.

In theorem 3.4, the final constants only depend on the $L^{p}$-norm of the coefficients, despite the boundedness hypothesis on $b, d$. The latter hypothesis 
is needed to conclude that solutions are twice differentiable a.e. Observe that, in [38] (see theorem 4.3 there), $W^{2, p}$ results consist of two parts: (i) introducing a new equation $F\left(x, 0,0, D^{2} u\right)=\tilde{f}(x)$ (via corollary 1.6 in [48]), in which $u$ remains a solution in the $L^{p}$-viscosity sense; (ii) obtaining $W^{2, p}$ estimates for solutions of $F\left(x, 0,0, D^{2} u\right)=\widetilde{f}(x)$, which are independent of the zero and first order coefficients.

Proof of Proposition 3.5. It is enough to treat the upper extremal case. Let $b_{k}, d_{k} \in L_{+}^{\infty}(\Omega)$ be such that $b_{k} \rightarrow b$ and $d_{k} \rightarrow d$ in $L^{p}(\Omega)$. Let $u_{k} \in W^{2, p}(\Omega)$ be the unique $L^{p}$-viscosity solution of

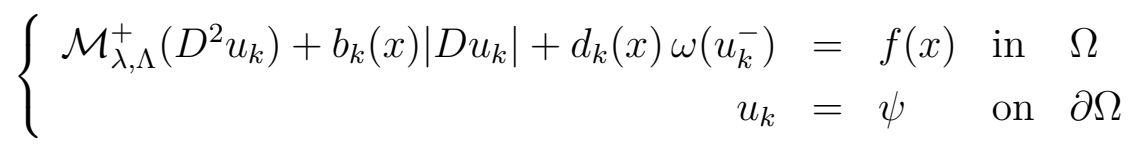

given by theorem 4.6 of [38]. From the estimates in theorem 3.4, we have

$$
\left\|u_{k}\right\|_{W^{2, p}(\Omega)} \leq C_{k}\left\{\left\|u_{k}\right\|_{L^{\infty}(\Omega)}+\|f\|_{L^{p}(\Omega)}+\|\psi\|_{W^{2, p}(\Omega)}\right\}
$$

where $C_{k}$ remains bounded, since $b_{k}$ and $d_{k}$ are bounded in $L^{p}(\Omega)$.

Now, by $\mathrm{ABP}$ we have that that $\left\|u_{k}\right\|_{L^{\infty}(\Omega)} \leq\|\psi\|_{L^{\infty}(\partial \Omega)}+C\|f\|_{L^{p}(\Omega)}$. From this and (3.28) we get $\left\|u_{k}\right\|_{W^{2, p}(\Omega)} \leq C$ and hence there exists $u \in C^{1}(\bar{\Omega})$ such that $u_{k} \rightarrow u$ in $C^{1}(\bar{\Omega})$.

Next, proposition 2.12 implies that $u$ is an $L^{p}$-viscosity solution of

$$
\left\{\begin{array}{rlll}
\mathcal{M}_{\lambda, \Lambda}^{+}\left(D^{2} u\right)+b(x)|D u|+d(x) \omega\left(u^{-}\right) & =f(x) & \text { in } & \Omega \\
u & =\psi & \text { on } & \partial \Omega .
\end{array}\right.
$$

Notice that $W^{2, p}(\Omega)$ is reflexive [30, p. 203, 264], so there exists $\tilde{u} \in W^{2, p}(\Omega)$ such that $u_{k}$ converges weakly to $\tilde{u}$. By uniqueness of the limit, $\tilde{u}=u$ a.e. in $\Omega$, and $u$ is a strong solution of (3.29).

Finally, if there exists another $L^{p}$-viscosity solution of (3.29), say $v \in$ $C(\bar{\Omega})$, then the function $w:=u-v$ satisfies $w=0$ on $\partial \Omega$ and it is an $L^{p}$-viscosity solution of $\mathcal{L}^{+}[w] \geq 0$ in $\Omega \cap\{w>0\}$. Indeed, since $u$ is strong, we can apply the definition of $v$ as an $L^{p}$-viscosity supersolution with $u$ as a test function; we also use that $u^{-} \leq v^{-}+(u-v)^{-}$, monotonicity and subadditivity of the modulus. Then, by ABP we have that $w \leq 0$ in $\Omega$. Analogously, from the definition of subsolution of $v$, we obtain $w \geq 0$ in $\Omega$, and so $w \equiv 0$ in $\Omega$.

The approximation procedure in the above proof cannot be used to extend theorem 3.4 for unbounded $b$ and $d$, since in this case we do not have uniqueness results to infer that the limiting function is the same as the one we had started with. However, knowing a priori that the solution is strong, we 
can obtain $W^{2, p}$ a priori estimates in the general case, as stated in lemma 3.6. We give a short proof for such Nagumo's lemma in the sequel.

Proof of Lemma 3.6. Note that, in particular, $u \in C^{1, \alpha}(\bar{\Omega})$ and satisfies $F\left(x, 0,0, D^{2} u\right)=g(x)$ a.e. in $\Omega$, where

$$
g(x):=f(x)-F\left(x, u, D u, D^{2} u\right)+F\left(x, 0,0, D^{2} u\right) \in L^{p}(\Omega),
$$

since $\left|F\left(x, u, D u, D^{2} u\right)-F\left(x, 0,0, D^{2} u\right)\right| \leq b(x)|D u|+\mu\left|D u^{2}\right|+d(x) \omega(|u|) \in$ $L^{p}(\Omega)$. Now, by theorem 3.4 (for $b, d, \mu, \gamma=0$ ) and the proof there dealing with $C^{1, \alpha}$ estimates,

$$
\begin{aligned}
\|u\|_{W^{2, p}(\Omega)} \leq C & \left\{\|u\|_{L^{\infty}(\Omega)}+\|g\|_{L^{p}(\Omega)}+\|\psi\|_{W^{2, p}(\Omega)}\right\} \\
\leq C & \left\{\|u\|_{L^{\infty}(\Omega)}+\|f\|_{L^{p}(\Omega)}+\mu\|u\|_{C^{1}(\bar{\Omega})}^{2}\right. \\
& \left.+\|\psi\|_{W^{2, p}(\Omega)}+\|b\|_{L^{p}(\Omega)}\|u\|_{C^{1}(\bar{\Omega})}+\|d\|_{L^{p}(\Omega)} \omega\left(\|u\|_{\infty}\right)\right\}
\end{aligned}
$$

from where the estimate follows. 


\section{4}

\section{The Eigenvalue Problem}

This chapter is related to existence of eigenvalues for general operators with nonnegative unbounded weight. So, we leave aside for a moment the superlinear growth and "go back to the roots" of the theory, focusing on the linear growth case. As we mentioned in chapter 1, results of this type will play an important role in the multiplicity and nonexistence arguments of chapter 6 . Indeed, the lack of energy methods require techniques based on the maximum principle to overcome these limitations in the nondivergence scenario, see [66].

Throughout the chapter we are going to consider all solutions in the $L^{n}$-viscosity sense. There is no particular reason to deal with $n$ instead of $p$; we just prefer to keep the usual notation from [28]. We stress that all $L^{n}$-viscosity results are valid for $L^{p}$-viscosity ones by proposition 2.9 ; the only important assumption relies on the weight - we ask for $c \in L^{p}(\Omega), c \supsetneqq 0$, with $p>n$, as well as $b \in L_{+}^{p}(\Omega)$.

We start recalling some notations. A subset $K \subset E$ of a Banach space is an order cone if it is closed, convex, $\lambda K \subset K$ for all $\lambda \geq 0$ and $K \cap(-K)=\{0\}$. This cone induces a partial order on $E$, namely for $u, v \in E$, $u \leq v \Leftrightarrow v-u \in K$. We say that $K$ is solid if $\operatorname{int} K \neq \emptyset$. Further, a completely continuous operator, defined in $E$, is continuous and takes bounded sets into precompact ones.

Following the construction in [66], [67], we have the next Krein-Rutman theorem for general nonlinear operators; a proof is provided in section 4.1.

Theorem 4.1 (Generalized Krein-Rutman) Let $K \subset E$ be an order solid cone and let $T: K \rightarrow K$ be a completely continuous operator that is also

(i) positively 1-homogeneous, i.e. $T(\lambda u)=\lambda T u$, for all $\lambda \geq 0, u \in K$;

(ii) monotone increasing, i.e. for all $u, v \in K, u \leq v$ we have $T u \leq T v$;

(iii) strongly positive with respect to the cone, in the sense $T(K \backslash\{0\}) \subset \operatorname{int} K$.

Then $T$ has a positive eigenvalue $\alpha_{1}>0$ associated to a positive eigenfunction $w_{1} \in \operatorname{int} K$. 
Consider $\Omega \subset \mathbb{R}^{n}$ a bounded $C^{1,1}$ domain along this chapter. The application of Krein-Rutman is very standard for positive weights [66], [68]. Let us recall its use when we have a fully nonlinear operator with unbounded coefficients. About structure, we suppose (SC) with $\omega$ a Lipschitz modulus.

Consider $E:=C_{0}^{1}(\bar{\Omega})$ and the usual order solid cone of nonnegative functions over this space, i.e. $K=:\{u \in E ; u \geq 0$ in $\Omega\}$.

Let $c(x) \in L_{+}^{p}(\Omega)$ with $c>0$ in $\bar{\Omega}, p>n$. As the operator on $K$, we take $T=-F^{-1} \circ c$ in the sense that $U=T u$ iff $U$ is the unique $L^{n}$-viscosity solution of the Dirichlet problem

$$
\left\{\begin{aligned}
F\left(x, U, D U, D^{2} U\right) & =-c(x) u & & \text { in } \quad \Omega \\
U & =0 & & \text { on } \partial \Omega
\end{aligned}\right.
$$

where $F$ satisfies the following hypotheses

$$
\left\{\begin{array}{l}
\text { there exists } \theta>0 \text { such that }(\bar{H})_{\theta} \text { holds for a.e. } x_{0} \in \bar{\Omega}, \\
(\mathrm{SC}) \text { and }(S) \text { hold, } F(x, t r, t p, t X)=t F(x, r, p, X) \text { for all } t \geq 0 .
\end{array}\right.
$$

Here, hypothesis $(S)$ means the solvability in $L^{n}$-viscosity sense with data in $L^{p}$, i.e. for any $f \in L^{p}(\Omega)$, there exists a unique

$$
u \in C(\bar{\Omega}) L^{n} \text {-viscosity solution of } F[u]=f(x) \text { in } \Omega ; u=0 \text { on } \partial \Omega \text {. }
$$

Of course, Pucci's extremal operators

$$
\mathcal{L}^{ \pm}[u]:=\mathcal{M}^{ \pm}\left(D^{2} u\right) \pm b(x)|D u| \pm d(x) \omega\left(u^{\mp}\right), \quad b, d \in L_{+}^{p}(\Omega)
$$

where $\omega$ is a Lipschitz modulus, are particular examples of $F$ satisfying $(H)$. Indeed, remember that proposition 3.5 provides us with a strong solution $u \in W^{2, p}(\Omega) \subset W^{2, n}(\Omega)$, which is an $L^{n}$-viscosity solution by proposition 2.5. Furthermore, since it is unique among $L^{p}$-viscosity solutions, it is also unique among $L^{n}$-viscosity ones. Here all the coefficients can be unbounded. Observe that $(S)$ and $(\bar{H})_{\theta}$ also holds when $F$ is a uniformly continuous operator in $x$ satisfying the growth conditions in [64] (see also [69]), in this case concerning $C$-viscosity notions of solutions.

On the other hand, $(\bar{H})_{\theta},(\mathrm{SC})$ and $(S)$ are completely enough to ensure existence, uniqueness and $C^{1, \alpha}$ global regularity and estimates for the problem $\left(T_{u}\right)$ from theorem 3.1, which in turn implies that the operator $T$ is well defined and completely continuous.

Let us check the homogeneity of the operator from the homogeneity of $F$. For $\lambda=0$ it is obvious, since the problem $F\left(x, u, D u, D^{2} u\right)=0$ in $\Omega$ with 
$u=0$ on $\partial \Omega$ has only the trivial solution. Indeed, this is ensured by applying (SC) and ABP in both directions. Thus, if $\lambda>0$ and $V=T(\lambda u)$, by the homogeneity and uniqueness of $F$, we have $V / \lambda=T u$ i.e. $T(\lambda u)=\lambda T u$.

Furthermore, $T=-F^{-1} \circ c$ is strictly positive with respect to the cone, thanks to SMP and Hopf. In general, without the strict positiveness of $c$ in $\Omega$ there is no guarantee on this property, i.e., under $c \geq 0$ and $c \not \equiv 0$ in $\Omega$, we only obtain that $T(K \backslash\{0\}) \subset K$.

Notice that $T=-F^{-1} \circ c$ has an eigenvalue $\alpha_{1}>0$ associated to the positive eigenfunction $\varphi_{1}$ if and only if $\varphi_{1}$ is an $L^{n}$-viscosity solution of

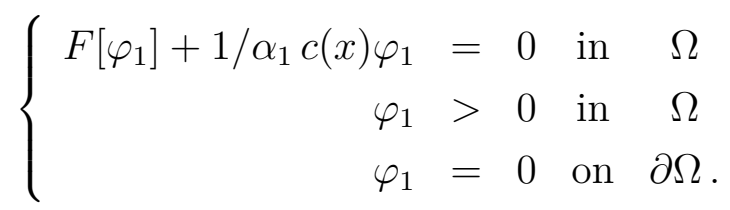

For any $c \in L^{p}(\Omega)$ with $p>n$ and $F$ satisfying $(H)$, we can define, similar to the foregoing works [70], [28], the principal weighted eigenvalues

$$
\lambda_{1}^{ \pm}=\lambda_{1}^{ \pm}(F(c), \Omega)=\sup \left\{\lambda>0 ; \Psi^{ \pm}(F(c), \Omega, \lambda) \neq \emptyset\right\}
$$

where

$$
\Psi^{ \pm}(F(c), \Omega, \lambda):=\{\psi \in C(\bar{\Omega}) ; \pm \psi>0 \text { in } \Omega, \pm(F[\psi]+\lambda c(x) \psi) \leq 0 \text { in } \Omega\}
$$

with inequalities holding in the $L^{n}$-viscosity sense. Notice that, by definition, $\lambda_{1}^{ \pm}(G(c), \Omega)=\lambda_{1}^{\mp}(F(c), \Omega)$, where $G(x, r, p, X):=-F(x, r, p, X)$.

With a simple approximation result by positive weights given by Krein-Rutman theorem as above, for $F$ satisfying $(H)$, our main result in this chapter is the following existence of eigenvalues with nonnegative weight.

Theorem 4.2 Let $\Omega \subset \mathbb{R}^{n}$ be a bounded $C^{1,1}$ domain, $c \in L^{p}(\Omega), c \supsetneqq 0$ for $p>n$ and $F$ satisfying $(H)$ for $b, d \in L_{+}^{\infty}(\Omega)$. Then $F$ has two positive weighted eigenvalues $\alpha_{1}^{ \pm}>0$ corresponding to normalized and signed eigenfunctions $\varphi_{1}^{ \pm} \in C^{1, \alpha}(\bar{\Omega})$ that satisfies

$$
\left\{\begin{array}{rllll}
F\left[\varphi_{1}^{ \pm}\right]+\alpha_{1}^{ \pm} c(x) \varphi_{1}^{ \pm} & = & \text {in } & \Omega \\
\pm \varphi_{1}^{ \pm} & > & 0 & \text { in } & \Omega \\
\varphi_{1}^{ \pm} & = & 0 & \text { on } & \partial \Omega
\end{array}\right.
$$

in the $L^{p}$-viscosity sense, with $\max _{\bar{\Omega}}\left( \pm \varphi_{1}^{ \pm}\right)=1$.

If, moreover, the operator $F$ has $W^{2, p}$ regularity of solutions (in the sense that every $u \in C(\bar{\Omega})$ which is an $L^{p}$-viscosity solution of $F[u]=f(x) \in L^{p}(\Omega)$, 
$u=0$ on $\partial \Omega$, satisfies $\left.u \in W^{2, p}(\Omega)\right)$, then $\alpha_{1}^{ \pm}=\lambda_{1}^{ \pm}$and the conclusion is valid also for $b \in L_{+}^{p}(\Omega)$.

Significant contributions on eigenvalues of continuous operators in nondivergence form in bounded domains include the fundamental work [70] for linear operators; [28] for convex fully nonlinear operators; [71] for nonlocal operators; [72], [73], [74] and the recent [75] for degenerate elliptic operators. Theorem 4.2 is a slight improvement to the general existence theory about nonconvex operators possessing first eigenvalues in [64] (see also [69]), since we are not supposing that our nonlinearity is uniformly continuous in $x$.

If, in addition, we have $W^{2, p}$ regularity of solutions, we can extend theorem 4.2 even further, allowing an unbounded first order coefficient. Eigenvalues for fully nonlinear operators with such coefficients have been previously studied, to our knowledge, only for radial operators and eigenfunctions, in [76] and [77]. As a particular case of theorem 4.2, we obtain the existence of positive eigenvalues with a nonnegative unbounded weight for the extremal Pucci's operators with unbounded coefficients.

Proposition 4.3 Let $\Omega \subset \mathbb{R}^{n}$ a bounded $C^{1,1}$ domain, $b, c \in L_{+}^{p}(\Omega), c \supsetneqq 0$, for $p>n$. Then, there exists $\varphi_{1}^{ \pm} \in W^{2, p}(\Omega)$ such that, for $\lambda_{1}^{ \pm}$defined in (4.1), we have $\lambda_{1}^{ \pm}>0$ and

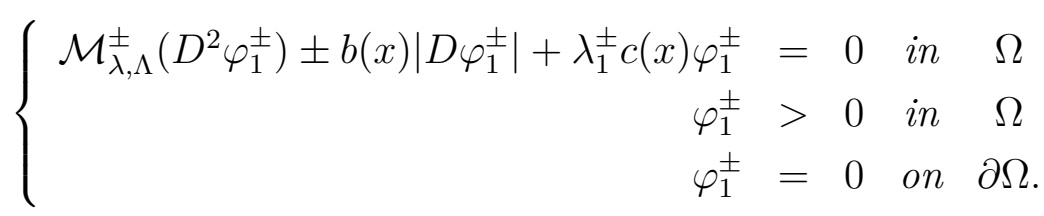

Notice that we obtain positive eigenvalues because $F$ is proper. For general existence related to nonproper operators see the script in [28] for bounded coefficients. We also stress that, without regularity assumptions on the domain, it is still possible to obtain the existence of an eigenpair, as in [28] and [70]; in such cases the eigenfunction belongs to $C_{\text {loc }}^{1, \alpha}(\Omega) \cap C(\bar{\Omega})$ by using $C^{1, \alpha}$ local regularity instead of the global one.

We start proving some auxiliary results which take into account the unboundedness of $c$.

Proposition 4.4 Let $u, v \in C(\bar{\Omega})$ be $L^{n}$-viscosity solutions of

$$
\left\{\begin{array}{rl}
F[u]+c(x) u & \geq 0 \text { in } \Omega \\
u & <0 \text { in } \Omega
\end{array},\left\{\begin{aligned}
F[v]+c(x) v & \leq 0 \text { in } \Omega \\
v & \geq 0 \text { on } \partial \Omega \\
v\left(x_{0}\right) & <0 \quad x_{0} \in \Omega
\end{aligned}\right.\right.
$$


with $F$ satisfying $(H), c \in L^{p}(\Omega), p>n$. Suppose one, $u$ or $v$, is a strong solution. Then, $u=t v$ for some $t>0$. The conclusion is the same if

$$
\left\{\begin{array}{rl}
F[u]+c(x) u & \leq 0 \text { in } \Omega \\
u & >0 \text { in } \Omega
\end{array},\left\{\begin{aligned}
F[v]+c(x) v & \geq 0 \text { in } \Omega \\
v & \leq 0 \text { on } \partial \Omega \\
v\left(x_{0}\right) & >0 \quad x_{0} \in \Omega
\end{aligned}\right.\right.
$$

For the proof of proposition 4.4, as in [64], [70], [28], we need the following consequence of $\mathrm{ABP}$, which is a maximum principle for small domains.

Lemma 4.5 Assume $F$ satisfies (SC) and $c \in L^{p}(\Omega), p>n$. Then there exists $\varepsilon_{0}>0$, depending on $n, p, \lambda, \Lambda,\|b\|_{L^{p}(\Omega)},\left\|c^{+}\right\|_{L^{p}(\Omega)}$ and $\operatorname{diam}(\Omega)$, such that if $|\Omega| \leq \varepsilon_{0}$ then any $u \in C(\bar{\Omega})$ which is an $L^{n}$-viscosity solution of

$$
\left\{\begin{aligned}
F[u]+c(x) u & \geq 0 \text { in } \Omega \\
u & \leq 0 \text { on } \partial \Omega
\end{aligned}\right.
$$

satisfies $u \leq 0$ in $\Omega$. Analogously, if $v \in C(\bar{\Omega})$ is an $L^{n}$-viscosity solution of

$$
\left\{\begin{aligned}
F[v]+c(x) v & \leq 0 \text { in } \Omega \\
v & \geq 0 \text { on } \partial \Omega
\end{aligned}\right.
$$

then we have that $v \geq 0$ in $\Omega$ provided $|\Omega| \leq \varepsilon_{0}$.

Proof. Assume $u$ satisfies (4.6). In order to obtain a contradiction, suppose that $\Omega^{+}:=\{u>0\}$ is not an empty set. By (SC), we have that $u$ is an $L^{n}$-viscosity solution of $\mathcal{L}^{+}[u] \geq \mathcal{L}^{+}[u]-c^{-}(x) u \geq-c^{+}(x) u$ in $\Omega^{+}$. So, ABP gives us that

$$
\sup _{\Omega^{+}} u \leq C_{1} \operatorname{diam}(\Omega)\left\|c^{+}\right\|_{L^{n}(\Omega)} \sup _{\Omega^{+}} u \leq C_{1} \operatorname{diam}(\Omega)|\Omega|^{1-\frac{n}{p}}\left\|c^{+}\right\|_{L^{p}(\Omega)} \sup _{\Omega^{+}} u .
$$

Then we choose $\epsilon_{0}>0$ such that $C_{1} \operatorname{diam}(\Omega) \varepsilon_{0}^{1-\frac{n}{p}}\left\|c^{+}\right\|_{L^{p}(\Omega)} \leq 1 / 2$ to obtain a contradiction. If $v$ is a supersolution the proof is similar, by using ABP in the opposite direction.

Remark 4.6 We emphasize once more that, for equations satisfying (SC), the notions of $L^{p}$ and $L^{n}$ viscosity solutions are equivalent, by proposition 2.9. Notice that the addition of the term $c(x) u$ does not change the proof there, since the behavior of the zero order term is irrelevant to that proof.

However, we can give a more direct proof of proposition 4.4 for $L^{p}$-viscosity solutions. As a matter of fact, it is just a question of using ABP 
(for $L^{p}$-visc.) with the $L^{n}$-norm of $f$ in the proof above (lemma 4.5); the proof of proposition 4.4 below is also unchangeable, just reading $L^{p}$ instead of $L^{n}$.

We are going to use this result in chapter 6 with the operator $F$ being $\mathcal{L}^{-}[u]+c(x) u$, in the case of inequalities (4.5).

Proof of Proposition 4.4. We are going to prove the first case, since the second is analogous. Let $u, v$ be $L^{n}$-viscosity solutions of (4.4). Say both are strong, otherwise just use test functions for one of them and read all inequalities below in the $L^{n}$-viscosity sense. Set $z_{t}:=t u-v$ for $t>0$. Then, using 1-homogeneity and (SC), we have that $z_{t}$ is a solution of

$$
\begin{aligned}
\mathcal{L}^{+}\left[z_{t}\right] & +d(x) \omega\left(\left(-z_{t}\right)^{+}\right)+c(x) z_{t} \geq F[t u]-F[v]+c(x) z_{t} \\
& =t\{F[u]+c(x) u\}-\{F[v]+c(x) v\} \geq 0 \text { in } \Omega .
\end{aligned}
$$

Let $K$ be a compact subset of $\Omega$ such that $x_{0} \in K$ and MP lemma 4.5 holds for $\Omega \backslash K$. Further, let $t_{0}>0$ be large enough such that $z_{t_{0}} \leq 0$ in $K$. In fact, this $t_{0}$ can be taken as $\min _{K} v / \max _{K} u>0$, since $u<0$ in $K$ and $\min _{K} v \leq v\left(x_{0}\right)<0$. Then, since $z_{t_{0}} \leq 0$ in $\partial(\Omega \backslash K) \subset \partial \Omega \cup \partial K$, we obtain from lemma 4.5 that $z_{t_{0}} \leq 0$ in $\Omega \backslash K$ and so in $\Omega$.

Define

$$
\tau:=\inf \left\{t>0 ; z_{t} \leq 0 \text { in } \Omega\right\} \geq t_{0}>0
$$

By (SC), $z_{\tau}$ is a solution of $\mathcal{L}^{-}\left[-z_{\tau}\right]+\{c(x)-d(x) \omega(1)\}\left(-z_{\tau}\right) \leq 0$ in $\Omega$, with $z_{\tau} \leq 0$ in $\Omega$. Hence, by SMP, we have either $z_{\tau} \equiv 0$ or $z_{\tau}<0$ in $\Omega$. In the first case we are done. Suppose, then, $z_{\tau}<0$ in $\Omega$ in order to obtain a contradiction.

Next we choose some $\varepsilon>0$ such that $z_{\tau-\varepsilon}<0$ in $K$. Indeed, we can take, for example, $\varepsilon=\min \left\{-\min _{K} z_{\tau} /\left(2\|u\|_{L^{\infty}(K)}\right), \tau / 2\right\}$, which implies

$$
z_{\tau-\varepsilon}=z_{\tau}-\varepsilon u \leq \min _{K} z_{\tau}+\varepsilon\|u\|_{L^{\infty}(K)}<0 \text { in } K,
$$

as in [78]. In particular, $z_{t}$ satisfies (4.7) for $t=\tau-\varepsilon>0$. Thus, $z_{\tau-\varepsilon} \leq 0$ by MP in the set $\Omega \backslash K$. By SMP, $z_{\tau-\varepsilon}<0$ in $\Omega$, which contradicts the definition of $\tau$ being an infimum.

The next result was first introduced in [70] and extended in [28] to nonlinear operators. We show below that, when we add an unbounded weight $c$, all we need is its positiveness on a subset of positive measure in order to obtain a bound from above on $\lambda_{1}$; we also make some remarks about the constant.

Lemma 4.7 Suppose $(H)$ with $b, d \in L_{+}^{\infty}(\Omega)$. If $c \geq \delta>0$ a.e. in $B_{R} \subset \subset \Omega$, for $R \leq 1$, then

$$
\lambda_{1}^{ \pm}(F(c), \Omega) \leq \frac{C_{0}}{\delta R^{2}}
$$


where $C_{0}$ depends on $n, \lambda, \Lambda, R,\|b\|_{L^{\infty}(\Omega)}$ and $\omega(1)\|d\|_{L^{\infty}(\Omega)}$.

If, moreover, $F$ has no term of order zero (i.e. $d$ or $\omega$ is equal to zero), then $R$ can be any positive number. On the other hand, if $b \equiv 0$, then the constant $C_{0}$ does not depend on $R$.

Proof. Observe that $\lambda_{1}^{ \pm}(F(c), \Omega) \leq \lambda_{1}^{ \pm}\left(F(c), B_{R}\right)$ by definition.

As in [70], [28], consider the radial function $\sigma(x)=-\left(R^{2}-|x|^{2}\right)^{2}<0$ in $B_{R}$. Let us treat the $\lambda_{1}^{-}$case; for $\lambda_{1}^{+}$it is just a question of looking at $-\sigma$.

Suppose, in order to obtain a contradiction, that there exists some $\lambda>\frac{C_{0}}{\delta R^{2}}$ such that $\Psi^{-}(F(c), \Omega, \lambda) \neq \emptyset$, i.e. let $\psi \in C(\bar{\Omega})$ be a negative $L^{n}$-viscosity solution of $F[\psi]+\lambda c(x) \psi \geq 0$ in $\Omega$. Hence $\psi$ is also an $L^{n}$-viscosity solution of $F[\psi]+\frac{C_{0}}{\delta R^{2}} c(x) \psi \geq 0$ in $B_{R}$.

Claim 4.8 We have $F[\sigma]+\frac{C_{0}}{\delta R^{2}} c(x) \sigma \leq 0$ a.e. in $B_{R}$.

Proof of Claim 4.8. Notice that

$$
D \sigma(x)=4\left(R^{2}-|x|^{2}\right) x, \quad D^{2} \sigma(x)=4\left(R^{2}-|x|^{2}\right) I-8 x \otimes x .
$$

Say, for example, $0 \leq b(x) \leq \gamma$ and $0 \leq d(x) \leq \eta$ a.e., thus

$$
\begin{aligned}
F[\sigma] & \leq \mathcal{M}^{+}\left(D^{2} \sigma\right)+\gamma|D \sigma|-\theta A \sigma \\
& \leq 4\left(R^{2}-|x|^{2}\right) \mathcal{M}^{+}(I)-8 \mathcal{M}^{-}(x \otimes x)+4 \gamma\left(R^{2}-|x|^{2}\right)|x|-\eta \omega(1) \sigma(x)
\end{aligned}
$$

and therefore

$$
\frac{F[\sigma]}{\sigma} \geq \frac{8 \lambda|x|^{2}}{\left(R^{2}-|x|^{2}\right)^{2}}-\frac{4 n \Lambda}{R^{2}-|x|^{2}}-\frac{4 \gamma R}{R^{2}-|x|^{2}}-\eta \omega(1) \text { a.e. in } B_{R} .
$$

The first term is always nonnegative, but it is extremely important to control the middle terms when $x$ is near from $R$. Explicitly, we have that

$$
\frac{8 \lambda|x|^{2}}{\left(R^{2}-|x|^{2}\right)^{2}} \geq \frac{4 n \Lambda}{R^{2}-|x|^{2}}+\frac{4 \gamma R}{R^{2}-|x|^{2}} \Leftrightarrow|x|^{2} \geq \alpha R^{2}
$$

for $\alpha=\frac{n \Lambda+\gamma R}{2 \lambda+n \Lambda+\gamma R} \in(0,1)$. Then we separate the analysis in two cases.

(a) $|x|^{2} \geq \alpha R^{2}$ : It follows that $F[\sigma] / \sigma \geq-\eta \omega(1) \geq-\eta \omega(1) c(x) /\left(\delta R^{2}\right)$ from construction;

(b) $|x|^{2} \leq \alpha R^{2}$ : In this case we use that the first term is nonnegative and so $F[\sigma] / \sigma \geq-4(n \Lambda+\gamma R) /\left((1-\alpha) R^{2}\right)-\eta \omega(1) \geq-C_{0} c(x) /\left(\delta R^{2}\right)$.

If $\eta$ or $\omega$ is equal to zero, then we do not need to use $R \leq 1$ in the above. Furthermore, if $\gamma=0, C_{0}$ does not depend on $R$. 
Now we apply proposition 4.4, since $\sigma \in C^{2}\left(\bar{B}_{R}\right)$, obtaining that $\psi=t \sigma$, for some $t>0$. However, this is not possible, since $\psi<0$ on $\partial B_{R} \subset \Omega$ while $\sigma=0$ on $\partial B_{R}$.

Moving to the last statement in theorem 4.2, we first prove an eigenvalue bound that takes into account an unbounded $b$, when the weight is a continuous and positive function in $\bar{\Omega}$. Note that, in this case, theorem 4.1 gives us a pair $\alpha_{1}>0$ and $\varphi_{1} \in C^{1}(\bar{\Omega})$ such that

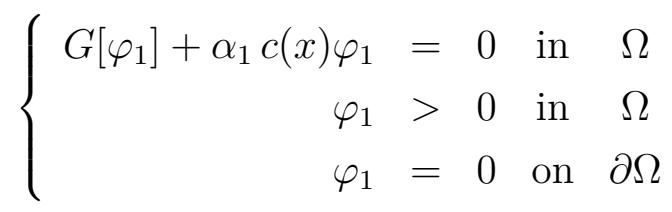

in the $L^{n}$-viscosity sense, with $\max _{\bar{\Omega}} \varphi_{1}=1$ and

$$
0<\alpha_{1} \leq \lambda_{1}^{+}(G(c), \Omega)=\lambda_{1}^{-}(F(c), \Omega)
$$

The following proposition is a delicate point in our construction of an eigenpair. It states that $\alpha_{1}$ in (4.8) is bounded, and this does not seem to be a consequence of the usual methods for bounding a first eigenvalue, such as the one in lemma 4.7. Instead, we use the classical blow-up method [79] of Gidas and Spruck.

Proposition 4.9 Assume $c \in C(\bar{\Omega})$ with $c>0$ in $\bar{\Omega}$, and $G$ satisfying $(H)$ for $b \in L_{+}^{p}(\Omega)$ and $d \in L_{+}^{\infty}(\Omega)$. Let $\alpha_{1}$ and $\varphi_{1}$ as in (4.8). Then $\alpha_{1} \leq C$, for a constant $C=C\left(n, \lambda, \Lambda, \Omega,\|b\|_{L^{p}(\Omega)}, \omega(1)\|d\|_{L^{\infty}(\Omega)}\right)$.

Proof. If the conclusion is not true, then exists a sequence $b_{k} \in L_{+}^{\infty}(\Omega)$, with $\left\|b_{k}\right\|_{L^{p}(\Omega)} \leq C,\left\|b_{k}\right\|_{L^{\infty}(\Omega)} \rightarrow+\infty$ and the respective eigenvalue problem

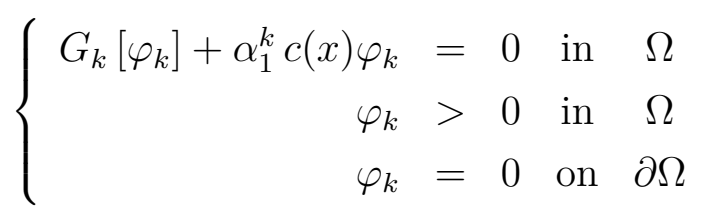

in the $L^{n}$-viscosity sense, with $\max _{\bar{\Omega}} \varphi_{k}=1$ for all $k \in \mathbb{N}$ and $\alpha_{1}^{k} \rightarrow+\infty$ as $k \rightarrow+\infty$, where $G_{k}$ is a fully nonlinear operator satisfying $(H)_{k}$, i.e. $(H)$ for $b_{k}$ and $d_{k}$. Say $d_{k} \leq \eta$ and $\max _{\bar{\Omega}} \varphi_{k}=\varphi_{k}\left(x_{0}^{k}\right)$ for $x_{0}^{k} \in \Omega$. Then, $x_{0}^{k} \rightarrow x_{0} \in \bar{\Omega}$ as $k \rightarrow+\infty$, up to a subsequence.

Case 1: $x_{0} \in \Omega$. Let $2 \rho=\operatorname{dist}\left(x_{0}, \partial \Omega\right)>0$ and notice that $x_{0}^{k} \in B_{\rho}\left(x_{0}\right)$ for all $k \geq k_{0}$. Set $r_{k}=\left(\alpha_{1}^{k}\right)^{-1 / 2}$ and define $\psi_{k}(x)=\varphi_{k}\left(x_{0}^{k}+r_{k} x\right)$. Thus, $\psi_{k}$ is an $L^{n}$ (so $\left.L^{p}\right)$ viscosity solution of

$$
\widetilde{G}_{k}\left(x, \psi_{k}, D \psi_{k}, D^{2} \psi_{k}\right)+c_{k}(x) \psi_{k}(x)=0 \quad \text { in } \quad \widetilde{B}_{k}:=B_{\rho / r_{k}}(0)
$$


where $c_{k}(x):=c\left(x_{0}^{k}+r_{k} x\right), \widetilde{G}_{k}(x, r, p, X):=r_{k}^{2} G_{k}\left(x_{0}^{k}+r_{k} x, r, p / r_{k}, X / r_{k}^{2}\right)$ satisfies $(\widetilde{H})_{k}$, i.e. $(H)$ for $\widetilde{b_{k}}$ and $\eta_{k}$, where $\widetilde{b}_{k}(x):=r_{k} b_{k}\left(x_{0}^{k}+r_{k} x\right)$ and $\eta_{k}=r_{k}^{2} \eta$. Notice that $b_{k}$ and $\eta_{k}$ converge locally to zero in $L^{p}\left(\widetilde{B}_{k}\right)$ as $k \rightarrow+\infty$, since $p>n$.

Furthermore, $\sup _{\widetilde{B}_{k}} \psi_{k}=\psi_{k}(0)=1$ for all $k \in \mathbb{N}$ and $B_{R}(0) \subset \subset \widetilde{B}_{k}$ for large $k$, for any fixed $R>0$. By theorem 3.1 we have that $\psi_{k}$ is locally in $C^{1, \alpha}$ and satisfies the estimate

$$
\left\|\psi_{k}\right\|_{C^{1, \alpha}\left(\bar{B}_{R}(0)\right)} \leq C_{k}\left\|\psi_{k}\right\|_{L^{\infty}\left(\widetilde{B_{k}}\right)} \leq C
$$

since $\psi_{k}$ attains its maximum at 0 and $C_{k}$ only depends on the $L^{p}$-norm of the coefficients $b_{k}$ and $c_{k}$, which are uniformly bounded in there. Hence, by compact inclusion we have that there exists $\psi \in C^{1}\left(\bar{B}_{R}(0)\right)$ such that $\psi_{k} \rightarrow \psi$ as $k \rightarrow+\infty$, up to a subsequence. Performing the same argument for each ball $B_{R}(0)$, for every $R>0$, we obtain in particular that $\psi_{k} \rightarrow \psi$ in $L_{\text {loc }}^{\infty}\left(\mathbb{R}^{n}\right)$, by using the uniqueness of the limit for $\psi_{k}$ in the smaller balls.

Using stability (proposition 2.12 together with observation 2.13) and the continuity of $c$, we have that $\psi$ is an $L^{p}$-viscosity solution of $J\left(x, D^{2} \psi\right)+$ $c\left(x_{0}\right) \psi=0$ in $\mathbb{R}^{n}$ for some measurable operator $J$ still satisfying $(H)$ with coefficients of zero and first order term, $d$ and $b$, equal to zero. Also, $\psi(0)=1$ and $\psi>0$ in $\mathbb{R}^{n}$ by SMP. This implies that $1 \leq \lambda_{1}^{+}\left(J\left(c\left(x_{0}\right)\right), B_{R}\right) \leq \frac{C_{0}}{c\left(x_{0}\right) R^{2}}$ for all $R>0$, which gives a contradiction when we take $R \rightarrow+\infty$.

Case 2: $x_{0} \in \partial \Omega$. By passing to new coordinates, that come from the smoothness property of the domain $\partial \Omega \in C^{1,1}$, we can suppose that $\partial \Omega \subset\left\{x_{n}=0\right\}$ and $\Omega \subset\left\{x_{n}>0\right\}$.

Set $\rho_{k}=\operatorname{dist}\left(x_{0}^{k}, \partial \Omega\right)=x_{0}^{k} \cdot e_{n}=x_{0, n}^{k}$, where $e_{n}=(0, \ldots, 0,1)$ and $x_{0}^{k}=$ $\left(x_{0,1}^{k}, \ldots, x_{0, n}^{k}\right)$. Analogously, consider $\psi_{k}(y)$ in $y \in B_{\rho_{k} / r_{k}}(0)$ and the respective equation $\widetilde{G}_{k}$ as in case 1 . Thus, for $x, y$ satisfying $r_{k} y=x-x_{0}^{k}$, we have that the set $\left\{x_{n}>0\right\}$ corresponds to $A_{k}:=\left\{y_{n}=\left(x-x_{0}^{k}\right) \cdot e_{n} / r_{k}>-\rho_{k} / r_{k}\right\}$. So we need to analyze the behavior of the set $A_{k}$ when we pass to limits as $k \rightarrow+\infty$.

We first claim that $\rho_{k} / r_{k}$ is bounded below by a constant $C_{1}>0$, which means that $A_{k}$ does not converge to $\left\{y_{n}>0\right\}$. This is an easy consequence of our $C^{1, \alpha}$ boundary regularity and estimates in a half ball (theorem 3.14), applied to $\psi_{k}$ and $\widetilde{G}_{k}$. Indeed, since $\left\|D \psi_{k}\right\|_{L^{\infty}\left(B_{r}^{+}(0)\right)} \leq C, r>0$ fixed, then

$$
1=\left|\varphi\left(x_{0}^{k}\right)-\varphi\left(\bar{x}_{0}^{k}\right)\right|=\left|\psi_{k}(0,0)-\psi_{k}\left(0,-\rho_{k} / r_{k}\right)\right| \leq C \rho_{k} / r_{k},
$$

where $\bar{x}_{0}^{k}=\left(x_{0,1}^{k}, \ldots, x_{0, n-1}^{k}, 0\right) \in \partial \Omega$, from where we obtain the desired bound.

Next observe that we have two possibilities about the fraction $\rho_{k} / r_{k}$, either it converges to $+\infty$ or it is uniformly bounded. In the first one, $A_{k} \rightarrow \mathbb{R}^{n}$ 
and we finish as in case 1 . In the second, $A_{k} \rightarrow\left\{y_{n}>\varrho\right\}, \varrho \in(0,+\infty)$, by taking a subsequence. The proof carries on as in the case 1 , since we have a smooth domain which contains a ball with radius $R=\left(2 C_{0} / c\left(x_{0}\right)\right)^{1 / 2}$; this gives the final contradiction.

Lemma 4.10 Let $c \in L^{p}(\Omega), c \geq \delta$ in $B_{R}$ for some $B_{R} \subset \subset \Omega$ and $F$ satisfying hypothesis $(H)$, then

$$
\lambda_{1}^{ \pm}(F(c), \Omega) \leq \frac{\lambda_{1}^{ \pm}\left(F(1), B_{R}\right)}{\delta}
$$

Proof. Let us prove the $\lambda_{1}^{+}$case; for $\lambda_{1}^{-}$we use $G$ instead of $F$. We already know that both quantities are nonnegative, by the properness of the operator $F$. Hence, it is enough to verify that $\mathcal{A} \cap\{\lambda \geq 0\} \subset \mathcal{B} / \delta \cap\{\lambda \geq 0\}$, where

$$
\lambda_{1}^{+}(F(c), \Omega)=\sup _{\mathcal{A}} \lambda=\sup _{\mathcal{A} \cap\{\lambda \geq 0\}} \lambda, \lambda_{1}^{+}\left(F(1), B_{R}\right)=\sup _{\mathcal{B}} \lambda=\sup _{\mathcal{B} \cap\{\lambda \geq 0\}} \lambda
$$

as defined before. Let $\lambda \in \mathcal{A} \cap\{\lambda \geq 0\}$, then there exists $\psi \in C(\bar{\Omega})$ a nonnegative $L^{n}$-viscosity solution of $F[\psi]+c(x) \lambda \psi \leq 0$ in $\Omega$. Then, $\psi$ is also a nonnegative $L^{n}$-viscosity solution of $F[\psi]+\delta \lambda \psi \leq 0$ in $B_{R}$, so $\delta \lambda \in \mathcal{B}$.

Proof of Theorem 4.2. First, from the fact that $c>0$ in a set of positive measure, there exists $\delta>0$ such that $\{c \geq \delta\}$ is a nontrivial set. In fact, if this was not true, i.e. if $|\{c \geq \delta\}|=0$ for all $\delta$, then $\{c>0\}=\bigcup_{\delta>0}\{c \geq \delta\}$ would have measure zero, as the union of such sets, contradicting the hypothesis. Namely, then, $c \geq \delta>0$ a.e. in some ball $B_{R} \subset \subset \Omega$.

Let us prove the $\lambda_{1}^{-}$case, applying Krein-Rutman results to $G$; for $\lambda_{1}^{+}$ replace $G$ by $F$. Let $\varepsilon \in(0,1)$ and define $c_{\varepsilon}:=c+\varepsilon>0$ in $\Omega$, for all $\varepsilon$. From theorem 4.1 , we obtain the existence of pairs $\alpha_{1}^{\varepsilon}>0$ and $\varphi_{1}^{\varepsilon} \in C^{1}(\bar{\Omega})$ such that



with $\max _{\bar{\Omega}} \varphi_{1}^{\varepsilon}=1$ for all $\varepsilon \in(0,1)$. Then,

$$
0<\alpha_{1}^{\varepsilon} \leq \lambda_{1}^{+}\left(G\left(c_{\varepsilon}\right), \Omega\right)=\lambda_{1}^{-}\left(F\left(c_{\varepsilon}\right), \Omega\right) \leq \frac{C_{0}}{\delta R^{2}} \text { for all } \varepsilon \in(0,1)
$$

Next, $\alpha_{1}^{\varepsilon} \rightarrow \alpha_{1} \in\left[0, C_{0} / \delta R^{2}\right]$ up to a subsequence. Then we apply $C^{1, \alpha}$ global regularity and estimates (theorem 3.1) in the case $\mu=0$ (recall again that $L^{n}$-viscosity solutions are $L^{p}$-viscosity for $p>n$ ), by considering 
$\alpha_{1}^{\varepsilon} c_{\varepsilon}(x) \varphi_{1}^{\varepsilon} \in L^{p}(\Omega)$ as the right hand side, from where

$$
\begin{aligned}
\left\|\varphi_{1}^{\varepsilon}\right\|_{C^{1, \alpha}(\bar{\Omega})} & \leq C\left\{\left\|\varphi_{1}^{\varepsilon}\right\|_{L^{\infty}(\Omega)}+\alpha_{1}^{\varepsilon}\left\|c_{\varepsilon}\right\|_{L^{p}(\Omega)}\left\|\varphi_{1}^{\varepsilon}\right\|_{\infty}\right\} \\
& \left.\leq C C_{1}\left(\|c\|_{L^{p}(\Omega)}+1\right)\right\} \leq C .
\end{aligned}
$$

Hence the compact inclusion $C^{1, \alpha}(\bar{\Omega}) \subset C^{1}(\bar{\Omega})$ yields $\varphi_{1}^{\varepsilon} \rightarrow \varphi_{1} \in C^{1}(\bar{\Omega})$, up to a subsequence. Of course that this implies $\max _{\bar{\Omega}} \varphi_{1}=1, \varphi_{1} \geq 0$ in $\Omega$ and $\varphi_{1}=0$ on $\partial \Omega$.

Since $c_{\varepsilon} \rightarrow c$ in $L^{p}(\Omega)$ as $\varepsilon \rightarrow 0$, by proposition 2.12 we have that $\varphi_{1}$ is an $L^{p}$-viscosity solution of $G\left[\varphi_{1}\right]+\alpha_{1} c(x) \varphi_{1}=0$ in $\Omega$, which allows us to apply $C^{1, \alpha}$ regularity again to obtain that $\varphi_{1} \in C^{1, \alpha}(\bar{\Omega})$.

Using now that $\varphi_{1}$ is a nonnegative $L^{p}$-viscosity solution of

$$
\mathcal{L}^{-}\left[\varphi_{1}\right]-\left(d(x) \omega(1)-\alpha_{1} c(x)\right) \varphi_{1} \leq 0 \text { in } \Omega
$$

together with SMP, we have that $\varphi_{1}>0$ in $\Omega$, since $\max _{\bar{\Omega}} \varphi_{1}=1$. Moreover, we must have $\alpha_{1}>0$, because the case $\alpha_{1}=0$ would imply that $\varphi_{1}$ is an $L^{p}$-viscosity solution of $\mathcal{L}^{+}\left[\varphi_{1}\right] \geq 0$ in $\Omega \cap\left\{\varphi_{1}>0\right\}$ (since $F$ is proper, and so $G$ ) which, in turn, would give us $\varphi_{1} \leq 0$ in $\Omega$, by ABP. Thus, the existence property is completed.

In order to conclude that, under $W^{2, p}$ regularity assumptions over $F$, the $\alpha_{1}$ obtained is equal to $\lambda_{1}^{-}=\lambda_{1}^{-}(F(c), \Omega)$, related to the eigenfunction $\varphi_{1}^{-}=\varphi_{1}^{-}(F(c), \Omega)=-\varphi_{1}<0$ in $\Omega$, we have to work a little bit more, as in proposition 4.7 in [28].

We already have $\alpha_{1} \leq \lambda_{1}^{-}$. Suppose by contradiction that $\alpha_{1}<\lambda_{1}^{-}$. By definition of $\lambda_{1}^{-}$as a supremum, we know that $\alpha_{1}$ cannot be an upper bound, that is, there exists $\lambda>0$ such that $\Psi^{-}(F(c), \Omega, \lambda) \neq \emptyset$ and $\alpha_{1}<\lambda \leq \lambda_{1}^{-}$. Then we obtain $\psi \in C(\bar{\Omega})$ such that $F[\psi]+\lambda c(x) \psi \geq 0$ in $\Omega$ in the $L^{n}$-viscosity sense, with $\psi<0$ in $\Omega$. Now, since $c \supsetneqq 0$, we have $c(x)\left(\lambda-\alpha_{1}\right) \supsetneqq 0$. Therefore $\psi$ is a negative $L^{n}$-viscosity solution of

$$
F[\psi]+\alpha_{1} c(x) \psi \supsetneqq F[\psi]+\lambda c(x) \psi \geq 0 \text { in } \Omega .
$$

Then, under $W^{2, p}$ regularity, we have that $\varphi_{1}^{-} \in W^{2, p}(\Omega) \subset W^{2, n}(\Omega)$ is a strong solution of

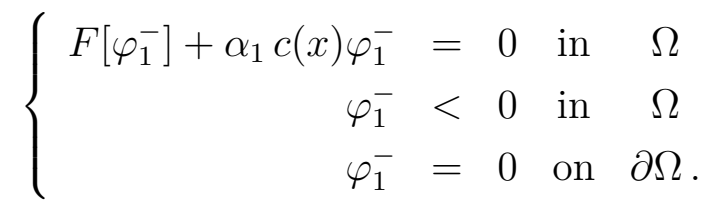


Applying proposition 4.4 we obtain that $\psi=t \varphi_{1}^{-}$for some $t>0$; but this contradicts the strict inequality in (4.12). Thus, we must have $\alpha_{1}=\lambda_{1}^{-}$. The case of $\lambda_{1}^{+}$is completely analogous, by reversing the inequalities.

From this last paragraph, under $W^{2, p}$ regularity of the solutions, the only possibility for $\alpha_{1}$ is to coincide with $\lambda_{1}$. Therefore, by using proposition 4.9 (with $c \equiv 1$ ) and lemma 4.10, we obtain that $\lambda_{1}^{-}\left(F\left(c_{\varepsilon}\right), \Omega\right) \leq C_{1} / \delta$, for all $\varepsilon \in(0,1)$, where $C_{1}$ depends on $n, \lambda, \Lambda, R,\|b\|_{L^{p}(\Omega)}$ and $\omega(1)\|d\|_{L^{\infty}(\Omega)}$. Thus, we carry on this bound on $\lambda_{1}$, instead of (4.11), in the limiting procedure, in order to get the desired existence result for $b \in L_{+}^{p}(\Omega)$.

\section{1}

\section{The Krein-Rutman Theorem}

In this section we prove theorem 4.1. We start recalling the following "Rabinowitz type" result about existence of solutions via Leray-Schauder degree for perturbations of the identity, i.e. for operators in the form $I-T$, where $T$ is completely continuous. We state it as in theorem 3.3 in [66] (see there for a proof); see also theorem 3.5.3 in [80] and theorem 4.11 in [81]. For this, consider the fixed point problem

$$
x=T(\lambda, x) .
$$

In the following we say continuum to mean a closed and connected set.

Proposition 4.11 Let $T: \mathbb{R} \times E \rightarrow E$ be such that $T_{\lambda}=T(\lambda, \cdot): E \rightarrow E$ is completely continuous for all $\lambda \in \mathbb{R}$ and $T(\lambda, x)$ is continuous in $\lambda$ uniformly with respect to $x$ in balls of $E$. Let $\left(\lambda_{0}, x_{0}\right)$ be a solution of (4.13).

Suppose that $\mathcal{U} \subset E$ is an open bounded set such that $x_{0} \in \mathcal{U}$ and there is no other solution $x$ of $x=T\left(\lambda_{0}, x\right)$ in $\overline{\mathcal{U}}$, with $\operatorname{deg}\left(I-T_{\lambda_{0}}, \mathcal{U}, 0\right) \neq 0$.

Then there exist two continuums, $\mathcal{C}^{-} \subset\left(-\infty, \lambda_{0}\right] \times E$ and $\mathcal{C}^{+} \subset$ $\left[\lambda_{0},+\infty\right) \times E$, of solutions of (4.13) with $\left(\lambda_{0}, x_{0}\right) \in \mathcal{C}^{+} \cap \mathcal{C}^{-}$and such that one of the following alternatives holds

(i) $\mathcal{C}^{+}$is unbounded;

(ii) $\mathcal{C}^{+} \cap\left(\left\{\lambda_{0}\right\} \times(E \backslash \overline{\mathcal{U}})\right) \neq \emptyset$, i.e. $\mathcal{C}^{+}$bends back to $\lambda_{0}$.

The same alternatives hold for $\mathcal{C}^{-}$.

In particular, if $T(0, x)=0$ for all $x \in E$, then there exist two unbounded continuums of solutions meeting each other at $(0,0)$.

With this tool at hand, we give a proof for the generalized Krein-Rutman theorem in the sequel. 
Proof of Theorem 4.1. Fix $u_{0} \in K \backslash\{0\}$. We first claim that there exists $m>0$ such that $m T u_{0} \geq u_{0}$. If this was not true, i.e. if for all $m>0$, $T u_{0}-u_{0} / m \notin K$, by taking the limit as $m \rightarrow+\infty$ we would have $T u_{0} \notin \operatorname{int} K$, which contradicts item (iii). Now, for each $\varepsilon>0$, define the map

$$
\begin{aligned}
T_{\varepsilon}:[0,+\infty) \times K & \rightarrow K \\
(\lambda, u) & \mapsto \lambda T u+\varepsilon \lambda T u_{0}
\end{aligned}
$$

which is completely continuous, continuous in $\lambda$ and satisfies $T_{\varepsilon}(0, \cdot) \equiv 0$. By theorem 4.11, there exists an unbounded connected component $C_{\varepsilon}$ of solutions of $T_{\varepsilon}(\lambda, u)=u$. Let us see that $C_{\varepsilon}$ is bounded in the $\lambda$-direction.

Claim $4.12 u \geq \varepsilon\left(\frac{\lambda}{m}\right)^{k} u_{0}$, for all $k \in \mathbb{N}$ and all $(\lambda, u) \in C_{\varepsilon}$.

Notice that this claim implies that $\lambda \leq m$. If not, if there would exists $(\lambda, u) \in C_{\varepsilon}$ with $\lambda>m$, we would have $u_{0} \leq \frac{1}{\varepsilon}\left(\frac{m}{\lambda}\right)^{k} u \rightarrow 0$ as $k \rightarrow+\infty$ in the norm of $E$, yielding $u_{0} \notin K$, a contradiction.

Proof. Let $(\lambda, u) \in C_{\varepsilon}$, then $u=\lambda T u+\varepsilon \lambda T u_{0} \geq \varepsilon \lambda T u_{0} \geq \varepsilon \frac{\lambda}{m} u_{0}$ and the claim is proved for $k=1$. Moreover, using that that $T$ is increasing and positively 1-homogeneous, $T u \geq T\left(\varepsilon \frac{\lambda}{m} u_{0}\right)=\varepsilon \frac{\lambda}{M} T u_{0} \geq \varepsilon \frac{\lambda}{m^{2}} u_{0}$. Since $u \geq \lambda T u$, it follows that $u \geq \varepsilon\left(\frac{\lambda}{m}\right)^{2} u_{0}$. By iteration we obtain the conclusion for all $k \in \mathbb{N}$.

Since $C_{\varepsilon}$ is unbounded, it has to be unbounded in the $K$-direction, that is, there must exist a sequence of solutions $(\lambda, u) \in C_{\varepsilon}$ with $\|u\|_{E} \rightarrow+\infty$ for each $\varepsilon>0$; in particular, there exists $\left(\lambda_{\varepsilon}, u_{\varepsilon}\right) \in C_{\varepsilon}$ with $\left\|u_{\varepsilon}\right\|_{E}=1$. Then, by taking the limit as $\varepsilon \rightarrow 0$ we can obtain the desired pair $\left(\lambda_{1}, u_{1}\right)$ with $\lambda_{1} \in[0, M]$ and $\left\|u_{1}\right\|_{E}=1$ such that $u_{1}=\lambda_{1} T u_{1}$.

Indeed, since $T$ takes bounded sets into precompact ones, there exists some $v \in K$ such that $T u_{\varepsilon} \rightarrow v$ in $E$, for a subsequence of $\varepsilon \rightarrow 0$. Then there exists the limit of $u_{\varepsilon}=\lambda_{\varepsilon} T u_{\varepsilon}+\varepsilon \lambda_{\varepsilon} T u_{\varepsilon} \rightarrow \lambda_{1} v=: u_{1}$ for some $\lambda_{1} \in[0, m]$, up to a subsequence of $\varepsilon \rightarrow 0$. Finally, $T$ continuous yields $v=T u_{1}$.

We notice that $\lambda_{1}$ must be positive; otherwise we would have $u_{1} \equiv 0$, contradicting $\left\|u_{1}\right\|_{E}=1$. Furthermore, since $u_{1} \in K \backslash\{0\}$, it follows that $T u_{1}$ belongs to the interior of $K$, and so does $u_{1}=\lambda_{1} T u_{1}$. 


\section{A Priori Bounds and Multiplicity Results}

In this chapter we consider a family of fully nonlinear uniformly elliptic problems of the following form

$$
\left\{\begin{aligned}
-F\left(x, u, D u, D^{2} u\right) & =\lambda c(x) u+\langle M(x) D u, D u\rangle+h(x) & & \text { in } \quad \Omega \\
u & =0 & & \text { on } \partial \Omega
\end{aligned}\right.
$$

where $\Omega$ is a bounded $C^{1,1}$ domain in $\mathbb{R}^{n}, \lambda \in \mathbb{R}, n \geq 1, c, h \in L^{p}(\Omega), M$ is a bounded matrix, and $F$ is a fully nonlinear uniformly elliptic operator of Isaacs type. As it was already seen (chapter 1), a particular case, for which all our multiplicity results are new as well, is when $F$ is a linear operator in nondivergence form (1.1).

In the recent years have appeared a series of papers which unveil the complex nature of the solution set for noncoercive equations $(\lambda c \supsetneqq 0)$, in the particular case when $F$ is the Laplacian. In 2013, Jeanjean and Sirakov [17] used a mountain pass argument related to Cerami sequences, when a classical exponential change of variables (as in lemma 5.11) reduces the equation to a semilinear one in linear and divergence form. They extended the multiplicity result in [12] under a smallness condition over $c$ and $\mu h$ (similar to the condition on quadratic $\mathrm{ABP}$ ) in the case $M=$ const.I.

Later Arcoya, de Coster, Jeanjean and Tanaka [13] (2015, see also [82]) developed a method based on degree theory which applies to general gradient terms. They stablished the existence of a continuum of solutions for the problem with $F=\Delta, M(x)=\mu(x) I$ for $0<\mu_{1} \leq \mu(x) \leq \mu_{2}$ and $h \supsetneqq 0$. Moreover, the authors proved the existence of a positive $\bar{\lambda}$ such that $\left(P_{\lambda}\right)$ there is no nonnegative solutions for $\lambda$ greater than $\bar{\lambda}$. Therefore it was realized that the sign of $h$ definitely matters for the problem; this because few years earlier (2007) Abdellaoui, Peral and Primo [83] exhibited one solution for the problem with $F=\Delta, M(x) \equiv I$, for a specific radial $c$ and $h \supsetneqq 0$, for all $\lambda>0$.

The work [13] was extended and completed by Jeanjean and Quorin [16] and by Jeanjean and de Coster [15]. In the latter the authors gave a description of the solution set in terms of the parameter $\lambda$; the sign of $h$ could be replaced by a more general condition over the sign of $u_{0}$, which is the solution of $\left(P_{0}\right)$. In fact, they obtained a continuum when $\left(P_{0}\right)$ has a solution and the existence 
of at least two solutions, for every $\lambda<\bar{\lambda}$, with $\bar{\lambda}$ being finite for $u_{0} \supsetneqq 0$ and infinite if $u_{0} \supsetneqq 0$; still for the laplacian and $M(x)=\mu(x) I$. A result with the $p$-Laplacian is found in [14].

Souplet [18] showed that the study of $\left(P_{\lambda}\right)$ is even more difficult if $M$ is allowed to vanish somewhere (note that in any case a hypothesis which prevents $M \equiv 0$ is necessary). In all these works the crucial a priori bounds for $u$ in the $L^{\infty}$-norm rely on the fact that the second order operator is the Laplacian, or a divergence form operator.

Hence, it appears to be a natural question if this same kind of multiplicity result can be extended to a more general setting, namely for a nonlinearity $F$ in the nondivergence form, $\left(P_{\lambda}\right)$ given in the $L^{p}$-viscosity sense. In the sequel we see that the answer is affirmative. In other words, it is our goal here to perform a similar study for general operators in nondivergence form, and extend the results from [12] to noncoercive equations. Since $c \supsetneqq 0$, this means to give some description of the solution set of $\left(P_{\lambda}\right)$ when the parameter $\lambda$ is positive, similar to [15].

\section{1}

\section{Main Results}

We start this section by introducing our hypotheses.

From now on $\Omega$ is a bounded domain in $\mathbb{R}^{n}$ with $C^{1,1}$ boundary. We assume that the matrix $M$ satisfies the nondegeneracy condition

$$
\mu_{1} I \leq M(x) \leq \mu_{2} I \text { a.e. in } \Omega
$$

for some $\mu_{1}, \mu_{2}>0$, and that $\left(P_{\lambda}\right)$ has the (SC) structure, recalling

$$
\begin{array}{r}
\mathcal{M}^{-}(X-Y)-b(x)|p-q|-d(x) \omega\left((r-s)^{+}\right) \\
\leq F(x, r, p, X)-F(x, s, q, Y) \\
\leq \mathcal{M}^{+}(X-Y)+b(x)|p-q|+d(x) \omega\left((s-r)^{+}\right) \text {a.e. } x \in \Omega \\
F(\cdot, 0,0,0) \equiv 0, \quad b, d, c, h \in L^{p}(\Omega), p>n, b, d \geq 0, \omega \text { a modulus. }
\end{array}
$$

Here, $\mathcal{M}^{ \pm}$are the Pucci's operators with constants $0<\lambda_{P} \leq \Lambda_{P}{ }^{1}$. We also consider $\mathcal{L}^{ \pm}[u]:=\mathcal{M}^{ \pm}\left(D^{2} u\right) \pm b(x)|D u|, b \in L_{+}^{p}(\Omega)$, for the corresponding ellipticity coefficients $\lambda_{P}, \Lambda_{P}$.

Notice that, for ease of notation, we included in (SC) the unboundedness condition over the coefficients. On the other hand, we make the convention

\footnotetext{
${ }^{1}$ We are denoting the ellipticity coefficients by $\lambda_{P}$ and $\Lambda_{P}$ instead of the usual $\lambda$ and $\Lambda$ in order to avoid any confusions with $\lambda$ in the problem $\left(P_{\lambda}\right)$.
} 
that all of them are bounded functions when using the following stronger assumption

$$
\begin{array}{r}
\mathcal{M}^{-}(X-Y)-b(x)|p-q| \leq F(x, r, p, X)-F(x, s, q, Y) \\
\leq \mathcal{M}^{+}(X-Y)+b(x)|p-q| \text { a.e. } x \in \Omega \\
F(\cdot, 0,0,0) \equiv 0, \quad b, c, h \in L^{\infty}(\Omega), b \geq 0,
\end{array}
$$

needed for most of our results. Observe that a very particular case of the last hypothesis appears when $F$ is the linear operator (1.1), but we can go much further, allowing $F$ to be an arbitrary supremum or infimum of such linear operators, i.e. a Hamilton-Jacobi-Bellman (HJB) operator, and even to be a sup-inf of linear operators (Isaacs operator).

We will also assume that for some $\theta>0, r_{0}>0$ and all $x_{0} \in \bar{\Omega}$

$$
\left(\frac{1}{r^{n}} \int_{B_{r}\left(x_{0}\right) \cap \Omega} \bar{\beta}_{F}\left(x, x_{0}\right)^{p}\right)^{\frac{1}{p}} \leq \theta, \quad \text { for all } r \leq r_{0}
$$

where $\bar{\beta}$ is defined in (3.5). As we already mentioned, this is satisfied, for instance, if $F(x, 0,0, X)$ is continuous in $x \in \bar{\Omega}$ (if $F$ is linear this means $a_{i j}(x)$ are continuous, as in chapter 1$)$. The conditions $(M)-(\mathrm{SC})-\left(H_{\beta}\right)$ guarantee that the $L^{p}$-viscosity solutions of $\left(P_{\lambda}\right)$ have global $C^{1, \alpha}$ regularity and estimates, by theorem 3.1 and remark 3.3.

Solutions of the Dirichlet problem $\left(P_{\lambda}\right)$ are understood in the $L^{p}$-viscosity sense and belong to $C(\bar{\Omega})$. Thus, we study and prove multiplicity of bounded solutions. We note that multiple unbounded solutions can easily be found for simple equations with natural growth. For instance, in [84] it was observed that $\Delta u=|D u|^{2}$ admits infinitely many weak solutions in $W_{0}^{1,2}\left(B_{1}\right)$, namely $u_{k}=\ln \left(\left(|x|^{2-n}-k\right)(1-k)^{-1}\right), 0 \leq k<1$, in the case $n>2$.

Remember that strong solutions of $\left(P_{\lambda}\right)$, for $\partial \Omega \in C^{1,1}$ are functions in $W^{2, p}(\Omega)$ which satisfy the equation almost everywhere. Strong solutions are $L^{p}$-viscosity solutions (see proposition 2.5). Conversely, if $F$ is for instance convex in the matrix $X$ and satisfies (SC) $)_{0}$ (such are the HJB operators), then $L^{p}$-viscosity solutions are strong from theorem 3.3; the convexity assumption can be removed in some cases but not in general, see [85]. For some of our results we will need to assume that $L^{p}$-viscosity solutions of $\left(P_{\lambda}\right)$ are strong see hypothesis $\left(H_{2}\right)$ below.

Since we want to study the way the nature of the solution set changes when we go from negative to positive zero order term, we will naturally assume that the problem with $\lambda=0$ has a solution. We also assume that the Dirichlet problem for $F$ is uniquely solvable, so that we can concentrate on the way 
the coefficients $c$ and $M$ influence the solvability. We now summarize these conditions on $F$. First, we assume that

$$
\text { the problem }\left(P_{0}\right) \text { has a strong solution } u_{0} \text {. }
$$

Further, setting $F[u]:=F\left(x, u, D u, D^{2} u\right)$, we assume that for each $f \in L^{p}(\Omega)$, there exists a unique $L^{p}$-viscosity solution of $\left\{\begin{array}{cl}-F[u]=f(x) & \text { in } \Omega \\ u=0 & \text { on } \partial \Omega\end{array} \quad\left(H_{1}\right)\right.$

Given $c, h$ for which we study $\left(P_{\lambda}\right)$, if $\left(\bar{P}_{\lambda}\right)$ denotes the problem $\left(P_{\lambda}\right)$ with $c$ and $h$ replaced by $\bar{c}$ and $\bar{h}$, we sometimes require that $L^{p}$-viscosity solutions $\bar{u}_{\lambda}$ of $\left(\bar{P}_{\lambda}\right)$ are such that

$$
\bar{u}_{\lambda} \in W^{2, p}(\Omega) \text {, for every } 0 \leq \bar{c} \leq c,|\bar{h}| \leq|h|+1+c
$$

We observe that, by Theorem 1(iii) of [12], the function $u_{0}$ is the unique $L^{p}$-viscosity solution of $\left(P_{0}\right)$. Theorem 1 (ii) of [12] shows that $\left(H_{0}\right)$ holds for instance if $M h$ has small $L^{p}$-norm (examples showing that in general this hypothesis cannot be removed are also found in that paper). Moreover, recall that $\left(H_{1}\right)$ and $\left(H_{2}\right)$ are both true if $F$ satisfies $(\mathrm{SC})_{0}$ and is convex or concave in $X$, by the results in [38] and chapter 3 .

We now state our main multiplicity results. The following theorem contains a crucial uniform estimate for solutions of $\left(P_{\lambda}\right)$, which is both important in itself and instrumental for the existence statements below.

Theorem 5.1 Let $\Omega \in C^{1,1}$ be a bounded domain. Suppose $(\mathrm{SC})_{0},\left(H_{0}\right)$ hold and let $\Lambda_{1}, \Lambda_{2}$ with $0<\Lambda_{1}<\Lambda_{2}$. Then every $L^{p}$-viscosity solution $u$ of $\left(P_{\lambda}\right)$ satisfies

$$
\left\|u^{-}\right\|_{L^{\infty}(\Omega)} \leq C, \text { for all } \lambda \in\left[0, \Lambda_{2}\right], \quad\left\|u^{+}\right\|_{L^{\infty}(\Omega)} \leq C, \text { for all } \lambda \in\left[\Lambda_{1}, \Lambda_{2}\right],
$$

where $C$ depends on $n, p, \mu_{1}, \Omega, \Lambda_{1}, \Lambda_{2},\|b\|_{L^{\infty}(\Omega)},\|c\|_{L^{\infty}(\Omega)},\|h\|_{L^{\infty}(\Omega)}$, $\left\|u_{0}\right\|_{L^{\infty}(\Omega)}, \lambda_{P}, \Lambda_{P}$, the $C^{1,1}$ character of the boundary, and the set where $c>0$.

It will be clear from the next theorems that the restrictions on $\lambda$ cannot be removed. As in the previous works, we use the following order in the space $E:=C^{1}(\bar{\Omega})$.

Definition 5.2 Let $u, v \in E$. We say that $u \ll v$ if for every $x \in \Omega$ we have $u(x)<v(x)$ and for $x_{0} \in \partial \Omega$ we have either $u\left(x_{0}\right)<v\left(x_{0}\right)$, or $u\left(x_{0}\right)=v\left(x_{0}\right)$ and $\partial_{\nu} u\left(x_{0}\right)<\partial_{\nu} v\left(x_{0}\right)$ (recall that $\vec{\nu}$ is the interior unit normal to $\partial \Omega$ ). 
The hypotheses $\Omega \in C^{1,1}$ bounded domain, $c \supsetneqq 0$ and $(M)$ are implied attached to the problem $\left(P_{\lambda}\right)$; so we will no longer mention it each time.

Theorem 5.3 Assume (SC), $\left(H_{\beta}\right),\left(H_{0}\right)$, and $\left(H_{1}\right)$.

1. Then, for $\lambda \leq 0$, the problem $\left(P_{\lambda}\right)$ has an $L^{p}$-viscosity solution $u_{\lambda}$ that converges to $u_{0}$ in $E$ as $\lambda \rightarrow 0^{-}$. Moreover, the set

$$
\Sigma=\left\{(\lambda, u) \in \mathbb{R} \times E ; u \text { solves }\left(P_{\lambda}\right)\right\}
$$

possesses an unbounded component $\mathcal{C}^{+} \subset[0,+\infty] \times E$ such that $\mathcal{C}^{+} \cap(\{0\} \times E)$ reduces to $\left\{u_{0}\right\}$. From now on we assume $(\mathrm{SC})_{0}$.

2. The component from 1. is such that

(i) either it bifurcates from infinity to the right of the axis $\lambda=0$ with the corresponding solutions having a positive part blowing up to infinity in $C(\bar{\Omega})$ as $\lambda \rightarrow 0^{+}$;

(ii) or its projection on the $\lambda$ axis is $[0,+\infty)$.

3. There exists $\bar{\lambda} \in(0,+\infty]$ such that, for every $\lambda \in(0, \bar{\lambda})$, the problem $\left(P_{\lambda}\right)$ has at least two $L^{p}$-viscosity solutions, $u_{\lambda, 1}$ and $u_{\lambda, 2}$, satisfying

$$
u_{\lambda, 1} \underset{\lambda \rightarrow 0^{+}}{\longrightarrow} u_{0} \text { in } E, \quad \max _{\bar{\Omega}} u_{\lambda, 2} \underset{\lambda \rightarrow 0^{+}}{\longrightarrow}+\infty
$$

and, if $\bar{\lambda}<+\infty$, the problem $\left(P_{\bar{\lambda}}\right)$ has at least one $L^{p}$-viscosity solution. The latter is unique if $F(x, r, p, X)$ is convex in $(r, p, X)$.

4. If in addition $\left(\mathrm{H}_{2}\right)$ holds, the solutions $u_{\lambda}$ for $\lambda \leq 0$ are unique among $L^{p}$-viscosity solutions; whereas the solutions from 3. for $\lambda>0$ are ordered, $u_{\lambda, 1} \ll u_{\lambda, 2}$.

This theorem proves the multiplicity conjectures from [12], [86]. We recall that theorem 5.3 is new even when $F$ is a linear operator in nondivergence form - in this case, it reduces to theorem 1.3.

The supplementary hypotheses for the uniqueness results in the above theorem are unavoidable - we recall that, in the universe of $L^{p}$-viscosity solutions, uniqueness is only available in the presence of a strong solution (see [12] and the references in that paper).

In the next two theorems, we show that it is possible to obtain a more precise description of the set $\Sigma$, provided we know the sign of $u_{0}$. Such results for the divergence case $F=\Delta$ were already proved in theorems 1.4 and 1.5 in [15]. Note that if $h$ has a sign, then $u_{0}$ has the same sign, by the maximum principle (see remark 6.32). 
Theorem 5.4 Suppose $(\mathrm{SC})_{0},\left(H_{\beta}\right),\left(H_{1}\right),\left(H_{2}\right)$ and $\left(H_{0}\right)$ with $u_{0} \leq 0$ and $c u_{0} \supsetneqq 0$. Then every nonpositive solution of $\left(P_{\lambda}\right)$ with $\lambda>0$ satisfies $u \ll u_{0}$. Furthermore, for every $\lambda>0$, the problem $\left(P_{\lambda}\right)$ has at least two nontrivial $L^{p}$-viscosity solutions $u_{\lambda, 1} \ll u_{\lambda, 2}$, such that $u_{\lambda_{2}, 1} \ll u_{\lambda_{1}, 1} \ll u_{0}$ if $0<\lambda_{1}<\lambda_{2}$, and

$$
u_{\lambda, 1} \underset{\lambda \rightarrow 0^{+}}{\longrightarrow} u_{0} \text { in } E, \quad \max _{\bar{\Omega}} u_{\lambda, 2} \underset{\lambda \rightarrow 0^{+}}{\longrightarrow}+\infty
$$

If $F(x, r, p, X)$ is convex in $(r, p, X)$ then $\max _{\bar{\Omega}} u_{\lambda, 2}>0$ for all $\lambda>0$.

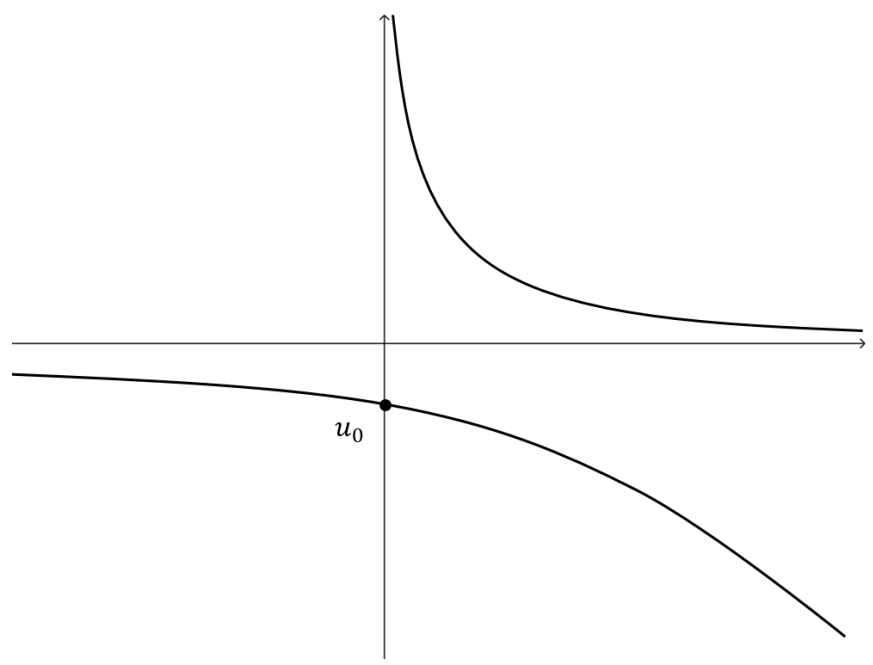

Figure 5.1: Illustration of theorem 5.4.

In this figure we put $\lambda$ on the horizontal axis. On the negative side of the vertical axis we have $u_{\lambda, 1}\left(x_{0}\right)$ for any fixed $x_{0} \in \Omega$ (or $\min _{\bar{\Omega}} u_{\lambda, 1}$ ), which is a negative quantity for $\lambda>0$; whereas on the positive side of the vertical axis we find $\left\|u_{\lambda, 2}\right\|_{L^{\infty}(\Omega)}\left(\right.$ or $\max _{\bar{\Omega}} u_{\lambda, 2}$ if $F$ is convex).

Theorem 5.5 Suppose $(\mathrm{SC})_{0},\left(H_{\beta}\right),\left(H_{1}\right),\left(H_{2}\right)$ and $\left(H_{0}\right)$ with $u_{0} \geq 0$ and $c u_{0} \supsetneqq 0$. Then every nonnegative solution of $\left(P_{\lambda}\right)$ with $\lambda>0$ satisfies $u \gg u_{0}$. Moreover, there exists $\bar{\lambda} \in(0,+\infty)$ such that

(i) for every $\lambda \in(0, \bar{\lambda})$, the problem $\left(P_{\lambda}\right)$ has at least two nontrivial $L^{p}$-viscosity solutions with $u_{\lambda, 1} \ll u_{\lambda, 2}$, where $u_{0} \ll u_{\lambda_{1}, 1} \ll u_{\lambda_{2}, 1}$ if $0<\lambda_{1}<\lambda_{2}$ and

$$
u_{\lambda, 1} \underset{\lambda \rightarrow 0^{+}}{\longrightarrow} u_{0} \text { in } E, \quad \max _{\bar{\Omega}} u_{\lambda, 2} \underset{\lambda \rightarrow 0^{+}}{\longrightarrow}+\infty
$$

(ii) the problem $\left(P_{\bar{\lambda}}\right)$ has at least one $L^{p}$-viscosity solution $u_{\bar{\lambda}}$; this solution is unique if $F$ is convex in $(r, p, X)$; 


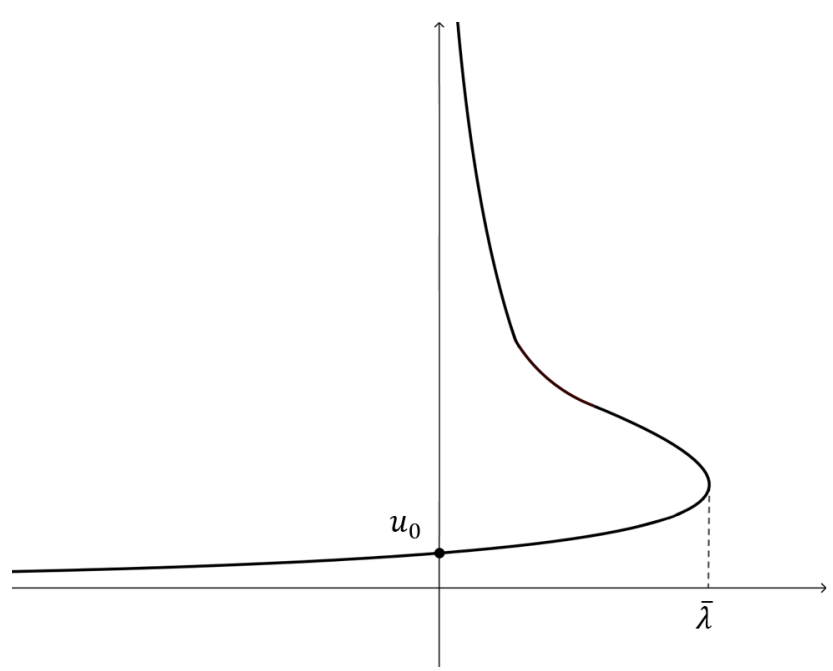

Figure 5.2: Illustration of theorem 5.5.

(iii) for $\lambda>\bar{\lambda}$, the problem $\left(P_{\lambda}\right)$ has no nonnegative solution.

Notice that, up to replacing $u$ for $-u$, we are also taking into account, indirectly, the case $-\mu_{2} I \leq M(x) \leq-\mu_{1} I$ for $\mu_{1}, \mu_{2}>0$. We only need to pay attention to the sign of $u_{0}$, which is reversed in this case.

\section{2}

\section{A Priori Bounds}

In this section we look at our family of problems $\left(P_{\lambda}\right)$ for $\lambda>0$, assuming $c \supsetneqq 0$ and that the matrix $M$ satisfies $(M)$. Here we consider $(\mathrm{SC})_{0}$ i.e. we suppose that all coefficients of the problem $\left(P_{\lambda}\right)$ are bounded and $d \equiv 0$. With the latter, the zero order term in $\left(P_{\lambda}\right)$ is explicit, so we can obtain a clear behavior of the solutions with respect to $\lambda$.

Theorem 5.6 Let $\Omega \in C^{1,1}$ be a bounded domain. Suppose $(\mathrm{SC})_{0}$, $\left(H_{0}\right)$ hold and let $\Lambda_{1}, \Lambda_{2}$ with $0<\Lambda_{1}<\Lambda_{2}$. Then every $L^{p}$-viscosity solution $u$ of $\left(P_{\lambda}\right)$ satisfies

$$
\|u\|_{\infty} \leq C, \text { for all } \lambda \in\left[\Lambda_{1}, \Lambda_{2}\right]
$$

where $C$ depends on $n, p, \mu_{1}, \Omega, \Lambda_{1}, \Lambda_{2},\|b\|_{L^{\infty}(\Omega)},\|c\|_{L^{\infty}(\Omega)},\|h\|_{L^{\infty}(\Omega)},\left\|u_{0}\right\|_{L^{\infty}(\Omega)}$, on the ellipticity coefficients $\lambda_{P}, \Lambda_{P}$, on the $C^{1,1}$ character of the boundary, and on the set where $c>0$.

The proof of theorem 5.6 uses and develops the ideas sketched in [86], adding some improvements in order to remove restrictions on the size of $c$. We start by proving that all supersolutions stay uniformly bounded from below, even when $\lambda$ is close to zero. 
Proposition 5.7 Let $\Omega \in C^{1,1}$ be a bounded domain. Suppose (SC) $)_{0}$ and let $\Lambda_{2}>0$. Then every $L^{p}$-viscosity supersolution $u$ of $\left(P_{\lambda}\right)$ satisfies

$$
\left\|u^{-}\right\|_{\infty} \leq C, \text { for all } \lambda \in\left[0, \Lambda_{2}\right]
$$

where $C$ depends only on $n, p, \mu_{1}, \Lambda_{2},\|b\|_{L^{\infty}(\Omega)},\|c\|_{L^{\infty}(\Omega)},\left\|h^{-}\right\|_{L^{\infty}(\Omega)}, \lambda_{P}, \Lambda_{P},|\Omega|$ and on the $C^{1,1}$ norm of $\partial \Omega$.

Proof. First observe that both $-u$ and 0 are $L^{p}$-viscosity subsolutions of

$$
\widetilde{F}\left(x, U, D U, D^{2} U\right) \leq \lambda c(x) U-\mu_{1}|D U|^{2}+h^{-}(x) \text { in } \Omega,
$$

where $\widetilde{F}(x, r, p, X)=-F(x,-r,-p,-X)$. Then, using $(\mathrm{SC})_{0}$, these functions are also $L^{p}$-viscosity subsolutions of

$$
\left\{\begin{aligned}
\mathcal{M}^{+}\left(D^{2} U\right)+b(x)|D U|-\mu_{1}|D U|^{2} & \geq-\lambda c(x) U-h^{-}(x) & & \text { in } \Omega \\
U & \leq 0 & & \text { on } \partial \Omega
\end{aligned}\right.
$$

and so is $U:=u^{-}=\max \{-u, 0\}$, as the maximum of subsolutions. Moreover, $U \geq 0$ in $\Omega$ and $U=0$ on $\partial \Omega$. We make the following exponential change

$$
w:=\frac{1-e^{-m U}}{m}, \quad \text { with } m=\frac{\mu_{1}}{\Lambda_{P}}
$$

where $\Lambda_{P}$ is the constant from the definition of Pucci's operators. From lemma 2.20, we know that $w$ is an $L^{p}$-viscosity solution of

$$
\left\{\begin{aligned}
-\mathcal{L}_{1}^{+}[w] & \leq h^{-}(x)+\frac{\lambda}{m} c(x)|\ln (1-m w)|(1-m w) & & \text { in } \quad \Omega \\
w & =0 & & \text { on } \partial \Omega
\end{aligned}\right.
$$

where $\mathcal{L}_{1}^{+}[w]:=\mathcal{L}^{+}[w]-m h^{-}(x) w$. Notice that the logarithm above is well defined, since

$$
0 \leq w=\frac{1}{m}\left\{1-e^{-m U}\right\}<\frac{1}{m} \quad \text { in } \Omega .
$$

Now set $w_{1}:=\frac{1}{m}\left(1-e^{-m u_{1}^{-}}\right)$, where $u_{1}$ is some fixed supersolution of $\left(P_{\lambda}\right), \lambda \geq 0$ (if there was not such supersolution, we would have nothing to prove). Then, by the above argument, $w_{1} \in[0,1 / m)$ is an $L^{p}$-viscosity solution of $\left(Q_{\lambda}\right)$. Define $\bar{w}:=\sup \mathcal{A}$, where

$$
\mathcal{A}:=\left\{w: w \text { is an } L^{p} \text {-viscosity solution of }\left(Q_{\lambda}\right) ; 0 \leq w<1 / m \text { in } \Omega\right\} .
$$

Then $\mathcal{A} \neq \emptyset$, since $w_{1} \in \mathcal{A}$, and $w_{1} \leq \bar{w} \leq 1 / m$ in $\Omega$. Also, as a supremum of subsolutions (locally bounded, since it belongs to the interval $[0,1 / m]$ ), $\bar{w}$ 
is an $L^{p}$-viscosity solution ${ }^{2}$ (possibly discontinuous) of the first inequality in $\left(Q_{\lambda}\right)$. Clearly, $\bar{w}=0$ on $\partial \Omega$.

Observe that $\mathcal{L}_{1}^{+}$is a coercive operator and the function

$$
f(x):=f_{\lambda}(x, \bar{w}(x))=h^{-}(x)+\frac{\lambda}{m} c(x)|\ln (1-m \bar{w})|(1-m \bar{w}) \in L_{+}^{p}(\Omega)
$$

with

$$
\left\|f^{+}\right\|_{L^{p}(\Omega)} \leq\left\|h^{-}\right\|_{L^{p}(\Omega)}+\frac{\Lambda_{2}}{m}\|c\|_{L^{p}(\Omega)} C_{0}
$$

since $A(\bar{w}):=|\ln (1-m \bar{w})|(1-m \bar{w}) \leq C_{0}$. Indeed, from $\lim _{t \rightarrow 0^{+}} t \ln t=0$ there exists a $\delta \in(0,1)$ such that $t|\ln t| \leq 1$, for all $0<t<\delta$ i.e. $A(\bar{w}) \leq 1$ when $x \in\left\{\bar{w}>\frac{1-\delta}{m}\right\}$. If $x \in\left\{\bar{w} \leq \frac{1-\delta}{m}\right\}$ then $A(\bar{w}) \leq|\ln \delta|$ (notice that $1-m \bar{w} \leq 1$ ) so take $C_{0}=\max \{1,|\ln \delta|\}$.

Therefore, by the proof of the boundary Lipschitz bound (see theorem 2.3 in [23], which does not require any continuity assumption on $\bar{w}$ ),

$$
\bar{w}(x) \leq C\left\|f^{+}\right\|_{L^{p}(\Omega)} \operatorname{dist}(x, \partial \Omega) \rightarrow 0 \quad \text { as } \quad x \rightarrow \partial \Omega
$$

and so $\bar{w} \not \equiv \frac{1}{m}$. Observe that the function $\bar{w}$ can be equal to $1 / m$ at some interior points.

If there was a sequence of supersolutions $u_{k}$ of $\left(P_{\lambda}\right)$ in $\Omega$ with unbounded negative parts, then there would exist a subsequence such that

$$
u_{k}^{-}\left(x_{k}\right)=\left\|u_{k}^{-}\right\|_{\infty} \underset{k \rightarrow \infty}{\longrightarrow}+\infty, \quad x_{k} \in \bar{\Omega}, \quad x_{k} \underset{k}{\rightarrow} x_{0} \in \bar{\Omega}
$$

with $x_{k} \in \Omega$ for large $k$, since $u_{k} \geq 0$ on $\partial \Omega$. Then the respective sequence

$$
w_{k}\left(x_{k}\right)=\frac{1}{m}\left\{1-e^{-m u_{k}^{-}\left(x_{k}\right)}\right\} \underset{k \rightarrow \infty}{\longrightarrow} \frac{1}{m}, w_{k} \in \mathcal{A}
$$

i.e. for every $\varepsilon>0$, there exists some $k_{0} \in \mathbb{N}$ such that

$$
\frac{1}{m} \geq \bar{w}\left(x_{k}\right) \geq w_{k}\left(x_{k}\right) \geq \frac{1}{m}-\varepsilon, \quad \text { for all } k \geq k_{0}
$$

thus there exists the $\left.\operatorname{limit}_{\lim _{k}} \bar{w}_{(} x_{k}\right)=\frac{1}{m}$ and also

$$
\bar{w}\left(x_{0}\right) \geq \varliminf_{x_{k} \rightarrow x_{0}} \bar{w}\left(x_{k}\right)=\lim _{k \rightarrow \infty} \bar{w}\left(x_{k}\right)=\frac{1}{m} .
$$

${ }^{2}$ If we need to use the fact that the supremum of $C$-viscosity subsolutions is still a $C$-viscosity subsolution, we apply theorem 4.2 in [33] for continuous equations. Indeed, in the problem $\left(Q_{\lambda}\right)$, we consider $\gamma, h_{0}$ and $c_{0}$ (instead of $b(x), h^{-}(x)$ and $c(x)$ ) such that $b(x) \leq \gamma, h^{-} \leq h_{0}, c(x) \leq c_{0}$, and the logarithm term on the right hand side being defined as 0 at points where $\bar{w}=1 / m$; we can also take $\mathcal{L}^{+}$instead of $\mathcal{L}_{1}^{+}$, since $m h^{-} w \geq 0$. 
Hence $x_{0} \in \Omega$, since $\bar{w}=0$ on $\partial \Omega$, and $\bar{w}\left(x_{0}\right)=\frac{1}{m}$.

Finally, define $z:=1-m \bar{w}$. Then $z$ is an $L^{p}$-viscosity supersolution of

$$
-\mathcal{L}_{1}^{-} z \geq-\lambda c(x)|\ln z| z \quad \text { in } \Omega, \quad z \nsupseteq 0 \text { in } \Omega, \quad z\left(x_{0}\right)=0,
$$

where $\mathcal{L}_{1}^{-}:=\mathcal{L}^{-}-m h^{-}$is a coercive operator. But this contradicts the following nonlinear version of the SMP.

Lemma 5.8 Set $\mathcal{L}_{1}^{-}[u]:=\mathcal{M}^{-}\left(D^{2} u\right)-\gamma|D u|-d u$, for a constant $d \geq 0$. Let $f \in C[0,+\infty)$ be defined by $f(s)=a s|\ln s|$ if $s>0, f(0)=0$, where $a \geq 0$. Then, the SMP holds for the operator $\mathcal{L}_{1}^{-}[\cdot]-f(\cdot)$, i.e. if $u$ is a C-viscosity solution (possibly discontinuous) of

$$
\left\{\begin{aligned}
\mathcal{L}_{1}^{-}[u] & \leq f(u) & \text { in } \Omega \\
u & \geq 0 & \text { in } \Omega
\end{aligned}\right.
$$

then either $u>0$ in $\Omega$ or $u \equiv 0$ in $\Omega$.

This lemma can be seen as a form of the Vazquez's strong maximum principle [87] for our operators, since one over the square root of the primitive of $z|\ln z|$ is not integrable at 0 . The proof of lemma 5.8 is given in section 5.2.1.

Note that we apply lemma 5.8 with $d=m\left\|h^{-}\right\|_{\infty}$ and $a=\Lambda_{2}\|c\|_{\infty}$.

Remark 5.9 Notice that, by lemma 2.7, given any pair of $L^{p}$-viscosity sub and supersolutions $\alpha, \beta$ of $\left(P_{0}\right)$, we have that $\alpha \leq u \leq \beta$ in $\Omega$, for any strong solution $u$ of $\left(P_{0}\right)$.

Before giving the proof of Theorem 5.6 we recall once more that the class of equations we study is invariant with respect to diffeomorphic changes of the spatial variable. In particular we can assume that the boundary of $\Omega$ is a hyperplane in a neighborhood of any given point of $\partial \Omega$. Indeed, straightening of the boundary leads to an equation of the same type, as showed in section 3.2.3, with bounds on the the coefficient norms depending on the $C^{1,1}$ norm of $\partial \Omega$.

Proof of Theorem 5.6. Fix $\Lambda_{1}, \Lambda_{2}$ with $0<\Lambda_{1}<\Lambda_{2}$. From proposition 5.7, there exists a constant $C_{1}>0$ such that

$$
u^{-} \leq C_{1}, \text { for every supersolution } u \text { of }\left(P_{\lambda}\right) \text {, for all } \lambda \in\left[0, \Lambda_{2}\right]
$$

Suppose then, in order to obtain a contradiction, that solutions are not bounded from above in $\left[\Lambda_{1}, \Lambda_{2}\right]$, by picking out a sequence $u_{k}$ of $L^{p}$-viscosity solutions of $\left(P_{\lambda}\right)$ such that

$$
u_{k}^{+}\left(x_{k}\right) \underset{k \rightarrow \infty}{\longrightarrow}+\infty, \quad x_{k} \in \bar{\Omega}, \quad x_{k} \underset{k}{\rightarrow} x_{0} \in \bar{\Omega}
$$


where $x_{k}$ is the point of maximum of $\left|u_{k}\right|$ in $\bar{\Omega}$, i.e. $\left\|u_{k}\right\|_{\infty}=\left|u\left(x_{k}\right)\right|$. Here, $\left\|u_{k}\right\|_{\infty}=u_{k}^{+}\left(x_{k}\right)+u_{k}^{-}\left(x_{k}\right)$ and $u_{k}^{-}\left(x_{k}\right) \in\left[0, C_{1}\right]$.

We claim that, up to changing the blow-up limit point $x_{0}$, we can suppose that there is a ball around $x_{0}$ in which $c$ is not identically zero.

To prove the claim, consider $G$, a maximal domain such that $c \equiv 0$ in $G$. Obviously there is no need of such argument if $|\{c=0\}|=0$ or even if $c \supsetneqq 0$ in a neighborhood of $x_{0}$. Suppose, hence, that $x_{0}$ is an interior point of $G$, and so $x_{k} \in G$ for large $k$ (considering a half ball in $G$ if $x_{0} \in \partial \Omega$, after a diffeomorphic change of independent variable which straightens the boundary). Notice that both $u_{k}$ and $u_{0}$ satisfy the same equation

$$
-F\left(x, u, D u, D^{2} u\right)=\langle M(x) D u, D u\rangle+h(x) \quad \text { in } G
$$

in the $L^{p}$-viscosity sense, for each $k \in \mathbb{N}$ (recall $c=0$ in $G$ ). Because of $(\mathrm{SC})_{0}$ we have $F(x, r, p, X)=F(x, r+a, p, X)$ for $a \in \mathbb{R}$, hence the functions $v_{k}:=u_{k}-\inf _{\partial G} u_{k}$ and $v_{0}:=u_{0}-\sup _{\Omega} u_{0}$ still satisfy equation (5.2). Also $v_{k} \leq 0 \leq v_{0}$ on $\partial G$, so $v_{k}$ and $v_{0}$ are respectively $L^{p}$-viscosity sub and supersolution of $\left(P_{0}\right)$, with $v_{0}$ strong. We apply lemma 2.7 (see remark 5.9) to obtain that $v_{k} \leq v_{0}$ in $G$ and, in particular, for large $k$,

$$
u_{k}^{+} \geq u_{k}^{+}\left(x_{k}\right)-2\left\|u_{0}\right\|_{L^{\infty}(\Omega)}-C_{1} \underset{k \rightarrow \infty}{\longrightarrow}+\infty \quad \text { on } \partial G
$$

This means that we have blow-up also at the boundary of $G$, in the sense that there exists a sequence $y_{k} \in \partial G$ with $u_{k}^{+}\left(y_{k}\right) \rightarrow+\infty$ and $y_{k} \rightarrow y_{0} \in \partial G$, as $k \rightarrow \infty$. Next, since $G$ is maximal, so $\partial G \subset \partial \Omega \cup \partial(\{c=0\})$, and using $u_{k}=0$ on $\partial \Omega$, we have $y_{k} \in \partial(\{c=0\})$. Therefore, we can take a ball $B_{r}\left(y_{0}\right)$ centered at $y_{0}$ (or a half ball if $y_{0} \in \partial \Omega$ ) which, by enlarging $r$ if necessary, becomes a neighborhood which meets the set $\{c>0\}$; in other words, such that $c \supsetneqq 0$ in $B_{r}\left(y_{0}\right)$. Hence, up to changing $x_{k}$ and $x_{0}$ by $y_{k}$ and $y_{0}$, we can suppose that $c \supsetneqq 0$ in $B_{r}\left(x_{0}\right)$, or in a half ball if $x_{0} \in \partial \Omega$, after straightening the boundary around $x_{0}$.

Suppose we are in the more difficult case of a half ball. For simplicity, and up to rescaling, say $c \supsetneqq 0$ in $B_{1}^{+}=B_{1}^{+}\left(x_{0}\right)$, with our equation being defined in $B_{2}^{+}\left(x_{0}\right) \subset \Omega$.

We make the convention of assuming that the constant $C$ may change from line to line and depends on $n, p, \lambda_{P}, \Lambda_{P}, \Lambda_{1}, \Lambda_{2}, \mu_{1},\|b\|_{L^{p}(\Omega)},\|h\|_{L^{p}(\Omega)}$, $\|c\|_{L^{p}(\Omega)}$ and $C_{1}$. The constant $C_{1}$ is fixed in (5.1) with its dependence described in the statement of proposition 5.7.

Notice that, from (5.1), for every $L^{p}$-viscosity solution $u$ of $\left(P_{\lambda}\right)$, the 
function $v:=u+C_{1}$ is a nonnegative $L^{p}$-viscosity solution of

$$
\begin{aligned}
\mathcal{M}^{-}\left(D^{2} v\right)-b(x)|D v| & \leq F\left(x, v-C_{1}, D v, D^{2} v\right) \\
& =-\lambda c(x) v-\langle M(x) D v, D v\rangle-h(x)+\lambda c(x) C_{1} \\
& \leq-\lambda c(x) v-\mu_{1}|D v|^{2}+\widetilde{h}(x)
\end{aligned}
$$

where $\widetilde{h}(x):=h^{-}(x)+\Lambda_{2} c(x) C_{1} \geq 0$, by (SC) $)_{0}$ Thus, by lemma 2.20 , the function

$$
v_{1}:=\frac{1}{m_{1}}\left\{e^{m_{1} v}-1\right\}, \text { where } m_{1}=\frac{\mu_{1}}{\Lambda_{P}}
$$

is a nonnegative $L^{p}$-viscosity supersolution of

$$
\mathcal{L}_{1}^{-}\left[v_{1}\right] \leq f_{1}(x) \text { in } B_{2}^{+}
$$

where $\mathcal{L}_{1}^{-}\left[v_{1}\right]:=\mathcal{M}^{-}\left(D^{2} v_{1}\right)-b(x)\left|D v_{1}\right|-m_{1} \widetilde{h}(x) v_{1}$ and

$$
f_{1}(x):=-\frac{\lambda}{m_{1}} c(x)\left(1+m_{1} v_{1}\right) \ln \left(1+m_{1} v_{1}\right)+\widetilde{h}(x) \in L^{p}(\Omega)
$$

since $v_{1} \in L^{\infty}(\Omega)$. Notice that, in the set $B_{2}^{+} \cap\left\{f_{1} \geq 0\right\}$, we have

$$
0 \leq \frac{\lambda}{m_{1}} c(x)\left(1+m_{1} v_{1}\right) \ln \left(1+m_{1} v_{1}\right) \leq \widetilde{h}
$$

and $f_{1}^{+}=\left|f_{1}\right| \leq \frac{\lambda}{m_{1}} c(x)\left(1+m_{1} v_{1}\right) \ln \left(1+m_{1} v_{1}\right)+\widetilde{h} \leq 2 \widetilde{h}$, so

$$
\left\|f_{1}^{+}\right\|_{L^{p}\left(B_{2}^{+}\right)} \leq 2\|\widetilde{h}\|_{L^{p}\left(B_{2}^{+}\right)} \leq 2\left\|h^{-}\right\|_{L^{p}(\Omega)}+2 \Lambda_{2}\|c\|_{L^{p}(\Omega)} C_{1} \leq C .
$$

Then, using proposition 2.15 (BQSMP, case $p=q>n$ ) applied to (5.4), we obtain positive constants $c_{0}, C_{0}$ and $\varepsilon \leq 1$, depending on $n, \lambda, \Lambda_{P}, p$ and $\|b\|_{L^{p}(\Omega)}$, such that

$$
\begin{aligned}
I & :=\inf _{B_{1}^{+}} \frac{v_{1}}{x_{n}} \geq c_{0}\left(\int_{B_{3 / 2}^{+}}\left(f_{1}^{-}\right)^{\varepsilon}\right)^{1 / \varepsilon}-C_{0}\left\|f_{1}^{+}\right\|_{L^{p}\left(B_{2}^{+}\right)} \\
& =c_{0}\left(\int_{B_{3 / 2}^{+}}\left\{\left(\frac{\lambda}{m_{1}} c(x)\left(1+m_{1} v_{1}\right) \ln \left(1+m_{1} v_{1}\right)-\widetilde{h}(x)\right)^{+}\right\}^{\varepsilon}\right)^{1 / \varepsilon}-C \\
& \geq c_{0} \inf _{B_{1}^{+}} \frac{v_{1}}{x_{n}}\left(\int _ { B _ { 1 } ^ { + } } \left(\left(\lambda c(x) \frac{1+m_{1} v_{1}}{m_{1} v_{1}} x_{n} \ln \left(1+m_{1} v_{1}\right)\right.\right.\right. \\
& \geq c_{0} I\left(\int_{B_{1}^{+}}\left\{\left(\lambda c(x) \frac{1+m_{1} v_{1}}{v_{1}} x_{n}\right)^{+}\right)^{\varepsilon}\right)^{\frac{1}{\varepsilon}}-C
\end{aligned}
$$


using (5.5) and that $v_{1}(x) \geq x_{n} I$ for all $x \in B_{1}^{+}$. Thus,

$$
I\left\{c_{0}\left(\int_{B_{1}^{+}} x_{n}^{\varepsilon}\left(\left(\lambda c(x) \ln \left(1+I m_{1} x_{n}\right)-m_{1} \tilde{h}(x)\right)^{+}\right)^{\varepsilon}\right)^{1 / \varepsilon}-1\right\} \leq \bar{C} .
$$

We claim that this is only possible if $I \leq C$, with a constant that does not depend on $v_{1}$ (and consequently on $u$ ), neither $\lambda \in\left[\Lambda_{1}, \Lambda_{2}\right]$. Indeed, if this was not the case, we would obtain a sequence of supersolutions $v_{1}^{k}$ of $\mathcal{L}_{1}^{-}\left[v_{1}^{k}\right] \leq f_{1}^{k}(x)$ in $B_{2}^{+}$such that $I_{k}:=\inf _{B_{1}^{+}} \frac{v_{1}^{k}}{x_{n}} \rightarrow+\infty$ when $k \rightarrow+\infty$ and (5.6) holding with $I$ replaced by $I_{k}$. So, up to a subsequence and renumbering, we can assume that $I_{k} \geq k^{2}$ and $\frac{\bar{C}}{k^{2}} \leq 1$ for all $k \geq k_{0}$, from where it follows

$$
\int_{B_{1}^{+}} x_{n}^{\varepsilon}\left(\left(\lambda c(x) \ln \left(1+I_{k} m_{1} x_{n}\right)-m_{1} \widetilde{h}(x)\right)^{+}\right)^{\varepsilon} \leq c_{0}^{-\varepsilon}\left(1+\frac{\bar{C}}{k^{2}}\right)^{\varepsilon} \leq C
$$

and finally, using $\lambda \geq \Lambda_{1}$,

$$
\int_{B_{1}^{+} \cap\left\{x_{n} \geq 1 / k\right\}} x_{n}^{\varepsilon}\left(\left(\Lambda_{1} c(x)-m_{1} \frac{\widetilde{h}(x)}{\ln \left(1+m_{1} k\right)}\right)^{+}\right)^{\varepsilon} \leq \frac{C}{\ln \left(1+m_{1} k\right)} .
$$

Passing to limits as $k \rightarrow+\infty$ we have $\int_{B_{1}^{+}}\left(x_{n} c(x)\right)^{\varepsilon} \mathrm{d} x=0$, since $\Lambda_{1}>0$, which contradicts $c(x) \supsetneqq 0$ in $B_{1}^{+}$. More precisely, for the limit in (5.7) we can use, for example, the dominated convergence theorem: for $\varepsilon=1$ this is obvious; for $0<\varepsilon<1$ we use Young's inequality to estimate

$$
\left(\left(\lambda c(x)-m_{1} \widetilde{h}(x) / \ln \left(1+m_{1} k\right)\right)^{+}\right)^{\varepsilon} \leq\left(\lambda c(x)-m_{1} \widetilde{h}(x) / \ln \left(1+m_{1} k\right)\right)^{+}+1
$$

ensuring the desired convergence. In this way we establish the claim

$$
\inf _{B_{1}^{+}} \frac{v_{1}}{x_{n}} \leq C
$$

Thus, by theorem 2.16 ( $B W H I$, again with $p=q>n$ ) applied to (5.4), we obtain that there exists other positive constants $\varepsilon, c_{0}, C_{0}$, depending on $n, \lambda_{P}, \Lambda_{P}, p$ and $\|b\|_{L^{p}(\Omega)}$, such that

$$
\left(\int_{B_{3 / 2}^{+}} v_{1}^{\varepsilon}\right)^{1 / \varepsilon} \leq c_{0}\left(\int_{B_{3 / 2}^{+}}\left(\frac{v_{1}}{x_{n}}\right)^{\varepsilon}\right)^{1 / \varepsilon} \leq \inf _{B_{1}^{+}} \frac{v_{1}}{x_{n}}+C_{0}\left\|f_{1}^{+}\right\|_{L^{p}\left(B_{2}^{+}\right)} \leq C .
$$

Now we go back to $u$ and define

$$
v_{2}:=\frac{1}{m_{2}}\left\{e^{m_{2} u}-1\right\}, \text { with } m_{2}=\frac{\mu_{2}}{\lambda_{P}}
$$


which by lemma 2.20 and $(\mathrm{SC})_{0}$ is an $L^{p}$-viscosity solution of

$$
\left\{\begin{array}{rlrl}
\mathcal{M}^{+}\left(D^{2} v_{2}\right)+b(x)\left|D v_{2}\right|+\nu(x) v_{2} & \geq-h^{+}(x) & \text { in } B_{2}^{+} \\
v_{2} & =0 & & \text { on } B_{2}^{0}
\end{array}\right.
$$

where $\nu(x):=\frac{\lambda}{m_{2} v_{2}} c(x)\left(1+m_{2} v_{2}\right) \ln \left(1+m_{2} v_{2}\right)+m_{2} h^{+}(x) \in L^{p}\left(B_{2}^{+}\right)$.

Notice that by the definitions (5.3) and (5.9),

$$
v_{2}=\frac{1}{m_{2}}\left\{\left(1+m_{1} v_{1}\right)^{\frac{m_{2}}{m_{1}}} e^{-m_{2} C_{1}}-1\right\}
$$

As in [15], observe that

$$
\lambda c(x)\left|\frac{\left(1+m_{2} v_{2}\right)}{m_{2} v_{2}} \ln \left(1+m_{2} v_{2}\right)\right| \leq C_{s} c(x)\left(1+\left|v_{2}\right|^{s}\right)
$$

for any $s>0$. Indeed, $v_{2} \geq R_{1}$, for $R_{1}:=\frac{1}{m_{2}}\left\{e^{-C_{1} m_{2}}-1\right\} \in\left(-1 / m_{2}, 0\right)$ by (5.1). Then, since $\lim _{t \rightarrow 0} \frac{\ln (1+t)}{t}=1$, there exists a $\delta \in\left(0,-R_{1}\right)$ such that the expression inside the modulus sign in (5.12) is less than $1+m_{2} \delta$, for every $x \in\left\{\left|v_{2}\right|<\delta\right\}$. Further, from $\lim _{t \rightarrow+\infty} \frac{1+t}{t}=1$ and $\lim _{t \rightarrow+\infty} \frac{\ln (1+t)}{t^{s}}=0$, there exists a $R_{2}>0$ such that this expression is less than $2 v_{2}{ }^{s}$, for all $x \in\left\{v_{2} \geq R_{2}\right\}$. Finally, the same term is bounded by a constant which depends on $R_{1}, R_{2}$ and $\delta$ if $v_{2} \in\left[R_{1},-\delta\right] \cup\left[\delta, R_{2}\right]$, and so (5.12) holds for any $s>0$.

Now, if we take $s=\varepsilon \frac{m_{1}}{m_{2}} \frac{p-n}{p(p+n)}$ and $p_{1}=\frac{p+n}{2} \in(n, p)$ then, by Holder's inequality, the right hand side in (5.12) belongs to $L^{p_{1}}\left(B_{2}^{+}\right)$and

$$
\begin{aligned}
\left\|c\left|v_{2}\right|^{s}\right\|_{L^{p_{1}\left(B_{2}^{+}\right)}} & \leq\|c\|_{L^{p}\left(B_{2}^{+}\right)}\left\|\left|v_{2}\right|^{s}\right\|_{L^{p_{2}\left(B_{2}^{+}\right)}}, \quad \text { with } \frac{1}{p_{1}}=\frac{1}{p}+\frac{1}{p_{2}} \\
& \leq\|c\|_{L^{p}\left(B_{2}^{+}\right)}\left(\int_{B_{2}^{+}}\left|v_{2}\right|^{\frac{m_{1}}{m_{2}}}\right)^{\frac{p-n}{p(p+n)}} \leq C
\end{aligned}
$$

and then

$$
\|\nu\|_{L^{p_{1}\left(B_{2}^{+}\right)}} \leq C_{n, p}\|c\|_{L^{p}(\Omega)}+\left\|c\left|v_{2}\right|^{s}\right\|_{L^{p_{1}\left(B_{2}^{+}\right)}}+m_{2}\left\|h^{+}\right\|_{L^{p}(\Omega)} \leq C .
$$

Next, the uniform bound on (5.14) allows us to use theorem 2.1 (BLMP, case $p=q>n$ ) applied to (5.10), for $v_{2}$ and $r=\varepsilon \frac{m_{1}}{m_{2}}$ as in (5.13), to obtain

$$
v_{2}^{+} \leq C\left\{\left(\int_{B_{3 / 2}^{+}}\left|v_{2}\right|^{r}\right)^{1 / r}+\left\|h^{+}\right\|_{L^{p}(\Omega)}\right\} \leq C \text { in } B_{1}^{+} .
$$

Hence, $u^{+}=\frac{1}{m_{2}} \ln \left(1+m_{2} v_{2}^{+}\right)$is uniformly bounded in $B_{1}^{+}$, for every

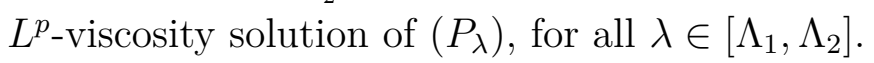




\subsection{1}

\section{Proof of Lemma 5.8}

We show that the result is valid for the most general notion of $C$-viscosity solutions (see [3], [33]), which can be discontinuous functions. We can suppose $u \in L S C(\Omega)$, just replacing $u$ by its lower semicontinuous envelope $u_{*}$, see chapter 2. Remember that, in this case, we say that $u$ is a $C$-viscosity supersolution provided $u_{*}$ is.

In this section, for simplicity, we denote $\mathcal{L}_{1}^{-}[u]=\mathcal{M}_{\lambda, \Lambda}^{-}\left(D^{2} u\right)-\gamma|D u|-d u$, with ellipticity coefficients $\lambda$ and $\Lambda$.

By contradiction, let $u$ be a nonnegative $C$-viscosity supersolution of

$$
\mathcal{L}_{1}^{-}[u] \leq f(u) \quad \text { in } \Omega
$$

with both $\Omega_{0}:=\{x \in \Omega ; u(x)=0\}$ and $\Omega^{+}:=\{x \in \Omega ; u(x)>0\}$ nonempty sets. Notice that $\Omega^{+}$is open, since $u \in L S C(\Omega)$. As in the usual proof of SMP (see, for instance, [39]), choose $\tilde{x} \in \Omega^{+} \operatorname{such}$ that $\operatorname{dist}\left(\tilde{x}, \Omega_{0}\right)<\operatorname{dist}(\tilde{x}, \partial \Omega)$ and consider the ball $B_{R}=B_{R}(\tilde{x}) \subset \Omega^{+}$such that $\partial B_{R}(\tilde{x}) \cap \partial \Omega_{0} \neq \emptyset$.

Observe that $f$ is a strictly increasing function on the interval $(0, \delta)$, for some $\delta<1$.

Fix a $x_{0} \in \partial B_{R}(\tilde{x}) \cap \partial \Omega_{0}$, so $u\left(x_{0}\right)=0$ and $u(x)>0$ in $B_{R}=B_{R}(\tilde{x})$. Note that, up to diminishing $R$, we can suppose also that $u<\delta$ in $B_{R}(\tilde{x})$. Indeed, since $u\left(x_{0}\right)=0$ there exists a ball $B_{r_{0}}\left(x_{0}\right)$ such that $u<\delta$ in this ball; so by taking $R_{1}<R$ with $B_{R_{1}}\left(\tilde{x}_{1}\right) \subset B_{r_{0}}\left(x_{0}\right)$ for a point $\tilde{x_{1}} \in \Omega^{+}$, now just replace $\tilde{x}, R$ by $\tilde{x}_{1}, R_{1}$.

Consider the annulus $E_{R}=B_{R} \backslash \bar{B}_{R / 2}$ centered in $\tilde{x}$ and set $\mu$ as the quantity $\mu=\min _{\partial B_{R / 2}} u \in(0, \delta)$. We need to find a good barrier in $E_{R}$ for our nonlinear problem. This is accomplished in the following claim.

Claim 5.10 There exists a nonnegative classical subsolution $v \in C^{2}\left(\bar{E}_{R}\right)$ of

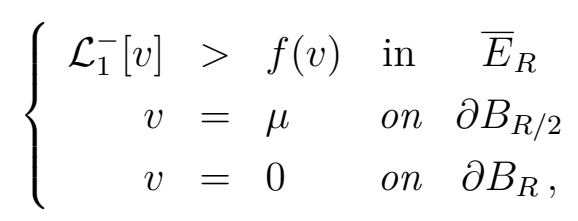

radially decreasing in $r=|x-\tilde{x}|$, convex and such that $\partial_{\nu} v>0$ on $\partial B_{R}$.

Proof. We start choosing a large $\alpha>1$ such that

$$
\frac{1}{R^{2}}\left\{\alpha[\lambda(\alpha+1)+(n-1) \Lambda-\gamma R]-d R^{2}\right\}>C_{0} \alpha,
$$

for $C_{0}:=a(|\ln \mu|+2|\ln R|+|\ln 2|+|\ln (R / 2)|)+m_{0}, m_{0}:=\max _{s \in[0,1]} f(s)>0$. 
Let $\varepsilon>0$ be such that $\varepsilon=\varepsilon(\alpha):=\mu\left\{(R / 2)^{-\alpha}-R^{-\alpha}\right\}^{-1}$, and set

$$
v(x):=\varepsilon\left\{|x-\tilde{x}|^{-\alpha}-R^{-\alpha}\right\} .
$$

Remark 5.11 (For instance, lemma 2.2 in [12].) Let $u \in C^{2}(B)$ be a radial function, $u(x)=\varphi\left(\left|x-x_{0}\right|\right)$, defined in a ball $B$ in $\mathbb{R}^{n}$ centered on $x_{0}$. Then

$$
\operatorname{spec}\left(D^{2} u(x)\right)=\left\{\frac{\varphi^{\prime}\left(\left|x-x_{0}\right|\right)}{\left|x-x_{0}\right|}, \ldots, \frac{\varphi^{\prime}\left(\left|x-x_{0}\right|\right)}{\left|x-x_{0}\right|}, \varphi^{\prime \prime}\left(\left|x-x_{0}\right|\right)\right\} .
$$

With this choice of $\varepsilon$, of course $v=\mu$ on $\partial B_{R / 2}$ and $v=0$ on $\partial B_{R}$. Notice that $v(x)=\varphi(r)$ with $r=|x-\tilde{x}|$, thus $\varphi^{\prime}(r)=-2 \alpha \varepsilon r^{-\alpha-1}<0$ and so

$$
\partial_{\nu} v(x)=D v(x) \cdot \vec{\nu}=-\varphi^{\prime}(R) \frac{x-\tilde{x}}{|x-\tilde{x}|} \cdot \frac{x-\tilde{x}}{|x-\tilde{x}|}=-\varphi^{\prime}(R)>0
$$

for every $x \in \partial B_{R}=\partial B_{R}(\tilde{x})$, where $\vec{\nu}=-\frac{x-\tilde{x}}{|x-\tilde{x}|}$ is the interior unit normal to the ball $B_{R}$. Further, $\varphi^{\prime \prime}(r)=\alpha(\alpha+1) \varepsilon r^{-\alpha-2}>0$, and by remark 5.11 we have, in $\bar{E}_{R}$,

$$
\begin{aligned}
\mathcal{L}_{1}^{-}[v]= & \mathcal{M}_{\lambda, \Lambda}^{-}\left(D^{2} v\right)-\gamma|D v|-d v(x) \\
= & \alpha \varepsilon|x-\tilde{x}|^{-\alpha-2}\{\lambda(\alpha+1)+(n-1) \Lambda\}-\gamma \alpha \varepsilon|x-\tilde{x}|^{-\alpha-1} \\
& \quad-d \varepsilon|x-\tilde{x}|^{-\alpha}+d \varepsilon R^{-\alpha} \\
\geq & \varepsilon|x-\tilde{x}|^{-\alpha} R^{-2}\left\{\alpha[\lambda(\alpha+1)+(n-1) \Lambda-\gamma R]-d R^{2}\right\} \\
> & \alpha \varepsilon C_{0}|x-\tilde{x}|^{-\alpha}
\end{aligned}
$$

by the choice of $\alpha$ in (5.15). Now we claim that

$$
f(v) \leq \alpha \varepsilon C_{0}|x-\tilde{x}|^{-\alpha} \text { in } \bar{E}_{R}
$$

and this will finish the proof of the claim 5.10. For $r=R$ this is obvious. Note that, for $r \neq R,(5.17)$ is equivalent to

$$
a\left(1-\frac{r^{\alpha}}{R^{\alpha}}\right)\left|\ln \varepsilon-\ln \left(r^{\alpha}\right)+\ln \left(1-\frac{r^{\alpha}}{R^{\alpha}}\right)\right| \leq \alpha C_{0}
$$

Set $y:=1-\left(\frac{r}{R}\right)^{\alpha} \in\left[0,1-\frac{1}{2^{\alpha}}\right]$ for $r \in\left[\frac{R}{2}, R\right]$. But now the left hand side of (5.18) is less or equal than

$$
\begin{aligned}
a|\ln \varepsilon| & +\alpha a|\ln r|+a y|\ln y| \\
& \leq a\left\{|\ln \mu|+\alpha|\ln R|+\left|\ln \left(2^{\alpha}-1\right)\right|\right\}+\alpha a\{|\ln R|+|\ln (R / 2)|\}+a y|\ln y| \\
& \leq \alpha C_{0}
\end{aligned}
$$


by using $\alpha>1$ and definitions of $\varepsilon$ and $m_{0}$.

By construction, $u \geq v$ on $\partial E_{R}=\partial B_{R} \cup \partial B_{R / 2}$.

Claim $5.12 u \geq v$ in $E_{R}$.

Proof. Suppose not, i.e. that the open set $\mathcal{O}:=E_{R} \cap\{u<v\}$ is not empty.

Notice that since $v$ is decreasing in $r$ and $v=\mu<\delta$ on $r=R / 2$, then both $u, v \in(0, \delta)$ in $E_{R}=B_{R} \backslash \bar{B}_{R / 2}$, and we can use the monotonicity of $f$ in the interval $(0, \delta)$ to obtain

$$
\left\{\begin{aligned}
\mathcal{L}_{1}^{-}[v]-\mathcal{L}_{1}^{-}[u] & >f(v)-f(u) \geq 0 & & \text { in } \mathcal{O} \\
v & \leq u & & \text { on } \partial \mathcal{O} \subset \partial E_{R} \cup\{v=u\}
\end{aligned}\right.
$$

in the $C$-viscosity sense, and so the comparison principle (for example proposition 3.3 in [33]), gives us that $v \leq u$ in $\mathcal{O}$, which contradicts the definition of the set $\mathcal{O}$.

To finish the proof of lemma 5.8, if $u \in C^{1}(\Omega)$, we can just use the fact that $u$ has a minimum at the interior point $x_{0}$, so by claim 5.12 we have $0=\partial_{\nu} u\left(x_{0}\right) \geq \partial_{\nu} v\left(x_{0}\right)>0$, a contradiction.

Assume $u$ is only in $\operatorname{LSC}(\Omega)$. Let $\rho<R / 2$. Observe that $v$ is a $C^{2}$ function in $B_{\rho}\left(x_{0}\right)$ with $v<0 \leq u$ in $B_{\rho}\left(x_{0}\right) \backslash E_{R}$ and, since $v \leq u$ in $E_{R}$ with $u\left(x_{0}\right)=v\left(x_{0}\right)=0$, then $v$ touches $u$ from below at the interior point $x_{0}$. Thus, the definition of $u$ being a $C$-viscosity supersolution on $\Omega$ yields

$$
\begin{aligned}
0 & <\mathcal{L}^{-}[v]\left(x_{0}\right)-d v\left(x_{0}\right)-f\left(v\left(x_{0}\right)\right)=\mathcal{L}^{-}[v]\left(x_{0}\right) \\
& =\mathcal{L}^{-}[v]\left(x_{0}\right)-d u\left(x_{0}\right)-f\left(u\left(x_{0}\right)\right) \leq 0,
\end{aligned}
$$

a contradiction. 


\section{6}

\section{The Degree Theory Approach}

This chapter is devoted to the proof of theorems 5.3, 5.4 and 5.5, split in sections 6.3, 6.4 and 6.5, respectively. However, the first section in the sequel is completely independent from the others sections and only requires the usual bounds necessary to the application of $C^{1, \alpha}$ regularity and estimates from chapter 3 .

\section{1}

\section{Existence results through fixed point theorems}

In this section we construct and study an auxiliary fixed point problem in order to obtain the existence statements in theorems 5.3-5.5.

Consider the problem $\left(P_{\lambda}\right)$ without $\lambda$ dependence, i.e.

$$
\left\{\begin{aligned}
-F\left(x, u, D u, D^{2} u\right) & =c(x) u+\langle M(x) D u, D u\rangle+h(x) & & \text { in } \Omega \\
u & =0 & & \text { on } \partial \Omega
\end{aligned}\right.
$$

under (SC). In this section all results hold for functions $b, c$ and $h$ in $L^{p}(\Omega)$. About the matrix $M$, we only need to assume that $M \in L^{\infty}(\Omega)$. We set $\mu=\|M\|_{L^{\infty}(\Omega)}$. As for $c$, no sign condition is needed in this section.

We define, under hypothesis $\left(H_{1}\right)$, the operator $\mathcal{T}: E \rightarrow E$ that takes a function $u \in E=C^{1}(\bar{\Omega})$ into $U=\mathcal{T} u$, the unique $L^{p}$-viscosity solution of the problem

$$
\left\{\begin{aligned}
-F\left(x, U, D U, D^{2} U\right) & =c(x) u+\langle M(x) D u, D u\rangle+h(x) & & \text { in } \Omega \\
U & =0 & & \text { on } \partial \Omega
\end{aligned}\right.
$$

Claim 6.1 The operator $\mathcal{T}$ is completely continuous.

Proof. Let $u_{k} \in E, u_{k} \rightarrow u$ in $E$. Then $\left\|u_{k}\right\|_{E} \leq C_{0}$, for all $k \in \mathbb{N}$. Set $f_{k}(x):=c(x) u_{k}+\left\langle M(x) D u_{k}, D u_{k}\right\rangle+h(x)$ so

$$
\left\|f_{k}\right\|_{L^{p}(\Omega)} \leq\|h\|_{L^{p}(\Omega)}+C_{0}\|c\|_{L^{p}(\Omega)}+\mu C_{0}^{2} \leq C .
$$

Next, $\mathrm{ABP}$ on the sequence $U_{k}=\mathcal{T} u_{k}$ produces $\left\|U_{k}\right\|_{L^{\infty}} \leq C\left\|f_{k}\right\|_{L^{p}(\Omega)} \leq C$. Therefore the $C^{1, \alpha}$ global estimate (theorem 3.1 and remark 3.3, see also 
remark 3.2) gives us $\left\|U_{k}\right\|_{C^{1, \alpha}(\bar{\Omega})} \leq C$. By the compact inclusion of $C^{1, \alpha}(\bar{\Omega})$ into $E$, there exists $U \in E$ and a subsequence such that $U_{k} \rightarrow U$ in $E$. This already shows that $\mathcal{T}$ takes bounded sets into precompact ones.

We need to see that $U=\mathcal{T} u$. This easily follows from stability (proposition 2.12), by defining, for each $\varphi \in W_{\text {loc }}^{2, p}(\Omega)$,

$$
g_{k}(x):=-F\left(x, U_{k}, D \varphi, D^{2} \varphi\right)-f_{k}(x), \quad g(x):=-F\left(x, U, D \varphi, D^{2} \varphi\right)-f(x)
$$

a.e. $x \in \Omega$, where $f$ is the same as $f_{k}$ with $u_{k}$ replaced by $u$. Indeed, by (SC),

$$
\begin{aligned}
& \left\|g_{k}-g\right\|_{L^{p}(\Omega)} \leq\|d\|_{L^{p}(\Omega)} \omega\left(\left\|U_{k}-U\right\|_{L^{\infty}(\Omega)}\right)+\|c\|_{L^{p}(\Omega)}\left\|u_{k}-u\right\|_{L^{\infty}(\Omega)} \\
& \quad+\mu\left(\left\|D u_{k}\right\|_{L^{\infty}(\Omega)}+\|D u\|_{L^{\infty}(\Omega)}\right)\left\|D u_{k}-D u\right\|_{L^{\infty}(\Omega)}|\Omega|^{1 / p} \\
& \quad \leq\|d\|_{L^{p}(\Omega)} \omega\left(\left\|U_{k}-U\right\|_{L^{\infty}(\Omega)}\right)+\left\{\|c\|_{L^{p}(\Omega)}+2 C_{0} \mu|\Omega|^{1 / p}\right\}\left\|u_{k}-u\right\|_{E},
\end{aligned}
$$

which converges to zero as $k \rightarrow+\infty$, since $\omega$ is increasing, continuous in 0 , with $\omega(0)=0$. Since the problem $\left(\mathcal{T}_{u}\right)$ has a unique solution, $U=\mathcal{T} u$. On the other hand, since this argument can be made for any subsequence of the original $\left(U_{k}\right)_{k \in \mathbb{N}}$, the whole sequence converges to $U=\mathcal{T} u$.

The next existence statement is a typical result about existence between sub and supersolutions, and it is a version of theorem 2.1 of [15] for fully nonlinear equations. We start with a definition.

Definition 6.2 An $L^{p}$-viscosity subsolution $\alpha \in E$ (supersolution $\beta$ ) of $(\mathrm{P})$ is said to be strict if every $L^{p}$-viscosity supersolution (subsolution) $u \in E$ of $(\mathrm{P})$ such that $\alpha \leq u(u \leq \beta)$ in $\Omega$, also satisfies $\alpha \ll u(u \ll \beta)$ in $\Omega$.

Set $\mathcal{B}_{r}=\mathcal{B}_{r}^{E}(0):=\left\{u \in E ;\|u\|_{E}<r\right\}$, for any $r>0, E=C^{1}(\bar{\Omega})$.

Theorem 6.3 Let $\Omega \subset \mathbb{R}^{n}$ be a bounded domain with $\partial \Omega \in C^{1,1}$. Suppose (SC), $\left(H_{\beta}\right)$ and $\left(H_{1}\right)$. Let $\alpha=\max _{1 \leq i \leq \kappa} \alpha_{i}, \beta=\min _{1 \leq j \leq \iota} \beta_{j}$, where $\alpha_{i}, \beta_{j} \in$ $W^{2, p}(\Omega)$ are strong sub and supersolutions of $(\mathrm{P})$ respectively, with $\alpha \leq \beta$ in $\Omega$. Then $(\mathrm{P})$ has an $L^{p}$-viscosity solution satisfying $\alpha \leq u \leq \beta$ in $\Omega$. Furthermore,

(i) If $\alpha$ and $\beta$ are strict in the sense of definition 6.2, then for large $R>0$ we have

$$
\operatorname{deg}(I-\mathcal{T}, \mathcal{S}, 0)=1
$$

where $\mathcal{S}=\mathcal{O} \cap \mathcal{B}_{R}$, for $\mathcal{O}=\mathcal{O}_{\alpha, \beta}:=\left\{u \in C_{0}^{1}(\bar{\Omega}) ; \alpha \ll u \ll \beta\right.$ in $\left.\Omega\right\}$.

(ii) If $\left(\mathrm{H}_{2}\right)$ holds, there exists a minimal solution $\underline{u}$ and a maximal solution $\bar{u}$ of $(\mathrm{P})$ in the sense that every (strong) solution $u$ of $(\mathrm{P})$ in the order interval $[\alpha, \beta]$ (i.e. such that $\alpha(x) \leq u(x) \leq \beta(x)$ for all $x \in \Omega$ ) satisfies

$$
\alpha \leq \underline{u} \leq u \leq \bar{u} \leq \beta \quad \text { in } \quad \Omega
$$


Notice that, under the assumptions of theorem 6.3 and by the global $C^{1, \alpha}$ estimate (theorem 3.1 and remark 3.3), every solution $u$ of $(\mathrm{P})$ satisfying $\alpha \leq u \leq \beta$ in $\Omega$ is such that

$$
\|u\|_{C^{1, \alpha}(\bar{\Omega})} \leq C
$$

where $C$ depends on $r_{0}, n, p, \mu,\|b\|_{L^{p}(\Omega)},\|c\|_{L^{p}(\Omega)},\|h\|_{L^{p}(\Omega)}, \omega(1)\|d\|_{L^{p}(\Omega)}$, $\operatorname{diam}(\Omega), \lambda_{P}, \Lambda_{P}$, on the $C^{1,1}$ character of the boundary and, of course, on the $L^{\infty}$ uniform bounds on $u$ given by $\|\alpha\|_{\infty}$ and $\|\beta\|_{\infty}$.

Proof. Consider any $R \geq \max \left\{C,\|\alpha\|_{E},\|\beta\|_{E}\right\}+1$, with $C$ from (6.1).

Part 1. Existence of a solution in the order interval $[\alpha, \beta]$.

First of all, we construct a modified problem, similar to but a little bit simpler than the one given in [15]. In order to avoid technicalities, consider $\kappa=\iota=1$; later we indicate the corresponding changes. We set

$$
\begin{aligned}
& f(x, r, p):=h(x)+c(x) r+\langle M(x) p, p\rangle \\
& \bar{f}(x, r, p):=h(x)+c(x) r+\langle\bar{M}(x) p, p\rangle
\end{aligned}
$$

for

$$
\bar{M}(x)=\bar{M}(x, p):= \begin{cases}M(x), & \text { if }|p|<R \\ M(x) \frac{R^{2}}{|p|^{2}}, & \text { if }|p| \geq R\end{cases}
$$

and also

$$
\widetilde{f}(x, r, p):= \begin{cases}\bar{f}(x, \alpha(x), D \alpha(x)), & \text { if } r<\alpha(x) \\ \bar{f}(x, r, p), & \text { if } \quad \alpha \leq r \leq \beta(x) \\ \bar{f}(x, \beta(x), D \beta(x)), & \text { if } r>\beta(x) .\end{cases}
$$

Consider the problems

$$
\begin{aligned}
-F[u]=f(x, u, D u) & \text { in } \Omega, \\
-F[u]=\bar{f}(x, u, D u) & \text { in } \Omega, \\
-F[u]=\widetilde{f}(x, u, D u) & \text { in } \Omega, \\
& \text { and } u=0 \quad \text { on } \partial \Omega .
\end{aligned}
$$

Notice that, by the construction of $\bar{f}, \tilde{f}$ and $|D \alpha|,|D \beta|<R$, we have

$$
\widetilde{f}(x, \alpha(x), D \alpha(x))=\bar{f}(x, \alpha(x), D \alpha(x))=f(x, \alpha(x), D \alpha(x)) \text { a.e. in } \Omega
$$


and the same for $\beta$. So, $\alpha, \beta$ are also a pair of strong sub and supersolutions for $(\overline{\mathrm{P}})$ and $(\widetilde{\mathrm{P}})$. Observe also that $\|\bar{M}\|_{L^{\infty}(\Omega)} \leq \mu$.

Claim 6.4 The operator $F(x, r, p, X)+\langle\bar{M}(x) p, p\rangle$ still satisfies $(S C)^{\mu}$.

Proof. We need to estimate the difference $A_{p, q}:=\langle\bar{M}(x, p) p, p\rangle-\langle\bar{M}(x, q) q, q\rangle$. First, if both $|p|,|q|<R$, then $\bar{M}(x, p)=\bar{M}(x, q)=M(x)$ and we have nothing to prove (see remark 2.2). In the second place, if both $|p|,|q| \geq R$ then

$$
A_{p, q}=\frac{R^{2}}{|p|^{2}}\langle M(x) p, p\rangle-\frac{R^{2}}{|q|^{2}}\langle M(x) q, q\rangle \leq\langle M(x) p, p\rangle-\frac{R^{2}}{|q|^{2}}\langle M(x) q, q\rangle .
$$

So, reversing the roles of $p, q$, it is enough to consider $|p|<R$ and $|q| \geq R$. In this case, the modulus of the last expression above is equal to

$$
\begin{gathered}
\frac{1}{\left|q^{2}\right|}|\langle M(x)|q| p,|q| p\rangle-\langle M(x) R q, R q\rangle| \leq \mu \frac{|| q|p-R q|}{|q|} \frac{|q||p|+R|q|}{|q|} \\
\leq \mu|p-R q /| q||(|p|+R) \leq \mu|p-q|(|p|+|q|)
\end{gathered}
$$

since the distance between $p$ and $q$ is greater ${ }^{1}$ or equal than the distance between $p$ and the projection of $q$ on the ball $B_{R}$.

By claim 6.4, (6.1) and the definition of $R$, every solution $u$ of $(\overline{\mathrm{P}})$ with $\alpha \leq u \leq \beta$ in $\Omega$ is $C^{1, \alpha}$ up to the boundary and satisfies

$$
\|u\|_{E}<R
$$

so $\bar{M}(x)=\bar{M}(x, D u)=M(x)$, and $u$ is a solution of the original problem (P).

Claim 6.5 Every $L^{p}$-viscosity solution $u$ of $(\widetilde{\mathrm{P}})$ satisfies $\alpha \leq u \leq \beta$ in $\Omega$, hence is a solution of $(\overline{\mathrm{P}})$, and, by the above, a solution of $(\mathrm{P})$.

Proof. Let $u$ be an $L^{p}$-viscosity solution of $(\widetilde{\mathrm{P}})$. As in the proof of lemma 2.7, assume $v:=u-\alpha$ is such that $\min _{\bar{\Omega}} v=v\left(x_{0}\right)<0$ in order to produce a contradiction. Since $v \geq 0$ on $\partial \Omega$, it follows that $x_{0} \in \Omega$. Consider, then, the set $\widetilde{\Omega}:=\{v<0\} \neq \emptyset$.

We claim that $v$ is an $L^{p}$-viscosity supersolution of

$$
\mathcal{M}^{-}\left(D^{2} v\right)-b(x)|D v| \leq 0 \text { in } \widetilde{\Omega}
$$

Indeed, let $\varphi \in W_{\text {loc }}^{2, p}(\widetilde{\Omega})$ and $\widehat{x} \in \widetilde{\Omega}$ such that $v-\varphi$ has a local maximum at $\widehat{x}$ in $\widetilde{\Omega}$. Then, $\alpha+\varphi \in W_{\text {loc }}^{2, p}(\widetilde{\Omega})$ and $u-(\alpha+\varphi)$ has also a local maximum at

${ }^{1}$ Observe that $|p-q|$ is the length of the opposite side to the obtuse angle. 
$\widehat{x}$. By definition of $u$ as an $L^{p}$-viscosity supersolution of $(\widetilde{\mathrm{P}})$, for every $\varepsilon>0$, there exists an $r>0$ such that for a.e. in $B_{r}(\widehat{x}) \cap \widetilde{\Omega}$,

$$
-F\left(x, u, D\left(\alpha+\varphi, D^{2}(\alpha+\varphi)\right)\right)-\bar{f}(x, \alpha, D \alpha) \geq-\varepsilon,
$$

since $u<\alpha$ in $\widetilde{\Omega}$. Further, from $(6.2),-F\left(x, \alpha, D \alpha, D^{2} \alpha\right)-\bar{f}(x, u, D \alpha) \leq 0$ a.e. in $\widetilde{\Omega}$. Subtracting this from (6.5) and using (SC), we obtain $\mathcal{L}^{-}[u] \leq \varepsilon$ a.e. in $B_{r}(\widehat{x}) \cap \widetilde{\Omega}$, since $d(x) \omega\left(v^{+}\right) \equiv 0$ in $\widetilde{\Omega}$. Hence, (6.4) is proved.

Now, (6.4) and ABP imply that $v \geq 0$ in $\widetilde{\Omega}$, contradicting the definition of $\widetilde{\Omega}$. So, $\min _{\bar{\Omega}} v \geq 0$ i.e. $u \geq \alpha$ in $\Omega$. Analogously, $u \leq \beta$ in $\Omega$.

Next, we move to build a solution for the operator $\widetilde{\mathcal{T}}: E \rightarrow E$ that takes a function $u \in E$ into $U=\widetilde{\mathcal{T}}_{u}$, the unique $L^{p}$-viscosity solution of the problem

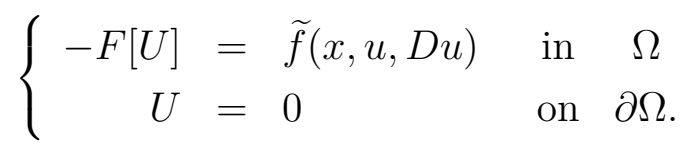

Solutions of $(\widetilde{\mathrm{P}})$ are fixed points of $\widetilde{\mathcal{T}}$, and belong to the order interval $[\alpha, \beta]$, by claim 6.5 . Moreover,

$$
\|\widetilde{\mathcal{T}} u\|_{E}<R_{0}, \quad \text { for all } u \in E
$$

for an appropriate $R_{0}>R$. In fact, by observing that

$$
|\langle\bar{M}(x) p, p\rangle|=\left\{\begin{array}{ll}
|\langle M(x) p, p\rangle|, & \text { if }|p|<R \\
\frac{R^{2}}{|p|^{2}}|\langle M(x) p, p\rangle|, & \text { if }|p| \geq R
\end{array} \leq \mu R^{2}, \text { for all } p \in \mathbb{R}^{n}\right.
$$

then $|\widetilde{f}(x, r, p)| \leq|h(x)|+\mu R^{2}+|c(x)| \max \left\{\|\alpha\|_{\infty},\|\beta\|_{\infty}\right\}$. In addition, for the function $\gamma(x):=\tilde{f}(x, u(x), D u(x))$,

$$
\|\gamma\|_{L^{p}(\Omega)} \leq\|h\|_{L^{p}(\Omega)}+\mu R^{2}+\|c\|_{L^{p}(\Omega)} \max \left\{\|\alpha\|_{\infty},\|\beta\|_{\infty}\right\}
$$

for every $u \in E$. Thus, $C^{1, \alpha}$ estimates (theorem 3.1 and remark 3.3, see also remark 3.2) together with $\mathrm{ABP}$, applied to the problem $\left(\widetilde{\mathcal{T}}_{u}\right)$, give us that $U=\widetilde{\mathcal{T}} u \in C^{1, \alpha}(\bar{\Omega})$ and $\|U\|_{C^{1, \alpha}(\bar{\Omega})} \leq C<R_{0}$. Thus, (6.6) follows.

Notice that $\widetilde{\mathcal{T}}$ takes bounded sets in $E$ into precompact ones, by the above and the compact inclusion $C^{1, \alpha} \subset E$. Also, if $u_{k} \rightarrow u$ in $E$, then $U_{k}=\widetilde{\mathcal{T}} u_{k} \rightarrow U$ in $E$ up to a subsequence; thus we can conclude that $\widetilde{\mathcal{T}}$ is completely continuous if we show that $U=\widetilde{\mathcal{T}} u$. To prove the latter, similarly to the argument with 
$\mathcal{T}$ we set, for $\varphi \in W_{\text {loc }}^{2, p}(\Omega)$,

$$
\begin{aligned}
g_{k}(x) & :=-F\left(x, U_{k}, D \varphi, D^{2} \varphi\right)-c(x) \widetilde{u}_{k}-\left\langle\bar{M}\left(x, D u_{k}\right) D u_{k}, D u_{k}\right\rangle-h(x) \\
g(x) & :=-F\left(x, U, D \varphi, D^{2} \varphi\right)-c(x) \widetilde{u}-\langle\bar{M}(x, D u) D u, D u\rangle-h(x),
\end{aligned}
$$

where, for each fixed function $v$, the function $\widetilde{v}$ is the following truncation

$$
\widetilde{v}(x):=\left\{\begin{array}{lll}
\alpha(x) & \text { if } \quad v(x)<\alpha(x) \\
v(x) & \text { if } \quad \alpha(x) \leq v(x) \leq \beta(x) \\
\beta(x) & \text { if } \quad v(x)>\beta(x)
\end{array}\right.
$$

Observe that

$$
\widetilde{u}_{k}(x)-\widetilde{u}(x)=\left\{\begin{array}{cll}
u_{k}(x)-u(x) & \text { if } x \in\left\{\alpha \leq u_{k} \leq \beta\right\} \cap\{\alpha \leq u \leq \beta\} \\
u_{k}(x)-\alpha(x) & \text { if } x \in\left\{\alpha \leq u_{k} \leq \beta\right\} \cap\{u<\alpha\} \\
u_{k}(x)-\beta(x) & \text { if } x \in\left\{\alpha \leq u_{k} \leq \beta\right\} \cap\{u>\beta\} \\
\alpha(x)-u(x) & \text { if } x \in\left\{u_{k}<\alpha\right\} \cap\{\alpha \leq u \leq \beta\} \\
\beta(x)-u(x) & \text { if } x \in\left\{u_{k}>\beta\right\} \cap\{\alpha \leq u \leq \beta\} \\
\beta(x)-\alpha(x) & \text { if } x \in\left\{u_{k}>\beta\right\} \cap\{u<\alpha\} \\
\alpha(x)-\beta(x) & \text { if } x \in\left\{u_{k}<\alpha\right\} \cap\{u>\beta\} \\
0 & \text { otherwise }
\end{array}\right.
$$

thus $\left|\widetilde{u}_{k}-\widetilde{u}\right|$ is given by

$$
\left\{\begin{array}{cll}
\left|u_{k}-u\right| & \text { in } \quad\left\{\alpha \leq u_{k} \leq \beta\right\} \cap\{\alpha \leq u \leq \beta\} \\
u_{k}-\alpha<u_{k}-u & \text { in } \quad\left\{\alpha \leq u_{k} \leq \beta\right\} \cap\{u<\alpha\} \\
\beta-u_{k}<u-u_{k} & \text { in } \quad\left\{\alpha \leq u_{k} \leq \beta\right\} \cap\{u>\beta\} \\
u-\alpha<u-u_{k} & \text { in } \quad\left\{u_{k}<\alpha\right\} \cap\{\alpha \leq u \leq \beta\} \\
\beta-u<u_{k}-u & \text { in } \quad\left\{u_{k}>\beta\right\} \cap\{\alpha \leq u \leq \beta\} \\
\beta-\alpha<\left|u_{k}-u\right| & \text { in } \quad\left(\left\{u_{k}>\beta\right\} \cap\{u<\alpha\}\right) \cup\left(\left\{u_{k}<\alpha\right\} \cap\{u>\beta\}\right) \\
0 & \text { otherwise }
\end{array}\right.
$$

and then $\left\|\widetilde{u}_{k}-\widetilde{u}\right\|_{L^{\infty}(\Omega)} \leq\left\|u_{k}-u\right\|_{L^{\infty}(\Omega)} \rightarrow 0$ as $k \rightarrow \infty$. By the estimates for $\mathcal{T}$ and using that the function $p \mapsto\langle\bar{M}(x, p) p, p\rangle$ is continuous in $p$ for a.e. $x \in \Omega$, we get $\left\|g_{k}-g\right\|_{L^{p}(\Omega)} \rightarrow 0$ as $k \rightarrow \infty$ and so $U=\tilde{\mathcal{T}} u$.

By complete continuity of $\mathcal{T}$ and (6.6), the degree $\operatorname{deg}\left(I-\tilde{\mathcal{T}}, \mathcal{B}_{R_{0}}, 0\right)$ is well defined and is equal to one. Indeed, set $H_{t}(u):=t \widetilde{\mathcal{T}} u$ for all $t \in[0,1]$ and notice that $\left(I-H_{t}\right) u=0 \Leftrightarrow u=t \widetilde{\mathcal{T}} u \in \mathcal{B}_{R_{0}}$. Then $I-H_{t}$ does not vanish 
on $\partial \mathcal{B}_{R_{0}}$ and

$$
\begin{aligned}
\operatorname{deg}\left(I-\widetilde{\mathcal{T}}, \mathcal{B}_{R_{0}}, 0\right) & =\operatorname{deg}\left(I-H_{1}, \mathcal{B}_{R_{0}}, 0\right) \\
& =\operatorname{deg}\left(I-H_{0}, \mathcal{B}_{R_{0}}, 0\right)=\operatorname{deg}\left(I, \mathcal{B}_{R_{0}}, 0\right)=1
\end{aligned}
$$

Therefore, $\tilde{\mathcal{T}}$ has a fixed point $u \in E$, which is a solution of $(\widetilde{\mathrm{P}})$. By claim 6.5, the first existence statement in theorem 6.3 is proved in the case $\kappa=\iota=1$.

If $\alpha$ and $\beta$ are in the general case as the maximum and minimum of strong sub and supersolutions, respectively, we define $\tilde{f}$ as

$$
\tilde{f}(x, r, p):= \begin{cases}\max _{1 \leq i \leq \kappa} \bar{f}\left(x, \alpha_{i}(x), D \alpha_{i}(x)\right), & \text { if } r<\alpha(x) \\ \bar{f}(x, r, p), & \text { if } \quad \alpha \leq r \leq \beta(x) \\ \min _{1 \leq j \leq \iota} \bar{f}\left(x, \beta_{j}(x), D \beta_{j}(x)\right), & \text { if } r>\beta(x)\end{cases}
$$

and consider $R>\max \left\{\left\|\alpha_{i}\right\|_{E},\left\|\beta_{j}\right\|_{E} ; 1 \leq i \leq \kappa, 1 \leq j \leq \iota\right\}$. In claim 6.5, choose $i \in\{1, \ldots, \kappa\}$ such that $\min _{\bar{\Omega}}(u-\alpha)=\left(u-\alpha_{i}\right)\left(x_{0}\right)$, define $v=u-\alpha_{i}$ and replace $\alpha$ by $\alpha_{i} \in W^{2, p}(\Omega)$ until the end of the proof, observing that $x \in \widetilde{\Omega}=\{v<0\}$ implies $u(x)<\alpha(x)$. The rest of the proof is exactly the same.

Denote by $\mathcal{H}$ the set of fixed points of $\mathcal{T}$ belonging to the order interval $[\alpha, \beta]$. In claim 6.5 we saw that this set contains the set of fixed points of $\tilde{\mathcal{T}}$. The converse is also true, since any solution of $(\mathrm{P})$ in the order interval $[\alpha, \beta]$ satisfies $\bar{f}(x, u, D u)=f(x, u, D u)$ and $(6.3)$, hence is a solution of $(\widetilde{\mathrm{P}})$, i.e.

$$
\mathcal{H}=\{u \in E ; u=\mathcal{T} u, \alpha \leq u \leq \beta \text { in } \Omega\}=\{u \in E ; u=\widetilde{\mathcal{T}} u\}
$$

Notice that we just proved that this set is nonempty.

Part 2. Degree computation in $\mathcal{S}$ under strictness of $\alpha, \beta$-proof of $(i)$

Suppose $\alpha, \beta$ are strict, and consider the set $\mathcal{S}$ as in the statement of theorem 6.3. Since there exists a solution $u \in C_{0}^{1}(\bar{\Omega})$ of $(\mathrm{P})$ with $\alpha \leq u \leq \beta$ in $\Omega$, by definition 6.2 we have $\alpha \ll u \ll \beta$ in $\Omega$ and so $\mathcal{S}$ is a nonempty open set in $C_{0}^{1}(\bar{\Omega})$. Further, from part 1 , we see that all fixed points of $\widetilde{\mathcal{T}}$ are in $\mathcal{S} \subset \mathcal{B}_{R} \subset \mathcal{B}_{R_{0}}$, the degree over $\mathcal{B}_{R_{0}}$ is equal to 1 and solutions of $(\mathrm{P})$ and $(\widetilde{\mathrm{P}})$ in $\mathcal{S}$ coincide, leading to

$$
\operatorname{deg}(I-\mathcal{T}, \mathcal{S}, 0)=\operatorname{deg}(I-\tilde{\mathcal{T}}, \mathcal{S}, 0)=\operatorname{deg}\left(I-\widetilde{\mathcal{T}}, \mathcal{B}_{R_{0}}, 0\right)=1
$$


Part 3. Existence of extremal solutions under $\left(H_{2}\right)$ - proof of (ii).

From the proof of part 1 , we know that for any sequence $\left(u_{k}\right)_{k \in \mathbb{N}}$ in $E$ with $u_{k} \rightarrow u$ in $E$ there exists $U \in E$ such that $\widetilde{\mathcal{T}} u_{k} \rightarrow U=\widetilde{\mathcal{T}} u$ in $E$. Then, if additionally $u_{k}=\widetilde{\mathcal{T}} u_{k}$, we obtain that $u=\widetilde{\mathcal{T}} u$ thus the set $\mathcal{H}$ in (6.7) is a (nonempty) compact set in $E$.

Now consider, for each $u \in \mathcal{H}$, the set $C_{u}:=\{z \in \mathcal{H} ; z \leq u$ in $\Omega\}$.

Claim 6.6 The family $\left\{C_{u}\right\}_{u \in \mathcal{H}}$ has the finite intersection property, i.e. the intersection of any finite number of sets $\mathcal{C}_{u}$ is not empty.

Proof. Let $u_{1}, \ldots, u_{\kappa} \in \mathcal{H}$. Observe that $\widetilde{\beta}:=\min _{1 \leq i \leq \kappa} u_{i}$ is an $L^{p}$-viscosity supersolution of $(\mathrm{P})$, with $\alpha \leq \widetilde{\beta} \leq \beta$ in $\Omega$. Furthermore, under hypothesis $\left(H_{2}\right)$, such $\widetilde{\beta}$ is a minimum of strong solutions of $(\mathrm{P})$, which is exactly what we need in order to use part 1 of the above proof, obtaining the existence of a solution $v$ of $(\mathrm{P})$ with $\alpha \leq v \leq \widetilde{\beta} \leq \beta$ in $\Omega$, i.e. $v \in \mathcal{H}$ and $v \leq u_{i}$, for every $i \in\{1, \ldots, \kappa\}$, so $v \in \cap_{1 \leq i \leq \kappa} \mathcal{C}_{u_{i}} \neq \emptyset$.

By the definition of compacity of $\mathcal{H}$ by open covers and claim 6.6, there exists $\underline{u} \in \cap_{u \in \mathcal{H}} \mathcal{C}_{u}$ (see, for example, theorem 26.9 in [88]). But then there exists a solution $\underline{u}$ of $(\mathrm{P})$ with $\alpha \leq \underline{u} \leq u$ in $\Omega$, for all $u \in \mathcal{H}$. Analogously we prove the existence of $\bar{u}$, with $u \leq \bar{u} \leq \beta$ in $\Omega$, for every $u \in \mathcal{H}$.

Remark 6.7 The conclusion of the theorem 6.3 is still true if, instead of $c(x)$, we have some $c(x, u)$ such that $c(x, u) u=c(x) T_{a} u$, where $T_{a}$ is a truncation of $u$, i.e. $T_{a}(u)=u$ for $u \geq a, T_{a}(u)=a$ for $u<a$. In this case, similiar to the "tilde" truncation, $\left|T_{a} u_{k}-T_{a} u\right| \leq\left|u_{k}-u\right|$ and the rest of the proof carries on in the same way.

\section{2}

\section{Some auxiliary results}

We start by constructing an auxiliary problem $\left(P_{\lambda, k}\right)$, for which we can assure that there are no solutions for large $k$, and that $\left(P_{\lambda, 0}\right)$ reduces to the problem $\left(P_{\lambda}\right)$. This is a typical but essential argument (see [66], [15]) that allows us to find a second solution via degree theory, by homotopy invariance in $k$.

Fix $\Lambda_{2}>0$. Recall that proposition 5.7 gives us an a priori lower uniform bound $C_{0}$, depending only on $n, p, \lambda_{P}, \Lambda_{P}, \mu_{1}, \Omega, \Lambda_{2},\|b\|_{L^{\infty}(\Omega)},\|c\|_{L^{\infty}(\Omega)}$ and $\left\|h^{-}\right\|_{L^{\infty}(\Omega)}$, such that

$u \geq-C_{0}$, for all $L^{p}$-visc. supersol. $u$ of $\left(P_{\lambda}\right)$, for all $\lambda \in\left[0, \Lambda_{2}\right]$. 
Consider, thus, the problem

$$
\left\{\begin{aligned}
-F[u] & =\lambda c(x) u+h(x)+\langle M(x) D u, D u\rangle+k \widetilde{c}(x) & & \text { in } \quad \Omega \\
u & =0 & & \text { on } \partial \Omega
\end{aligned} \quad\left(P_{\lambda, k}\right)\right.
$$

for $k \geq 0, \lambda \in\left[0, \Lambda_{2}\right], F$ satisfying $(\mathrm{SC})_{0},\left(H_{\beta}\right)$ and $\left(H_{1}\right), M$ satisfying $(M)$, $c \geqq 0, c, h \in L^{\infty}(\Omega)$ and $\widetilde{c}$ being defined as

$$
\widetilde{c}(x)=\widetilde{c}_{\Lambda_{2}}(x):=A c(x)+h^{-}(x)+\Lambda_{2} C_{0} c(x) \in L_{+}^{\infty}(\Omega),
$$

with $A:=\lambda_{1} / m, m=\mu_{1} / \Lambda_{P}$, where $\lambda_{1}=\lambda_{1}^{+}\left(\mathcal{L}^{-}(c), \Omega\right)>0$ is the first eigenvalue with weight $c$ of the proper operator $\mathcal{L}^{-}$, associated to the positive eigenfunction $\varphi_{1} \in W^{2, p}(\Omega)$, given by proposition 4.3 , that is,

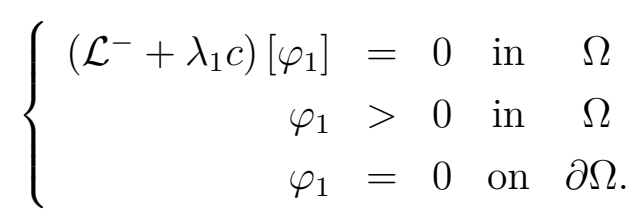

Note that every $L^{p}$-viscosity solution of $\left(P_{\lambda, k}\right)$ is also an $L^{p}$-viscosity supersolution of $\left(P_{\lambda}\right)$, since $k \widetilde{c} \geq 0$, and so satisfies (6.8). From this and (6.9) we have, for all $k \geq 1$ and for a.e. in $\Omega$,

$$
\lambda c(x) u+h(x)+k \widetilde{c}(x) \geq-\Lambda_{2} C_{0} c(x)-h^{-}(x)+\widetilde{c}(x)=A c(x) \supsetneqq 0 .
$$

Lemma 6.8 For each fixed $\Lambda_{2}>0,\left(P_{\lambda, k}\right)$ has no solutions for all $k \geq 1$ and $\lambda \in\left[0, \Lambda_{2}\right]$.

Proof. First observe that every $L^{p}$-viscosity solution of $\left(P_{\lambda, k}\right)$, for $\lambda \in\left[0, \Lambda_{2}\right]$, is positive in $\Omega$. Indeed, from (6.11), $(\mathrm{SC})_{0}$ and $M \geq 0$, we have that $u$ is an $L^{p}$-viscosity solution of

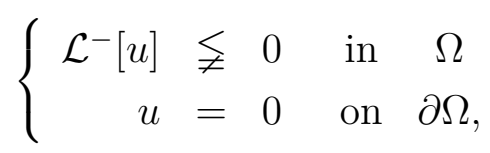

and this implies that $u \geq 0$ in $\Omega$ by ABP. Then $u>0$ in $\Omega$ by SMP.

Assume, in order to obtain a contradiction, that $\left(P_{\lambda, k}\right)$ has a solution $u$. Again by $(M),(6.11)$ and $(\mathrm{SC})_{0}, u$ is also an $L^{p}$-viscosity solution of

$$
\left\{\begin{array}{rlrl}
\mathcal{L}^{-}[u] & \leq-\mu_{1}|D u|^{2}-A c(x) & & \text { in } \Omega \\
u & >0 & \text { in } \Omega
\end{array}\right.
$$


and from lemma 2.20,

$$
\left\{\begin{aligned}
\left(\mathcal{L}^{-}+\lambda_{1} c\right)[v] & \leq-A c(x) & & \text { in } \Omega \\
v & >0 & & \text { in } \Omega
\end{aligned}\right.
$$

using $m A=\lambda_{1}$, where $v=\frac{1}{m}\left\{e^{m u}-1\right\}$, for $m$ and $A$ given by (6.9). Then (6.12) and (6.10), together with proposition 4.4 (see also remark 4.6), yields $v=t \varphi_{1}$ for some $t>0$. But this contradicts the first inequality in (6.12), since $A c(x) \nsupseteq 0$ and $\left(\mathcal{L}^{-}+\lambda_{1} c\right)\left[t \varphi_{1}\right]=t\left(\mathcal{L}^{-}+\lambda_{1} c\right)\left[\varphi_{1}\right]=0$ in $\Omega$.

When we are assuming hypothesis $\left(H_{2}\right)$ we just say solutions to mean strong solutions of $\left(\bar{P}_{\lambda}\right)$. However, sub and supersolutions of such equations, in general, are not assumed strong (since we are considering the problem in the $L^{p}$-viscosity sense), unless specified otherwise. To avoid possible confusion, we always make explicit the notion of sub/supersolution we are referring to.

The next result is important in degree arguments, bearing in mind the set $\mathcal{S}$ in theorem 6.3 .

Lemma 6.9 Suppose $(\mathrm{SC})_{0},\left(H_{\beta}\right)$ and $\left(H_{2}\right)$. Then, for every $\lambda>0$, there exists a strong strict subsolution $\alpha_{\lambda}$ of $\left(P_{\lambda}\right)$ which is strong minimal, in the sense that every strong supersolution $\beta$ of $\left(P_{\lambda}\right)$ satisfies $\alpha_{\lambda} \leq \beta$ in $\Omega$.

When $u_{0}$ has a sign, we will see in the proofs of theorems 5.4 and 5.5 that $u_{0}$ can be taken as $\beta$ and $\alpha$, respectively, in theorem 6.3 for the problem $\left(P_{\lambda}\right)$, for all $\lambda>0$. In the second case, lemma 6.9 will not be necessary.

Proof. Let $K$ be the positive constant from proposition 5.7 such that every $L^{p}$-viscosity supersolution $\beta$ of

$$
\left\{\begin{aligned}
-F[\beta] & \geq \lambda c(x) \beta+\langle M(x) D \beta, D \beta\rangle-h^{-}(x)-1 & \text { in } \Omega \\
\beta & \geq 0 & \text { on } \partial \Omega
\end{aligned}\right.
$$

satisfies $\beta \geq-K$ in $\Omega$. Let $\alpha_{0}$ be the strong solution of the problem

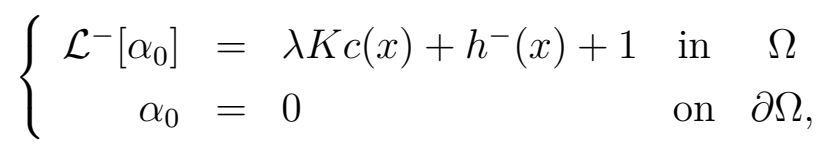

given, for example, by proposition 3.5. Then, as the right hand side of (6.13) is positive, by ABP, SMP and Hopf, we have $\alpha_{0} \ll 0$ in $\Omega$.

Claim 6.10 Every $L^{p}$-viscosity supersolution $\beta$ of $\left(P_{\lambda}\right)$ satisfies $\beta \geq \alpha_{0}$ in $\Omega$.

Proof. First notice that $\beta$ is an $L^{p}$-viscosity supersolution of $\left(\widetilde{P}_{\lambda}\right)$ and so satisfies $\beta \geq-K$. Second, by $(\mathrm{SC})_{0}$ and $M \geq 0, \beta$ is also an $L^{p}$-viscosity 
supersolution of

$$
-\mathcal{L}^{-}[\beta] \geq \lambda c(x) \beta+h(x) \geq-\lambda K c(x)-h^{-}(x)-1 \quad \text { in } \Omega
$$

and setting $v:=\beta-\alpha_{0}$ in $\Omega, v$ is an $L^{p}$-viscosity solution of

$\mathcal{L}^{-}[v] \leq \mathcal{M}^{-}\left(D^{2} \beta\right)+\mathcal{M}^{+}\left(-D^{2} \alpha_{0}\right)-b(x)|D \beta|+b(x)\left|D \alpha_{0}\right|=\mathcal{L}^{-}[\beta]-\mathcal{L}^{-}\left[\alpha_{0}\right] \leq 0$

since $\alpha_{0}$ is strong. Further, $v \geq 0$ on $\partial \Omega$ then, by ABP, $v \geq 0$ in $\Omega$.

Set

$$
\bar{c}(x, t)=\left\{\begin{array}{cc}
c(x) & \text { if } t \geq-K \\
-K c(x) / t & \text { if } t<-K
\end{array}\right.
$$

Note that $0 \leq \bar{c}(x, t) \leq c(x)$ a.e. in $\Omega$ and $\bar{c}(x, t) t \geq-K c(x)$ for $t \in \mathbb{R}$. Then,

$$
\begin{aligned}
-F\left[\alpha_{0}\right] & \leq-\mathcal{L}^{-}\left[\alpha_{0}\right]=-\lambda K c(x)-h^{-}(x)-1 \\
& \leq \lambda \bar{c}\left(x, \alpha_{0}\right) \alpha_{0}+\left\langle M(x) D \alpha_{0}, D \alpha_{0}\right\rangle-h^{-}(x)-1
\end{aligned}
$$

since $M \geq 0$ and $\alpha_{0}$ is a strong subsolution of $\left(\bar{P}_{\lambda}\right)$.

Consider the problem $\left(\bar{P}_{\lambda}\right)$, which we define as the problem $\left(P_{\lambda}\right)$ with $c, h$ replaced by $\bar{c}=\bar{c}(x, u), \bar{h}=-h^{-}-1$. So, solutions of $\left(\bar{P}_{\lambda}\right)$ are strong, by hypothesis $\left(H_{2}\right)$. Moreover, observe that we are in the situation of remark 6.7, since $\bar{c}(x, u) u=c(x) T_{-K} u$ as in there, which allows us to use theorem 6.3 in order to obtain solutions of $\left(\bar{P}_{\lambda}\right)$.

Let $\beta_{0}$ be some fixed strong supersolution of $\left(P_{\lambda}\right)$ (if there were not strong supersolutions of $\left(P_{\lambda}\right)$, the proof is finished). Then, by claim 6.10 , we have $\alpha_{0} \leq \beta_{0}$ in $\Omega$. Also, in that proof we observed that $\beta_{0} \geq-K$, then $\bar{c}\left(x, \beta_{0}\right) \equiv c(x)$ a.e. $x \in \Omega$, which means that

$$
\begin{aligned}
-F\left[\beta_{0}\right] & \geq \lambda c(x) \beta_{0}+\left\langle M(x) D \beta_{0}, D \beta_{0}\right\rangle+h(x) \\
& \geq \lambda \bar{c}(x) \beta_{0}+\left\langle M(x) D \beta_{0}, D \beta_{0}\right\rangle-h^{-}(x)-1
\end{aligned}
$$

and so $\beta_{0}$ is a strong supersolution of $\left(\bar{P}_{\lambda}\right)$. By theorem 6.3 and remark 6.7, we obtain an $L^{p}$-viscosity solution $w$ of this problem, with $\alpha_{0} \leq w \leq \beta_{0}$ in $\Omega$, which is strong and can be chosen as the minimal solution in the order interval $\left[\alpha_{0}, \beta_{0}\right]$, by hypothesis $\left(H_{2}\right)$. 
Remark 6.11 Notice that $\bar{c}(x, t) t \geq c(x) t$ a.e. $x \in \Omega$ for all $t \in \mathbb{R}$, so

$$
\begin{aligned}
-F[w] & =\lambda \bar{c}(x, w) w+\langle M(x) D w, D w\rangle-h^{-}(x)-1 \\
& \geq \lambda c(x) w+\langle M(x) D w, D w\rangle-h^{-}(x)-1
\end{aligned}
$$

a.e. in $\Omega$, that is, $w$ is also a strong supersolution of $\left(\widetilde{P}_{\lambda}\right)$.

Claim 6.12 For every $\beta$ strong supersolution of $\left(P_{\lambda}\right), \beta \geq w$ in $\Omega$.

Proof. Let $\beta$ be any strong supersolution of $\left(P_{\lambda}\right)$. As in the argument above for $\beta_{0}$, we have that $\beta$ is also a strong supersolution of $\left(\bar{P}_{\lambda}\right)$. Suppose that the conclusion is not verified, i.e. that there exists $x_{0} \in \Omega$ such that $\beta\left(x_{0}\right)<w\left(x_{0}\right)$ and define

$$
\beta_{1}:=\min \{w, \beta\} \not \equiv w .
$$

Then $\beta_{1}$ is the minimum of strong supersolutions, hence itself is an $L^{p}$-viscosity supersolution of $\left(\bar{P}_{\lambda}\right)$ and of $\left(\widetilde{P}_{\lambda}\right)$, by remark 6.11 . Following the same lines as in claim 6.10, $\beta_{1} \geq \alpha_{0}$ in $\Omega$. Thus, by theorem 6.3 and remark 6.7, there exists an $L^{p}$-viscosity solution $w_{1}$ of $\left(\bar{P}_{\lambda}\right)$, strong by $\left(H_{2}\right)$, such that $\alpha_{0} \leq w_{1} \leq \beta_{1} \supsetneqq w \leq \beta_{0}$ in $\Omega$, which contradicts the minimality of $w$.

Claim 6.13 $w$ is a strong strict subsolution of $\left(P_{\lambda}\right)$.

Proof. From remark $6.11, w \geq-K$ and $\bar{c}(x, w) \equiv c(x)$, which implies that $w$ actually satisfies (6.14) with equality, from where

$$
-F[w]<\lambda c(x) w+\langle M(x) D w, D w\rangle+h(x) \text { a.e. in } \Omega
$$

with $w=0$ on $\partial \Omega$. Thus $w$ is a strong subsolution of $\left(P_{\lambda}\right)$. What remains to be proved is that $w$ is strict, in order to choose $\alpha_{\lambda}$ as $w$. Therefore, in the sense of definition 6.2 , let $u \in E$ be an $L^{p}$-viscosity supersolution of $\left(P_{\lambda}\right)$ with $u \geq w$ in $\Omega$. Then, since $w$ is strong, $U:=u-w \geq 0$ in $\Omega$ is an $L^{p}$-viscosity supersolution of

$$
-\mathcal{L}^{-}[U] \geq \lambda c(x) U+\langle M(x) D U, D U\rangle+\langle M(x) D U, D w\rangle+\langle M(x) D w, D U\rangle
$$

in $\Omega$, using $(\mathrm{SC})_{0}$ and $0 \leq M(x) \leq \mu_{2}$. Hence, for $\widetilde{b}=b+2 \mu_{2}|D w| \in L_{+}^{p}(\Omega)$, $U$ satisfies

$$
\left\{\begin{aligned}
\mathcal{M}^{-}\left(D^{2} U\right)-\tilde{b}(x)|D U| & <0 \text { in } \Omega \\
U & \geq 0 \text { in } \bar{\Omega}
\end{aligned}\right.
$$

in the $L^{p}$-viscosity sense and by SMP, $U>0$ in $\Omega$. If there exists $x_{0} \in \partial \Omega$ with $U\left(x_{0}\right)=0$, Hopf lemma implies that $\partial_{\nu} U\left(x_{0}\right)>0$. Then, $U \gg 0$ in $\Omega$. 


\section{3}

\section{Proof of Theorem 5.3}

Suppose, at first, $(\mathrm{SC}),\left(H_{\beta}\right)$ and $\left(H_{1}\right)$.

We start proving the first statement in theorem 5.3, about existence of solutions for $\lambda<0$. Set $\alpha:=u_{0}-\left\|u_{0}\right\|_{\infty}$ and $\beta:=u_{0}+\left\|u_{0}\right\|_{\infty}$. Thus, $\alpha, \beta$ is a pair of strong sub and supersolutions of $\left(P_{\lambda}\right)$, for each $\lambda<0$, with $\alpha \leq \beta$ in $\Omega$. Indeed, using (SC), $\alpha \leq u_{0}$ and $0 \leq \lambda c(x) \alpha$, we have

$$
\left\{\begin{array}{rlrl}
-F[\alpha] & \leq-F\left[u_{0}\right] \leq \lambda c(x) \alpha+h(x)+\langle M(x) D \alpha, D \alpha\rangle & & \text { in } \Omega \\
\alpha & \leq u_{0}=0 & \text { on } \partial \Omega
\end{array}\right.
$$

and similarly for $\beta$, with $\beta \geq u_{0}, 0 \geq \lambda c(x) \beta$ and reversed inequalities. Therefore, theorem 6.3 gives us a solution $u_{\lambda} \in[\alpha, \beta]$, for all $\lambda<0$.

Observe that, since $\alpha \leq u_{\lambda} \leq \beta$, we can say that $\left\|u_{\lambda}\right\|_{C^{1, \alpha}(\bar{\Omega})} \leq C$ for all $\lambda \in[0,1]$, by the $C^{1, \alpha}$ estimates (theorem 3.1 and remark 3.3). Thus, take a sequence $\lambda_{k} \leq 0$ with $\lambda_{k} \rightarrow 0$ as $k \rightarrow \infty$. Next, the compact inclusion $C^{1, \alpha}(\bar{\Omega}) \subset E$ gives us some $u \in E$ such that $u_{k} \rightarrow u$ in $E$, up to a subsequence. Hence we can define, for each $\varphi \in W_{\text {loc }}^{2, p}(\Omega)$,

$$
\left\{\begin{aligned}
g_{k}(x) & :=F\left(x, u_{k}, D \varphi, D^{2} \varphi\right)+h(x)+\lambda_{k} c(x) u_{k}, \\
g(x) & :=F\left(x, u, D \varphi, D^{2} \varphi\right)+h(x) .
\end{aligned}\right.
$$

Therefore

$$
\left\|g_{k}-g\right\|_{L^{p}(\Omega)} \leq\left|\lambda_{k}\right|\|c\|_{L^{p}(\Omega)}\left\|u_{0}\right\|_{\infty}+\|d\|_{L^{p}(\Omega)} \omega\left(\left\|u_{k}-u\right\|_{L^{\infty}(\Omega)}\right) \rightarrow 0
$$

as $k \rightarrow \infty$. By proposition 2.12, we have that $u$ is an $L^{p}$-viscosity solution of $\left(P_{0}\right)$. From the uniqueness of the solution at $\lambda=0, u$ needs to be equal to $u_{0}$. Finally, since the sequence of $\lambda$ converging to zero is arbitrary, we obtain $\left\|u_{\lambda}-u_{0}\right\|_{E} \rightarrow 0$ as $k \rightarrow \infty$.

Now we prove the existence of a continuum from $u_{0}$.

Fix an $\varepsilon>0$ and consider another pair of sub and supersolutions given by $\alpha:=u_{0}-\varepsilon$ and $\beta:=u_{0}+\varepsilon$. Analogously to (6.16), we see that $\alpha, \beta$ is a pair of strong sub and supersolutions for $\left(P_{0}\right)$. Notice that they are not a pair for $\left(P_{\lambda}\right)$ with $\lambda<0$, since they do not have a sign. However, $\alpha<u_{0}<\beta$ in $\bar{\Omega}$, which implies that $\alpha \ll u_{0} \ll \beta$ in $\Omega$. Since $u_{0}$ is the unique $L^{p_{\text {-viscosity }}}$ solution of the problem $\left(P_{0}\right)$, then $\alpha, \beta$ are strict in the sense of definition 6.2. Then, theorem 6.3 (i) gives us, for $\mathcal{S}=\mathcal{O} \cap \mathcal{B}_{R}$ defined there, that

$$
\operatorname{deg}\left(I-\mathcal{T}_{0}, \mathcal{S}, 0\right)=1
$$


Using again that $u_{0}$ is the unique $L^{p}$-viscosity solution of $\left(P_{0}\right)$, we have further that $\operatorname{ind}\left(I-\mathcal{T}_{0}, u_{0}\right)=1$. Then, by proposition 4.11 (since $\mathcal{T}_{\lambda}$ is continuous in $\lambda$, uniformly with respect to $u$ in balls of $E$, by stability), there exists a continuum $\mathcal{C} \subset \Sigma$ such that both components

$$
\mathcal{C} \cap([0,+\infty) \times E) \text { and } \mathcal{C} \cap((-\infty, 0] \times E)
$$

are unbounded in $\mathbb{R}^{ \pm} \times E$. This proves item 1 .

From now on, we suppose $(\mathrm{SC})_{0}$.

Let us prove point 2. in theorem 5.3. The continuum $\mathcal{C} \subset \Sigma$ is such that its projection on the $\lambda$-axis is either $\mathbb{R}$ (and we obtain $(i i)$ in theorem 5.3) or it is $(-\infty, \bar{\lambda}]$, with $0<\bar{\lambda}<+\infty$. In the second case, since we know that the component $\mathcal{C}^{+}$is unbounded in $R^{+} \times E$, its projection on the $E$ axis must be unbounded in $E$.

Under $(\mathrm{SC})_{0}$, by theorem 5.6, for any $0<\Lambda_{1}<\Lambda_{2}$ there is an $L^{\infty}$ a priori bound for the solutions of $\left(P_{\lambda}\right)$, for all $\lambda \in\left[\Lambda_{1}, \Lambda_{2}\right]$. Then, by $C^{1, \alpha}$ global estimate (theorem 3.1), we have also a $C^{1, \alpha}$ a priori bound for these solutions i.e. the projection of $\mathcal{C}^{+} \cap\left(\left[\Lambda_{1}, \Lambda_{2}\right] \times E\right)$ on $E$ is bounded. So, $\mathcal{C}^{+}$needs to be unbounded in $E$ when we approach $\lambda=0$ from the right.

Now, by proposition 5.7 , there is a lower $L^{\infty}$ bound for the solutions, for every $\lambda \leq \Lambda_{2}$. Therefore, $\mathcal{C}^{+}$must emanate from plus infinity to the right of $\lambda=0$, with the positive part of its solutions blowing up to infinity in $C(\bar{\Omega})$. Thus, $(i)$ and $(i i)$ in 2. are proved.

Now we pass to the multiplicity results in item 3. of theorem 5.3.

Observe that, up to taking a larger $R$ in (6.18), by $C^{1, \alpha}$ estimates we can suppose that for every $u, L^{p}$-viscosity solution of $\left(P_{\lambda}\right)$ in $\left[u_{0}-\varepsilon, u_{0}+\varepsilon\right]$,

$$
\|u\|_{C^{1, \alpha}(\bar{\Omega})}<R, \quad \text { for all } \lambda \in[0,1] .
$$

Claim 6.14 There exists a $\lambda_{0}>0$ such that

$$
\operatorname{deg}\left(I-\mathcal{T}_{\lambda}, \mathcal{S}, 0\right)=1, \quad \text { for all } \lambda \in\left(0, \lambda_{0}\right)
$$

Proof. Let us prove the existence of a $\lambda_{0}>0$ such that, for all $\lambda \in\left(0, \lambda_{0}\right)$, $\left(P_{\lambda}\right)$ has no solution on $\partial \mathcal{S}$. Suppose not, i.e. that for all $\lambda_{0}>0$, there exists a $\lambda \in\left(0, \lambda_{0}\right)$ such that $\mathcal{T}_{\lambda}$ has a fixed point on $\partial \mathcal{S}$. Then for every $k \in \mathbb{N}$, there exists $\lambda_{k} \in\left(0, \frac{1}{k}\right)$ and $u_{k} \in \partial \mathcal{S}$ a solution of $\left(P_{\lambda_{k}}\right)$. By $(6.19), u_{k} \notin \partial \mathcal{B}_{R}$ and so $u_{k} \in \partial \mathcal{O}$, for all $k \in \mathbb{N}$. Note that

$$
\partial \mathcal{O}=\left\{u \in C_{0}^{1}(\bar{\Omega}) ; \alpha \leq u \leq \beta \text { in } \bar{\Omega} \text { and } u \text { "touches" } \alpha \text { or } \beta\right\}
$$


where "touches" in the above, as in [66], has the following meaning.

Definition 6.15 Let $u, v \in C_{0}^{1}(\bar{\Omega})$. We say that $u$ touches $v$ if there exists $x \in \Omega$ with $u(x)=v(x)$ or if there exists $x \in \partial \Omega$ with $\partial_{\nu} u(x)=\partial_{\nu} v(x)$. In any case, $u(x)=v(x)$ at a point $x \in \bar{\Omega}$.

If $u_{k}$ touches $\alpha$, there exists a $x \in \bar{\Omega}$ such that $u_{k}(x)=u_{0}(x)-\varepsilon$, and since $u_{k} \geq u_{0}-\varepsilon$, then $\max _{\bar{\Omega}}\left(u_{0}-u_{k}\right)=\varepsilon$. If on the other side $u_{k}$ touches $\beta$, there exists a $x \in \bar{\Omega} ; u_{k}(x)=u_{0}(x)+\varepsilon$, and since $u_{k} \leq u_{0}+\varepsilon$, then $\max _{\bar{\Omega}}\left(u_{k}-u_{0}\right)=\varepsilon$. Anyway,

$$
\left\|u_{k}-u_{0}\right\|_{\infty}=\max _{\bar{\Omega}}\left|u_{k}-u_{0}\right|=\varepsilon, \quad \text { for all } k \in \mathbb{N} .
$$

By (6.19) and compact inclusion $C^{1, \alpha}(\bar{\Omega}) \subset E$, there exists $u \in E$ such that $u_{k} \rightarrow u$ in $E$ as $k \rightarrow \infty$, up to taking a subsequence. Hence, by stability (proposition 2.12), $u$ is an $L^{p}$-viscosity solution of $\left(P_{0}\right)$. Indeed, we define $g$ and $g_{k}$ as in (6.17), for each $\varphi \in W_{l o c}^{2, p}(\Omega)$, from where

$$
\left\|g_{k}-g\right\|_{L^{p}(\Omega)} \leq \lambda_{k}\|c\|_{L^{p}(\Omega)}\left(\left\|u_{0}\right\|_{\infty}+\varepsilon\right) \rightarrow 0 \text { as } k \rightarrow \infty .
$$

By uniqueness in $\lambda=0, u=u_{0}$, which contradicts (6.20) by passing to limits.

Therefore, the following degree is well defined and by the homotopy invariance and (6.18) we obtain

$$
\operatorname{deg}\left(I-\mathcal{T}_{\lambda}, \mathcal{S}, 0\right)=\operatorname{deg}\left(I-\mathcal{T}_{0}, \mathcal{S}, 0\right)=1 \text {, for all } \lambda \in\left(0, \lambda_{0}\right)
$$

Claim 6.16 $\left(P_{\lambda}\right)$ has two solutions when $\lambda \in\left(0, \lambda_{0} / 2\right]$.

Proof. The existence of a first solution $u_{\lambda, 1}$ with $u_{0}-\varepsilon \ll u_{\lambda, 1} \ll u_{0}+\varepsilon$ is ensured by claim 6.14. Set $\Lambda_{2}:=\lambda_{0} / 2$. Then, lemma 6.8 implies that $\left(P_{\lambda, k}\right)$ has no solutions for $k \geq 1$ and $\lambda \in\left(0, \Lambda_{2}\right]$.

Fix a $\lambda \in\left(0, \Lambda_{2}\right]$. With $h$ replaced by $h+k \widetilde{c}$ (see (6.9)) we have, by theorem 5.6, an $L^{\infty}$ a priori bound for solutions of $\left(P_{\lambda, k}\right)$, for every $k \in[0,1]$. Precisely, we get an $L^{\infty}$ a priori bound for solutions of $\left(P_{\mu, k}\right)$, for all $\mu \in\left[\lambda, \Lambda_{2}\right]$, depending on $\lambda$ and $\Lambda_{2}$. This provides, by the $C^{1, \alpha}$ estimates (theorem 3.1), an a priori bound for solutions in $E$, namely

$$
\|u\|_{E}<R_{0} \text {, for every } u L^{p} \text {-viscosity solution of }\left(P_{\lambda, k}\right) \text {, for all } k \in[0,1] \text {, }
$$

and for some $R_{0}>R$ that depends, in addition to the coefficients of the equation, also on $\lambda$ and the $L^{p}$-norm of $\widetilde{c}$. By the homotopy invariance of the 
degree in $k \in[0,1]$ and the fact that, for $k=1,\left(P_{\lambda, k}\right)$ has no solution,

$$
\operatorname{deg}\left(I-\mathcal{T}_{\lambda}, \mathcal{B}_{R_{0}}, 0\right)=\operatorname{deg}\left(I-\mathcal{T}_{\lambda, 0}, \mathcal{B}_{R_{0}}, 0\right)=\operatorname{deg}\left(I-\mathcal{T}_{\lambda, 1}, \mathcal{B}_{R_{0}}, 0\right)=0
$$

where $\mathcal{T}_{\lambda, k}$ is the operator $\mathcal{T}_{\lambda}$ in which we replace $h$ by $h+k \widetilde{c}$ (of course $\mathcal{T}_{\lambda, k}$ is still completely continuous). But then, by the excision property of the degree,

$$
\operatorname{deg}\left(I-\mathcal{T}_{\lambda}, \mathcal{B}_{R_{0}} \backslash \mathcal{S}, 0\right)=\operatorname{deg}\left(I-\mathcal{T}_{\lambda}, \mathcal{B}_{R_{0}}, 0\right)-\operatorname{deg}\left(I-\mathcal{T}_{\lambda}, \mathcal{S}, 0\right)=-1
$$

which provides the second solution $u_{\lambda, 2} \in \mathcal{B}_{R_{0}} \backslash \mathcal{S}$ that we were looking for.

Next, by claim 6.16 , the quantity

$$
\bar{\lambda}:=\sup \left\{\mu ; \forall \lambda \in(0, \mu),\left(P_{\lambda}\right) \text { has at least two solutions }\right\} \in(0,+\infty]
$$

it is well defined and greater or equal than $\lambda_{0} / 2$.

Claim 6.17 $u_{\lambda, 1} \rightarrow u_{0}$ in $E$ and $\max _{\bar{\Omega}} u_{\lambda, 2} \rightarrow+\infty$ as $\lambda \rightarrow 0^{+}$.

Proof. Let $\left(\lambda_{k}\right)_{k \in \mathbb{N}} \subset(0, \bar{\lambda})$ be a decreasing sequence with $\lambda_{k} \rightarrow 0$. Say $\lambda_{k} \leq \lambda_{0} / 2$ for $k \geq k_{0}$. Since $u_{\lambda_{k}, 1} \in \mathcal{S}, u_{0}-\varepsilon \leq u_{\lambda_{k}, 1} \leq u_{0}+\varepsilon$ in $\bar{\Omega}$, therefore it is bounded in $C^{1, \alpha}(\bar{\Omega})$ by theorem 3.1. Hence, exactly as in (6.17), we show by stability that $u_{\lambda_{k}, 1} \rightarrow u$ in $E$, where $u$ is a solution of $\left(P_{0}\right)$. Then $u=u_{0}$.

If, in turn, the respective sequence $u_{\lambda_{k}, 2}$ were uniformly bounded from above, it would be unifomly bounded in $C(\bar{\Omega})$ using proposition 5.7 , so bounded in $C^{1, \alpha}(\bar{\Omega})$ and the paragraph above would imply that $u_{\lambda_{k}, 2} \rightarrow u_{0}$ in $E$. Since $u_{0} \in \mathcal{S}$ and $\mathcal{S}$ is open in $E$, then $u_{\lambda_{k}, 2}$ should belong to $\mathcal{B}_{r}^{E}\left(u_{0}\right) \subset \mathcal{S}$ for large $k$, for some $r>0$. But this contradicts the fact that $u_{\lambda_{k}, 2} \notin \mathcal{S}$.

Claim 6.18 In case $\bar{\lambda}<+\infty$, the problem $\left(P_{\bar{\lambda}}\right)$ has at least one solution.

Proof. Let $\lambda_{k} \in(0, \bar{\lambda})$ be such that $\lambda_{k} \rightarrow \bar{\lambda}$ and let $u_{k}$ be a sequence of solutions for $\left(P_{\lambda_{k}}\right)$. Say $\lambda_{k} \in[\bar{\lambda} / 2, \bar{\lambda}]$ for $k \geq k_{0}$. This provides an $L^{\infty}$ a priori bound for $u_{k}$, by theorem 5.6, i.e. $\left\|u_{k}\right\|_{\infty} \leq C_{1}$, which implies that $\left\|u_{k}\right\|_{C^{1, \alpha}(\bar{\Omega})} \leq C_{2}$. Again, by compact inclusion and stability, we obtain $u_{k} \rightarrow u$ in $E$, where $u$ is a solution of $\left(P_{\bar{\lambda}}\right)$. Surely, for stability we need to consider, this time, $g(x):=F\left(x, u, D \varphi, D^{2} \varphi\right)+\bar{\lambda} c(x) u+h(x)$ and so $\left\|g_{k}-g\right\|_{L^{p}(\Omega)} \leq\left(\bar{\lambda}-\lambda_{k}\right)\|c\|_{L^{p}(\Omega)} C_{2}+\bar{\lambda}\|c\|_{L^{p}(\Omega)}\left\|u_{k}-u\right\|_{\infty} \rightarrow 0$ as $k \rightarrow \infty$.

To finish the proof of theorem 5.3, it remains to show the last statements in item 4. concerning ordering and uniqueness considerations, in which we assume $\left(H_{2}\right)$. Notice that this automatically implies that solutions $u_{\lambda, 1}$ and $u_{\lambda, 2}$ are strong, as well as every $L^{p}$-viscosity solution of $\left(P_{\lambda}\right)$. 
Further, note that $\left(H_{2}\right)$ provided existence of minimal and maximal solutions on the order interval $[\alpha, \beta]$ in theorem 6.3. Such existence of minimal solution made it possible to find a minimal strong subsolution $\alpha_{\lambda}$ of $\left(P_{\lambda}\right)$ in lemma 6.9 .

Claim 6.19 $u_{\lambda, 1} \ll u_{\lambda, 2}$, for all $\lambda \in(0, \bar{\lambda})$.

Proof. Fix a $\lambda \in(0, \bar{\lambda})$ and consider the strict strong subsolution $\alpha=\alpha_{\lambda}$ given by lemma 6.9. Since in particular $\alpha \leq u$ for every (strong) solution of $\left(P_{\lambda}\right)$, we can choose $u_{\lambda, 1}$ as the minimal strong solution such that $u_{\lambda, 1} \geq \alpha$ in $\Omega$. This choice implies that

$$
u_{\lambda, 1} \supsetneqq u_{\lambda, 2} \text { in } \Omega \text {. }
$$

Indeed, $u_{\lambda, 1} \neq u_{\lambda, 2}$ and, if there would exist a point $x_{0} \in \Omega$ such that $u_{\lambda, 1}\left(x_{0}\right)>u_{\lambda, 2}\left(x_{0}\right)$, by defining $u_{\lambda}:=\min \left\{u_{\lambda, 1}, u_{\lambda, 2}\right\}$, as the minimum of strong supersolutions greater or equal than $\alpha$, so $u_{\lambda} \geq \alpha$ in $\Omega$. Therefore, theorem 6.3 would give us a solution $u$ of $\left(P_{\lambda}\right)$ such that $\alpha \leq u \leq u_{\lambda} \supsetneqq u_{\lambda, 1}$, which contradicts the minimality of $u_{\lambda, 1}$ and implies (6.21).

To finish the proof, define $v:=u_{\lambda, 2}-u_{\lambda, 1} \supsetneqq 0$ in $\Omega$ by (6.21). Then, since $u_{\lambda, 1}$ and $u_{\lambda, 2}$ are strong, $v$ satisfies, almost everywhere in $\Omega$,

$$
\begin{aligned}
-\mathcal{L}^{-}[v] & \geq-F\left[u_{\lambda, 2}\right]+F\left[u_{\lambda, 1}\right] \\
& =\lambda c(x) v+\langle M(x) D v, D v\rangle+\left\langle M(x) D v, D u_{\lambda, 1}\right\rangle+\left\langle M(x) D u_{\lambda, 1}, D v\right\rangle \\
& \geq-2 \mu_{2}\left|D u_{\lambda, 1}\right||D v| .
\end{aligned}
$$

Hence, $v$ is a nonnegative strong solution of $\mathcal{M}^{-}\left(D^{2} v\right)-\widetilde{b}(x)|D v| \leq 0$ in $\Omega$, for $\widetilde{b}=b+2 \mu_{2}\left|D u_{\lambda, 1}\right| \in L_{+}^{p}(\Omega)$. Then SMP gives us that $v>0$ in $\Omega$, since $v \not \equiv 0$. Now, Hopf lemma and $v=0$ on $\partial \Omega$ imply $\left.\partial_{\nu} v\right|_{\partial \Omega}>0$, so $v \gg 0$ in $\Omega$.

As far as uniqueness is concerned, from theorem 1(iii) in [12], if the coercive problem for $\lambda \leq 0$ has a strong solution $u_{\lambda}$, it is the unique $L^{p}$-viscosity solution of $\left(P_{\lambda}\right)$. So, under $\left(H_{2}\right), u_{\lambda}$ is strong, then unique, in the $L^{p}$-viscosity sense, for all $\lambda<0$. Observe that, in this case, we must have $\left\{\left(\lambda, u_{\lambda}\right), \lambda \leq 0\right\} \subset \mathcal{C}$. In other words, the projection of $\mathcal{C}$ on the $\lambda$-axis contains $(-\infty, 0]$, as in theorems 1.1 and 1.2 in [13] for the Laplacian.

We finish the proof of 4 . with the following claim.

Claim 6.20 If $\bar{\lambda}<+\infty$ and $F$ is convex in $(r, p, X)$, the solution $u_{\bar{\lambda}}$ of $\left(P_{\bar{\lambda}}\right)$, obtained in claim 6.18, is unique. 
Proof. Suppose, in order to obtain a contradiction, that there exist two different solutions $u_{1}$ and $u_{2}$ of $\left(P_{\bar{\lambda}}\right)$, both strong by $\left(H_{2}\right)$. Consider $\beta=\beta_{\bar{\lambda}}$ defined as $\frac{1}{2}\left(u_{1}+u_{2}\right)$. Then, a.e. in $\Omega$,

$$
\begin{aligned}
-F[\beta] & \geq-\left\{F\left[u_{1}\right]+F\left[u_{2}\right]\right\} / 2 \\
& =\bar{\lambda} c(x) \beta+h(x)+\left\{\left\langle M(x) D u_{1}, D u_{1}\right\rangle+\left\langle M(x) D u_{2}, D u_{2}\right\rangle\right\} / 2 \\
& \supsetneqq \bar{\lambda} c(x) \beta+h(x)+\langle M(x) D \beta, D \beta\rangle
\end{aligned}
$$

using also the convexity of $p \mapsto\langle M(x) p, p\rangle$. Hence $\beta$ is a strong supersolution of $\left(P_{\bar{\lambda}}\right)$ which is not a solution. Let us see that it is strict. Set $U:=\beta-u$, where $u \in E$ is an $L^{p}$-viscosity subsolution of $\left(P_{\bar{\lambda}}\right)$ with $u \leq \beta$ in $\Omega$. Thus, $U$ is an $L^{p}$-viscosity solution of

$$
\begin{aligned}
-\mathcal{L}^{-}[U] & \supsetneqq \bar{\lambda} c(x) U-\langle M(x) D U, D U\rangle+\langle M(x) D U, D \beta\rangle+\langle M(x) D \beta, D U\rangle \\
& \geq-\mu_{2}|D U|^{2}-2 \mu_{2}|D \beta||D U|,
\end{aligned}
$$

and so, by lemma 2.20 , the function $w:=\frac{1}{m}\left(1-e^{-m U}\right)$, where $m=\mu_{2} / \lambda_{P}$, is a nonnegative $L^{p}$-viscosity solution of $\mathcal{M}^{-}\left(D^{2} w\right)-\widetilde{b}(x)|D w| \supsetneqq 0$ in $\Omega$, with $\widetilde{b}=b+2 \mu_{2}|D \beta| \in L_{+}^{p}(\Omega)$. Then SMP gives us $w>0$ in $\Omega$. Now, Hopf and $w \geq 0$ on $\partial \Omega$ imply that $\left.\partial_{\nu} v\right|_{\partial \Omega}>0$ in the boundary points where $w=0$, and so $w \gg 0$ on $\Omega$. Consequently, $U \gg 0$ in $\Omega$ and therefore $\beta$ is a strict strong supersolution of $\left(P_{\bar{\lambda}}\right)$.

Consider also $\alpha=\alpha_{\bar{\lambda}}$ the strict strong subsolution of $\left(P_{\bar{\lambda}}\right)$ given by proposition 6.9 and look at the set $\overline{\mathcal{O}}=\{\alpha \ll u \ll \beta\}=\left\{\alpha_{\bar{\lambda}} \ll u \ll \beta_{\bar{\lambda}}\right\}$. Again, by the $C^{1, \alpha}$ estimates in chapter 3 ,

$$
\|u\|_{C^{1, \alpha}(\bar{\Omega})} \leq C \text { for all } u \in[\alpha, \beta] L^{p} \text {-visc. sol. of }\left(P_{\lambda}\right), \lambda \in[\bar{\lambda}, \bar{\lambda}+1]
$$

for some $C>0$ that depends on the $L^{\infty}$-norm of $\alpha$. Then, by theorem 6.3, we obtain $R>C$ such that $\operatorname{deg}\left(I-\mathcal{T}_{\bar{\lambda}}, \overline{\mathcal{S}}, 0\right)=1$, where $\overline{\mathcal{S}}=\overline{\mathcal{O}} \cap \mathcal{B}_{R}$.

We claim that there exists $\varepsilon>0$ such that

$$
\operatorname{deg}\left(I-\mathcal{T}_{\lambda}, \overline{\mathcal{S}}, 0\right)=1, \quad \text { for all } \lambda \in[\bar{\lambda}, \bar{\lambda}+\varepsilon]
$$

As in the proof of claim 6.14 , we will verify that there exists some $\varepsilon \in(0,1)$ such that there is no fixed points of $\mathcal{T}_{\lambda}$ on the boundary of $\overline{\mathcal{S}}$, for all $\lambda$ in the preceding interval. Indeed, if this were not the case, there would exist a sequence $\lambda_{k} \rightarrow \bar{\lambda}$ with the respective solutions $u_{k}$ of $\left(P_{\lambda_{k}}\right)$ belonging to $\partial \overline{\mathcal{S}}$. Say $\lambda_{k} \in[\bar{\lambda}, \bar{\lambda}+1]$ for $k \geq k_{0}$. Then, since $\alpha \leq u_{k} \leq \beta$ in $\Omega$, by (6.22) we must 
have $u_{k} \in \partial \overline{\mathcal{O}}$ for $k \geq k_{0}$, which means that for each such $k$,

$$
\max _{\bar{\Omega}}\left(\alpha-u_{k}\right)=0 \text { or } \max _{\bar{\Omega}}\left(u_{k}-\beta\right)=0 .
$$

By (6.22) and the compact inclusion $C^{1, \alpha}(\bar{\Omega}) \subset E, u_{k} \rightarrow u$ in $E$ for some $u \in E$, up to a subsequence. This $u$ is an $L^{p}$-viscosity solution of $\left(P_{\bar{\lambda}}\right)$ by stability (proposition 2.12); and $\alpha \leq u \leq \beta$ in $\Omega$ by taking the limit as $k \rightarrow+\infty$ in the corresponding inequalities for $u_{k}$. Thus $\alpha \ll u \ll \beta$ in $\Omega$, since $\alpha$ and $\beta$ are strict. Passing to limits in (6.24), we obtain that $u$ touches $\alpha$ or $\beta$, which contradicts the definition of $\alpha \ll u \ll \beta$.

Hence, obtaining (6.23) it is just a question of applying homotopy invariance in $\lambda$ in the interval $[\bar{\lambda}, \bar{\lambda}+\varepsilon]$. Next, with (6.23) at hand, we repeat exactly the same argument done in claim 6.16 to obtain the existence of a second solution $u_{\lambda, 2}$ of $\left(P_{\lambda}\right)$, for all $\lambda \in[\bar{\lambda}, \bar{\lambda}+\varepsilon]$. But this, finally, contradicts the definition of $\bar{\lambda}$.

\section{4}

\section{Proof of Theorem 5.4}

Suppose $u_{0} \leq 0$ with $c u_{0} \supsetneqq 0$ in $\Omega$ and $\left(H_{2}\right)$.

Claim 6.21 $u_{0}$ is a strict strong supersolution of $\left(P_{\lambda}\right)$, for all $\lambda>0$.

Proof. Since $\lambda c(x) u_{0} \supsetneqq 0$ in $\Omega, u_{0}$ is a strong supersolution of $\left(P_{\lambda}\right)$ which is not a solution. To see that it is strict, we take $u \in E$ an $L^{p}$-viscosity subsolution of $\left(P_{\lambda}\right)$ such that $u \leq u_{0}$ in $\Omega$, and set $U:=u_{0}-u$. Then, since $u_{0}$ is strong, $U$ is an $L^{p}$-viscosity supersolution of

$$
\begin{aligned}
-\mathcal{L}^{-}[U] & \geq \lambda c(x) U-\langle M(x) D U, D U\rangle+\left\langle M(x) D u_{0}, D U\right\rangle+\left\langle M(x) D u_{0}, D U\right\rangle \\
& \geq-\mu_{2}|D U|^{2}-2 \mu_{2}\left|D u_{0}\right||D U| .
\end{aligned}
$$

Moreover, $\mathcal{M}^{-}\left(D^{2} w\right)-\widetilde{b}(x)|D w| \leq 0$ in $\Omega$ in the $L^{p}$-viscosity sense, where $\widetilde{b}=b+2 \mu_{2}\left|D u_{0}\right| \in L_{+}^{p}(\Omega)$ and $w=\frac{1}{m}\left\{1-e^{-m U}\right\}, m=\mu_{2} / \lambda_{P}$, by lemma 2.20 . Using SMP and the fact that $u_{0}$ is not a solution of $\left(P_{\lambda}\right)$, we have $w>0$ in $\Omega$. Since $w \geq 0$ on $\partial \Omega$, at points belonging to $\partial \Omega$ such that $w>0$ we are done. If in turn $x_{0} \in \partial \Omega$ is such that $w\left(x_{0}\right)=0$, then $\partial_{\nu} U\left(x_{0}\right)>0$ by Hopf. Thus $w \gg 0$ and so $U \gg 0$ in $\Omega$.

We now prove that for all $\lambda>0,\left(P_{\lambda}\right)$ has at least two solutions, $u_{\lambda, 1}$ and $u_{\lambda, 2}$, with $u_{\lambda, 1} \ll u_{0}$ and $u_{\lambda, 1} \ll u_{\lambda, 2}$.

Fix a $\lambda>0$. From lemma 6.9 and claim 6.21, we get a pair of strong strict sub and supersolutions, $\alpha=\alpha_{\lambda}$ and $u_{0}$, which implies, by theorem 6.3 , the existence of a first solution $u_{\lambda, 1} \in \mathcal{S}$, where 


$$
\mathcal{S}=\mathcal{S}_{\lambda}=\left\{u \in C_{0}^{1}(\bar{\Omega}) ; \alpha \ll u \ll u_{0} \text { in } \Omega\right\} \cap \mathcal{B}_{R} \text { for some } R>0
$$

Remark 6.22 We already know, from theorem 5.3, that at least two solutions exist. Here we redefine, as in [15], the set $\mathcal{S}$ in order to obtain more precise informations about $\Sigma$. Notice that, with this new definition, we automatically have $u_{\lambda, 1} \ll u_{0} \leq 0$.

Fix a $\lambda>0$ and set $\Lambda_{2}:=2 \lambda$. As in the proof of claim 6.16 , we observe that, by lemma $6.8,\left(P_{\lambda, k}\right)$ has no solutions for $k \geq 1$. Moreover, for $h$ replaced by $h+k \widetilde{c}$, theorem 5.6 gives us an $L^{\infty}$ a priori bound for solutions of $\left(P_{\lambda, k}\right)$ for every $k \in[0,1]$, which depends on $\lambda$. This provides, by the $C^{1, \alpha}$ global estimates (theorem 3.1), an a priori bound for solutions in $E$, i.e. $\|u\|_{E}<R_{0}$ for every $u$ solution of $\left(P_{\lambda, k}\right)$, for all $k \in[0,1]$, where $R_{0}>R$ also depends on $\lambda$. By the homotopy invariance of the degree,

$$
\operatorname{deg}\left(I-\mathcal{T}_{\lambda}, \mathcal{B}_{R_{0}}, 0\right)=\operatorname{deg}\left(I-\mathcal{T}_{\lambda, 0}, \mathcal{B}_{R_{0}}, 0\right)=\operatorname{deg}\left(I-\mathcal{T}_{\lambda, 1}, \mathcal{B}_{R_{0}}, 0\right)=0
$$

Therefore, by the excision property of the degree

$$
\operatorname{deg}\left(I-\mathcal{T}_{\lambda}, \mathcal{B}_{R_{0}} \backslash \mathcal{S}, 0\right)=\operatorname{deg}\left(I-\mathcal{T}_{\lambda}, \mathcal{B}_{R_{0}}, 0\right)-\operatorname{deg}\left(I-\mathcal{T}_{\lambda}, \mathcal{S}, 0\right)=-1
$$

and the existence of a second solution $u_{\lambda, 2} \in \mathcal{B}_{R_{0}} \backslash \mathcal{S}$ is derived.

Since the argument above can be done for any $\lambda>0$, we obtain the existence of at least two solutions for every positive $\lambda$. Exactly the same reasoning from claims 6.19 and 6.17 applies to check that $u_{\lambda, 1} \ll u_{\lambda, 2}$ in $\Omega$ and to get their behavior when $\lambda \rightarrow 0^{+}$, respectively, since $\bar{\lambda}$ is the same from theorem 5.3. Of course, here $\bar{\lambda}=+\infty$.

Claim 6.23 For $\lambda_{1}<\lambda_{2}$, we have $u_{\lambda_{2}, 1} \ll u_{\lambda_{1}, 1}$ in $\Omega$.

Proof. For fixed $\lambda_{1}<\lambda_{2}$ note that $\lambda_{1} c(x) u_{\lambda_{1}, 1} \supsetneqq \lambda_{2} c(x) u_{\lambda_{1}, 1}$ since $u_{\lambda, 1}<0$. Then, $u_{\lambda_{1}, 1}$ is a strong supersolution of $\left(P_{\lambda_{2}}\right)$ which is not a solution and, in particular, $u_{\lambda_{1}, 1} \neq u_{\lambda_{2}, 1}$.

We first infer that $u_{\lambda_{2}, 1} \supsetneqq u_{\lambda_{1}, 1}$ in $\Omega$. In fact, similarly to the argument in the proof of claim 6.19 , recall that $\alpha=\alpha_{\lambda_{2}}$, given by lemma 6.9 , is such that $\alpha \leq u$ for every strong supersolution of $\left(P_{\lambda_{2}}\right)$, and in particular $\alpha \leq u_{\lambda_{1}, 1}$. Remember also that $u_{\lambda_{2}, 1}$ is the minimal strong solution such that $u_{\lambda_{2}, 1} \geq \alpha$ in $\Omega$. Now, if there was a point $x_{0} \in \Omega$ such that $u_{\lambda_{2}, 1}\left(x_{0}\right)>u_{\lambda_{1}, 1}\left(x_{0}\right)$, by defining $\beta:=\min \left\{u_{\lambda_{1}, 1}, u_{\lambda_{2}, 1}\right\}$, as the minimum of strong supersolutions of $\left(P_{\lambda_{2}}\right)$ not less than $\alpha$, we have $\alpha \leq \beta$ in $\Omega$. Thus, theorem 6.3 provides a solution $u$ of $\left(P_{\lambda_{2}}\right)$ such that $\alpha \leq u \leq \beta \supsetneqq u_{\lambda_{2}, 1}$ in $\Omega$, contradicting the minimality of $u_{\lambda_{2}, 1}$. 
Proceeding as usual, the function $v:=u_{\lambda_{1}, 1}-u_{\lambda_{2}, 1}$ is a nonnegative strong supersolution of $\mathcal{M}^{-}\left(D^{2} v\right)-\widetilde{b}(x)|D v| \leq 0$ in $\Omega$, then SMP gives us that $v>0$ in $\Omega$, since $v \not \equiv 0$. Next, Hopf and $v=0$ on $\partial \Omega$ imply that $\left.\partial_{\nu} v\right|_{\partial \Omega}>0$, hence $v \gg 0$ in $\Omega$.

Remark 6.24 Notice that $u \ll u_{0}$ in $\Omega$, for every nonpositive $L^{p}$-viscosity subsolution $u$ of $\left(P_{\lambda}\right)$ in $E$. Indeed, since $\lambda c(x) u \leq 0$ in $\Omega, u$ is also an $L^{p}$-viscosity subsolution of $\left(P_{0}\right)$. By remark 5.9 we have $u \leq u_{0}$, since $u_{0}$ is strong. Now, by claim 6.21 and definition of strict supersolution, we get $u \ll u_{0}$ in $\Omega$. In particular, $u \equiv 0$ is never a solution of $\left(P_{\lambda}\right)$, for any $\lambda>0$ (recall that $\left.c u_{0} \neq \equiv 0\right)$.

Claim 6.25 In addition to the hypotheses of theorem 5.4, suppose that $F$ is convex in $(r, p, X)$. Then, $\left(P_{\lambda}\right)$ has at most one nonpositive solution. In particular, $\max _{\bar{\Omega}} u_{\lambda, 2}>0$.

Proof. Suppose, in order to obtain a contradiction, that there exist two different nonpositive solutions $u_{1}$ and $u_{2}$, strong by $\left(H_{2}\right)$. By remark 6.24 we know that $u_{1} \ll u_{0}$ and $u_{2} \ll u_{0}$ in $\Omega$. We can assume that they are ordered, in the sense that $u_{1} \supsetneqq u_{2}$. Indeed, observe that $\max \left\{u_{1}, u_{2}\right\} \leq u_{0}$, then theorem 6.3 yields a solution $u_{3}$ of $\left(P_{\lambda}\right)$ with $u_{2} \leq \max \left\{u_{1}, u_{2}\right\} \leq u_{3} \leq u_{0} \leq 0$ in $\Omega$. Thus, if the solutions $u_{1}$ and $u_{2}$ do not satisfy $u_{1} \supsetneqq u_{2}$, then there is a point $x_{0} \in \Omega$ with $u_{1}\left(x_{0}\right)>u_{2}\left(x_{0}\right)$, which implies that $u_{2} \not \equiv \max \left\{u_{1}, u_{2}\right\}$ and so $u_{2} \supsetneqq u_{3}$; in this case we just replace $u_{1}, u_{2}$ by $u_{2}, u_{3}$ respectively.

Since $u_{2} \ll 0$ (from $u_{2} \ll u_{0}$ and $\partial_{\nu} u_{0} \leq 0$ ), the quantity

$$
\tau:=\inf \left\{t>0 ;(1+t) u_{2} \leq u_{1} \text { in } \Omega\right\}
$$

is well defined and finite. Further, $\tau>0$, since $u_{2}-u_{1} \supsetneqq 0$, so this infimum is attained. Then, by setting $w:=\frac{1}{\tau}\left\{(1+\tau) u_{2}-u_{1}\right\}$, we have that $w \leq 0$ satisfies $u_{2}=\frac{\tau}{1+\tau} w+\frac{1}{1+\tau} u_{1}$ and it is a strong subsolution of

$$
\begin{aligned}
F[w] & \geq \frac{1+\tau}{\tau} F\left[u_{2}\right]-\frac{1}{\tau} F\left[u_{1}\right]=\frac{1+\tau}{\tau}\left\{\lambda c(x) u_{2}+\left\langle M(x) D u_{2}, D u_{2}\right\rangle+h(x)\right\} \\
& -\frac{1}{\tau}\left\{\lambda c(x) u_{1}+\left\langle M(x) D u_{1}, D u_{1}\right\rangle+h(x)\right\} \\
& \geq \lambda c(x) w+\langle M(x) D w, D w\rangle+h(x) \quad \text { in } \Omega,
\end{aligned}
$$

since $F$ is convex in $(r, p, X)$ and $p \mapsto\langle M(x) p, p\rangle$ is convex in $p$. Now, by remark 6.24 we have $w \ll u_{0}$ in $\Omega$, i.e. $w=w_{\tau}<0$ in $\Omega$. Then, there exists a little bit smaller $t \in(0, \tau)$ such that $w_{t}<0$ in $\Omega$ (see the argument in proposition 4.4, by taking a compact set with small measure containing the boundary). Therefore, this last contradicts the definition of $\tau$ as a minimum. 


\section{5}

\section{Proof of Theorem 5.5}

Suppose, for the time being, just $u_{0} \geq 0$ with $c u_{0} \supsetneqq 0$ in $\Omega$ and (SC) 0 .

Claim 6.26 We have $u \gg u_{0}$, for every nonnegative $L^{p}$-viscosity supersolution $u \in E$ of $\left(P_{\lambda}\right)$, for all $\lambda>0$.

Proof. Notice that $\lambda c(x) u \geq 0$ in $\Omega$ implies that $u$ is an $L^{p}$-viscosity supersolution of $\left(P_{0}\right)$. Since $u_{0}$ is strong, by remark $5.9, u \geq u_{0}$ in $\Omega$. But $u_{0}$ is not a solution of $\left(P_{\lambda}\right)$ for $\lambda>0$ since $c u_{0} \not \equiv 0$, which means that $u_{0} \not \equiv u$.

Set $v:=u-u_{0}$ in $\Omega$. Then, using $M \geq 0$, we see that $v$ is a nonnegative $L^{p}$-viscosity supersolution of

$$
\mathcal{M}^{-}\left(D^{2} v\right)-\widetilde{b}(x)|D v| \leq 0 \quad \text { in } \Omega
$$

with $\widetilde{b}:=b+2 \mu_{2}\left|D u_{0}\right|$, as usual. By SMP, $v>0$ in $\Omega$. If $v>0$ on $\partial \Omega$ it is done; if on the other side there exists $x_{0} \in \partial \Omega$ with $v\left(x_{0}\right)=0$, we apply Hopf lemma to obtain $\partial_{\nu} v\left(x_{0}\right)>0$. Therefore, $v \gg 0$ in $\Omega$.

Claim 6.27 $\left(P_{\lambda}\right)$ has no nonnegative $L^{p}$-viscosity solutions for $\lambda$ large.

Proof. Let $\lambda \geq \widetilde{\lambda}_{1}$, where $\widetilde{\lambda}_{1}=\widetilde{\lambda}_{1}^{+}\left(\widetilde{\mathcal{L}}^{-}(c), \Omega\right)>0$ is the principal weighted eigenvalue of

$$
\widetilde{\mathcal{L}}^{-}[v]:=\mathcal{M}^{-}\left(D^{2} v\right)+\widetilde{b}(x)|D v|, \quad \widetilde{b}(x):=b(x)+2 \mu_{2}\left|D u_{0}\right| \in L_{+}^{p}(\Omega),
$$

associated to $\widetilde{\varphi}_{1}=\widetilde{\varphi}_{1}^{+}\left(\widetilde{\mathcal{L}}^{-}(c), \Omega\right) \in W^{2, p}(\Omega)$, from proposition 4.3 , i.e.

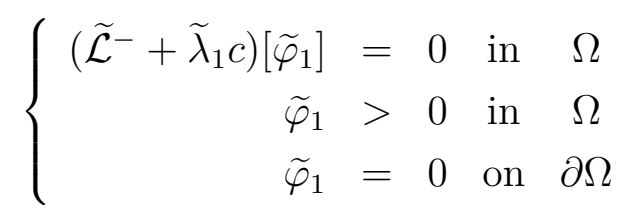

Suppose, in order to obtain a contradiction, that there exists a nonnegative $L^{p}$-viscosity solution $u$ of $\left(P_{\lambda}\right)$ and set $v:=u-u_{0}$ in $\Omega$. By claim 6.26 , it follows that $v \gg 0$ in $\Omega$.

Since $u_{0}$ is strong, we can use it as a test function into the definition of $L^{p}$-viscosity supersolution of $u$, together with $(\mathrm{SC})_{0}$ and $\mu_{2} I \geq M \geq 0$, to obtain that

$$
\begin{aligned}
-\mathcal{L}^{-}[v] & \geq \lambda c(x) v+\lambda c(x) u_{0}+\langle M(x) D v, D v\rangle+\left\langle M(x) D v, D u_{0}\right\rangle \\
& +\left\langle M(x) D u_{0}, D v\right\rangle \supsetneqq \widetilde{\lambda}_{1} c(x) v-2 \mu_{2}\left|D u_{0}\right||D v|
\end{aligned}
$$


since $c(x) u_{0} \supsetneqq 0$, and so $v$ satisfies

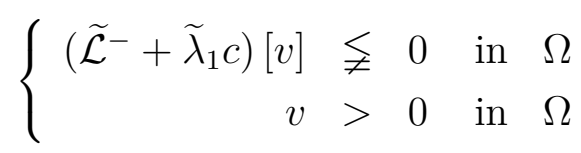

in the $L^{p}$-viscosity sense. As the proof of lemma 6.8, applying proposition 4.4 (and remark 4.6) to (6.26) and (6.25), we get $v=t \widetilde{\varphi}_{1}$ for $t>0$. But this contradicts the first line in $(6.26)$, since $\left(\widetilde{\mathcal{L}}^{-}+\widetilde{\lambda}_{1} c\right)\left[t \widetilde{\varphi}_{1}\right]=0$ in $\Omega$.

Define

$$
\bar{\lambda}:=\sup \left\{\lambda ;\left(P_{\lambda}\right) \text { has an } L^{p} \text {-viscosity solution } u_{\lambda} \geq 0 \text { in } \Omega\right\}
$$

which is finite, by claim 6.27. Of course it is well defined and nonnegative, since $u_{0} \geq 0$. Also, by the definition of $\bar{\lambda},\left(P_{\lambda}\right)$ has no nonnegative solutions for $\lambda>\bar{\lambda}$.

It is a subtle but important detail that $\bar{\lambda}$ is a positive number. In fact, by the existence of the continuum (theorem 5.3) we know that, for $\lambda$ small, there exists a solution $u_{\lambda}$ of $\left(P_{\lambda}\right)$ such that $u_{\lambda} \not \equiv 0$, since $\left\|u_{0}\right\|_{E}>0$. But why can we infer that $u_{\lambda} \geq 0$ for small $\lambda$ positive? This is the subject of the next claim.

Consider $\left(H_{2}\right)$ from now. Then, $L^{p}$-viscosity solutions of $\left(P_{\lambda}\right)$ are strong.

Claim 6.28 $\bar{\lambda}>0$.

Proof. Let $\lambda \in\left(0, \Lambda_{0}\right)$, where $\Lambda_{0}$ is such that there exists a nontrivial solution of $\left(P_{\lambda}\right)$ in this interval, as indicated above. Suppose $\Lambda_{0} \leq \min \left\{1,1 / C_{0}\right\}$, where $C_{0}$ is a lower bound for the solutions of $\left(P_{\lambda}\right)$ such that $u \geq-C_{0}$, for all $\lambda \in[0,1]$. We are supposing here $C_{0}>0$, otherwise every solution of $\left(P_{\lambda}\right)$ would be nonnegative for $\lambda \leq 1$.

Suppose firstly that $h \geq c$. In this case every nontrivial solution $u$ of $\left(P_{\lambda}\right)$ satisfies

$$
\left\{\begin{aligned}
-\mathcal{L}^{-}[u] & \geq-F[u] \geq c(x)\left(1-\Lambda_{0} C_{0}\right)+\langle M(x) D u, D u\rangle \geq 0 & & \text { in } \Omega \\
u & =0 & & \text { on } \partial \Omega .
\end{aligned}\right.
$$

Then $u \geq 0$ in $\Omega$ by ABP, for all $\lambda \in\left(0, \Lambda_{0}\right)$.

In the general case, let $u_{c}$ a nontrivial nonnegative solution of the problem

$$
\left\{\begin{aligned}
-F\left[u_{c}\right] & =\widetilde{h}(x)+\lambda c(x) u_{c}+\left\langle M(x) D u_{c}, D u_{c}\right\rangle \geq 0 & & \text { in } \quad \Omega \\
u_{c} & =0 & & \text { on } \quad \partial \Omega
\end{aligned}\right.
$$

for $\widetilde{h}:=\max \{h, c\} \geq c$. Notice that $u_{c}$ is strong under $\left(H_{2}\right)$, since $|\widetilde{h}| \leq|h|+c$. Moreover, since $\widetilde{h} \geq h$, then $u_{c}$ is a supersolution of $\left(P_{\lambda}\right)$. Therefore $u_{c} \geq u_{0}$ 
by claim 6.26. Further, since $\lambda c(x) u_{0} \geq 0, u_{0}$ is a strong subsolution of $\left(P_{\lambda}\right)$. Thus, applying theorem 6.3 , we obtain an $L^{p}$-viscosity solution $u$ of $\left(P_{\lambda}\right)$ with $u_{0} \leq u \leq u_{c}$ in $\Omega$. In particular this solution is nonnegative and nontrivial.

Remark 6.29 Another way to prove claim 6.5 is through arguments on first eigenvalues, which provide an estimate on the smallness of $\lambda$.

Set $v:=u_{0}-u$, with $u$ an $L^{p}$-viscosity solution of $\left(P_{\lambda}\right)$ for positive $\lambda$ such that $\lambda<\left(C_{A}\|c\|_{L^{p}(\Omega)}\right)^{-1}$, where $C_{A}$ is the constant from $A B P$ for $\mu=0$. Of course, negativity of $v$ yields $u \geq u_{0} \geq 0$ in $\Omega$. So, in order to obtain a contradiction, suppose that $\sup _{\Omega} v>0$.

Notice that $v$ is an $L^{p}$-viscosity solution of $\widetilde{\mathcal{L}}^{+}[v] \geq-\lambda c(x) v^{+}$in $\Omega$, with $v=0$ on $\partial \Omega$. Thus, as in the proof of proposition 3.4 in [12], we use ABP to obtain that $\sup _{\Omega} v \leq \lambda C_{A}\|c\|_{L^{p}(\Omega)} \sup _{\Omega} v^{+}$which, by the choice of $\lambda$, yields a contradiction.

Claim 6.30 For each $\lambda \in(0, \bar{\lambda}),\left(P_{\lambda}\right)$ has a well ordered strict pair of strong sub and supersolutions, namely $u_{0} \ll \beta_{\lambda}$ in $\Omega$.

Proof. Let $\lambda \in(0, \bar{\lambda})$. As the strict subsolution we just consider $u_{0}$ again, which is strong. Note that $u_{0}$ is strict, since for any supersolution $u \in E$ such that $u \geq u_{0}$ in $\Omega$, we have $u \gg u_{0}$, by repeating the final paragraph in the proof of claim 6.26 .

Note that, from the definition of $\bar{\lambda}$, there exists $\mu \in(\lambda, \bar{\lambda})$ and a nonnegative solution $u_{\mu}$ of $\left(P_{\mu}\right)$. By claim 6.26, $u_{\mu} \gg u_{0}$ in $\Omega$. On the other hand, since

$$
c(x)(\mu-\lambda) u_{\mu} \geq c(x)(\mu-\lambda) u_{0} \supsetneqq 0,
$$

we have $\mu c(x) u_{\mu} \supsetneqq \lambda c(x) u_{\mu}$ and so $u_{\mu}$ is a supersolution of $\left(P_{\lambda}\right)$ which is not a solution. In addition, $u_{\mu}$ is strict because if $u \in E$ is an $L^{p}$-viscosity subsolution of $\left(P_{\lambda}\right)$ with $u \leq u_{\mu}$ in $\Omega$, by defining $v=u_{\mu}-u$ and arguing as usual when we have a strong supersolution, $v$ becomes an $L^{p}$-viscosity supersolution of

$$
\mathcal{M}^{-}\left(D^{2} v\right)-\widetilde{b}(x)|D v|-\mu_{2}|D v|^{2} \supsetneqq 0 \quad \text { in } \Omega
$$

with $\widetilde{b}:=b+2 \mu_{2}\left|D u_{\mu}\right| \in L_{+}^{p}(\Omega)$. Thus, $w=\frac{1}{m}\left\{1-e^{-m v}\right\}$, for $m=\mu_{2} / \lambda_{P}$, is an $L^{p}$-viscosity supersolution of $\mathcal{M}^{-}\left(D^{2} w\right)-\widetilde{b}(x)|D w| \supsetneqq 0$ in $\Omega$ by lemma 2.20 . Then, SMP gives us $w>0$ in $\Omega$ and so $w \gg 0$ in $\Omega$, by applying Hopf at the boundary bounds where $w=0$. Consequently, $v \gg 0$ in $\Omega$.

Therefore, we can define $\beta_{\lambda}:=u_{\mu}$ for $\mu=\mu(\lambda)$, for all $\lambda \in(0, \bar{\lambda})$.

Hence, by claim 6.30 and theorem 6.3 , there exists a solution $u_{\lambda, 1}$ of $\left(P_{\lambda}\right)$ with $u_{0} \leq u_{\lambda, 1} \leq \beta_{\lambda}$ in $\Omega$ and $\operatorname{deg}\left(I-\mathcal{T}_{\lambda}, \mathcal{S}, 0\right)=1$, for all $\lambda>0$. 
Next, we work a little bit more to construct the second solution $u_{\lambda, 2}$ that also satisfies $u_{\lambda, 2} \gg u_{0}$ but is not in $\mathcal{S}$ (as in [15]). For this, fix a $\lambda \in(0, \bar{\lambda})$ and consider the open subset of $E$ defined by

$$
\mathcal{D}=\left\{u \in C_{0}^{1}(\bar{\Omega}) ; u_{0} \ll u \text { in } \Omega\right\}
$$

which contains the set $\mathcal{S}$ from theorem 6.3 , since

$$
\mathcal{S}=\left\{u \in \mathcal{B}_{R} ; u \ll \beta_{\lambda} \text { in } \Omega\right\} \cap \mathcal{D} .
$$

Analogously to the proof of claim 6.16 , we obtain an a priori $L^{\infty}$ bound for the solutions of $\left(P_{\lambda, k}\right)$ which depends on $\lambda$ but not on $k \in[0,1]$, related to $\Lambda_{2}:=\bar{\lambda}-\delta$ for some small $\delta>0$. This provides a $R_{0}=R_{0}(\lambda, \delta)>R$ which bounds the $E$-norm of the solutions, by the $C^{1, \alpha}$ estimates. Then, by the homotopy invariance of the degree in $k$ and the fact that there is no solution for $k=1$, we have $\operatorname{deg}\left(I-\mathcal{T}_{\lambda}, \mathcal{B}_{R_{0}} \cap \mathcal{D}, 0\right)=0$. Therefore, by excision, $\operatorname{deg}\left(I-\mathcal{T}_{\lambda},\left(\mathcal{B}_{R_{0}} \cap \mathcal{D}\right) \backslash \mathcal{S}, 0\right)=-1$, which provides a second solution $u_{\lambda, 2} \in \mathcal{D} \backslash \mathcal{S}$, i.e. a solution that satisfies, by construction, $u_{\lambda, 2} \gg u_{0}$ in $\Omega$, for $\lambda \in(0, \bar{\lambda}-\delta]$, for every $\delta>0$. In particular, this second solution is also nonnegative and nontrivial for all $\lambda<\bar{\lambda}$.

Under $\left(H_{2}\right)$, theorem $6.3(i i)$ allows us to choose $u_{\lambda, 1}$ as the minimal strong solution between $u_{0}$ and $\beta_{\lambda}$. As the proof of claim 6.19, this implies (6.21). Indeed, $u_{\lambda, 1} \neq u_{\lambda, 2}$ and, if would exist $x_{0} \in \Omega$ with $u_{\lambda, 1}\left(x_{0}\right)>$ $u_{\lambda, 2}\left(x_{0}\right)$, by defining $\tilde{\beta}=\tilde{\beta}_{\lambda}:=\min \left\{u_{\lambda, 1}, u_{\lambda, 2}, \beta_{\lambda}\right\}$, as the minimum of strong supersolutions greater or equal than $u_{0}$, we have $\tilde{\beta} \geq u_{0}$ in $\Omega$. Also, $\tilde{\beta} \leq \beta_{\lambda}$ in $\Omega$. By theorem 6.3 there exists a solution $u$ of $\left(P_{\lambda}\right)$ such that $u_{0} \leq u \leq \tilde{\beta} \supsetneqq u_{\lambda, 1}$, which contradicts the minimality of $u_{\lambda, 1}$, since $u$ is a solution which belongs to the order interval $\left[u_{0}, \beta_{\lambda}\right]$.

Therefore, defining $v=u_{\lambda, 2}-u_{\lambda, 1} \supsetneqq 0$ in $\Omega$, we see that $v$ is a nonnegative strong solution of $\mathcal{M}^{-}\left(D^{2} v\right)-\widetilde{b}(x)|D v| \leq 0$ in $\Omega$. Thus, since $v \not \equiv 0$, SMP yields $v>0$ in $\Omega$ and Hopf concludes that $v \gg 0$ in $\Omega$, i.e.

$$
u_{\lambda, 1} \ll u_{\lambda, 2} \text { in } \Omega \text {, for all } \lambda \in(0, \bar{\lambda}) \text {. }
$$

Claim 6.31 For $\lambda_{1}<\lambda_{2}$, we have $u_{\lambda_{1}, 1} \ll u_{\lambda_{2}, 1}$ in $\Omega$.

Proof. The proof is similar to the proof of claim 6.30, but a little bit simpler since both $u_{\lambda_{1}, 1}$ and $u_{\lambda_{2}, 2}$ are strong. We repeat it here to avoid confusions about notation. For fixed $\lambda_{1}<\lambda_{2}$, we have $\lambda_{2} c(x) u_{\lambda_{2}, 1} \supsetneqq \lambda_{1} c(x) u_{\lambda_{2}, 1}$ since

$$
\left(\lambda_{2}-\lambda_{1}\right) c(x) u_{\lambda_{2}, 1} \geq\left(\lambda_{2}-\lambda_{1}\right) c(x) u_{0} \supsetneqq 0 .
$$


Then, $u_{\lambda_{2}, 1}$ is a supersolution of $\left(P_{\lambda_{1}}\right)$ that is not a solution. In particular it follows that $u_{\lambda_{1}, 1} \neq u_{\lambda_{2}, 1}$.

Next $u_{\lambda_{1}, 1} \supsetneqq u_{\lambda_{2}, 1}$ in $\Omega$. In fact, if there was a point $x_{0} \in \Omega$ such that $u_{\lambda_{1}, 1}\left(x_{0}\right)>u_{\lambda_{2}, 1}\left(x_{0}\right)$, by defining $\tilde{\beta}=\min \left\{u_{\lambda_{1}, 1}, u_{\lambda_{2}, 1}, \beta_{\lambda_{1}}\right\}$, the minimum of strong supersolutions of $\left(P_{\lambda_{1}}\right)$ larger than $u_{0}$, we would have that $u_{0} \leq \tilde{\beta}$ in $\Omega$. By theorem 6.3, there exists a solution $u$ of $\left(P_{\lambda_{2}}\right)$ such that $u_{0} \leq u \leq \tilde{\beta} \supsetneqq u_{\lambda_{1}, 1}$, which contradicts the minimality of $u_{\lambda_{1}, 1}$, since $u$ is a solution that belongs to the order interval $\left[u_{0}, \beta_{\lambda_{1}}\right]$.

Hence the function $v:=u_{\lambda_{2}, 1}-u_{\lambda_{1}, 1} \supsetneqq 0$ is a strong supersolution of $\mathcal{M}^{-}\left(D^{2} v\right)-\widetilde{b}(x)|D v| \leq 0$ in $\Omega$, for $\widetilde{b}=b+2 \mu_{2}\left|D u_{\lambda_{1}, 1}\right|$. Then SMP yields $v>0$ in $\Omega$, since $v \not \equiv 0$. Next, Hopf and $v=0$ on $\partial \Omega$ give us $\left.\partial_{\nu} v\right|_{\partial \Omega}>0$, from where $v \gg 0$ in $\Omega$.

The existence proof of at least one solution for $\left(P_{\bar{\lambda}}\right)$ follows exactly the same lines as the proof of claim 6.18 since, in there, we only used the fact that there exists one sequence of solutions corresponding to a maximizing sequence of $\lambda$ 's converging to the supremum $\bar{\lambda}$. Furthermore, uniqueness is true if $F$ is convex in $(r, p, X)$, by following the proof of claim 6.20. Finally, the behavior of the solutions is the same as in claim 6.17 and this finishes the proof of theorem 5.5.

Remark 6.32 Particular cases of theorems 5.4 and 5.5 are $h \supsetneqq 0$ and $h \supsetneqq 0$, respectively, when the hypothesis $\left(H_{0}\right)$ holds. Indeed, if $h \supsetneqq 0$, then $u_{0}$ is a strong supersolution of

$$
-\mathcal{L}^{-}\left[u_{0}\right] \geq-F\left[u_{0}\right]=h(x)+\left\langle M(x) D u_{0}, D u_{0}\right\rangle \supsetneqq 0 \quad \text { in } \Omega
$$

with $u_{0}=0$ on $\partial \Omega$. Then SPM gives us $u_{0}>0$ in $\Omega$ and so $c u_{0} \not \equiv 0$. Furthermore, by Hopf, $u_{0} \gg 0$ in $\Omega$.

On the other hand, if $h \supsetneqq 0$, then $u_{0}$ is a strong subsolution of

$$
-\mathcal{L}^{+}\left[u_{0}\right] \leq-F\left[u_{0}\right] \supsetneqq\left\langle M(x) D u_{0}, D u_{0}\right\rangle \quad \text { in } \Omega
$$

and so $v_{0}:=\frac{1}{m}\left(e^{m u_{0}}-1\right)$, for $m=\frac{\mu_{2}}{\lambda_{P}}$, is a strong subsolution of $\mathcal{L}^{+}\left[v_{0}\right] \supsetneqq 0$ in $\Omega$ by lemma 2.20, with $v_{0}=0$ on $\partial \Omega$. Again by $S M P$ we get $v_{0}<0$ in $\Omega$ (then $v_{0} \ll 0$ in $\Omega$ by Hopf ) and so does $u_{0}$ (with $\left.u_{0} \ll 0\right)$, from where $c u_{0} \not \equiv 0$.

Notice that in the case $h \equiv 0$, we have that $u \equiv 0$ is a strong solution of $\left(P_{\lambda}\right)$, for all $\lambda \in \mathbb{R}$. By theorem 1(iii) in [12], this is the unique $L^{p}$-viscosity solution for all $\lambda \leq 0$. By theorem 5.3 we obtain the existence of $\bar{\lambda}$ such that $\left(P_{\lambda}\right)$ has at least one more nontrivial solution, for all $\lambda \in(0, \bar{\lambda})$. 


\section{Bibliography}

[1] CRANDALL, M. G. Condition d'unicité pour les solutions généralisées des équations de Hamilton-Jacobi du premier ordre, C. R. Acad. Sci. Paris Sér. I Math., v.292, n.3, p. 183-186, 1981.

[2] CRANDALL, M. G.; LIONS, P.-L. Viscosity solutions of Hamilton-Jacobi equations, Transactions of the American Mathematical Society, v.277, n.1, p. 1-42, 1983.

[3] CRANDALL, M. G.; ISHII, H. ; LIONS, P.-L. User's guide to viscosity solutions of second order partial differential equations, Bulletin of the American Mathematical Society, v.27, n.1, p. 1-67, 1992.

[4] CAFFARELli, L.; CRANDALL, M. G.; KOCAN, M. ; ŚWIĘCH, A. On viscosity solutions of fully nonlinear equations with measurable ingredients, Communications on Pure and Applied Mathematics, v.49, n.4, p. 365-398, 1996.

[5] NADIRASHVILI, N. Nonuniqueness in the martingale problem and the Dirichlet problem for uniformly elliptic operators, Annali della Scuola Normale Superiore di Pisa-Classe di Scienze, v.24, n.3, p. 537-549, 1997.

[6] BOCCARDO, L.; MURAT, F. ; PUEL, J. P. Existence de solutions faibles pour des équations elliptiques quasi-linéaires à croissance quadratique. In: Nonlinear partial differential equations and their applications, Collège de France Seminar, vol. 4, volume 4, p. 19-73. Research Notes in Mathematics, vol. 84, Pitman London, 1983.

[7] BOCCARDO, L.; MURAT, F. ; PUEL, J. P. Résultats d'existence pour certains problèmes elliptiques quasilinéaires, Annali Della Scuola Normale Superiore Di Pisa-Classe Di Scienze, v.11, n.2, p. 213-235, 1984.

[8] KAZDAN, J. L.; KRAMER, R. J. Invariant criteria for existence of solutions to second-order quasilinear elliptic equations, Communications on Pure and Applied Mathematics, v.31, n.5, p. 619-645, 1978. 
[9] BARleS, G.; BLANC, A.-P.; GEORGELIN, C. ; KOBYLANSKI, M. Remarks on the maximum principle for nonlinear elliptic pdes with quadratic growth conditions, Annali della Scuola Normale Superiore di Pisa. Classe di scienze, v.28, n.3, p. 381-404, 1999.

[10] BARLES, G.; MURAT, F. Uniqueness and the maximum principle for quasilinear elliptic equations with quadratic growth conditions, Archive for rational mechanics and analysis, v.133, n.1, p. 77-101, 1995.

[11] FERONE, V.; MURAT, F. Nonlinear problems having natural growth in the gradient: an existence result when the source terms are small, Nonlinear Analysis: Theory, Methods and Applications, v.42, n.7, p. $1309-1326,2000$.

[12] SIRAKOV, B. Solvability of uniformly elliptic fully nonlinear pde, Archive for Rational Mechanics and Analysis, v.195, n.2, p. 579-607, 2010.

[13] ARCOYA, D.; DE COSTER, C.; JEANJEAN, L. ; TANAKA, K. Continuum of solutions for an elliptic problem with critical growth in the gradient, Journal of Functional Analysis, v.268, n.8, p. 2298-2335, 2015.

[14] DE COSTER, C.; FERNÁNDEZ, A. J. Existence and multiplicity for elliptic p-Laplacian problems with critical growth in the gradient, arXiv:1801.04155, 2018.

[15] DE COSTER, C.; JEANJEAN, L. Multiplicity results in the non-coercive case for an elliptic problem with critical growth in the gradient, Journal of Differential Equations, v.262, n.10, p. 5231-5270, 2017.

[16] JEANJEAN, L.; RAMOS QUOIRIN, H. Multiple solutions for an indefinite elliptic problem with critical growth in the gradient, Proceedings of the American Mathematical Society, v.144, n.2, p. 575-586, 2016.

[17] JEANJEAN, L.; SIRAKOV, B. Existence and multiplicity for elliptic problems with quadratic growth in the gradient, Communications in Partial Differential Equations, v.38, n.2, p. 244-264, 2013.

[18] SOUPLET, P. A priori estimates and bifurcation of solutions for an elliptic equation with semidefinite critical growth in the gradient, Nonlinear Analysis: Theory, Methods and Applications, v.121, p. 412-423, 2015. 
[19] NORNBERG, G.; SIRAKOV, B. A priori bounds and multiplicity for fully nonlinear equations with quadratic growth in the gradient, Preprint, arXiv:1802.01661, 2018.

[20] NORNBERG, G. S. $C^{1, \alpha}$ regularity for fully nonlinear elliptic equations with superlinear growth in the gradient, arXiv:1802.01643, 2018.

[21] FLEMING, W. H.; SONER, H. M. Controlled Markov processes and viscosity solutions, volume 25 of Stochastic Modelling and Applied Probability. Second. ed., Springer, New York, 2006. 429p.

[22] GOMES, D. A.; NURBEKYAN, L. ; PIMENTEL, E. A. Economic models and mean-field games theory. Publicações Matemáticas do IMPA. [IMPA Mathematical Publications]. Instituto Nacional de Matemática Pura e Aplicada (IMPA), Rio de Janeiro, 2015. 127p. 30o Colóquio Brasileiro de Matemática. [30th Brazilian Mathematics Colloquium].

[23] SIRAKOV, B. Boundary Harnack estimates and quantitative strong maximum principles for uniformly elliptic pde, International Mathematics Research Notices, p. 1-26, 2017.

[24] KOIKE, S.; ŚWIĘCH, A. Existence of strong solutions of pucci extremal equations with superlinear growth in $D u$, Journal of Fixed Point Theory and Applications, v.5, n.2, p. 291-304, 2009.

[25] KOIKE, S.; ŚWIECCH, A. Weak Harnack inequality for fully nonlinear uniformly elliptic pde with unbounded ingredients, Journal of the Mathematical Society of Japan, v.61, n.3, p. 723-755, 2009.

[26] CAFFARELLI, L. A.; CABRÉ, X. Fully nonlinear elliptic equations, volume 43. Colloquium Publications, 43. American Mathematical Society, Providence, RI, 1995. 104p.

[27] SIRAKOV, B. Modern theory of nonlinear elliptic PDE. 30th Colóquio Brasileiro de Matemática, 2015.

[28] QUAAS, A.; SIRAKOV, B. Principal eigenvalues and the Dirichlet problem for fully nonlinear elliptic operators, Advances in Mathematics, v.218, n.1, p. 105-135, 2008.

[29] EVANS, L. C. Partial differential equations, volume 19. American Mathematical Society, 2nd ed, 2010. 749p.

[30] BREZIS, H. Functional analysis, Sobolev spaces and partial differential equations. Springer Science \& Business Media, 2011. 599p. 
[31] AMBROSIO, L.; DANCER, N. Calculus of variations and partial differential equations. Springer-Verlag, Berlin, 2000. 347p. Topics on geometrical evolution problems and degree theory, Papers from the Summer School held in Pisa, September 1996, Edited by G. Buttazzo, A. Marino and M. K. V. Murthy.

[32] HAN, Q.; LIN, F. Elliptic partial differential equations. Courant Institute of Mathematical Sciences, volume 1. American Mathematical Society, 1997. 123p.

[33] KOIKE, S. A beginners guide to the theory of viscosity solutions. 2nd ed, 2010.

[34] CRANDAlL, M. G.; KOCAN, M.; SORAVIA, P. ; ŚWIEcCH, A. On the equivalence of various weak notions of solutions of elliptic pdes with measurable ingredients. In: Progress in elliptic and parabolic partial differential equations, p. 136--162. Citeseer, 1996.

[35] KOIKE, S. Perron's method : revisited (viscosity solution theory of differential equations and its developments), Departmental Bulletin Paper, v.1428, n.4, p. 1-8, 2005.

[36] KOIKE, S.; ŚWIEcCH, A. Maximum principle for fully nonlinear equations via the iterated comparison function method, Mathematische Annalen, v.339, n.2, p. 461-484, 2007.

[37] NAKAGAWA, K. Maximum principle for $L^{p}$-viscosity solutions of fully nonlinear equations with unbounded ingredients and superlinear growth terms, Advances in Mathematical Sciences and Applications, v.19, n.1, p. 89-107, 2009.

[38] WINTER, N. $W^{2, p}$ and $W^{1, p}$-estimates at the boundary for solutions of fully nonlinear, uniformly elliptic equations, Zeitschrift für Analysis und ihre Anwendungen, v.28, n.2, p. 129-164, 2009.

[39] BARDI, M.; DA LIO, F. On the strong maximum principle for fully nonlinear degenerate elliptic equations, Archiv der Mathematik, v.73, n.4, p. 276-285, 1999.

[40] BRAGA, J. E. M.; MOREIRA, D. Inhomogeneous Hopf-Oleinik lemma and regularity of semiconvex supersolutions via new barriers for the Pucci extremal operators, to appear in Advances in Mathematics. 
[41] GILBARG, D.; TRUDINGER, N. S. Elliptic partial differential equations of second order. Springer, 2nd ed, 2001. 529p.

[42] KOIKE, S.; ŚWIECH, A. Local maximum principle for $L^{p}$-viscosity solutions of fully nonlinear pdes with unbounded ingredients, Communications on Pure \& Applied Analysis, v.11, n.5, p. 1897-1910, 2012.

[43] WANG, L. On the regularity theory of fully nonlinear parabolic equations: II, Communications on pure and applied mathematics, v.45, n.2, p. 141-178, 1992.

[44] ISHII, H.; LIONS, P.-L. Viscosity solutions of fully nonlinear second-order elliptic partial differential equations, Journal of Differential equations, v.83, n.1, p. 26-78, 1990.

[45] ISHII, H. On uniqueness and existence of viscosity solutions of fully nonlinear second-order elliptic pde's, Communications on pure and applied mathematics, v.42, n.1, p. 15-45, 1989.

[46] MILAKIS, E.; SILVESTRE, L. E. Regularity for fully nonlinear elliptic equations with Neumann boundary data, Communications in Partial Differential Equations, v.31, n.8, p. 1227-1252, 2006.

[47] CAFFARELLI, L. A. Interior a priori estimates for solutions of fully non-linear equations, Annals of Mathematics, v.130, n.1, p. 189-213, 1989.

[48] ŚWIECH, A. $\quad W^{1, p}$-interior estimates for solutions of fully nonlinear, uniformly elliptic equations, Advances in Differential Equations, v.2, n.6, p. 1005-1027, 1997.

[49] TRUDINGER, N. S. On regularity and existence of viscosity solutions of nonlinear second order, elliptic equations. In: Partial differential equations and the calculus of variations, p. 939-957. Birkhauser, Boston, 1989.

[50] TRUDINGER, N. S. Hölder gradient estimates for fully nonlinear elliptic equations, Proceedings of the Royal Society of Edinburgh Section A: Mathematics, v.108, n.1-2, p. 57-65, 1988.

[51] SILVESTRE, L.; SIRAKOV, B. Boundary regularity for viscosity solutions of fully nonlinear elliptic equations, Communications in Partial Differential Equations, v.39, n.9, p. 1694-1717, 2014. 
[52] LADYZHENSKAYA, O. A.; URAL'TSEVA, N. N. Linear and quasilinear equations of elliptic type, 1968.

[53] KRYLOV, N. V. Nonlinear elliptic and parabolic equations of the second order, volume 7. Springer, 1987.

[54] LADYZHENSKAYA, O. A.; URAL'TSEVA, N. N. Estimates on the boundary of the domain of first derivatives of functions satisfying an elliptic or a parabolic inequality. boundary value problems of mathematical physics. part 13, work collection, Trudy Matematicheskogo Instituta imeni VA Steklova, v.179, p. 102-125, 1988.

[55] SILVESTRE, L.; TEIXEIRA, E. V. Regularity estimates for fully non linear elliptic equations which are asymptotically convex. In: Contributions to Nonlinear Elliptic Equations and Systems, p. 425-438. Progr. Nonlinear Differential Equations Appl., 86, Birkhäuser/Springer, Cham, 2015.

[56] IMBERT, C.; SILVESTRE, L. $\quad C^{1, \alpha}$ regularity of solutions of some degenerate fully non-linear elliptic equations, Advances in Mathematics, v.233, n.1, p. 196-206, 2013.

[57] BIRINDELLI, I.; DEMENGEL, F. $\quad C^{1, \beta}$ regularity for Dirichlet problems associated to fully nonlinear degenerate elliptic equations, ESAIM: Control, Optimisation and Calculus of Variations, v.20, n.4, p. 1009-1024, 2014.

[58] CRANDALL, M. G.; KOCAN, M. ; ŚWIĘCH, A. $L^{p}$-theory for fully nonlinear uniformly parabolic equations: Parabolic equations, Communications in Partial Differential Equations, v.25, n.11-12, p. 1997-2053, 2000.

[59] KRYLOV, N. V. On $C^{1+\alpha}$ regularity of solutions of Isaacs parabolic equations with vmo coefficients, Nonlinear Differential Equations and Applications NoDEA, v.21, n.1, p. 63-85, 2014.

[60] WANG, L. On the regularity theory of fully nonlinear parabolic equations: I, Communications on pure and applied mathematics, v.45, n.1, p. 27-76, 1992.

[61] DA SILVA, J. V.; TEIXEIRA, E. V. Sharp regularity estimates for second order fully nonlinear parabolic equations, Mathematische Annalen, v.369, n.3-4, p. 1623-1648, 2017. 
[62] BRAGA, J. E. M.; MOREIRA ; WANG, L. Inhomogeneous Hopf-Oleinik lemma and applications. part iv: Sharp Krylov boundary gradient type estimates for solutions to fully nonlinear differential inequalities with unbounded coefficients and $C^{1 \text {,Dini }}$ boundary data, arXiv:1608.02352, 2016.

[63] KOIKE, S.; ŚWIECCH, A. Maximum principle and existence of $L^{p}$-viscosity solutions for fully nonlinear uniformly elliptic equations with measurable and quadratic terms, Nonlinear Differential Equations and Applications NoDEA, v.11, n.4, p. 491-509, 2004.

[64] ARMSTRONG, S. N. Principal eigenvalues and an anti-maximum principle for homogeneous fully nonlinear elliptic equations, J. Differential Equations, v.246, n.7, p. 2958-2987, 2009.

[65] TROIANIELLO, G. M. Elliptic differential equations and obstacle problems. Springer Science \& Business Media, New York, 1987.

[66] BANDLE, C.; REICHEL, W. Solutions of quasilinear second-order elliptic boundary value problems via degree theory, volume 1. Elsevier, NorthHolland, Amsterdam, 2004. 70p.

[67] QUAAS, A. Existence of a positive solution to a "semilinear" equation involving pucci's operator in a convex domain, Differential and Integral Equations, v.17, n.5-6, p. 481-494, 2004.

[68] HESS, P.; KATO, T. On some linear and nonlinear eigenvalue problems with an indefinite weight function, Communications in Partial Differential Equations, v.5, n.10, p. 999-1030, 1980.

[69] ISHII, H.; YOSHIMURA, Y. Demi-eigenvalues for uniformly elliptic Isaacs operators, preprint, 2005.

[70] BERESTYCKI, H.; NIRENBERG, L. ; VARADHAN, S. R. S. The principal eigenvalue and maximum principle for second-order elliptic operators in general domains, Communications on Pure and Applied Mathematics, v.47, n.1, p. 47-92, 1994.

[71] GARCÍA-MELIÁN, J.; ROSSI, J. D. On the principal eigenvalue of some nonlocal diffusion problems, Journal of Differential Equations, v.246, n.1, p. 21-38, 2009.

[72] BIRINDELLI, I.; DEMENGEL, F. Eigenvalue, maximum principle and regularity for fully non linear homogeneous operators, Commun. Pure Appl. Anal., v.6, n.2, p. 335-366, 2007. 
[73] BIRINDELLI, I.; DEMENGEL, F. Regularity and uniqueness of the first eigenfunction for singular fully nonlinear operators, Journal of Differential Equations, v.249, n.5, p. 1089-1110, 2010.

[74] LIONS, P.-L. Bifurcation and optimal stochastic control, Nonlinear Analysis: Theory, Methods and Applications, v.7, n.2, p. 177-207, 1983.

[75] Berestycki, H.; DOLCETTA, I. C.; PORRETTA, A. ; ROSSI, L. Maximum principle and generalized principal eigenvalue for degenerate elliptic operators, Journal de Mathématiques Pures et Appliquées, v.103, n.5, p. 1276-1293, 2015.

[76] IKOMA, N.; ISHII, H. Eigenvalue problem for fully nonlinear second-order elliptic pde on balls. In: Annales de I'Institut Henri Poincare Anal. Non Linéaire, volume 29, p. 783-812. Elsevier, 2012.

[77] IKOMA, N.; ISHII, H. Eigenvalue problem for fully nonlinear second-order elliptic pde on balls, ii, Bulletin of Mathematical Sciences, v.5, n.3, p. 451-510, 2015.

[78] PATRIZI, S. Principal eigenvalues for Isaacs operators with Neumann boundary conditions, Nonlinear Differential Equations and Applications NoDEA, v.16, n.1, p. 79-107, 2009.

[79] GIDAS, B.; SPRUCK, J. A priori bounds for positive solutions of nonlinear elliptic equations, Communications in Partial Differential Equations, v.6, n.8, p. 883-901, 1981.

[80] CHANG, K. C. Methods in nonlinear analysis. Springer Monographs in Mathematics, 2005. 442p.

[81] AMBrosetTI, A.; MALCHIODI, A. Nonlinear analysis and semilinear elliptic problems, volume 104 of Cambridge Studies in Advanced Mathematics. Cambridge University Press, Cambridge, 2007. $316 p$.

[82] ARCOYA, D.; DE COSTER, C.; JEANJEAN, L. ; TANAKA, K. Remarks on the uniqueness for quasilinear elliptic equations with quadratic growth conditions, Journal of Mathematical Analysis and Applications, v.420, n.1, p. 772-780, 2014.

[83] ABDELlAOUI, B.; PERAL, I. ; PRIMO, A. Elliptic problems with a hardy potential and critical growth in the gradient: Non-resonance and blow-up results, Journal of Differential Equations, v.239, n.2, p. 386-416, 2007. 
[84] ABDEllaOUI, B.; DALL'AGLiO, A. ; PERAL, I. Some remarks on elliptic problems with critical growth in the gradient, Journal of Differential Equations, v.222, n.1, p. 21-62, 2006.

[85] PIMENTEL, E. A.; TEIXEIRA, E. V. Sharp Hessian integrability estimates for nonlinear elliptic equations: an asymptotic approach, Journal de Mathématiques Pures et Appliquées, v.106, n.4, p. 744-767, 2016.

[86] SIRAKOV, B. Uniform bounds via regularity estimates for elliptic pde with critical growth in the gradient, arXiv:1509.04495, 2015.

[87] VÁZQUEZ, J. L. A strong maximum principle for some quasilinear elliptic equations, Applied Mathematics and Optimization, v.12, n.1, p. 191-202, 1984.

[88] MUNKRES, J. R. Topology. 2nd ed. Prentice Hall, Upper Saddle River, NJ 07458, 2000. 537p.

[89] BUSCA, J.; SIRAKOV, B. Harnack type estimates for nonlinear elliptic systems and applications. In: Annales de l'Institut Henri Poincare (C) Non Linear Analysis, volume 21, p. 543-590. Elsevier, 2004.

[90] FOK, P.-K. Some maximum principles and continuity estimates for fully nonlinear elliptic equations of second order, Ph.D. Thesis, University of California at Santa Barbara, 1996.

[91] TRUDINGER, N. S. Local estimates for subsolutions and supersolutions of general second order elliptic quasilinear equations, Inventiones mathematicae, v.61, n.1, p. 67-79, 1980. 
A

\section{Notations}

Here we list some notations used throughout the text.

- $\mathbb{S}^{n}$ is the set of $n \times n$ symmetric matrices with real entries, $\mathbb{S}^{n} \subset \mathbb{M}_{n \times n}(\mathbb{R})$;

- $B_{r}\left(x_{0}\right)=\left\{x \in \mathbb{R}^{n} ;\left|x-x_{0}\right|<r\right\}$ is a ball with radius $r$ in $\mathbb{R}^{n}$ centered at $x_{0}$, for $r>0, B_{r}=B_{r}(0)$;

- $B_{r}^{+}=B_{r} \cap\left\{x_{n}>0\right\}, \mathbb{T}_{r}=B_{r} \cap\left\{x_{n}=0\right\}$, for $r>0$;

- $B_{r}^{\nu}\left(x_{0}\right)=B_{r}\left(x_{0}\right) \cap\left\{x_{n}>-\nu\right\}, \mathbb{T}_{r}^{\nu}\left(x_{0}\right)=B_{r}\left(x_{0}\right) \cap\left\{x_{n}=-\nu\right\}, r, \nu>0$;

- $\omega$ is a Lipschitz modulus if $\omega(r) \leq \omega(1) r$ for all $r \geq 0$;

- $\Omega$ is a bounded domain (open and connected) in $\mathbb{R}^{n} ; u: \Omega \rightarrow \mathbb{R}$;

- $\partial_{\nu}$ is the derivative in the direction of the interior unit normal;

- We say $f \in L_{+}^{p}(\Omega)$ to mean a real function $f: \Omega \rightarrow \mathbb{R}$ such that $f \in L^{p}(\Omega)$ and $f \geq 0$;

- $D u=\left(u_{x_{1}}, \ldots, u_{x_{n}}\right)$ is the gradient of $u$;

- $D^{2} u=\left(\partial_{i j} u\right)_{i, j=1}^{n}$ is the Hessian matrix of $u$, where $\partial_{i j} u=\frac{\partial^{2} u}{\partial x_{i} \partial x_{j}}$;

- $\Delta u=\sum_{i=1}^{n} \partial_{i i} u=\operatorname{tr}\left(D^{2} u\right)$ is the Laplacian of $u$;

- $u \in U S C(\Omega)$ means that $\overline{\lim }_{y \rightarrow x} u(y)=u(x)$ for all $x \in \Omega$, and it is equivalent to have $\{x \in \Omega ; u(x)<\alpha\}$ open for all $\alpha \in \mathbb{R}$;

- $\mathcal{L}^{ \pm}[u]=\mathcal{M}^{ \pm}\left(D^{2} u\right) \pm b(x)|D u|$ denote both $\mathcal{L}^{+}[u]=\mathcal{M}^{+}\left(D^{2} u\right)+b(x)|D u|$ and $\mathcal{L}^{-}[u]=\mathcal{M}^{-}\left(D^{2} u\right)-b(x)|D u|$

- In chapters 5 and $6, E=C^{1}(\bar{\Omega})$ and $\mathcal{B}_{r}=\mathcal{B}_{r}^{E}(0)=\left\{u \in E ;\|u\|_{E}<r\right\}$ is the ball in $E$, for any $r>0$. 


\section{B}

\section{Quadratic ABP}

In this chapter we give a simple proof of quadratic ABP in the case $p>n$, with $\mu$ a positive constant. The proof is a version of theorem 3.1 in [63] (for constant b). For more general conditions see also theorems 2.6 in [25] and 3.4 in [37] (see also theorem 3.3 of [24]), even for unbounded $\mu$.

Proof of Proposition 2.6. We prove the result for subsolutions, since in the supersolution case we just need to apply the former result to $-u$. We can suppose $0 \in \Omega$ and consider $B=B_{2 d}(0)$ for $d=\operatorname{diam}(\Omega)$. We define $g: B \rightarrow \mathbb{R}$,

$$
g:=f^{-}+d^{-\frac{n}{p}}\left\|f^{-}\right\|_{L^{p}(\Omega)} \text { in } \Omega, \quad g:=0 \text { in } B \backslash \Omega
$$

then $\|g\|_{L^{p}(B)} \leq\left\|f^{-}\right\|_{L^{p}(\Omega)}+d^{-\frac{n}{p}}\left\|f^{-}\right\|_{L^{p}(\Omega)}|\Omega|^{\frac{1}{p}} \leq\left(1+\left|B_{1}\right|^{\frac{1}{p}}\right)\left\|f^{-}\right\|_{L^{p}(\Omega)}$. Also, by Holder's inequality, $\|g\|_{L^{n}(B)} \leq|B|^{\frac{1}{n}-\frac{1}{p}}\|g\|_{L^{p}(B)} \leq d^{1-\frac{n}{p}} C_{n, p}\left\|f^{-}\right\|_{L^{p}(\Omega)}$.

By theorem $3.5^{1}$, there exists a unique strong solution $w \in W^{2, p}(B)$ of

$$
\mathcal{M}_{\lambda, \Lambda}^{+}\left(D^{2} w\right)+b(x)|D w|=-g(x) \text { in } B
$$

with $w=0$ on $\partial B$, for $b \in L_{+}^{p}(B)$ defined as zero in $B \backslash \Omega$, with

$$
\|w\|_{L^{\infty}(B)} \leq C_{A}\|g\|_{L^{n}(B)} \leq d^{1-\frac{n}{p}} C_{n, p} C_{A}\left\|f^{-}\right\|_{L^{p}(\Omega)},
$$

where $C_{A}=d C_{1}$ is the constant from $\mathrm{ABP}$ in the $L^{n}$ case, and also

$$
\|w\|_{W^{2, p}\left(B^{\prime}\right)} \leq C\left\{\|w\|_{L^{\infty}(B)}+\|g\|_{L^{p}(B)}\right\} \leq C_{2}\left\|f^{-}\right\|_{L^{p}(\Omega)}
$$

for all $B^{\prime} \subset \subset B$, where $C_{2}=C_{2}\left(n, p, \lambda, \Lambda,\|b\|_{L^{p}(\Omega)}, d\right.$, $\left.\operatorname{dist}\left(B^{\prime}, \partial B\right)\right)$. By the Sobolev embedding $W^{2, p} \subset W^{1, \infty}$, the last inequality and the $L^{p}$ estimate for $g$, we obtain, for $B^{\prime}:=B_{d}(0) \subset \subset B=B_{2 d}(0)$,

$$
\|D w\|_{L^{\infty}(\Omega)} \leq\|D w\|_{L^{\infty}\left(B^{\prime}\right)} \leq C_{n}\|w\|_{W^{2, p}\left(B^{\prime}\right)} \leq C_{0}\left\|f^{-}\right\|_{L^{p}(\Omega)}
$$

where $C_{0}=C_{n} C_{2}=C_{0}\left(n, p, \lambda, \Lambda,\|b\|_{L^{p}(\Omega)}, d\right)$.

${ }^{1}$ Of course, we use that $C^{1, \alpha}$ regularity and theorem 3.5, for unbounded coefficients in the case $\mu=0$, are already available. After, we come back here and show quadratic ABP for $\mu \neq 0$, which is needed to prove $C^{1, \alpha}$ regularity for $\mu \neq 0$. 
We first claim that if $\mu\left\|f^{-}\right\|_{L^{p}(\Omega)} d^{\frac{n}{p}} \leq \delta=: 1 / C_{0}^{2}$, then the function $v:=u-w-\max _{\partial \Omega} u$ is an $L^{p}$-viscosity subsolution of

$$
\mathcal{M}_{\lambda, \Lambda}^{+}\left(D^{2} v\right)+\widetilde{b}(x)|D v|+\mu|D v|^{2} \geq 0 \text { in } \Omega, \quad v \leq 0 \text { on } \partial \Omega,
$$

where $\widetilde{b}(x)=b(x)+2 \mu\|D w\|_{L^{\infty}(\Omega)} \in L^{p}(\Omega)$. To prove this, let $\phi \in W_{\text {loc }}^{2, p}(\Omega)$. Since $w$ is a strong solution, we can write that

$$
\begin{aligned}
\mathcal{M}_{\lambda, \Lambda}^{+}( & \left.D^{2}(w+\phi)\right)+b(x)|D(w+\phi)|+\mu|D(w+\phi)|^{2} \\
\leq & \left\{\mathcal{M}_{\lambda, \Lambda}^{+}\left(D^{2} w\right)+b(x)|D w|\right\}+\mathcal{M}_{\lambda, \Lambda}^{+}\left(D^{2} \phi\right) \\
& \quad+b(x)|D \phi|+2 \mu|D w||D \phi|+\mu|D \phi|^{2}+\mu|D w|^{2} \\
\leq- & g(x)+\left\{\mathcal{M}_{\lambda, \Lambda}^{+}\left(D^{2} \phi\right)+\widetilde{b}(x)|D \phi|+\mu|D \phi|^{2}\right\}+\mu \mid\|D w\|_{L^{\infty}(\Omega)}^{2} \\
\leq- & f^{-}(x)-d^{-\frac{n}{p}}\left\|f^{-}\right\|_{L^{p}(\Omega)}+\mu C_{0}^{2}\left\|f^{-}\right\|_{L^{p}(\Omega)}^{2} \\
& \quad+\left\{\mathcal{M}_{\lambda, \Lambda}^{+}\left(D^{2} \phi\right)+\widetilde{b}(x)|D \phi|+\mu|D \phi|^{2}\right\} \\
\leq & f(x)+\left\{\mathcal{M}_{\lambda, \Lambda}^{+}\left(D^{2} \phi\right)+\widetilde{b}(x)|D \phi|+\mu|D \phi|^{2}\right\} \text { for a.e. } x \in \Omega
\end{aligned}
$$

by the choice $\mu C_{0}^{2}\left\|f^{-}\right\|_{L^{p}(\Omega)} d^{\frac{n}{p}} \leq 1$. So, if $v-\phi$ attains a maximum at $x_{0} \in \Omega$, then $u-(w+\phi)$ attains a maximum at $x_{0} \in \Omega$, where $w+\varphi \in W_{\text {loc }}^{2, p}(\Omega)$. Hence, by the definition of $u$ being an $L^{p}$-viscosity subsolution,

$$
\begin{aligned}
0 & \leq \text { ess } \varlimsup_{x \rightarrow x_{0}}\left\{\mathcal{M}_{\lambda, \Lambda}^{+}\left(D^{2}(w+\phi)\right)+b(x)|D(w+\phi)|+\mu|D(w+\phi)|^{2}-f(x)\right\} \\
& \leq \text { ess } \varlimsup_{x \rightarrow x_{0}}\left\{\mathcal{M}_{\lambda, \Lambda}^{+}\left(D^{2} \phi\right)+\widetilde{b}(x)|D \phi|+\mu|D \phi|^{2}\right\} .
\end{aligned}
$$

Of course, $v \leq 0$ on $\partial \Omega$ by construction and the claim is established.

Next, $v \leq 0$ in $\Omega$. Indeed, $V:=\frac{1}{m}\left(e^{m v}-1\right)$ is an $L^{p}$-viscosity solution of $\mathcal{M}_{\lambda, \Lambda}^{+}\left(D^{2} V\right)+\widetilde{b}(x)|D V| \geq 0$ in $\Omega$ by lemma 2.20 , where $m=\mu / \lambda$. Further, $V \leq 0$ on $\partial \Omega$. Then by ABP we have $V \leq 0$ in $\Omega$, as well as for $v$. Therefore,

$$
u-\max _{\partial \Omega} u \leq w \leq\|w\|_{L^{\infty}(\Omega)} \leq C_{B}\left\|f^{-}\right\|_{L^{p}(\Omega)} \text { in } \Omega,
$$

where $C_{B}:=d^{2-\frac{n}{p}} C_{n, p} C_{1}$ depends on $n, p, \lambda, \Lambda, \operatorname{diam}(\Omega),\|b\|_{L^{p}(\Omega)}$ and it remains bounded if these quantities are bounded.

See the foregoing works [25], [37], [24] for a quadratic version of ABP for $p_{0}<p \leq n$ (which is known as GMP); their proofs are based on the iterative method. 


\section{C}

\section{A property of functions}

To prove $C^{1, \alpha}$ estimates, the usual way is to show that some function $u$, solution of a given equation in the $L^{p}$-viscosity sense, satisfies

$$
|u(x)-u(y)-D u(y) \cdot(x-y)| \leq C|x-y|^{1+\alpha}, \quad|D u(y)| \leq C
$$

for all $x, y$ in a suitable domain. How this implies that $D u$ belongs to $C^{\alpha}$ and

$$
|D u(x)-D u(y)| \leq C|x-y|^{\alpha},
$$

is a well known universal fact which does not depend on the equation, it is only a property of functions. A direct proof for it can be found in [27]. We reproduce it below for completeness, adding some improvements done by Prof. Boyan Sirakov. We also refer to the appendix of [62] for an elementary proof.

In the one dimensional case $n=1,($ C.2) is evident from (C.1), since for each $x, y \in B_{1 / 2}=(-1 / 2,1 / 2), x>y$, we have

$$
\left\{\begin{array}{l}
\max \left\{u(x)-u(y)-u^{\prime}(y)(x-y),-u(x)+u(y)+u^{\prime}(y)(x-y)\right\} \leq C|x-y|^{\alpha+1} \\
\max \left\{u(y)-u(x)-u^{\prime}(x)(y-x),-u(y)+u(x)+u^{\prime}(x)(y-x)\right\} \leq C|x-y|^{\alpha+1}
\end{array}\right.
$$

and thus, by summing, $\left|u^{\prime}(x)-u^{\prime}(y)\right|(x-y) \leq C|x-y|^{\alpha+1}$.

In the general case we argue by induction on $n$. Suppose (C.2) valid for each function of $n-1$ variables which satisfies (C.1) and let $u$ be a differentiable function of $n$ variables satisfying (C.1) for each $x, y \in B_{1 / 2}$. Fix $\bar{x}, \bar{y} \in B_{1 / 2}$. We want to show that

$$
|D u(\bar{x})-D u(\bar{y})| \leq C|\bar{x}-\bar{y}|^{\alpha},
$$

where $C$ depends on the right quantities. Without loss we can assume that $\bar{x}, \bar{y}$ belong to the plane $\left\{x_{n}=0\right\}$ (otherwise just rotate the space). Set

$$
v=\left.u\right|_{x_{n}=0}:\left(\mathbb{R}^{n-1} \approx \mathbb{R}^{n} \cap\left\{x_{n}=0\right\}\right) \cap B_{1} \rightarrow \mathbb{R}
$$


so the induction hypothesis holds for $v$ and gives us

$$
\left|D_{x^{\prime}} u(x)-D_{x^{\prime}} u(y)\right| \leq C|x-y|^{\alpha} \text {, for all } x, y \in\left\{x_{n}=0\right\} \cap B_{1 / 2} .
$$

where $x=\left(x^{\prime}, x_{n}\right)$. Thus we only need to show that

$$
\left|\partial_{x_{n}} u(\bar{x})-\partial_{x_{n}} u(\bar{y})\right| \leq C|\bar{x}-\bar{y}|^{\alpha} .
$$

We can assume $|\bar{x}-\bar{y}|<1 / 4$ (otherwise we are done since the gradient of $u$ is bounded). Set $r=2|\bar{x}-\bar{y}|$ and fix some point $z \in B_{r}(\bar{x}) \cap B_{r}(\bar{y}) \cap B_{1 / 2}$ such that $z_{n}=r / 4$. Then

$$
\begin{aligned}
& \left|\partial_{x_{n}} u(\bar{x})-\partial_{x_{n}} u(\bar{y})\right|=\frac{4}{r}\left|\partial_{x_{n}} u(\bar{x}) \cdot z_{n}-\partial_{x_{n}} u(\bar{y}) \cdot z_{n}\right| \\
& =\frac{4}{r} \mid u(z)-u(\bar{y})-D_{x^{\prime}} u(\bar{y}) \cdot\left(z^{\prime}-\bar{y}^{\prime}\right)-\partial_{x_{n}} u(\bar{y}) \cdot z_{n} \\
& -\left\{u(z)-u(\bar{y})-D_{x^{\prime}} u(\bar{y}) \cdot\left(z^{\prime}-\bar{y}^{\prime}\right)\right\} \\
& -\left\{u(z)-u(\bar{x})-D_{x^{\prime}} u(\bar{x}) \cdot\left(z^{\prime}-\bar{x}^{\prime}\right)-\partial_{x_{n}} u(\bar{x}) \cdot z_{n}\right\} \\
& +u(z)-u(\bar{x})-D_{x^{\prime}} u(\bar{x}) \cdot\left(z^{\prime}-\bar{x}^{\prime}\right) \mid \\
& =\frac{4}{r} \mid u(z)-u(\bar{y})-D_{x} u(\bar{y}) \cdot(z-\bar{y})-\left\{u(z)-u(\bar{x})-D_{x} u(\bar{x}) \cdot(z-\bar{x})\right\} \\
& +u(\bar{y})-u(\bar{x})+D_{x^{\prime}} u(\bar{y}) \cdot\left(z^{\prime}-\bar{y}^{\prime}\right)-D_{x^{\prime}} u(\bar{x}) \cdot\left(z^{\prime}-\bar{x}^{\prime}\right) \mid \\
& \leq \frac{4}{r}\left\{\left|u(z)-u(\bar{y})-D_{x} u(\bar{y}) \cdot(z-\bar{y})\right|+\left|u(z)-u(\bar{x})-D_{x} u(\bar{x}) \cdot(z-\bar{x})\right|\right. \\
& \left.+\left|u(\bar{y})-u(\bar{x})-D_{x^{\prime}} u(\bar{x}) \cdot\left(\bar{y}^{\prime}-\bar{x}^{\prime}\right)\right|+\left|\left(D_{x^{\prime}} u(\bar{y})-D_{x^{\prime}} u(\bar{x})\right) \cdot\left(z^{\prime}-\bar{y}^{\prime}\right)\right|\right\}
\end{aligned}
$$

where we subtracted and added $D_{x^{\prime}} u(\bar{x}) \cdot\left(z^{\prime}-\bar{y}^{\prime}\right)$ to get the last expression. Now we estimate each one of the above terms separately. By using (C.1),

$$
\begin{gathered}
\left|u(z)-u(\bar{y})-D_{x} u(\bar{y}) \cdot(z-\bar{y})\right| \leq C|z-\bar{y}|^{\alpha+1} \leq C r^{\alpha+1}=C 2^{\alpha+1}|\bar{x}-\bar{y}|^{\alpha+1} \\
\left|u(z)-u(\bar{x})-D_{x} u(\bar{x}) \cdot(z-\bar{x})\right| \leq C|z-\bar{x}|^{\alpha+1} \leq C r^{\alpha+1}=C 2^{\alpha+1}|\bar{x}-\bar{y}|^{\alpha+1} \\
\left|u(\bar{y})-u(\bar{x})-D_{x^{\prime}} u(\bar{x}) \cdot\left(\bar{y}^{\prime}-\bar{x}^{\prime}\right)\right| \leq C|\bar{x}-\bar{y}|^{\alpha+1}
\end{gathered}
$$

since $D_{x^{\prime}} u(\bar{x}) \cdot\left(\bar{y}^{\prime}-\bar{x}^{\prime}\right)=D_{x} u(\bar{x}) \cdot(\bar{y}-\bar{x})$. Finally, we conclude by using, in the last modulus, the induction hypothesis

$$
\left|D_{x^{\prime}} u(\bar{y})-D_{x^{\prime}} u(\bar{x})\right|\left|z^{\prime}-\bar{y}^{\prime}\right| \leq C|\bar{x}-\bar{y}|^{\alpha} r=2 C|\bar{x}-\bar{y}|^{\alpha+1} .
$$

Consequently, from (C.2), we derive the estimate

$$
\|u\|_{1, \alpha}=\|u\|_{\infty}+\|D u\|_{\infty}+[D u]_{\alpha} \leq(1+2 C) W
$$

with $C$ replaced by $C W$ (which is the appropriate constant), $W$ being equal to $\|u\|_{\infty}$ plus another terms, depending on each case, local or global one. 
D

\section{The Local Maximum Principle for $C$-viscosity solutions}

We present a detailed proof of theorem 2.2 in the case $b$ is bounded and $\nu$ is continuous in $B_{3}$, following the ideas of [89], [90], [41], [33] and [91]. Consider here $\mathcal{L}^{ \pm}[u]:=\mathcal{M}_{\lambda, \Lambda}^{ \pm}\left(D^{2} u\right) \pm \gamma|D u|, \quad \gamma \geq 0$.

Prof of Theorem 2.2. Let $1 \leq r<p$, for the time being. The conclusion is trivial if $u \equiv 0$ in $B_{2}$, thus suppose $u \not \equiv 0$. So $v:=u^{+} \supsetneqq 0$ in $B_{2}$.

Case 1. $f \in C\left(B_{3}\right)$ : In this case consider the sup-convolution of $v$ as

$$
v^{\varepsilon}:=\sup _{y \in B_{5 / 2}}\left\{v(y)-\frac{|x-y|^{2}}{2 \varepsilon}\right\} .
$$

Then, as in lemma 6.1 in [89], we know that $v^{\varepsilon} \in C^{0,1}\left(B_{5 / 2}\right)$, it is twice differentiable a.e. in $B_{2}$ and $v^{\varepsilon} \rightarrow v$ uniformly in $\bar{B}$, for every $B \subset \subset B_{5 / 2}$. Moreover, from (2.6), $v^{\varepsilon}$ satisfies, in the $C$-viscosity sense (and also a.e.),

$$
\mathcal{L}^{+}\left[v^{\varepsilon}\right]+\nu(x) v_{\varepsilon} \geq-f_{\varepsilon}(x) \text { in } B_{5 / 2}^{\varepsilon}=\left\{x \in B_{5 / 2} ; \operatorname{dist}(x, \partial \Omega)>\delta_{\varepsilon}\right\}
$$

(and in particular in $B_{2}$ for $\left.\varepsilon \leq \varepsilon_{0}\right)$, for $\delta_{\varepsilon}=2\left(\varepsilon\|v\|_{L^{\infty}\left(B_{5 / 2}\right)}\right)^{1 / 2}$ and

$$
v_{\varepsilon}(x):=\sup _{|x-y|<\delta_{\varepsilon}} v^{\varepsilon}(y), \quad f_{\varepsilon}(x):=\sup _{|x-y|<\delta_{\varepsilon}} f^{+}(y) .
$$

Set $w_{\varepsilon}:=\eta v^{\varepsilon}$ in $B_{2-\delta_{\varepsilon}}$, where $\eta \in[0,1]$ is a cut-off function defined as

$$
\eta(x)=\eta_{\varepsilon}(x):=\frac{\left(\left(2-\delta_{\varepsilon}\right)^{2}-|x|^{2}\right)^{\beta}}{\left(2-\delta_{\varepsilon}\right)^{2 \beta}}
$$

for some $\beta \geq 2$ to be chosen. Notice that, for $\delta=\delta_{\varepsilon}, D \eta(x)=-\frac{2 \beta x}{(2-\delta)^{2}} \eta^{1-\frac{1}{\beta}}$ and $D^{2} \eta(x)=\frac{2 \beta}{(2-\delta)^{4}}\left\{2(\beta-1) x \otimes x-\left((2-\delta)^{2}-|x|^{2}\right) I\right\} \eta^{1-\frac{2}{\beta}}$.

Thus it holds a.e. that

$$
\begin{aligned}
- & \mathcal{L}^{+}\left[w_{\varepsilon}\right]=\mathcal{M}^{-}\left(-\eta D^{2} v^{\varepsilon}-2 D \eta \otimes D v^{\varepsilon}-v^{\varepsilon} D^{2} \eta\right)-\gamma\left|\eta D v^{\varepsilon}+v^{\varepsilon} D \eta\right| \\
& \leq \mathcal{M}^{-}\left(-\eta D^{2} v^{\varepsilon}\right)+2 \mathcal{M}^{+}\left((-D \eta) \otimes D v^{\varepsilon}\right)+\mathcal{M}^{+}\left(-v^{\varepsilon} D^{2} \eta\right) \\
& -\eta \gamma\left|D v^{\varepsilon}\right|+\gamma\left|v^{\varepsilon} D \eta\right| \\
& \leq-\eta \mathcal{L}^{+}\left[v^{\varepsilon}\right]+2 \Lambda|D \eta|\left|D v^{\varepsilon}\right|+\gamma\left|v^{\varepsilon}\right||D \eta| \\
& +\frac{2 \beta}{(2-\delta)^{4}}\left\{2(\beta-1) \mathcal{M}^{+}\left(-v^{\varepsilon} x \otimes x\right)+\left((2-\delta)^{2}-|x|^{2}\right) \mathcal{M}^{+}\left(v^{\varepsilon} I\right)\right\} \eta^{1-\frac{2}{\beta}}
\end{aligned}
$$




$$
\begin{aligned}
& \leq \eta\left\{\nu(x) v_{\varepsilon}+f_{\varepsilon}(x)\right\}+\frac{4 \beta \Lambda}{(2-\delta)^{2}}|x|\left|D v^{\varepsilon}\right| \eta^{1-\frac{1}{\beta}}+\frac{2 \beta}{(2-\delta)^{2}} \gamma|x|\left|v^{\varepsilon}\right| \eta^{1-\frac{1}{\beta}} \\
& +\frac{2 \beta \Lambda}{(2-\delta)^{4}}\left|v^{\varepsilon}\right|\left\{2(\beta-1)|x|^{2}+n\left((2-\delta)^{2}-|x|^{2}\right)\right\} \eta^{1-\frac{2}{\beta}}
\end{aligned}
$$

since the only nonzero eigenvalue of $x \otimes x$ is $|x|^{2}$. Since $v^{\varepsilon}$ is Lipschitz in $B_{5 / 2}$, it belongs to $W^{1, \infty}(B)[29$, p. 294]. So $u$ is differentiable a.e., its derivative coincides with the weak derivative a.e. [29, p. 295] and $D v^{\varepsilon} \in L^{\infty}\left(B_{5 / 2}\right)$. Thus, naming by $H$ the right hand side of the above, we have that $H \in L^{p}\left(B_{2-\delta}\right)$, since

$$
\left\|\eta v_{\varepsilon} \nu\right\|_{L^{p}\left(B_{2-\delta}\right)} \leq\|\nu\|_{L^{p_{1}\left(B_{2}\right)}}\left\|\eta v_{\varepsilon}\right\|_{L^{q_{1}\left(B_{2-\delta}\right)}}, \quad \text { where } \frac{1}{p}=\frac{1}{p_{1}}+\frac{1}{q_{1}}
$$

i.e. for $q_{1}=\frac{p p_{1}}{p_{1}-p}>p \geq n$, Holder's inequality and Young yield

$$
\left\|\eta v_{\varepsilon}\right\|_{L^{q_{1}\left(B_{2-\delta}\right)}} \leq \theta\left\|\eta v_{\varepsilon}\right\|_{L^{q_{2}\left(B_{2-\delta}\right)}}+\theta^{-\mu}\left\|\eta v_{\varepsilon}\right\|_{L^{r}\left(B_{2-\delta}\right)}, \mu=\frac{1 / r-1 / q_{1}}{1 / q_{1}-1 / q_{2}}
$$

where $1 \leq r \leq q_{1} \leq q_{2}$ and $\theta>0$.

Remark D.1 (Contact sets in $A B P$ in the case $\mu=0$ ) If $b \in L_{+}^{\infty}(\Omega)$ or if $u \in W_{\text {loc }}^{2, p}(\Omega)$, the $L^{p}$ norm of the function $f$ can be taken over $\Gamma_{u}^{+} \cap \Omega^{+}$ (respectively $\Gamma_{u}^{-} \cap \Omega^{-}$), the upper (lower) contact set of the function $u$, where

$$
\Gamma_{u}^{+}\left(\Gamma_{u}^{-}\right):=\left\{x \in \Omega ; \exists p \in \mathbb{R}^{n} ; u(y) \leq(\geq) u(x)+p \cdot(y-x), \forall y \in \Omega\right\} .
$$

See the proofs of proposition 6.2 in [33] and theorem 9.1 in [41].

Now we use lemma 2.8 in [4] (see also lemma 7.10 in [33]) to obtain that $w_{\varepsilon}$ is also an $L^{p}$-viscosity solution of (D.2). Then, by ABP and remark D.1,

$$
\sup _{B_{2-\delta}} w_{\varepsilon} \leq C_{A}(2-\delta)^{2-\frac{n}{p}}\|H\|_{L^{p}\left(\Gamma_{\varepsilon}^{+} \cap\left\{w_{\varepsilon}>0\right\}\right)} .
$$

Claim D.2 $w_{\varepsilon}(x) \geq\left|D w_{\varepsilon}(x)\right|(2-\delta-|x|)$, a.e. $x \in \Gamma_{\varepsilon}^{+}$.

Proof. Fix a point $x \in \Gamma_{\varepsilon}^{+}$of differentiability of $w=w_{\varepsilon}$ with $|D w(x)|>0$ (otherwise it is obvious). Notice that $|x|=2-\delta-m_{x}, m_{x} \in(0,2-\delta]$, so take $k_{0}=k_{0}(x) \in \mathbb{N}$ such that $m_{x}>\frac{1}{k_{0}} \geq \frac{1}{k}$, for all $k \geq k_{0}$. Next choose

$$
y_{k}=y_{k}(x):=x-t_{k} \frac{D w(x)}{|D w(x)|}, \quad \text { for } t_{k}=2-\delta-|x|-\frac{1}{k} .
$$

Then $y_{k} \in B_{2-\delta}(0)$, since $\left|y_{k}\right|-|x| \leq\left|y_{k}-x\right|=2-\delta-|x|-\frac{1}{k} \Rightarrow\left|y_{k}\right|<2-\delta$, and so $0 \leq w\left(y_{k}\right) \leq w(x)+D w(x) \cdot\left(y_{k}-x\right)=w(x)-t_{k}|D w(x)|$ for all $k \geq k_{0}$, which proves claim D.2 by passing to limits as $k \rightarrow+\infty$. 
Then, using claim D.2 and definition of $\eta$, we have for a.e. $x \in \Gamma_{\varepsilon}^{+}$,

$$
\begin{aligned}
\left|D v^{\varepsilon}\right| & \leq \frac{1}{\eta}\left\{\left|D w_{\varepsilon}\right|+v^{\varepsilon}|D \eta|\right\} \leq \frac{1}{\eta}\left\{\frac{w_{\varepsilon}}{2-\delta+|x|}+v^{\varepsilon} \frac{2 \beta|x|}{(2-\delta)^{2}} \eta^{1-\frac{1}{\beta}}\right\} \\
& \leq v^{\varepsilon} \eta^{-\frac{1}{\beta}}\left\{\frac{2(2-\delta)}{(2-\delta)^{2 / \beta}}+\frac{2 \beta(2-\delta)}{(2-\delta)^{2}}\right\} \leq 4(1+\beta) v^{\varepsilon} \eta^{-\frac{1}{\beta}}
\end{aligned}
$$

with $\varepsilon \leq \varepsilon_{0}=\varepsilon\left(\|v\|_{\infty}\right)$ chosen such that $\delta=\delta_{\varepsilon} \leq 1$. Thus

$$
\begin{aligned}
|H| \leq & \eta \nu(x) v_{\varepsilon}+\eta f_{\varepsilon}(x)+\frac{16 \beta \Lambda}{2-\delta}(1+\beta) v^{\varepsilon} \eta^{1-\frac{2}{\beta}}+\frac{2 \beta \gamma}{2-\delta} v^{\varepsilon} \eta^{1-\frac{1}{\beta}} \\
& +\frac{4 \beta \Lambda}{(2-\delta)^{2}}(\beta-1+n) v^{\varepsilon} \eta^{1-\frac{2}{\beta}} \\
\leq & \eta \nu(x) v_{\varepsilon}+\eta f_{\varepsilon}(x)+\{16 \beta \Lambda(1+\beta)+2 \beta \gamma+4 \beta \Lambda(\beta-1+n)\} w_{\varepsilon} \eta^{-\frac{2}{\beta}} \\
\leq & \eta \nu(x) v_{\varepsilon}+f_{\varepsilon}(x)+C w_{\varepsilon}^{1-\frac{2}{\beta}}\left(w_{\varepsilon} / \eta\right)^{\frac{2}{\beta}}
\end{aligned}
$$

where $C=C(n, \beta, \gamma, \Lambda)$. Hence, for $L^{p}=L^{p}\left(B_{2-\delta}\right)$,

$$
\|H\|_{L^{p}\left(\Gamma_{\varepsilon}^{+}\right)} \leq\left\|\eta \nu(x) v_{\varepsilon}\right\|_{L^{p}}+\left\|f_{\varepsilon}\right\|_{L^{p}}+C\left(\sup _{\Gamma_{\varepsilon}^{+}} w_{\varepsilon}\right)^{1-\frac{2}{\beta}}\left\|\left(v^{\varepsilon}\right)^{\frac{2}{\beta}}\right\|_{L^{p}} .
$$

Therefore, by the latter, (D.3) and (D.4) applied to (D.5), we obtain

$$
\begin{aligned}
\sup _{B_{2-\delta}} w_{\varepsilon} \leq & C_{0} \theta\|\nu\|_{L^{p_{1}\left(B_{2}\right)}}\left\|\eta v_{\varepsilon}\right\|_{L^{q_{2}\left(B_{2-\delta}\right)}}+C_{0} \theta^{-\mu}\|\nu\|_{L^{p_{1}\left(B_{2}\right)}}\left\|\eta v_{\varepsilon}\right\|_{L^{r}\left(B_{2}\right)} \\
& +C_{0}\left\|f_{\varepsilon}\right\|_{L^{p}\left(B_{3}\right)}+c_{0}\left(\sup _{\Gamma_{\varepsilon}^{+}} w_{\varepsilon}\right)^{1-\frac{2}{\beta}}\left(\int_{B_{2}}\left(v^{\varepsilon}\right)^{r}\right)^{1 / p}
\end{aligned}
$$

for $C_{0}=2^{1-\frac{n}{p}} C_{A}$, by choosing $\beta=2 p / r \geq 2$, since $1 \leq r \leq p$.

We are going to use Young's inequality in the form

$$
a b \leq \sigma a^{p_{0}}+\sigma^{-\frac{q_{0}}{p_{0}}} b^{q_{0}}, \text { for } p_{0}, q_{0} \in(1,+\infty) \text { such that } \frac{1}{p_{0}}+\frac{1}{q_{0}}=1 .
$$

By taking $a=\left(\sup _{\Gamma_{\varepsilon}^{+}} w_{\varepsilon}\right)^{1-\frac{2}{\beta}}, b=1, p_{0}=1 /\left(1-\frac{2}{\beta}\right)=\frac{p}{p-r}>1$, so $q_{0}=\frac{p}{r}>1$, and choosing $\sigma=\frac{1}{2 c_{0}}\left(\int_{B_{2}}\left(v^{\varepsilon}\right)^{r}\right)^{-1 / p}$, we have

$$
c_{0}\left(\sup _{\Gamma_{\varepsilon}^{+}} w_{\varepsilon}\right)^{1-\frac{2}{\beta}}\left(\int_{B_{2}}\left(v^{\varepsilon}\right)^{r}\right)^{1 / p} \leq \frac{1}{2} \sup _{B_{2-\delta}} w_{\varepsilon}+c\left(\int_{B_{2}}\left(v^{\varepsilon}\right)^{r}\right)^{1 / r} .
$$

Note that if $\|\nu\|_{L^{p_{1}\left(B_{2}\right)}}=0$, the first two addends on the right hand side of (D.6) disappear. Therefore, in (D.6) we assume $\|\nu\|_{L^{p_{1}\left(B_{2}\right)}}>0$ and set $\theta=\left(4 C_{0}\left|B_{2}\right|^{1 / q_{2}}\|\nu\|_{L^{p_{1}\left(B_{2}\right)}}\right)^{-1} ;$ thus

$$
\sup _{B_{2}-\delta} w_{\varepsilon} \leq \frac{1}{2} \sup _{B_{2-\delta}}\left(\eta v_{\varepsilon}\right)+c\|\nu\|_{L^{p_{1}}}^{\mu+1}\left\|\eta v_{\varepsilon}\right\|_{L^{r}}+2 C_{0}\left\|f_{\varepsilon}\right\|_{L^{p}}+2 c\left(\int_{B_{2}}\left(v^{\varepsilon}\right)^{r}\right)^{1 / r}
$$


and it follows that

$$
\sup _{B_{2-\delta}} w_{\varepsilon}-\frac{1}{2} \sup _{B_{2-\delta}}\left(\eta v_{\varepsilon}\right) \leq C\left\{\left\|f_{\varepsilon}\right\|_{L^{p}\left(B_{3}\right)}+\left(\int_{B_{2}}\left(v^{\varepsilon}\right)^{r}\right)^{1 / r}\right\} .
$$

This produces the desired bound by letting $\varepsilon \rightarrow 0$, since $v^{\varepsilon}, v_{\varepsilon} \rightarrow v$ uniformly in $B_{2}, B_{1} \subset B_{2-\delta_{\varepsilon}}$ for all $\varepsilon \leq \varepsilon_{0}$ and $f_{\varepsilon} \rightarrow f^{+}$in this case.

If $0<r<1$, we use the result just proved for $r=2$ to write

$$
\left(\int_{B_{2}} v^{2}\right)^{1 / 2}=\left(\int_{B_{2}} v^{2-r} v^{r}\right)^{1 / 2} \leq\left(\sup _{B_{2}} v\right)^{1-\frac{r}{2}}\left(\int_{B_{2}} v^{r}\right)^{1 / 2} .
$$

Then, by using (D.7) again with $p_{0}=\left(1-\frac{r}{2}\right)^{-1}=\frac{2}{2-r}, q_{0}=\frac{2}{r}>1$ and $\sigma=\frac{1}{2 C}\left(\int_{B_{2}} v^{r}\right)^{-1 / 2}$, we obtain

$$
\sup _{B_{2}} v \leq 2 C\left\{\left\|f^{+}\right\|_{L^{p}\left(B_{3}\right)}+\left(\int_{B_{2}} v^{r}\right)^{1 / r}\right\}
$$

On the other hand, if $r \geq p$, we just apply Holder's inequality on the right hand to derive the respective estimate with $r$.

Case 2. $f \in L^{p}\left(B_{3}\right)$ : Let $f_{k} \in L^{p}(\Omega) \cap C\left(B_{3}\right)$ such that $f_{k} \rightarrow f^{+}$in $L^{p}\left(B_{3}\right)$ and let $\psi_{k} \in W_{\text {loc }}^{2, p}\left(B_{3}\right) \cap C\left(\bar{B}_{3}\right)$ be the $L^{p}$-viscosity solution of

$$
-\mathcal{L}^{-}\left[\psi_{k}\right]=f_{k}-f^{+} \text {in } B_{3}, \quad \psi_{k}=0 \text { on } \partial B_{3}
$$

given, for instance, by theorem 4.6 in [38] (or by proposition 3.5). By ABP, $\left\|\psi_{k}\right\|_{L^{\infty}\left(B_{2}\right)} \rightarrow 0$. Now set $v_{k}:=v+\psi_{k}$. So, since $\psi_{k}$ is strong,

$$
-f^{+}-\nu(x) v \leq \mathcal{L}^{+}[v] \leq \mathcal{L}^{+}\left[v_{k}\right]-\mathcal{L}^{-}\left[\psi_{k}\right]=\mathcal{L}^{+}\left[v_{k}\right]+f_{k}-f^{+}
$$

i.e. $\mathcal{L}^{+}\left[v_{k}\right] \geq v(x) v_{k}+f_{k}(x)=\nu(x) v_{k}+\widetilde{f}_{k}(x)$ in $B_{3}$ in the $L^{p}$-viscosity sense, where $\tilde{f}_{k}:=f_{k}+\nu\left(v-v_{k}\right) \in C\left(B_{3}\right)$. Then case 1 yields the result, by

$$
\sup _{B_{2}} v_{k} \leq 2 C\left\{\left\|\widetilde{f}_{k}^{+}\right\|_{L^{p}\left(B_{3}\right)}+\left(\int_{B_{2}} v_{k}^{r}\right)^{1 / r}\right\}
$$

and letting $k \rightarrow+\infty$, since $\tilde{f}_{k}=f_{k}-\nu(x) \psi_{k} \rightarrow f^{+}$in $L^{p}\left(B_{3}\right)$.

Remark D.3 Notice that this version of LMP is sufficient for our purposes in section 5.2. We assumed $\nu(x)$ continuous in the proof above, but it is just a technicality when the functions $c$ and $h^{+}$in the problem $\left(P_{\lambda}\right)$ are bounded, with $\nu(x)=\Lambda_{2} c_{0}\left(1+m_{2} v_{2}\right)\left|\ln \left(1+m_{2} v_{2}\right)\right| / m_{2} v_{2}$ as in the proof of theorem 5.6. Indeed, say $c(x) \leq c_{0}, h^{+}(x) \leq h_{0}$, then $\nu v_{2} \leq|\nu|\left|v_{2}\right| \leq \widetilde{\nu}\left|v_{2}\right|$, where $\widetilde{\nu}$, defined as $\widetilde{\nu}(x)=\nu(x)$ if $v_{2} \neq 0$ and $\widetilde{\nu}(x)=\Lambda_{2} c_{0}$ if $v_{2}=0$, is a continuous function in $B_{2}^{+}$. Now observe that the proof above remains true if, instead of $\nu(x) u$, we have $\nu(x)|u|$, since $v=u^{+}$is still an $L^{p}$-viscosity subsolution of (2.6), and the rest of the proof carries on in the same way. 